\title{
Laser cavitation bubbles at objects: Merging numerical and experimental methods
}

\author{
Dissertation \\ zur Erlangung des mathematisch-naturwissenschaftlichen Doktorgrades \\ "Doktor rerum naturalium" \\ der Georg-August Universität Göttingen \\ im Promotionsprogramm ProPhys \\ der Georg-August University School of Science (GAUSS)
}

vorgelegt von

Max Koch

geboren in Konstanz, Deutschland

Göttingen, 2020 

Betreuungsausschuss:

Prof. Dr. Florentin Wörgötter

Drittes Physikalisches Institut Universität Göttingen

Dr. Robert Mettin

Drittes Physikalisches Institut

Universität Göttingen

Mitglieder der Prüfungskommission:

Referent:

Prof. Dr. Florentin Wörgötter Drittes Physikalisches Institut Universität Göttingen

Korreferent:

Prof. Dr. Ulrich Parlitz

MPI für Dynamik

und Selbstorganisation

Göttingen

weitere Mitglieder der Prüfungskommission:

Dr. Robert Mettin

Prof. Dr. Andreas Tilgner

Dr. Dieter Klopfenstein

Prof. Dr. Wolfram Kollatschny
Drittes Physikalisches Institut

Geophysikalisches Institut

Drittes Physikalisches Institut

Astrophysikalisches Institut
Universität Göttingen

Universität Göttingen

Universität Göttingen

Universität Göttingen

Tag der mündlichen Prüfung: 29.09.2020 
$\S 1$ of all laws of nature:

Respect nature.

$\S 1$ aller Naturgesetze:

Achte die Natur.

This thesis was written during Covid-19 time. 


\section{Contents}

1 Introduction 1

2 Theory 7

2.1 Laser generation of bubbles . . . . . . . . . . . . . . . . 7

2.2 Bubble dynamics . . . . . . . . . . . . . . . . 7

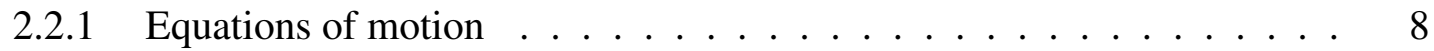

2.2 .2 Bubble energy . . . . . . . . . . . . . . . . 11

2.3 Erosion . . . . . . . . . . . . . . . . . . . . . 14

2.4 Numerics . . . . . . . . . . . . . . . . . . . . . . . . 15

2.4.1 Bubble simulation code . . . . . . . . . . . . . 15

2.4 .2 Lagrangian Inkmap . . . . . . . . . . . . . . . . . 22

2.4 .3 Boundary conditions . . . . . . . . . . . . . . 23

3 Setup, methods and preparations 25

3.1 Bubble numerics . . . . . . . . . . . . . . . . . 25

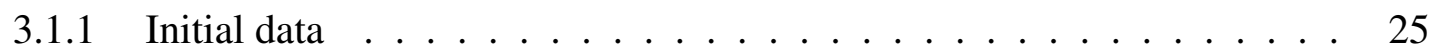

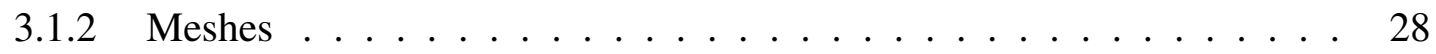

3.1 .3 Time stepping . . . . . . . . . . . . . . . . 33

3.1.4 Grid convergence study and best practice meshing . . . . . . . . 33

3.1.5 Correlate equilibrium radius and maximum radius . . . . . . . . . 34

3.2 Overlay method: ray-tracing to bring numerics and experiments to an overlay . 35

3.2 .1 Validation of the ray-tracing engine . . . . . . . . . . . 36

3.3 Experiments . . . . . . . . . . . . . . . . . . . . . 39

3.3.1 Setup 1: Large cuvette setup - kfps range . . . . . . . . . . . . . . 42

3.3.2 Setup 2: Small cuvette setup - close to Mfps range . . . . . . . . . . . . 44

3.3.3 Setup 3: Small cuvette setup - Mfps range . . . . . . . . . . . . 45

4 Bubble dynamics at the planar, solid boundary 47

4.1 Vortex generation and the Lagrangian Inkmap . . . . . . . . . . . . . 47

4.1.1 Results for different normalized bubble distances $D^{*} \ldots \ldots$. . . . . . 48

4.1.2 Discussion of the vortex results . . . . . . . . . . . . . 54

4.2 The jet-illusion bubble ..................... 61

5 The quest for the fast jet $\quad 65$

5.1 Bubble dynamics at $D^{*}=0 \ldots \ldots \ldots \ldots \ldots \ldots$

5.2 Full 3D simulation . . . . . . . . . . . . . . . . . . . 69

5.3 Quarter 3D simulation convergence study . . . . . . . . . . . . 70

5.4 Overlay with Mfps experiments . . . . . . . . . . . . . . . . 72 
6 Jet velocity, pressure on the solid boundary and maximum radius, dependent on $D^{*}$

6.1 Jet velocity . . . . . . . . . . . . . . . . . . . . . 76

6.2 Maximum radius . . . . . . . . . . . . . . . . . . 81

6.3 Pressure on the solid boundary $\ldots \ldots \ldots \ldots \ldots$. . . . . . . . . 82

7 Bubble dynamics close to a rigid cylinder - mushroom shape 86

7.1 Constant bubble energy . . . . . . . . . . . . . . . . . . 87

7.2 Different bubble energies . . . . . . . . . . . . . . . . . . . . 91

7.3 Simulations . . . . . . . . . . . . . . . . . . . 95

7.3.1 Convergence test numerics . . . . . . . . . . . . . . . . 996

7.3 .2 General dynamics . . . . . . . . . . . . . . . . . . . . . . . 97

7.3 .3 Several $D^{*} \ldots \ldots \ldots \ldots \ldots \ldots \ldots$

7.3.4 Two distinct simulations in full 3D . . . . . . . . . . . . . . 101

8 Conclusion 103

$\begin{array}{ll}\text { A Appendix - Fluid properties } & 110\end{array}$

B Appendix - Numerics 111

B.1 Grid convergence study and best practice meshing . . . . . . . . . . 111

B.1.1 Bubble in unbounded liquid - generating a reference solution . . . . 111

B.1.2 Words on saving calculation time . . . . . . . . . . . 115

B.1.3 Bubble close to a planar solid boundary . . . . . . . . . . . 116

B.2 Influence of the surface tension on the mushroom bubbles . . . . . . . . 121

B.3 Master-thesis, code and data availability . . . . . . . . . . . . . 122

B.3.1 Bubble case setup . . . . . . . . . . . . . . . . . . . . 123

B.4 Appendix - Benchmarking different machines . . . . . . . . . . . 123

$\begin{array}{ll}\text { C Experiments } & 127\end{array}$

C.1 Illumination . . . . . . . . . . . . . . . . . . . . . . . . . . . 127

C.1.1 Overdriven $100 \mathrm{~W}$ LED . . . . . . . . . . . . . . . . . . . 127

C.1.2 METTLE flash . . . . . . . . . . . . . . . . . . . . . . . . 134 


\section{Introduction}

The topic of this thesis is cavitation: the rupture of liquids due to tension or energy deposition. The most important liquid is

Water - the most peculiar liquid known. The simple triatomic molecule, the basis of life. The adult human has a water content of $65 \%-70 \%$ and water stabilises the helix structure of the DNA molecule (Franks 1972). The annual turnover of water on earth is estimated to $3.5 \cdot 10^{5} \mathrm{~km}^{3}=3.5 \cdot 10^{14}$ tons. Only $0.027 \%$ of the total water is fresh and immediately available. Most of the fresh water is locked in the Arctic and Antarctic ice caps. Ironically, in Franks (1972) a calculation has been posed that from an iceberg of size $(2700 \times 2700 \times 250) \mathrm{m}^{3}$ from the Antarctic Amery ice shelf $30 \%$ would arrive intact at Australia, worth 5.5 million \$, $10 \%$ of the cost of desalinated water back then. With the current climate change and the polar amplification effect, that phenomenon might occur naturally. The polar amplification effect refers to the phenomenon that the average local temperature rise due to climate change is highest at the polar regions (see e.g. Stuecker et al. (2018)).

The well known physical and chemical anomalies of water have significant impact, rarely fully realized. The large heat capacity of water ensures that the oceans, making up $97 \%$ of the earth's water, act as thermostats. This is also the reason why the energy imbalance of the climate change is buffered by $93 \%$ into the oceans (Cheng et al. 2019, for example). The density extremum at $4^{\circ}$ ensures life persistence by letting the earth's water only freeze on the surface. The compressibility $5.1 \cdot 10^{-10} \mathrm{~Pa}^{-1}$ reduces the ocean height by $40 \mathrm{~m}$ compared to incompressibility. The expansion upon freezing in combination with very high surface tension leads to penetrating smallest gaps in stones and cracking them, forming the basis of soil. Furthermore, water acts as an almost universal solvent. Almost every chemical compound is dissolved in water, at least to a slight extent. Very peculiar is the ability of the water molecule to form hydrogen bonds, meaning structured partnering of water molecules. These structures may maintain during thermal fluctuations at and below ambient temperatures (Chaplin 2019). This led to the term of water being a "structured" liquid (Franks 1972). The particle hydration shell of dissolved or dispersed particles can markedly be affected by the subtle altering of the water-water interactions due to presence of other molecules, substances, microorganisms or e.g. by heating, cooling, agitation, changes in $\mathrm{pH}$ and ionic strength (Franks 1972).

Cavitation - The topic of this thesis is rather connected to the ability of water (and also other liquids not investigated here) to mechanically erode even the hardest materials at the presence of cavitation bubbles. A cavitation bubble is understood as a millimeter or sub-millimeter sized bubble that undergoes explosive expansion and/ or rapid collapse dynamics due to short time, extreme events in temperature or pressure. Young (1999) states that Reynolds investigated cavitation bubbles in 1873 already. At the time, ship propellers were fast enough to produce cavitation clouds and dissipate energy into cavitation rather than into propulsion (see Fig. 1.1a,f). When the propeller cuts through the liquid, it produces low pressure regions, mainly at the tip of the blade and at the rotational axis, with values below vapour pressure ( $2337 \mathrm{~Pa}$ at $\left.20^{\circ}\right)$. This leads to explosive evaporation of the liquid parcels into vapour cavities, which then collapse again, and produce liquid jets towards the solid blade, potentially eroding it. Stronger 


\section{Introduction}

cavitation even occurs when the pressure falls below $0 \mathrm{~Pa}$, turning into tensile stress on the aforementioned hydrogen bonds. Impurities in the water then act as seeds of sudden rupture of the liquid. The cavity formed is then considered to only contain vacuum for a very short time until evaporation of water leads to filling with it vapour. Cavity formation in water due to static, low pressure is called hydrodynamic cavitation. Generating the low pressure acoustically creates acoustic cavitation. Cavitation by locally depositing energy by laser light is called optic cavitation and by detonation is called particle cavitation. This classification has been done by Lauterborn (1980) after the first cavitation conference in Göttingen in 1979. Furthermore, spark induced cavitation is known, where the bubble is induced by electrical discharge between two electrodes.

Apart from industrial occurences of cavitation, natural occurrences exist, too. The most pronounced example is that of the pistol shrimp (Synalpheus parneomeris) that has one claw much larger than the other, equipped with the ability to produce a cavitation bubble when clapping. $\mathrm{Au}$ and Banks (1998) measured the energy flux density of the clap shockwaves of 40 different shrimps. They found values between $127 \mathrm{~dB}$ and $135 \mathrm{~dB}$, corresponding to source energies of $17 \mu \mathrm{J}$ to $40 \mu \mathrm{J}$. Sperry et al. (1996) report on cavitation occuring in plants in the water conducting tissue (xylem) when it's partly dehydrated. When air enters the xylem, it gets compressed and may dissolve into neighbouring, water filled capillaries, where a cavitation bubble then is formed. Noblin et al. (2012) reported about a cavitation mechanism in fern when it ejects the spores.

Cavitation has found its way into a lot of different application fields such as, for instance, cleaning (Reuter et al. 2017b), food industry (Asaithambi et al. 2019), chemistry and biochemistry for enhancing reactions or waste water treatment (Gogate and Kabadi 2009) and medicine (Vogel and Venugopalan 2011; Brennen 2015; Pishchalnikov et al. 2019).

It is known since the beginning of discovery that cavitation is an erosive phenomenon. As stated later in Sec. 2.3, pressures of several hundred megapascal are necessary to erode alloys which e.g. ship propellers are made of. When exactly and how these pressures develop and which situations provoke higher pressures than others, is still to be clarified, although several decades of research have passed. On this way, cavitation has been found to include the topics of compressible fluid flow, non-linear acoustics, phase transition thermodynamics, non-linear optics and even nuclear physics. (see e.g. books and reviews: Leighton (1994), Brennen (1995), Young (1999), and Lauterborn and Kurz (2010)). Erosion happens on the sub-microsecond time scale and micrometer length scale, tempting experimental observation methods. The key point here is to understand the dynamics and processes of one single bubble. Usually, experiments comprise high-speed photography, laser induced or spark induced single bubbles and high intensity illumination for short camera shutter times (Lauterborn 1974; Lindau and Lauterborn 2003; Geisler 2004; Reuter and Mettin 2016; Supponen et al. 2016; Rosselló et al. 2018; Koch et al. 2020). The plasma formation due to laser light focusing into the liquid is called optical breakdown (Lauterborn and Kurz 2010; Köhler 2010).

Numerical methods using computational fluid dynamics (CFD) only roughly within the last decade evolved to simulate one single cavitation bubble with the sufficient inclusion of most relevant physics. Chahine (2014) and Chahine and Hsiao (2015) employ the linking of CFD and computational solid mechanics to find the stresses induced to solids by a single bubble. Pressures of adequate erosive values are found with inviscid fluids by applying sudden jumps of the atmospheric pressure of several magnitudes. Other authors (Johnsen and Colonius 2008; Johnsen and Colonius 2009; Müller et al. 2010; Ochiai et al. 2011; Lauer et al. 2012; Hsiao et al. 2014; Pishchalnikov et al. 2019) apply CFD for different bubble models and initial conditions of 


\section{Introduction}

(initially) spherical, cylindrical or planar bubbles, axisymmetric bubbles and three-dimensional bubbles in a constant external pressure field to shock-induced bubble collapse near boundaries as in lithotripsy.

The CFD solver of this thesis has a background of almost seven years of research (Han et al. 2015; Koch 2014; Lechner et al. 2015; Koch et al. 2016; Lechner et al. 2017; Lechner et al. 2018; Lauterborn et al. 2018; Lechner et al. 2019; Lechner et al. 2020; Koch et al. 2020). The solver intends to elucidate the fluid dynamics, especially that hidden to the experiment, of one single or few cavitation bubbles close to rigid objects. The idea behind the investigation of single cavitation bubbles in the laboratory and numerically is to clarify the dynamics of the smallest part of a cavitation erosion system. Fig. 1.1 sketches this idea.

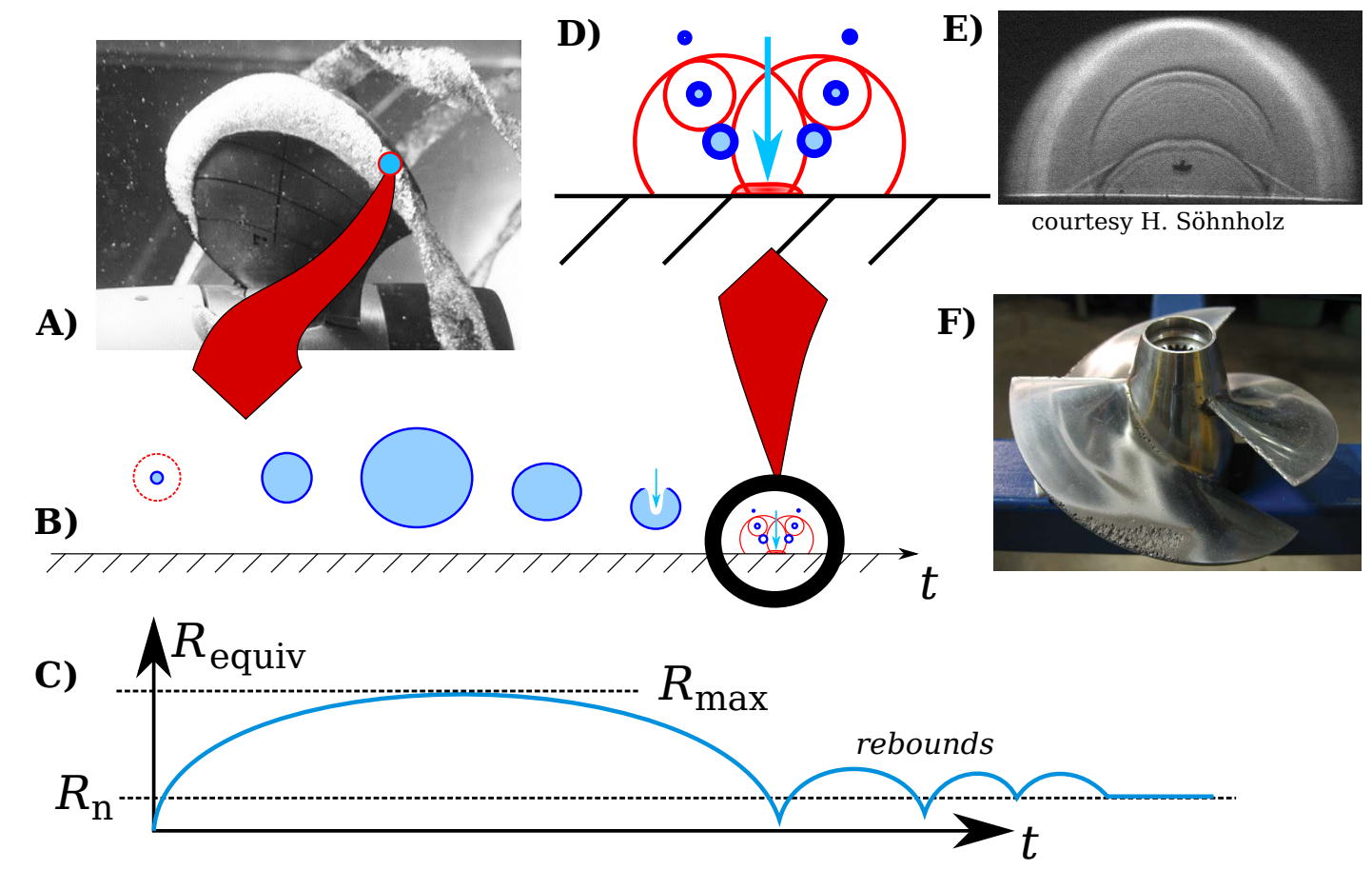

Fig. 1.1: Demonstration of the idea behind single bubble investigation: A) a rotating ship propeller with cavitation clouds at the blade tips (Wikimedia: Davidhv22 2011), where one bubble is extracted (B) that expands and collapses with shockwave emission (red) and liquid jet formation (blue arrow). The equivalent bubble radius $R_{\text {equiv. }}$ over time behaves like the curve drawn in C) where it expands to the maximum radius, collapses and undergoes several rebounds till it rests at equilibrium radius $R_{n}$. In the moment of jetting and minimum volume the bubble again emits shockwave patterns and the gas is split into tori fractions (D). An experimental image of the situation D) is shown in E) (Söhnholz 2016). After having studied single bubbles, the erosion damage on propeller (Wikimedia: Erik Axdahl (Liftarn) 2007) F) may be explainable.

(The following text part is similar but not identical to parts of Koch et al. (2020)):

Liquid jets and their experimental observation - A cavitation bubble in the vicinity of an object has a complicated two-phase, three-dimensional topology (see, e.g., Lindau and Lauterborn (2003)). Of special interest is the formation of fast liquid jets that appear in non spherically-symmetric environments (see e.g. for experimental photographs other than those of this thesis: Supponen et al. (2016) and Rosselló et al. (2018)). These jets are considered to be one of the reasons for erosion. They are formed in either of two ways: either by involution of 


\section{Introduction}

the bubble interface (Plesset and Chapman 1971; Lauterborn and Bolle 1975) due to restricted flow by the nearby object, or by impact of an annular inflow that then squeezes out the liquid in the respective orthogonal direction (Voinov and Voinov 1976; Lechner et al. 2019). After formation, the jet traverses the interior of the bubble and impacts onto the opposite bubble wall.

To follow this phenomenon it is necessary to have optical access to the interior of the bubble. Up to now, this is mostly done by diffuse illumination from the back (shadowgraphy) or multiple light sources to better show the topography of the deformed bubble surface. In shadowgraphy, smooth bubbles then appear black on a bright background with a bright center, where the light can pass undeflected (e.g., Lauterborn (1980), Reuter and Mettin (2016), and Rosselló et al. (2018)). The dark view of the rest of the bubble interior is due to the light being deflected off the surface of the bubble and not being able to reach the photographic film or the CCD chip of an electronic camera. An iconic, historic example of a laser generated bubble jet (Lauterborn 1980) is presented in Fig. 1.2a. The bubble is in its re-expansion phase (rebound) after the first collapse. The jet is due to the asymmetry introduced by a solid boundary below the bubble in the direction of the jet. Because only part of the interior of the bubble is optically accessible, the motion of the jet through the bubble can not be followed in its entirety. Lindau and Lauterborn (2003) could photograph the very formation of the jet by involution of the top of the bubble by applying special illumination and observation.

Overlay of numerical and experimental results - Regarding cavitation bubble dynamics, most publications focus either on numerical or on experimental results, and very few are known that compare experimental and numerical results directly (Lauterborn and Bolle 1975; Ohl and Ory 2000; Blake et al. 2015; Han et al. 2015; Koch et al. 2016; Pishchalnikov et al. 2019). This might be due to historical reasons, but with nowadays technological advancement it became possible to simulate the whole experiment.

Pursuing the goal to illuminate the whole volume of a small, air-filled sphere in water, a simple ray-tracing calculation based on Snell's law (code: Koch (2020)) would give the graphic in Fig. 1.2 b. Here, a case is considered, where the real experiment makes use of a long-distance microscope observing a tiny bubble. Thus it can be assumed that only parallel rays will enter an objective to the left of the bubble in the graphic. It is seen that the objective is hit by rays coming from a conical region behind (to the right) of the bubble. The cone has an aperture angle of about $90^{\circ}$. Therefore, in order to illuminate most of the bubble interior an illumination from behind the spherical bubble with the size of the base of that cone would be necessary. Still, the interior would be mapped towards the center region in the image seen by the microscope, leaving a thick dark outline at the bubble rim. However, this dark rim of the bubble might again be lightened by oblique incidence of light rays due to total reflection, such that the real interface position may become blurred or unclear (compare top and lowermost three rays of Fig. 1.2 b).

Analytical investigations of Snell's law for a circular interface have been used for a droplet (Kobel et al. 2009) and also for cylindrical or spherical glass vessels filled with sulfuric acid (Rosselló et al. 2016). In the latter, this method was combined with stereo camera recording and iterative triangulation to determine the position of a bubble inside the vessel. It is important to note that the previous analytic methods are useful only in cases with very simple geometries, and thus cannot be applied to a strongly aspherical bubble.

As a general rule, when the shape of the bubble under analysis becomes increasingly complex, it is almost impossible to reconstruct the shape by simple observation. Fig. $1.2 \mathrm{c}$ and Fig. $1.2 \mathrm{~d}$ are supposed to show the same bubble in experiment and in numerics, generated in direct vicinity to a solid surface, but it is evident that it is left to the eye of the observer whether the shapes are similar or not. The experimental bubble in Fig. $1.2 \mathrm{c}$ was illuminated from the 


\section{Introduction}

back, as well as from the right side to give it a more 3D appearance. As stated before, the background illumination is distorted strongly by refraction in the experimental image due to the interface of the bubble.

To determine the speed and shape of the liquid jets, a clear view into the interior of the bubble is required and more sophisticated illumination schemes must be implemented. In this thesis, as well as in Koch et al. (2020), the overlay method is presented, which makes use of the raytracing method to analyse complex scenarios. The optically relevant parts of the experiment are digitally reconstructed so that numerical results can be ray-traced and put to a similar image as gained in the respective real experiment.

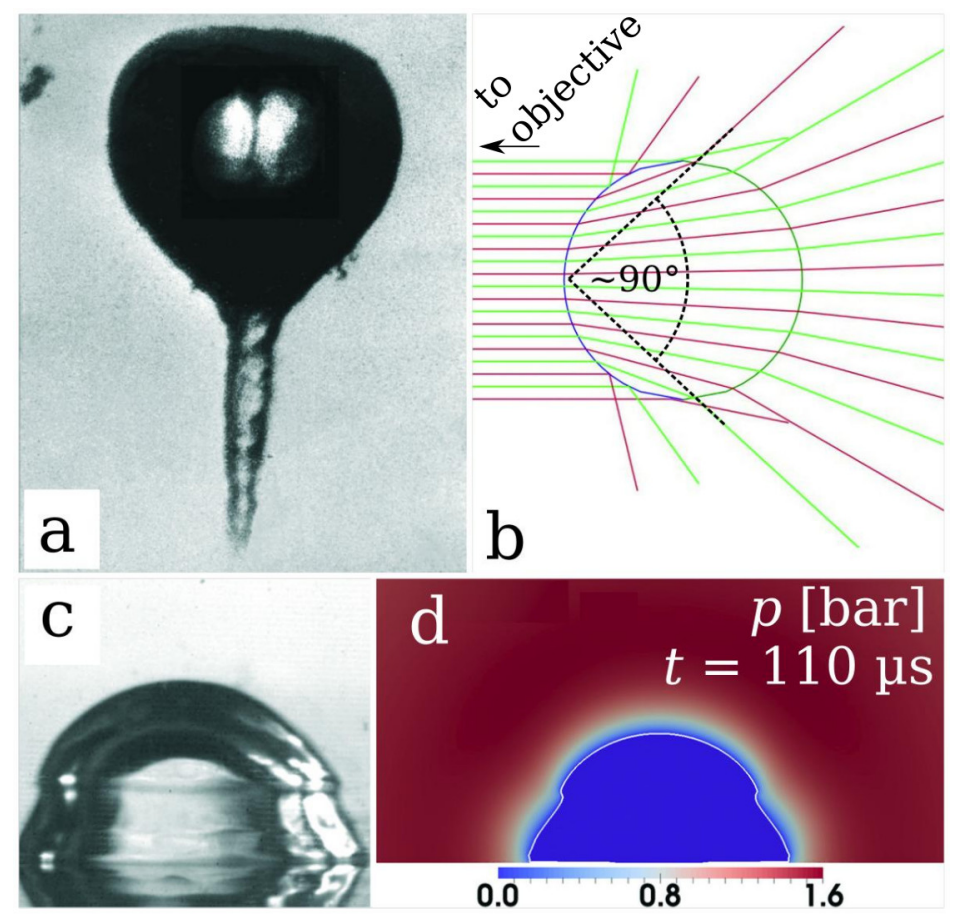

Fig. 1.2: a) Bubble with jet photographed in backlight (shadowgraphy). b) Applying Snell's law to a spherical air bubble in water for a bundle of parallel light rays. c,d) Standard comparison of an experimental, photographic image of a collapsing bubble in water (c) and a color coded visualization of the pressure in a corresponding numerical simulation as a cut through the bubble (d).

Recently, the ray-tracing method was used in the same sense as presented here, but for singlephase fluid dynamics: A Schlieren image from Large Eddy simulations was compared with experiments of a supersonic gas flow by Luthman et al. (2019). The authors implemented a ray-tracing engine themselves and found very good agreement of the resulting images between experiment and simulation for several flow features, e.g., jet-shock interaction and inferred velocity fields.

Ray-tracing of the two-phase flow of a single cavitation bubble was briefly introduced twenty years ago by Ohl and Ory (2000). Applying nowadays computational tools allow for investigations in much higher detail. Here blender is used, a free and open-source 3D animation tool with a python scripting interface, but the same technique can be also implemented by any optical ray-tracing software. Apart from the enhanced readability of scientific results, it can serve as an alternative validation method of any newly developed (two-phase) computational fluid dynamics simulation code by comparison with the experiment. Another possibility is to 
redesign experiments in order to optimize the visualization of specific expected phenomena.

Jet speed illusion and the fast jet - In this thesis, the overlay method is used to infer the shape of a strongly aspherical bubble close to a solid boundary and show that refraction leads to the optical illusion of a wrong liquid jet speed. The method furthermore aids to consolidate the arguments that the so called fast jet truly exists. The fast jet is predicted in Lechner et al. (2019) and Lechner et al. (2020) to reach a speed in the order of $1000 \mathrm{~m} / \mathrm{s}$ within 20 nanoseconds, yielding an astronomical acceleration one would not expect to meet in an ordinary lab. Since this type of jet only happens in a time window of less than one microsecond when the bubble is generated very close to, if not touching the solid boundary, and when it is already almost collapsed, it is no wonder that the jet has escaped observation for so long. It is still hard to fundamentally prove its existence by direct observation, yet indirect arguments are plentiful. To the thorough numerical investigations in axial symmetry in Lechner et al. (2020) in this thesis numerical results in full 3D, as well as experimental results are added and complemented with the overlay comparison.

Other publications showing the fast jet without explicitly paying higher attention to it are Benjamin and Ellis (1966), showing experimental results, and Pishchalnikov et al. (2019) showing numerical results with a completely different code.

This work is organized as follows: First the theoretical background of the bubble dynamics, erosion and numerical solver are explained in chapter 2. In chapter 3 the experimental and numerical arrangements are described. For the experiment three different setups were employed. One extra setup was used to validate the ray-tracing engine of blender by a static bubble attached to a syringe. Concerning the numerics, essential logical steps such as grid convergence studies or other validation investigations are put into the appendix in order to leave a clear story-line in the main body of the thesis. The appendix is understood as a rich compendium of resources needed to reproduce the results of this thesis. The links for the permanent storage of the resources are given there, too.

In chapter 4, 5 and 7 the results are reported. The vortices generated by a bubble close to a solid boundary are investigated in Ch. 4 and compared to the experimental data of Reuter and Mettin (2016) as a means to give a validation for the solver for long term fluid motions around a bubble. The aforementioned optical jet speed illusion is investigated here as well. The phenomenon of the fast jet is investigated in Ch. 5 with numerical, experimental and overlay methods. In Ch. 7 bubbles on top of a rigid cylinder of comparable radius are investigated both numerically and experimentally showing interesting shape dynamics reminding of mushrooms. 


\section{Theory}

In this chapter the relevant equations and relations are stated. If the detailed derivation can be found in Koch (2014) or in subsequent papers, only the result is stated here with a reference annotation.

\subsection{Laser generation of bubbles}

Following the tradition of my predecessors (Ohl 1998; Lindau 2001; Geisler 2004; Söhnholz 2016; Reuter and Mettin 2016), as well as the founders (Lauterborn 1974; Lauterborn 1980; Vogel 1987), the bubbles under investigation in this thesis are laser induced. This is considered non-invasive, to a certain extent, in contrast to spark induced bubbles where electrodes influence the dynamics. The deposition of energy is preferred, since the generation of a single bubble via tensile stress is very difficult to realize. The theoretical background of the nonlinear optical breakdown of the focused laser light in water is given in Köhler (2010). The absorption of photons in water is modeled with the model of semiconductors of a band gap of $\Delta E=6.5 \mathrm{eV}$. At high photon densities, an electron can absorb many photons instead of only one and the molecule may be ionized. The free electron then can absorb bremsstrahlung when hitting another molecule, thus inducing a cascade ionization and plasma growth. Vogel and Lauterborn (1988) measured the energy ratio of a laser pulse that is converted into shockwave generation from breakdown. With an Nd:YAG laser and their focusing optics, bubble formation started at $1 \mathrm{~mJ}$ laser pulse energy. Geisler (2004) calculated that an intensity of $2.8 \cdot 10^{17} \mathrm{~W} / \mathrm{m}^{2}=2.8 \cdot 10^{13} \mathrm{~W} / \mathrm{cm}^{2}$ is needed for breakdown for femtosecond pulses. Vogel et al. (2005) calculated the threshold spectrum for several pulse durations for the main wavelengths used for femto- and nanosecond lasers $(1064 \mathrm{~nm}, 800 \mathrm{~nm}$ and $532 \mathrm{~nm})$ and found threshold values of $I_{t h r} \approx 10^{12} \mathrm{~W} / \mathrm{cm}^{2}$ for pulse lengths $>10^{-11} \mathrm{~s}$ using a critical electron density of $\rho_{c r}=10^{21} / \mathrm{cm}^{3}$. This is the same order of magnitude confirmed by Köhler (2010).

\subsection{Bubble dynamics}

(This section is similar to parts in Koch et al. (2016). Details have been updated.)

The standard model for a cavitation bubble dominated by inertial forces is selected for the present investigations (Brennen 1995). It neglects mass and heat diffusion across the bubble interface as well as phase change effects and is valid for cavitation bubbles in water at normal ambient temperatures. Neglecting heat diffusion is justified taking into account the experiments in Söhnholz (2016), where no evidence of measurable temperature effects onto the liquid by the bubble could be found. Mass diffusion neglect is justified retroactively by showing that the bubble dynamics are well captured. Phase change effects are neglected owing to their complicated nature. Ishiyama et al. (2013) investigated the non-equilibrium kinetic boundary conditions at the bubble interface with molecular dynamics, intending to find the thermal accommodation 
coefficient, as well as evaporation and condensation coefficients for the interface boundary condition. Including these findings into a numerical solver for 3D bubble dynamics is a research project of its own.

The dynamics of the cavitation bubbles considered here is dominated by inertia and compressibility effects. Compared to the latter, heat diffusion effects are negligible. This has been found experimentally by Vogel and Lauterborn (1988) by measuring the energy of the shock wave radiated upon strong bubble collapse. More than $80 \%$ of the bubble energy may be radiated away upon collapse. Similarly, gas diffusion through the bubble wall can be neglected, as the time scale of diffusion is much larger than the time scale of the bubble oscillations considered here.

Evaporation and condensation on the other hand are fast processes with respect to bubble motion as considered here. This leads to a quick equilibration of the vapor pressure in the bubble, the saturation vapor pressure at the given temperature. In a cold liquid, i.e. a liquid far from its boiling point, this pressure is small with respect to the ambient pressure and therefore can be neglected to first approximation.

Altogether it can be stated that in the cases to be considered, thermodynamic effects play a minor role for the dynamics of the bubble as a whole. To a good approximation the bubble interior - the content of the bubble - can be modeled as a non-condensable gas of constant mass undergoing adiabatic changes of state.

The bubble exterior - the surrounding liquid - is taken to be (nonlinearly) compressible. Both, viscosity of the liquid and surface tension of the interface are included in the present investigations. Their effect on the bubble dynamics in a spherically symmetric collapse is marginal for the bubbles considered here. In the asymmetric collapse close to a solid wall they may have an influence on the dynamics and jet formation, as stated in Lechner et al. (2017). For distinct cases in this thesis, the difference will be shown. Also, viscosity of the liquid is needed to capture the boundary layer next to the solid wall. This is especially important for bubbles very close to the solid boundary, because it is the reason for the development of a fast jet of speed in the order of $1000 \mathrm{~m} / \mathrm{s}$.

The effect of gravity is small for the size and lifetime of the bubbles calculated here, so gravity is neglected.

\subsubsection{Equations of motion}

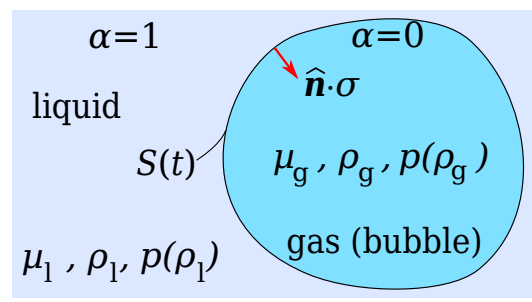

Fig. 2.1: Sketch of the bubble model.

The two fluids liquid and gas are considered to be separated by an interface of finite thickness. The equations of motion are formulated for a single fluid with a density field $\rho(\mathbf{x}, t)$, velocity field $\mathbf{U}(\mathbf{x}, t)$ and pressure field $p(\mathbf{x}, t)$ satisfying the continuity equation Eq. 2.4 and the momentum equation Eq. 2.3 given below. The standard values for the fluid properties are stated in the appendix in table A.1. 
In order to distinguish between liquid $(l)$ and gas $(g)$, volume fractions $\alpha_{i}(\mathbf{x}, t)$ with $i=l, g$ are introduced: $\alpha_{l}=1$ in the liquid phase and $\alpha_{l}=0$ in the gas phase, whereas $\alpha_{g}=1$ in the gas phase and $\alpha_{g}=0$ in the liquid phase. The position of the interface is then given implicitly by the transition of $\alpha_{l}$ from 1 to 0 . The volume fraction of the gas is related to $\alpha_{l}$ by

$$
\alpha_{g}(\mathbf{x}, t)=1-\alpha_{l}(\mathbf{x}, t)
$$

The overall density and viscosity fields can be written as

$$
\begin{aligned}
& \rho(\mathbf{x}, t)=\alpha_{l}(\mathbf{x}, t) \rho_{l}(\mathbf{x}, t)+\alpha_{g}(\mathbf{x}, t) \rho_{g}(\mathbf{x}, t) \\
& \mu(\mathbf{x}, t)=\alpha_{l}(\mathbf{x}, t) \mu_{l}+\alpha_{g}(\mathbf{x}, t) \mu_{g}
\end{aligned}
$$

with $\rho_{l}$ and $\rho_{g}$ the densities of liquid and gas, respectively. The dynamic viscosities $\mu_{l}, \mu_{g}$ are taken to be constant. The model is sketched in Fig. 2.1.

As phase transitions are neglected, there is no mass transfer between the fluids which is reflected in the continuity equations Eq. 2.5 for the individual fluids. Only two equations out of Eq. 2.4 and Eq. 2.5 are necessary, the third one is satisfied identically by virtue of the algebraic condition Eq. 2.1. The Navier-Stokes equation Eq. 2.3 and the continuity equations read

$$
\begin{aligned}
\frac{\partial(\rho \mathbf{U})}{\partial t}+\nabla \cdot(\rho \mathbf{U} \otimes \mathbf{U}) & =-\nabla p+\int_{S(t)} \sigma \kappa\left(\mathbf{x}^{\prime}\right) \hat{\mathbf{n}}\left(\mathbf{x}^{\prime}\right) \delta\left(\mathbf{x}-\mathbf{x}^{\prime}\right) \mathrm{d} S^{\prime}+\nabla \cdot \mathbb{T} \\
\frac{\partial \rho}{\partial t}+\nabla \cdot(\rho \mathbf{U}) & =0, \\
\frac{\partial\left(\alpha_{i} \rho_{i}\right)}{\partial t}+\nabla \cdot\left(\alpha_{i} \rho_{i} \mathbf{U}\right) & =0, \quad i=l, g
\end{aligned}
$$

$\nabla$ denotes the gradient, $\nabla \cdot$ is the divergence and $\otimes$ the tensorial product. The surface tension $\sigma$ is taken to be constant. The surface integral in Eq. 2.3 represents the surface force acting at the liquid/gas interface, see Tryggvason et al. (2001). $\kappa$ is twice the mean curvature of the interface and $\hat{\mathbf{n}}$ the unit normal to the interface, taken to point from the gas into the liquid here. $\delta\left(\mathbf{x}-\mathbf{x}^{\prime}\right)$ denotes the Dirac delta in three dimensions, with $\mathrm{x}^{\prime} \in S(t)$ a point on the interface and $\mathbf{x}$ the point at which the equation is evaluated.

$\mathbb{T}$ is the part of the viscous stress tensor $\mathbb{T}_{v}$ of a Newtonian fluid without bulk viscosity:

$$
\begin{aligned}
\mathbb{T}_{v} & :=\mu\left(\nabla \mathbf{U}+(\nabla \mathbf{U})^{T}-\frac{2}{3}(\nabla \cdot \mathbf{U}) \mathbb{I}\right) \\
\mathbb{T} & :=\mu\left(\nabla \mathbf{U}+(\nabla \mathbf{U})^{T}\right)
\end{aligned}
$$

with $\mathbb{I}$ the unit tensor. Mind that

$$
\begin{aligned}
(\nabla \mu) \cdot(\nabla \mathbf{U})^{T} & =(\nabla \mathbf{U}) \cdot(\nabla \mu) \\
\text { and } \frac{\partial}{\partial x^{j}} \frac{\partial u^{j}}{\partial x^{i}} & =\frac{\partial}{\partial x^{i}} \frac{\partial u^{j}}{\partial x^{j}}
\end{aligned}
$$

with exchanging the derivation order in the tensor notation of $\nabla \cdot(\nabla \mathbf{U})^{T}$. This leads to

$$
\nabla \cdot \mathbb{T}=\nabla \cdot(\mu \nabla \mathbf{U})+(\nabla \mathbf{U}) \cdot(\nabla \mu)+\mu \nabla(\nabla \cdot \mathbf{U})
$$


The complete $\nabla \cdot \mathbb{T}_{v}$ is derived in Koch (2014) and would read

$$
\nabla \cdot \mathbb{T}_{v}=\nabla \cdot(\mu \nabla \mathbf{U})+(\nabla \mathbf{U}) \cdot(\nabla \mu)+\frac{1}{3} \mu \nabla(\nabla \cdot \mathbf{U})-\frac{2}{3} \nabla(\mu)(\nabla \cdot \mathbf{U})
$$

Omitting the bulk viscosity is generally justified because i) the prefactor $2 / 3$ varies among different authors and ii) it is negligible in the problems under investigation even in strong compression cases. This was tested for a calculation with pressures up to $300 \mathrm{MPa}$ and by C. Lechner for the calculations in Lechner et al. (2020). Therefore the following analogy holds (with $\triangle$ the Laplace operator):

$$
\begin{aligned}
\nabla \cdot \mathbb{T}: & =\nabla \cdot(\mu \nabla \mathbf{U})+\underbrace{\nabla \mathbf{U} \cdot \nabla \mu}_{=0 \forall \mathbf{x} \notin S(t)}+\mu \nabla(\nabla \cdot \mathbf{U}) \\
\rightarrow & \nabla \cdot \mathbb{T}=\mu \triangle \mathbf{U}+\mu \nabla(\nabla \cdot \mathbf{U}) \quad \forall \mathbf{x} \notin S(t)
\end{aligned}
$$

Eqs. 2.3 to 2.5 are closed by the barotropic equations of state $(\operatorname{EoS}) \rho_{i}(p)$ given below. For negligible heat conduction and viscous dissipation energy then is automatically conserved.

EoS for the gas Upon strong (spherically symmetric) collapse, that brings the bubble size down to a few micrometer, the finite size of the gas molecules may play a role (Löfstedt et al. 1993). This is accounted for by including the co-volume in the equation of state, i.e. the gas in the bubble is described by the co-volume EoS (sometimes also called Noble-Abel EoS)

$$
\mathrm{R}_{\text {spec }} \vartheta=p\left(\frac{1}{\rho}-\frac{\beta}{\rho_{n}}\right)
$$

$\mathrm{R}_{\text {spec }}$ denotes the specific gas constant, $\vartheta$ the temperature and $\rho_{n}$ the equilibrium density of the bubble. $\beta$ is the co-volume de-dimensionalized with the molar volume at equilibrium. Common practice is to set $\beta=0$, though, for aspherically collapsing bubbles. The changes of state in the bubble are assumed to be adiabatic (an approximation often used for cavitation bubbles in liquids near normal conditions), therefore

$$
p\left(\frac{1}{\rho}-\frac{\beta}{\rho_{n}}\right)^{\gamma}=\mathrm{const},
$$

with $\gamma$ the ratio of specific heats. The polytropic exponent $\gamma=1.4$ is used for air or any two-atomic molecule gas.

EoS for the liquid For the liquid the Tait equation of state for water is used (see Li (1967), Fujikawa and Akamatsu (1980) and Cole (1948), p. 39 Eq. 2.30 for origin, as well as the book dedicated to the Tait equation itself: Aitken and Foulc (2019))

$$
p(\rho)=\left(p_{\infty}+B\right)\left(\frac{\rho}{\rho_{\infty}}\right)^{n_{T}}-B
$$

with $p_{\infty}$ the atmospheric pressure and $\rho_{\infty}$ the equilibrium density, the Tait exponent $n_{\mathrm{T}}=7.15$ and the Tait pressure $B=305 \mathrm{MPa}$. The Tait equation Eq. 2.16 models isentropic water based on data in the range of $0 \leq \vartheta \leq 45^{\circ}$ and 1 bar $\leq p \leq 1000$ bar. 
EoS derivatives $\psi_{i}$ The compressibility

$$
\psi_{i}=\frac{\mathrm{d} \rho_{i}}{\mathrm{~d} p}=\frac{1}{c_{i}^{2}},
$$

with $c$ being the speed of sound, of the respective $i$-th phase enters many equations. It has been found in Koch (2014) and Koch et al. (2016) that a special treatment has to be applied in order to stabilize the interface:

$$
\psi_{l, \text { corr }}=\alpha_{l} \psi_{l}+\alpha_{g} \psi_{\text {min }}, \quad \psi_{g, \text { corr }}=\alpha_{g} \psi_{g}+\alpha_{l} \psi_{\text {min }}, \quad \psi=\alpha_{l} \psi_{l, \text { corr }}+\alpha_{g} \psi_{g, \text { corr }}
$$

with $\psi_{\min }=10^{-16} \mathrm{~s}^{4} / \mathrm{m}^{2}$.

\subsubsection{Bubble energy}

As declared in the previous section, the bubble is assumed to be adiabatic, exchanging no heat or mass with the surrounding liquid. Wang (2016) then states that the local potential energy $E_{L P}$ of an infinitely slowly and adiabatically expanding and collapsing bubble is the sum of (i) the work done on the liquid by the gas pressure $p_{g}$ at the bubble interface and (ii) the power transmitted across a closed hyperplane around the bubble:

$$
E_{L P}=\underbrace{-\int_{V_{0}}^{V} p_{g} \mathrm{~d} V}_{(i)}+\underbrace{p_{\infty}\left(V-V_{0}\right)}_{(i i)} .
$$

Here, surface tension, vapour pressure and gravity are neglected, in contrast to Wang (2016). Using the adiabatic relation (Eq. 2.15 with $\beta=0$ )

$$
p V^{\gamma}=\text { const. }=p_{0} V_{0}^{\gamma}
$$

the term (i) transforms to

$$
\begin{aligned}
-\int_{V_{0}}^{V}\left(p_{0}\left(\frac{V_{0}}{V}\right)^{\gamma}\right) \mathrm{d} V & =-p_{0} V_{0}^{\gamma}\left[\frac{V^{1-\gamma}}{1-\gamma}\right]_{V_{0}}^{V}=-\frac{p_{0} V_{0}^{\gamma} V^{1-\gamma}-p_{0} V_{0}}{1-\gamma} \\
& =\frac{p_{0} V_{0}}{\gamma-1}\left(\left(\frac{V_{0}}{V}\right)^{\gamma-1}-1\right) .
\end{aligned}
$$

Accordingly, the total energy at $t=0$ of a bubble hypothetically compressed from its equilibrium volume $V_{n}$ to the volume $V=V_{0}$ and put into a liquid of zero velocity and constant ambient pressure $p_{\infty}$ is given by

$$
E_{\mathrm{tot}}=-\frac{p_{n} V_{n}^{\gamma} V_{0}^{1-\gamma}-p_{n} V_{n}}{1-\gamma}=\frac{p_{0} V_{0}-p_{n} V_{n}}{\gamma-1},
$$

where the term ii) of Eq. 2.19 is omitted because at $t=0$ no volume work has been performed yet $\left(V=V_{0}\right)$. Without energy dissipation by viscous or acoustic effects, we can calculate the theoretical maximum radius by setting $E_{\text {tot }}$ equal to the sum of the gas pressure work from the bubble equilibrium to maximum extension and the liquid volume work for the full expansion:

$$
\begin{gathered}
E_{\mathrm{tot}} \stackrel{!}{=}-\int_{V_{n}}^{V_{\max }} p_{g} \mathrm{~d} V+p_{\infty}\left(V_{\max }-V_{0}\right) \\
\frac{p_{n} V_{n}}{\gamma-1}\left(\frac{V_{n}}{V_{0}}\right)^{\gamma-1} \stackrel{!}{=} \frac{p_{n} V_{n}}{\gamma-1}\left(\frac{V_{n}}{V_{\max }}\right)^{\gamma-1}+p_{\infty}\left(V_{\max }-V_{0}\right)
\end{gathered}
$$


The asymmetry in the limits of the terms roots from the quasi-static assumption of the expansion process applied onto a non-equilibrium problem. If the bubble would expand quasi-statically, it would asymptotically approach the equilibrium volume. This can be verified by solving the simplified force balance equation (ordinary differential equation)

$$
m \ddot{R}=\left(p_{b}-p_{\infty}\right) \cdot S=p_{\infty}\left(\left(\frac{R_{n}}{R}\right)^{3 \gamma}-1\right) 4 \pi R^{2},
$$

with $S$ the bubble surface area and $p_{b}$ the homogeneous, adiabatic bubble pressure. The next step towards non-equilibrium is the Rayleigh-Plesset equation:

$$
R \ddot{R}+\frac{3}{2} \dot{R}=\frac{1}{\rho_{l}}\left(p_{b}-p_{\infty}\right) .
$$

For a bubble in unbounded liquid with equilibrium radius $R_{n}=184.1 \mu \mathrm{m}$, equilibrium pressure $p_{n}=p_{\infty}=101315 \mathrm{~Pa}$ and starting radius $R_{0}=20.063 \mu \mathrm{m}$ a theoretical maximum radius of $R_{\text {max,th }}=602.989 \mu \mathrm{m}$ is found with Eq. 2.23 or by solving the Eq. 2.25.

In the full CFD simulation of the same bubble a maximum radius of $R_{\max }=471.4 \mu \mathrm{m}$ is attained. With this value the ratio of emitted and dissipated energy to the total energy can be calculated:

$$
E_{\text {comp }, \text { visc }} / E_{\text {tot }}=\frac{E_{\text {tot }}-\frac{p_{n} V_{n}}{\gamma-1}\left(\left(\frac{V_{n}}{V\left(R_{\max }\right)}\right)^{\gamma-1}-1\right)-p_{\infty}\left(V\left(R_{\max }\right)-V_{0}\right)}{E_{\text {tot }}} .
$$

For the numerical bubble $54.58 \%$ is found. The three solutions are shown in Fig. 2.2.

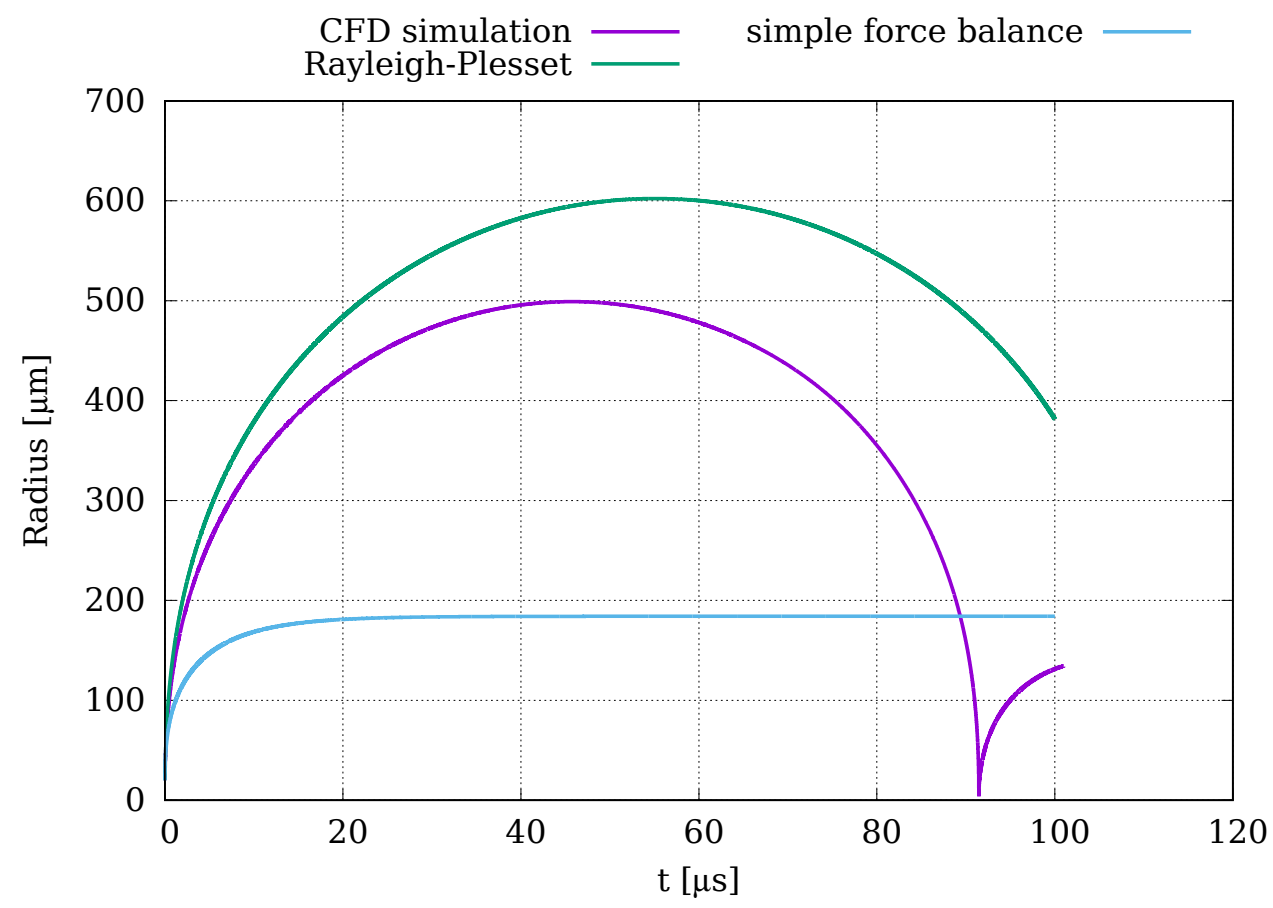

Fig. 2.2: Bubble radius over time curves, comparing the different concepts of energy conversion of the quasi-static, simple force balance Eq. 2.24, the Rayleigh-Plesset-Eq. 2.25 and the full CFD simulation (explained in the remainder body of the thesis). 
For the full CFD simulation all quantities can be directly extracted. The acoustical energy of the liquid is

$$
E_{\mathrm{ac}}(t)=\int_{\Omega} \alpha_{l}(\mathbf{x}, t) \frac{\left(p(\mathbf{x}, t)-p_{\infty}\right)^{2} \psi_{l}(\mathbf{x}, t)}{\rho_{l}(\mathbf{x}, t)} \mathrm{d} \mathbf{x}, \quad \psi_{l}=\frac{\mathrm{d} \rho_{l}}{\mathrm{~d} p}=\frac{1}{c_{l}(\mathbf{x}, t)^{2}},
$$

with $\Omega$ the volume of the computational domain, $\mathbf{x}$ the coordinate vector, $\psi_{l}$ the compressibility and $c_{l}$ the acoustic phase velocity in the liquid. The term of the gas pressure work of Eq. 2.19 according to Eq. 2.23 reads:

$$
E_{\text {pot,gas }}(t)=\int_{t\left(R=R_{n}\right)}^{t} \int_{\Omega} \alpha_{g}\left(\mathbf{x}, t^{\prime}\right) p\left(\mathbf{x}, t^{\prime}\right) \mathrm{d} \mathbf{x} \mathrm{d} t^{\prime}
$$

while the volume work term reads

$$
E_{\text {pot,liq }}(t)=p_{\infty}\left(\int_{\Omega} \alpha_{g}(\mathbf{x}, t) \mathrm{d} \mathbf{x}-\int_{\Omega} \alpha_{g}(\mathbf{x}, t=0) \mathrm{d} \mathbf{x}\right)
$$

and the kinetic energy reads

$$
E_{\text {kin }}(t)=\int_{\Omega} \alpha_{l}(\mathbf{x}, t) \frac{1}{2} \rho_{l}(\mathbf{x}, t)|\mathbf{U}(\mathbf{x}, t)|^{2} \mathrm{~d} \mathbf{x} .
$$

The quantities are plotted into Fig. 2.3. It is seen that the acoustic radiation of the bubble generation amounts to only about $25 \%$ which is then damped due to the computational grid. However, from the total amount of initial energy, the bubble turns $54 \%$ into volume work, yielding a loss of $21 \%$ somewhere. In contrast to the simulations in the following chapters, the bubble gas amount was kept constant here.

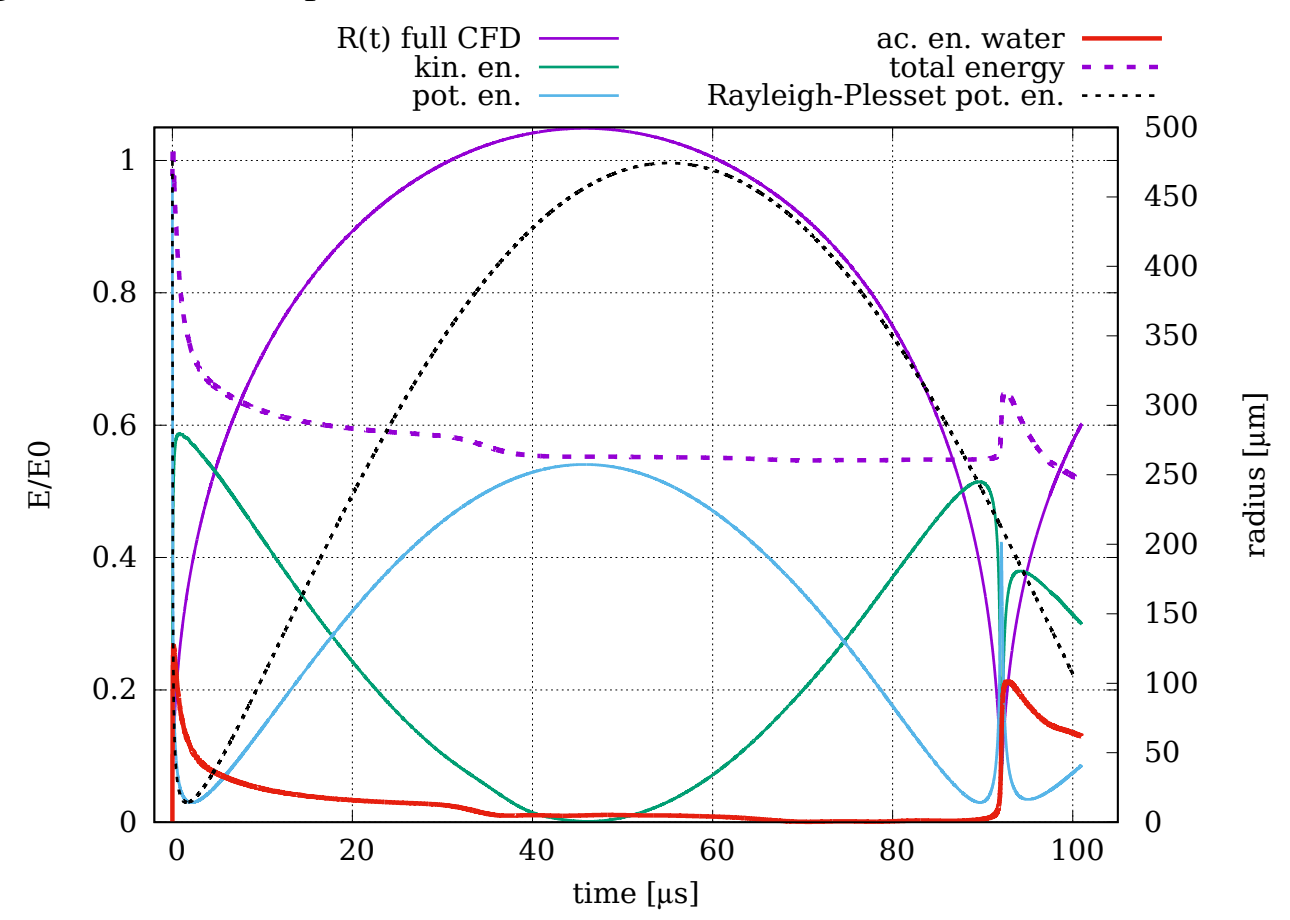

Fig. 2.3: Energies plotted for the full CFD simulation of a bubble with $R_{n}=184.1 \mu \mathrm{m}$, equilibrium pressure $p_{n}=p_{\infty}=101315 \mathrm{~Pa}$ and starting radius $R_{0}=20.063 \mu \mathrm{m}$. Viscosity is set to zero. Compared to the potential energy of the Rayleigh-Plesset solution.

This 21\%-issue has been discovered shortly before publishing and remains to be solved by the research community. 


\subsection{Erosion}

Metal alloys are and have to be flexible to some extent, otherwise products of those materials would immediately break under loading. Fig. 2.4 shows the schematic stress properties of most metal alloys. If the stress exerted on the material stays below the yield stress, it will fall back into original shape after stress release. Applying higher stresses leads to permanent deformation and exceeding the ultimate strength (German Zugfestigkeit) will break the probe. The yield strength is considered to be a value to judge cavitation erosion (Peters 2019, references therein). The standardized tensile test (European Committee for Standardization 2020) is used world wide to determine yield strength and ultimate strength. The strain rate at which the stress is measured is prescribed to maximum $0.0021 / \mathrm{s}$ in the norm, mimicking a quasi static experiment. At this condition, copper alloys used for ship propellers offer ultimate strengths of $450 \mathrm{MPa}$ (CC330G) to maximum $750 \mathrm{MPa}$ (CC333G) (Kupferinstitut 2010). The respective alloys are deformed by $0.2 \%$ at stresses of $160 \mathrm{MPa}(\mathrm{CC} 330 \mathrm{G})$ and $380 \mathrm{MPa}(\mathrm{CC} 333 \mathrm{G})$. As stated in Minuth-Hadi (2017), the stress increases by a factor of $\approx 1.1$ at a strain rate of $0.051 / \mathrm{s}$ and up to a factor of 1.8 at a strain rate of 400 1/s. This still does not fully explain the exceptionally high ultimate strength of $1000 \mathrm{MPa}$ Carnelli et al. (2011) measured with the nano-indentation method for the alloy C95800 applying a strain rate of $0.051 / \mathrm{s}$, intending to mimic cavitation forces. This alloy has an ultimate strength of $586 \mathrm{MPa}$ (Key to Metals AG 2020) in normal testing.
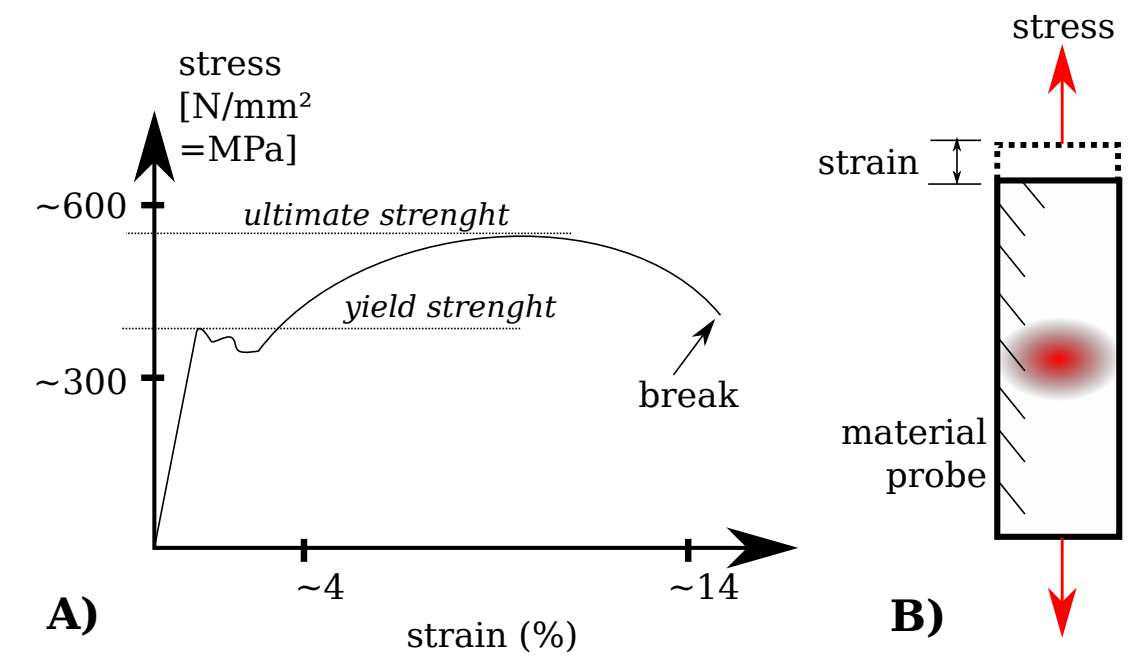

Fig. 2.4: Typical stress-strain curve of an alloy used e.g. for ship propellers (A) and the sketch (B) of the corresponding norm experiment using a probe. Exceeding the yield stress results into permanent deformation. If the ultimate strength is exceeded, the probe will will break.

Another phenomenon concerning alloys is the hardening effect, when the material is deformed. In this case both the stress needed for breaking is increased and the material loses flexibility. This effect attributes to the complexity of the erosion issue.

To conclude, for simplification, it can be said that cavitation induces damage to alloys when pressures of several hundred Megapascal are exerted to the solid surface.

Chahine and Hsiao (2015) predicted pressures of $1500 \mathrm{MPa}$ by a simulation of a single cavitation bubble with structure interaction. The authors employed the Euler-equations for the fluid, thus neglecting viscous damping, and used an instantaneous atmospheric pressure jump from 0.01 bar to 100 bar to induce bubble collapse.

In Lechner et al. (2017) we calculated maximum pressures of $\approx 95 \mathrm{MPa}$ for a bubble at nor- 
malized distance to the solid boundary of 1.42 in viscous, compressible water at 1 bar ambient pressure due to shockwave emission of the first collapse.

Erosion patterns of repeated single bubble measurements were investigated by Philipp and Lauterborn (1998) on pure aluminum (yield strength of $16 \mathrm{MPa}$ ) for normalized distances between 0.3 and 3 , as well as materials of yield strengths $235 \mathrm{MPa}$ and $483 \mathrm{MPa}$ for $\gamma_{d}=1.28$. It was found that for $\gamma_{d} \geq 2.2$ no damage to the aluminum probe happens, while for lower distances different damage patterns occur, indicating that erosive forces can mainly be attributed to shockwaves or direct bubble collapse at the solid boundary. Only for $\gamma_{d}<0.9$ a central smooth indent is observed.

In Sec. 6.3 the numerical simulations of this thesis will be investigated concerning the pressure at the solid boundary.

\subsection{Numerics}

The numerical solver and the discretization procedure is described in a very detailed manner in Koch (2014). The reader is furthermore referred to Koch et al. (2016), Lechner et al. (2017), Lauterborn et al. (2018), and Lechner et al. (2019) for more information and application. The key points are stated in this section as well as the relevant updates including the new local mass correction method. In Sec. 2.4.2 the numerical implementation of the Lagrangian inkmap (Reuter et al. 2017a) is described.

\subsubsection{Bubble simulation code}

The Navier-Stokes equation 2.3 and continuity equation 2.4 of the compressible, two-phase flow of a single, laser-generated cavitation bubble are discretized with the finite volume method (FVM) and the interface of the two fluids is treated with the volume of fluid (VoF) approach (Weller 2008). The solver is written within the OpenFOAM framework (Weller et al. 1998), precisely with the foam-extend-fork (Gschaider et al. 2017). The two compressible fluid phases are solved on the basis of the fields $\mathbf{U}, p, \alpha_{l}$ via the PISO algorithm (pressure implicit with splitting of operators). All other fields are derived from the main three, complemented by the equations of state 2.15 and 2.16. The flow charts in Figs. 2.5 and 2.6 visualize the steps of the solver. These are updated figures from the ones in Koch (2014). The solver is described top-down in the following paragraphs.

Time step initialization and time stepping - Before the outer correction loop starts (see Fig. 2.5), the time step is initialized and the control parameters are read in, so that they can be altered during run time. The Courant number for the global flow, interface flow and acoustic radiation is calculated, determining the time step size for adaptive time stepping. Not only the flow Courant number but also the acoustic Courant number and the flow Courant number of the bubble interface had to be considered. The flow Courant number is defined as the ratio of the flow speed $\mathbf{U}(\mathbf{x})$ at location $\mathbf{x}$ to the maximum resolvable flow speed by the spatial and temporal discretization $(\Delta x(\mathbf{x}), \Delta t)$ :

$$
\operatorname{Co}(\mathbf{x})=\frac{\Delta t}{\Delta x(\mathbf{x})} \cdot|\mathbf{U}|(\mathbf{x})=\Delta t \cdot C_{f}(\mathbf{x}) \cdot \frac{\phi(\mathbf{x}, i)}{\left|\mathbf{S}_{f}(\mathbf{x}, i)\right|}, \quad C_{f}(\mathbf{x})=\frac{\hat{\mathbf{n}}(\mathbf{x}, i) \cdot \delta(\mathbf{x})}{|\delta(\mathbf{x})|^{2}}
$$

where $\hat{\mathbf{n}}(\mathbf{x}, i)$ is the cell surface normal of cell at location $\mathbf{x}$ on its $i$-th cell face, $\delta(\mathbf{x})$ is the cell-to-cell distance vector, $\phi(\mathbf{x}, i)$ is the interpolation of $\mathbf{U}$ onto the $i$-th cell face multiplied 
with the surface vector $\mathbf{S}_{f}$ on that face. Usually, the maximum flow Courant number is set to

$$
\mathrm{Co}(\mathbf{x}) \leq \mathrm{Co}_{\max } \stackrel{!}{=} 0.2 \text {. }
$$

The acoustic Courant number is defined as:

$$
\mathbf{C o}_{\mathrm{Ac}}(\mathbf{x})=\Delta t C_{f} \cdot\left(\frac{\alpha_{l}(\mathbf{x}, i)}{\sqrt{\psi_{l}(\mathbf{x}, i)}}+\frac{\alpha_{g}(\mathbf{x}, i)}{\sqrt{\psi_{g}(\mathbf{x}, i)}}+\frac{\phi(\mathbf{x}, i)}{\left|\mathbf{S}_{f}(\mathbf{x}, i)\right|}\right)
$$

with $\alpha_{l}=1-\alpha_{g}$ the volume phase fraction and $\psi_{k} / \rho_{k}=1 /\left(\rho_{k} c_{k}^{2}\right)$ the compressibilities in the $k$-th phase with speed of sound $c_{k}$. Usually, the maximum acoustic Courant number is set to

$$
\mathrm{Co}_{\mathrm{Ac}}(\mathbf{x}) \leq\left.\mathrm{Co}_{\mathrm{Ac}, \max }\right|_{\forall t} \stackrel{!}{=} 8
$$

and for times where $R<r_{\text {crit }} R_{n}$ it is set to

$$
\mathrm{Co}_{\mathrm{Ac}}\left(\mathbf{x}, t\left(R<r_{\text {crit }} R_{n}\right)\right) \leq \mathrm{Co}_{\mathrm{Ac}, \max } \stackrel{!}{=} 1, \quad r_{\text {crit }}=0.3 .
$$

For explicit, fully coupled solvers, the maximum acoustic Courant number would be required to be less than 1 for all time.

The flow Courant number of the interface is defined as the Courant number of any flow in the interface region (not only the orthogonal one), but discretized with a volumetric method, skipping the cell-to-cell calculation:

$$
\begin{gathered}
\operatorname{Co}_{\alpha}(\mathbf{x})=\mathcal{P}\left(\alpha_{l}(\mathbf{x})-0.01\right) \mathcal{P}\left(0.99-\alpha_{l}(\mathbf{x})\right) \frac{1}{2} \frac{\sum_{i}|\phi(\mathbf{x}, i)|}{V_{\text {cell }}} \cdot \Delta t, \\
\mathcal{P}(x)=\left\{\begin{array}{ll}
0 & x<0 \\
1 & x \geq 0
\end{array},\right.
\end{gathered}
$$

with $V_{\text {cell }}$ the volume of the cell. Since $\phi$ is the integration of $\mathbf{U}$ over a surface, the unit is $\mathrm{m}^{3} / \mathrm{s}$. Now assuming a cubical cell with $\mathbf{U}$ of same direction and magnitude on opposite faces, the contribution to $\sum_{i}|\phi(\mathbf{x}, i)|$ would be $2 \mathbf{U} \hat{\mathbf{n}} S_{f}$, which is the reason for the half before the fraction. Assuming a cubical cell with $\mathbf{U}$ of opposite direction and same magnitude on opposite faces, the contribution to $\sum_{i} \phi(\mathbf{x}, i)$ would be zero, which is why the absolute value is taken in the sum. Eq. 2.36 has been implemented by C. Lechner (first author of Lechner et al. (2018)). Usually, the maximum Courant number for the interface is set to

$$
\mathrm{Co}_{\alpha}(\mathbf{x}) \leq \mathrm{Co}_{\alpha, \text { max }} \stackrel{!}{=}\left\{\begin{array}{ll}
0.08 & \mathrm{Co}_{\mathrm{Ac}, \max } \gg 100 \\
0.2 & \mathrm{Co}_{\mathrm{Ac}, \max } \lesssim 15
\end{array} .\right.
$$

There is also the restriction for the size of the time step for all times. The maxmimum size of the time step is usually set to $50 \mathrm{~ns}$.

Updating the equilibrium quantities, the model for condensation - Still before the outer correction loop, the equilibrium quantities of the bubble $R_{n}, p_{n}, \rho_{n}$ are updated. The bubble gas content is defined by the equilibrium radius $R_{n}$. In order to model both the strong first expansion and collapse, a very high gas content is chosen, that is linearly reduced in a specified time interval to one third approximately. The standard value for the initial equilibrium radius $R_{n, 1}$ is $184.1 \mu \mathrm{m}$, because this is the gas content needed for an expansion to $R_{\max }=495 \mu \mathrm{m}$ in 
unbounded liquid, starting at $R_{\text {init }}=20 \mu \mathrm{m}$. Usually this equilibrium radius is linearly reduced to $R_{n, 2}=64 \mu \mathrm{m}$ in the interval $60 \mu \mathrm{s}$ to $75 \mu \mathrm{s}$. This directly influences the mass correction algorithm explained later, thus reducing the density of the bubble. If $R_{n}$ is changed, $p_{n}$ and $\rho_{n}$ that enter the equation of state 2.15 have to be changed accordingly:

$$
\begin{aligned}
& R_{n}=R_{n}(t)= \begin{cases}R_{n, 1} & t<t_{a} \\
\left(t-t_{a}\right) \cdot\left(R_{n, 2}-R_{n, 1}\right) /\left(t_{b}-t_{a}\right)+R_{n, 1} & t_{a} \leq t<t_{b} \\
R_{n, 2} & t>t_{b}\end{cases} \\
& p_{n}=p_{n}(t)=p_{\infty}+2 \frac{\sigma}{R_{n}(t)}, \\
& \rho_{n}=p_{n}(t) \frac{1-\beta}{\mathrm{R}_{\mathrm{spec}} \vartheta_{0}}
\end{aligned}
$$

with $\mathrm{R}_{\text {spec }}$ the specific gas constant, $\vartheta_{0}$ the ambient temperature and $\beta$ the de-dimensionalized co-volume of the gas.

A transport equation for the volume fraction field $\alpha_{l}$ - is obtained when formulating the continuity Eq. 2.5 for the liquid phase in terms of the pressure:

$$
\frac{\partial \alpha_{l}}{\partial t}+\nabla \cdot\left(\alpha_{l} \mathbf{U}+\alpha_{l} \alpha_{g} \mathbf{U}_{i c}\right)=\alpha_{l} \alpha_{g} \frac{\mathrm{d} g}{\mathrm{~d} t}+\alpha_{l} \nabla \cdot \mathbf{U}
$$

where $\alpha_{l} \alpha_{g} \mathbf{U}_{i c}$ is an artificial interface compression term included to counteract numerical diffusion of the interface, acting only on the interface. Details for this term are stated in Koch (2014), equation 3.10. $\mathrm{d} g / \mathrm{d} t$ is defined as

$$
\frac{\mathrm{d} g}{\mathrm{~d} t}= \begin{cases}\left(\frac{\psi_{2}}{\rho_{2}}-\frac{\psi_{1}}{\rho_{1}}\right) & \frac{\mathrm{d} p}{\mathrm{~d} t} \quad \\ 0 & \text { else }: 0.001 \leq \alpha_{l}(\mathbf{x}) \leq 0.999\end{cases}
$$

with $\psi_{k} / \rho_{k}$ the compressibilities according to Eqs. 2.17 and 2.18. The case differentiation has been introduced for stability reasons. $\mathrm{d} p / \mathrm{d} t$ was calculated in the last time step and thus will be explained further downwards (Eq. 2.42).

The velocity matrix - firstly comprises only the lefhand side of Eq. 2.3 and $\nabla \cdot \mathbb{T}$ :

$$
\frac{\partial(\rho \mathbf{U})}{\partial t}+\nabla \cdot(\rho \mathbf{U} \otimes \mathbf{U})-\nabla \cdot \mathbb{T}=0
$$

If the predictor step is performed, too, then this equation is solved once with the source terms of the Navier-Stokes Eq. 2.3 on the right hand side by using old values for the pressure field.

PISO loop - In the PISO-loop the most complex part of the solver is concentrated, since the solver is pressure based. First, the compressible part of the pressure equation is set up. The compressible part refers to $\mathrm{d} p / \mathrm{d} t$ :

$$
\frac{\mathrm{d} p}{\mathrm{~d} t}=\frac{\partial p}{\partial t}+(\mathbf{U} \nabla) p=\frac{\partial p}{\partial t}+\nabla \cdot(p \mathbf{U})+p \nabla \cdot \mathbf{U}
$$

When discretizing the Eq. 2.41, a matrix $\mathcal{M}$ is formed for the equation system

$$
\mathcal{M} \cdot \mathbf{U}(\mathbf{x})=0
$$


This matrix can be expressed in terms of diagonal entries and off-diagonal entries. This is the same as saying that it can be expressed as the sum of the contribution by terms $\mathcal{A}_{p}$ from the same cell and terms from the neighbouring or related $\mathcal{A}_{r}$ cells:

$$
\mathcal{A}_{p} \mathbf{U}_{p}+\sum_{r} \mathcal{A}_{r} \mathbf{U}_{r}=0
$$

Temporarily, the field $\mathbf{U}_{p}$ is stored: (in the code called phiU):

$$
\mathbf{U}_{p} \equiv-\mathcal{A}_{p}^{-1} \sum_{r} \mathcal{A}_{r} \mathbf{U}_{r}
$$

where in OpenFOAM the so called $\mathcal{H}$-operator is

$$
\mathcal{H}=-\sum_{r} \mathcal{A}_{r} \mathbf{U}_{r}
$$

at least for this solver. Another field $\mathbf{U}_{p, \text { phi }}$ field is stored (in the code called $p h i$ ):

$$
\mathbf{U}_{p, \text { phi }} \equiv \mathbf{U}_{p}+\mathcal{A}_{p}^{-1} \mathbf{f}_{\sigma} .
$$

$\mathbf{f}_{\sigma}$ refers to the surface tension force term in Eq. 2.3, which reads (Koch 2014; Brackbill et al. 1992):

$$
\mathbf{f}_{\sigma}=-\sigma \nabla \cdot\left(\frac{\nabla \alpha_{l}}{\left|\nabla \alpha_{l}\right|}\right) \nabla \alpha_{l}
$$

PISO loop - Non-orthogonal correction loop - The incompressible part $\left(p_{\text {incomp }}\right)$ of the pressure equation with its matrix representation $\mathcal{P}_{\text {incomp }}$ is set up within the non-orthogonal loop, using the $\mathrm{U}_{p, \text { phi }}$ from Eq. 2.46:

$$
p_{\text {incomp }} \equiv \nabla \cdot \mathbf{U}_{p, \text { phi }}-\nabla \cdot\left(\mathcal{A}_{p}^{-1} \nabla p\right) .
$$

The equation now being solved iteratively is

$$
\left(\alpha_{l} \frac{\psi_{l}}{\rho_{l}}+\alpha_{g} \frac{\psi_{g}}{\rho_{g}}\right) \frac{\mathrm{d} p}{\mathrm{~d} t}+\mathcal{P}_{\text {incomp }}=0
$$

In the last iteration step the quantity $\mathrm{d} g / \mathrm{d} t$ is calculated (Eq. 2.40) and the velocity $\mathbf{U}$ is updated as

$$
\mathbf{U} \equiv \mathbf{U}_{p, \text { phi }}+\text { flux }\left(\mathcal{P}_{\text {incomp }}\right)
$$

where it remains obscure how the flux of the matrix $\mathcal{P}_{\text {incomp }}$ is computed and converted into a velocity. The respective code is far below the top level of OpenFOAM programming.

In the above description of the algorithm the steps are omitted where the velocity fields are transformed from midpoint to surface representation or the other way round. 


\section{Theory}

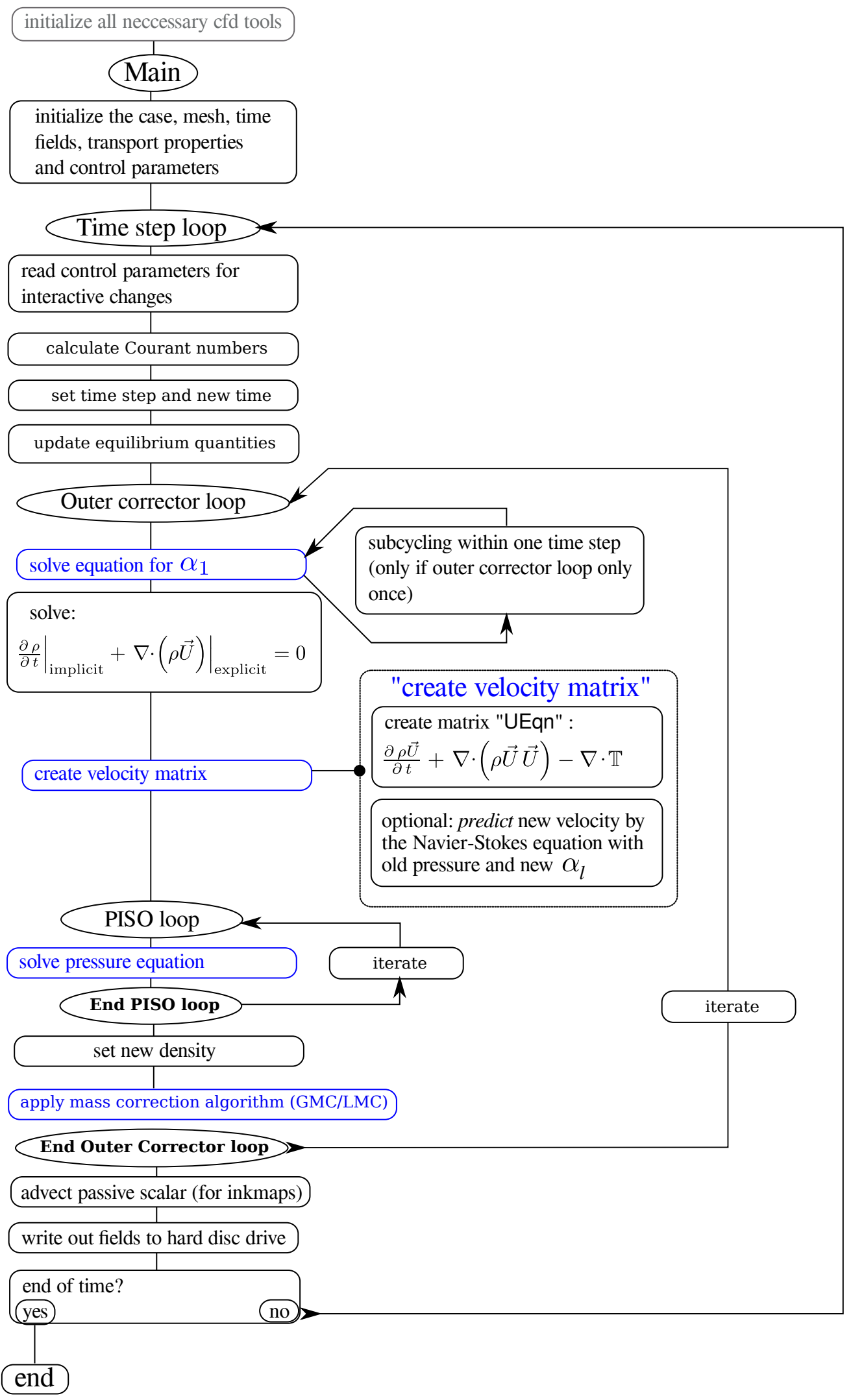

Fig. 2.5: Flow chart of the top level of the CFD solver. The blue boxes are explained in more detail elsewhere. The PISO loop flow chart is given in Fig. 2.6. 


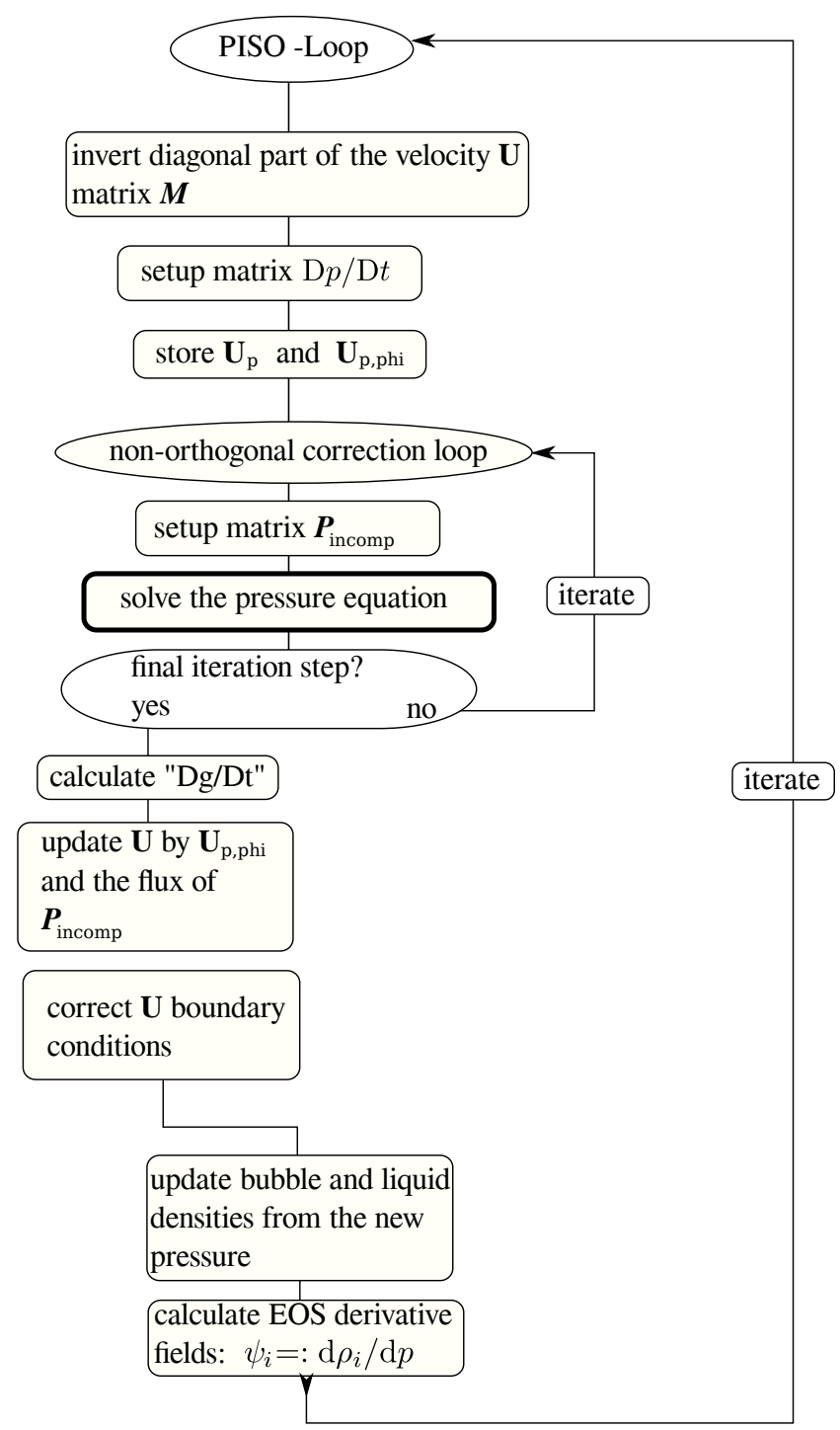

Fig. 2.6: Flow chart of the PISO loop of the CFD solver. The steps are explained in the text.

Mass conservation correction: Global versus local - In Koch (2014) and Koch et al. (2016) it was found that there is a huge error in the mass conservation of the gas phase (see figure 7 in the latter publication), when the bubble pressure increases. This was counteracted by a simple means, namely to just reset the gas density in every cell each time step by the factor of mass loss.

$$
\rho_{g, c o r r}(\mathbf{x}, t)=\frac{\int_{\Omega} \rho_{g}(\mathbf{x}, t=0) \mathrm{d} \mathbf{x}}{\int_{\Omega} \rho_{g}(\mathbf{x}, t) \mathrm{d} \mathbf{x}} \rho_{g}(\mathbf{x}, t),
$$

with $\Omega$ the volume of the computational domain. This method is called global mass correction (GMC) because this is done for the whole gas phase of the computation. It was shown to work very well in Koch et al. (2016). This method was applied in all the subsequent publications Koch (2014), Koch et al. (2016), Lechner et al. (2017), Lauterborn et al. (2018), and Lechner et al. (2020).

The main drawback of this method comes into play when the bubble is split geometrically from one connected domain to multiple domains. Because the error in mass is related to the bubble pressure, gas domains of high pressure can cause other gas domains of equilibrium 
pressure to lose mass and thus collapse again. Therefore, for multiple gas domains, the GMC is expected to yield wrong results.

The research on finding a formulation of a local mass correction (LMC) took quite long. In the end, C. Lechner was the first who found a stably working algorithm with a long term mass error of less than $1 \%$. It has not been reported since then, even though all results presented in this thesis are produced with the LMC. Therefore, it will be reported here for the first time, though the method finding credits are owned by C. Lechner:

After the PISO loop, an extra continuity equation for the gas phase density $\alpha_{g} \rho_{g}$ is solved with the following algorithm. First, a third phase parameter field $\widetilde{\alpha}_{g}$ aiding for temporary storage, is updated in the following way:

$$
\widetilde{\alpha}_{g}=\left\{\begin{array}{ll}
\alpha_{g} & \forall \mathbf{x}: \alpha_{g}>0.001 \\
0.001 & \text { else }
\end{array} .\right.
$$

This accounts for boundedness when the matrix is inverted, preventing dividing by zero. My contribution to the method was to find out that the field $\widetilde{\alpha}_{g}$ necessarily has to be declared before the time iteration routine as a full IOobject volScalarField, not only as a volScalarField. Now the implicit extra continuity equation can be solved with $\widetilde{\alpha}_{g}$ :

$$
\frac{\partial \widetilde{\alpha}_{g} \rho_{g}}{\partial t}+\nabla \cdot\left(\widetilde{\alpha}_{g} \rho_{g} \mathbf{U}\right)=0
$$

When the simulation time is in the time interval of the $R_{n}$-reduction, then after solving Eq. 2.53, a GMC step (Eq. 2.51) is performed with the new $R_{n}(t)$ (Eq. 2.38). To be safe for stability, the $\rho_{g}(\mathbf{x})$ field is limited to the minimum density corresponding to the minimum gas pressure, that is user-specified to a value usually of $2.5 \mathrm{~Pa}$. When correcting $\rho_{g}$, also the gas pressure has to be updated by the Eq. 2.15. The corrected pressure field afterwards reads:

$$
p_{\text {corr }}(\mathbf{x})=\alpha_{g} p_{g, \text { corr }}+\alpha_{l} p \text {. }
$$

When correcting $p$, also the liquid density has to be corrected again by Eq. 2.16. Afterwards the mass fluxes which are called rhoPhi, rho_lPhi and rho_gPhi are updated and finally the $\psi_{k}$-fields are re-evaluated, too (Eq. 2.17).

Validation of the LMC - The local mass correction method is validated indirectly by the following arguments:

- C. Lechner kept running the simulations with the GMC only. The results of both codes match since the invention of the LMC in early 2017.

- The grid convergence studies in the appendix Sec. B.1.1 shown in Fig. B.2 are tested with the GMC, too, yielding the same results.

- The striking results of Ch. 5 are shown to match concerning the results from C. Lechner and the ones obtained with the code for this thesis using the LMC.

- The LMC results of this thesis match with the experiments shown. 


\subsubsection{Lagrangian Inkmap}

Reuter et al. (2017a) measured the flow field in the cross section through a bubble close to a solid boundary. They invented the method they called Lagrangian inkmap, where the flow field is interpolated and numerically filled with a lot of particles that are then advected with a RungeKutta scheme. The particles are colored, so that the time integrated movement of the liquid can be made visible, just as if a layer of ink was put above the solid boundary in the experiment. This way they found that the bubble creates vortices that either float orthogonally away from the solid boundary, or spread alongside it. These findings are reproduced by CFD simulation in this thesis, Sec. 4.1. This can be considered a validation of the code for long term phenomena, since the vortices develop only in the millisecond time scale when the bubble has already vanished.

In order to produce a numerical inkmap, the Lagrangian approach has to be formulated in an Eulerian way. The field $\Gamma(\mathbf{x}, t)$ that has a color gradient in the $y$-direction (orthogonal to the solid boundary) while being constant in the other

$$
\Gamma(\mathbf{x}, t)= \begin{cases}\frac{\mathbf{x e}_{y}}{1.5 R_{\max }} & \forall \mathbf{x e}_{y}<1.5 R_{\max } \\ 1 & \text { else }\end{cases}
$$

is introduced. The origin $\mathbf{x}=(0,0,0)$ is in the center of the bubble at time zero. The field is advected by the incompressible continuity equation formulation:

$$
\frac{\partial \Gamma}{\partial t}+\nabla \cdot(\Gamma \mathbf{U})-(\nabla \cdot \mathbf{U}) \Gamma=0
$$

The compressible formulation would result into color compressibility and thus the limits of $\Gamma$ would not be maintained and the color of the Lagrangian particle would change. In order to validate the approach of Eq. 2.55, a post-processing utility was written which uses the velocity field of any foam-extend simulation to advect a number of Lagrangian color particles with an Euler step, just as was done in Reuter et al. (2017a) for the experiments. The average color per cell is mapped onto the grid and the result is compared to solving Eq. 2.55. The standard tutorial case of foam-extend called depthCharge was taken for testing because of the low computation times (sketch in Fig. 2.7). Fig. 2.8 shows the comparison result.

2D tutorial case

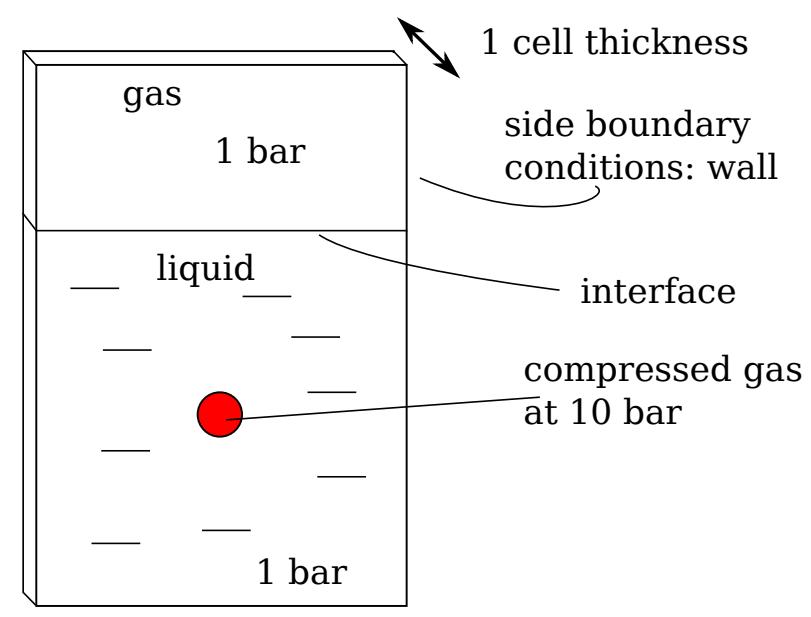

Fig. 2.7: Sketch of the standard OpenFOAM 2D depthCharge tutorial case used for the inkmap validation. 

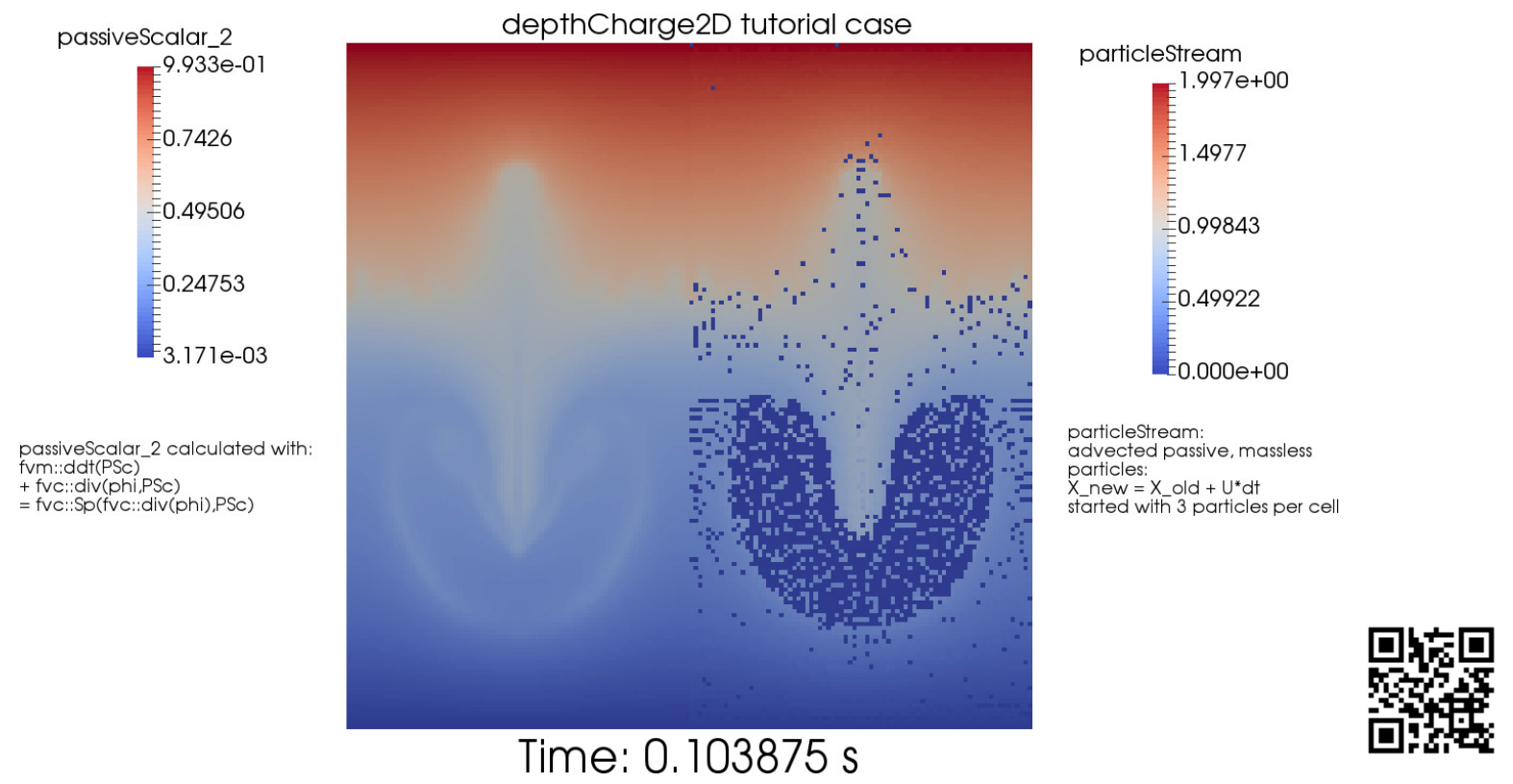

Fig. 2.8: Passive scalar advection (left) using Eq. 2.55 and Lagrangian color particles (right). Solving the depthCharge tutorial case. Movie online via QR-code or: https://doi.org/10.25625/RBZGLA

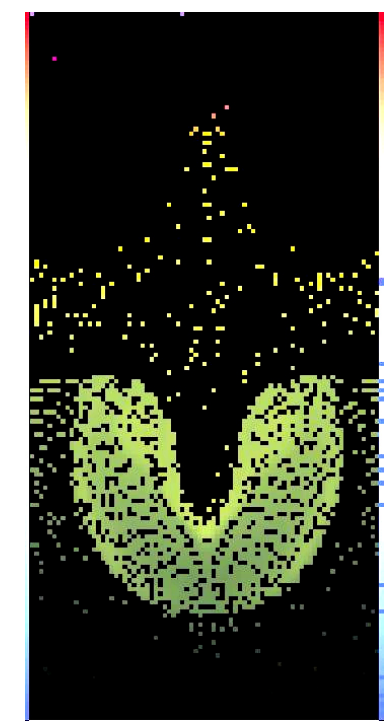

Fig. 2.9: Standard deviation of left and right frame of Fig. 2.8 showing clearly only the cells which are not filled with Lagrangian color particles.

\subsubsection{Boundary conditions}

The following set of boundary conditions is applied.

solid boundary:

$$
\nabla p=0,|\mathbf{U}|=0, \alpha_{l}=1 .
$$

$\alpha_{l}=1$ makes sure, the bubble will not touch the solid boundary, even for under-sampling the boundary layer. The same results are obtained with a boundary condition of zero gradient for 
the volume fraction for higher resolution at the solid boundary (Lauterborn et al. 2018).

Geometrical:

The OpenFOAM inherent wedge boundary condition is applied for the boundaries facing the angular direction for calculations in axial symmetry, to convert a 3D mesh of 1 cell thickness into an axisymmetric one. For the quarter $3 \mathrm{D}$ case in $\mathrm{Ch} .5$ the cyclic boundary condition is applied for the angular directions.

Outer boundary far from the bubble:

The waveTransmissive-boundary condition is applied to the outer boundary of the computational domain for the pressure $p$. The purpose is to approximate a non-reflective boundary with a relaxation to the value of $p_{\infty}$. The implementation seems to be done in the source code named waveTransmissive, advectiveFvPatch and mixedFvPatch.

$$
\begin{aligned}
w & =\max \left(\mathbf{U} \cdot \mathbf{n}_{b}+\sqrt{1 / \psi}, 0\right) \\
k_{1} & =\frac{w \cdot \Delta t}{\operatorname{IInf}}, \quad k_{2}=\frac{w \cdot \Delta t}{\delta(\mathbf{x})} \\
r_{1} & =\frac{p+k_{1} \cdot p_{\infty}}{1+k_{1}}, \quad r_{2}=\frac{\frac{3}{2}+k_{1}}{\frac{3}{2}+k_{1}+k_{2}} \\
p_{\text {new }} & =r_{1} \cdot r_{2}+\left(1-r_{2}\right) \cdot p
\end{aligned}
$$

where $w$ is understood as the wave speed which is set to 0 for "incoming waves" - at least in an incompressible case -, $k$ as a normalized wave-traveling distance with user-input parameter lInf, $k_{2}$ as the ratio of the wave-traveling distance to the grid spacing, $r_{1}$ as the so-called refValue and $r_{2}$ the so-called valueFraction. The vector $\mathbf{n}_{b}$ is understood as the normal vector at the boundary pointing out of the computational domain. The speed of sound is $c=\sqrt{1 / \psi}$. The cell-to-cell distance $\delta(\mathbf{x})$ has been introduced in Eq. 2.31. This is how the boundary condition is understood here. In the official source code the following explanation is given:

This boundary condition provides a wave transmissive outflow condition, based on solving

$$
\frac{\mathrm{d}(w \cdot p)}{\mathrm{d} t}
$$

at the boundary[.] $w$ is the wave velocity and $[p]$ is the field to which this boundary condition is applied.

For the velocity the pressureInletOutletVelocity is applied at the outer boundary. In the official OpenFOAM-API guide (OpenCFD-team and Nagy 2017) the boundary condition is described to adjust the velocity in the following way:

Flow out of the domain: assigns a zero gradient condition

Flow into the domain: assigns a velocity based on the flux in the patch-normal direction 


\section{Setup, methods and preparations}

The numerical methods of simulation and ray-tracing, as well as the experimental methods are described in this chapter.

\subsection{Bubble numerics}

In this section the way a simulation is set up is described. Most of the code is available online, the repositories of the code and scripts are given in Appendix B.3.

\subsubsection{Initial data}

To classify the initial data of a bubble in terms of unique parameters is not trivial. Only recently, in Lauterborn et al. (2018) the notions of the energy deposit bubble/ energy deposit case (EDC) (see Fig. 3.1b) and the dimensionless distance $D^{*}$ to the flat, solid boundary were first defined and standardized. Till then, most authors defined their own dimensionless numbers and definitions to categorize their findings for bubble dynamics. The finding that led to the introduction of the EDC is that the bubble dynamics differ to a significant extent depending on whether the bubble expansion phase is included in the simulation or the simulation is started right away from the bubble at maximum expansion (the latter being the so called Rayleigh case (Lauterborn et al. 2018) (see Fig. 3.1a)). Energy deposit literally means that a bubble with a certain energy due to compression is put into the liquid bulk. This resembles a laser generated bubble few nanoseconds after generation, when the plasma of the laser-breakdown has vanished. Throughout this thesis, only energy deposit bubbles are studied.

a)

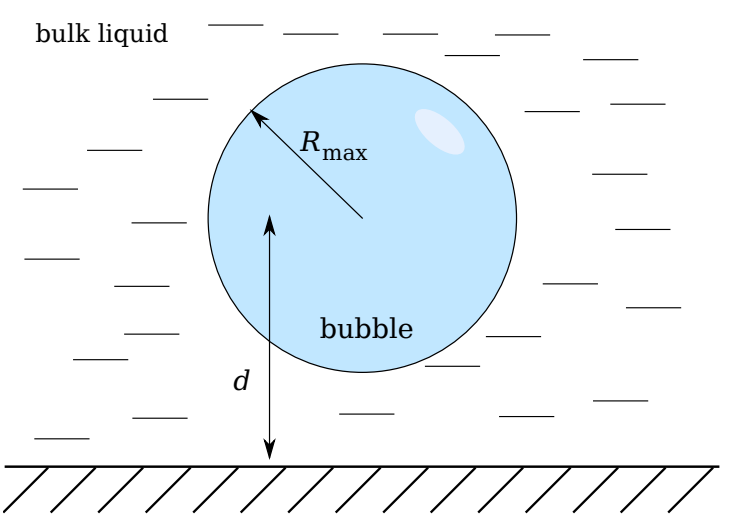

b)

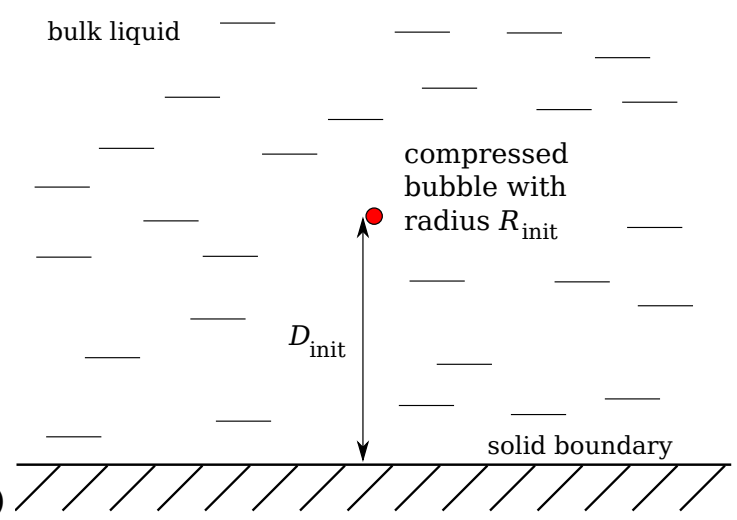

Fig. 3.1: Correction of initial data for a bubble close to a solid boundary (Lauterborn et al. 2018). The bubble starting from maximum radius (a) yields non-physical dynamics (Rayleigh case). For correct modeling the Energy Deposit Case (EDC) (b) is adopted, where the compressed bubble first expands and deforms. 
The normalized distance of the bubble to the flat, solid boundary is an important quantity to classify bubble dynamics phenomena. Historically, it was defined as

the distance of the bubble centre to the boundary divided by the maximum radius

(see e.g. Lauterborn and Kurz (2010), Eq. 34). Rarely, however, it was stated when to take the distance, even though the bubble travels during its lifetime towards the solid boundary. This is because most evaluations employed the Rayleigh case, where the distance is simply the distance at $t=0$, being then the same as the distance at maximum expansion. For small distances, however, firstly the bubble expands aspherically, secondly it travels towards the solid boundary and thirdly the maximum bubble radius differs compared to the one in the unbounded liquid, thus making the historical definition obsolete for small values. The unique definition defined in Lauterborn et al. (2018) is

$$
D^{*} \equiv D_{\text {init }} / R_{\text {max,unbound }}
$$

with $D_{\text {init }}$ denoting the distance at bubble generation, which would be the plasma center in the experiment, and $R_{\text {max,unbound }}$ denoting the maximum radius the bubble would attain in an unbounded liquid. This definition is unique but implies difficulties for a pure experimental study, since the very same bubble cannot be put the into an unbounded liquid in reality. For this thesis this is of no issue because every experiment is followed by a numerical simulation.

So far the theory behind $D^{*}$ is clarified. In this thesis, however, a new difficulty is found, when it comes to numerical precision. When the simulation is set up, a tiny gas bubble has to be resolved spatially by the mesh. The more cells are used to resolve the bubble, the higher the total amount of the cells in the mesh, the higher the computation time. The less cells are used, the more castellated the initial bubble shape looks and therefore the volume differs from the expected one. At that stage the value of the gas pressure $p_{g}$ is in the range of Megapascal, hence small changes in the bubble volume result into a noticeable change in the bubble energy, hence in the maximum radius, hence into $D^{*}$. The question arises, what initial data defines the course of the bubble, meaning the volume over time, usually expressed in terms of the volume equivalent radius $R_{\text {equiv. }}$ over time. Three approaches are used in this thesis, given in Tab. 3.1. 


\begin{tabular}{|c|c|c|c|}
\hline $\begin{array}{l}\text { input pa- } \\
\text { rameters }\end{array}$ & \multicolumn{3}{|c|}{$\begin{array}{l}D^{*} ; R_{\text {init }} ; R_{n 1} ; R_{n 2}<R_{n 1} ; \text { time interval where } R_{n 1} \longmapsto R_{n 2} ; R_{\text {max,unbound }} \text { known } \\
\text { from simulation in unbounded liquid with same parameters; } D_{\text {init }} \text { is calculated from } \\
D^{*} \text { and } R_{\max , \text { unbound. }}\end{array}$} \\
\hline & algorithm 0 & "algorithm 1 & algorithm 2 \\
\hline step 1 & \multicolumn{3}{|c|}{$p_{g}=p_{n}\left(\frac{R_{\text {init }}^{3}-\beta R_{n 1}^{3}}{R_{\text {init }}^{3}-\beta R_{n 1}^{3}}\right)^{\gamma}$} \\
\hline step 2 & \multicolumn{3}{|c|}{ setup mesh } \\
\hline step 3 & $\begin{array}{l}\text { make gas phase where } \\
\text { mesh cell centers are } \\
\text { in theoretical sphere } \\
\text { with radius } R_{\text {init }}\end{array}$ & $\begin{array}{l}\text { make gas phase where mesh cell } \\
\text { centers are in cylinder that aligns } \\
\text { with cell edges and has volume } \\
\text { similar to theoretical sphere with } \\
\text { radius } R_{\text {init }}\end{array}$ & $\begin{array}{l}\text { make gas phase where } \\
\text { mesh cell centers are in } \\
\text { theoretical sphere with } \\
\text { radius } R_{\text {init }}\end{array}$ \\
\hline step 4 & - & $\begin{array}{l}\text { calculate cylinder volume } V_{c} \text { and } \\
\text { from there the new } R_{\text {init }}\end{array}$ & - \\
\hline step 5 & - & $\begin{array}{l}\text { re-calculate } p_{g} \text { with Eq. from step } \\
1 \text { with new } R_{\text {init }}\end{array}$ & - \\
\hline step 6 & - & $\begin{array}{l}\text { get true volume } V_{t} \text { of discretized } \\
\text { gas phase from mesh and from that } \\
\text { the true } R_{\text {init }}\end{array}$ & $\begin{array}{l}\text { get true volume } V_{t} \text { of } \\
\text { discretized gas phase } \\
\text { from mesh and from that } \\
\text { the true } R_{\text {init }}\end{array}$ \\
\hline step 7 & - & $\begin{array}{l}\text { re-calculate } p_{g} \text { with Eq. from step } \\
1 \text { with new } R_{\text {init }}\end{array}$ & $\begin{array}{l}\text { re-calculate } p_{g} \text { with } \\
\text { Eq. } 2.22 \text { with new } R_{\text {init }}\end{array}$ \\
\hline step 8 & - & - & $\begin{array}{l}\text { adapt } R_{n 1} \text { such that } \\
E_{\text {tot,new }}=E_{\text {tot,step1 }} \text { with } \\
\text { an iterative Newton } \\
\text { stepping }\end{array}$ \\
\hline step 9 & \multicolumn{3}{|c|}{ apply $p_{g}$ to gas phase } \\
\hline $\begin{array}{l}\text { during } \\
\text { simula- } \\
\text { tion }\end{array}$ & \multicolumn{3}{|c|}{$\begin{array}{l}\text { during specified time interval linearly reduce } R_{n 1} \text { to } R_{n 2} \text { via global } \\
\text { mass correction to ensure strong collapse }\end{array}$} \\
\hline
\end{tabular}

Table 3.1: Specification of the three approaches used in this thesis intended to ensure that the same input parameters yield the same bubble dynamics roughly independent of mesh resolution. 


\subsubsection{Meshes}

Simulating a cavitation bubble is very demanding for the mesh used. As partly stated in Koch et al. (2016) already, the mesh has to satisfy the following criteria:

Criteria 3.1. Demands for a mesh

1. resolve the initial bubble $\left(R_{0} \approx 20 \mu \mathrm{m}\right)$

2. resolve the dynamics in the bubble region $(R \in[0 \mathrm{~mm}, 1 \mathrm{~mm}])$

3. keep the outer boundary approximately $100 R_{\max }$ away from the bubble for unbounded and semi-unbounded simulations because of the far reaching influence of the pressure profile generated by the bubble

4. resolve the shockwave in the bubble region but dissipate it further outwards, so that least possible reflections occur

5. areas of different resolution seem to need a smooth transition (this is a qualitative conclusion from experience and still needs to be quantified - it limits the application of e.g. dynamic/ static mesh refinement)

6. the cells need to be aligned as good as possible with the bubble interface, especially at maximum bubble volume, when surface tension is set non-zero in order to avoid amplifcation of parasitic currents (Harvie et al. 2006).

7. mesh quality criteria have to be fulfilled as good as possible, meaning that

- the edge aspect ratio of each of the cells needs to be close to 1 ,

- the connecting line between the centroids of neighbouring cells needs to pass through a point on the face the cells share that is close to the face center, i.e. the cells must not be skew

Point 3 and 4 are quantified in Sec. B.1.1 concerning the convergence of the solution.

In the following sections the meshes used in this thesis are presented and stated how the above criteria are incorporated.

\subsection{2.a Axial symmetry, polar cell orientation, unbounded liquid and bubble close to a solid boundary}

A similar type of mesh has been introduced already in Koch et al. (2016). The parameters and details since then have constantly been adapted with further experience. The idea is to align the cells as good as possible to the bubble interface (Crit. 3.1, point 6), i.e. a polar orientation with apex at the initial bubble center, while at the same time dissipate the shockwave in the outer regions and avoid a high total cell amount for the whole mesh. A sketch of the mesh is given in Fig. 3.2. The mesh center core region $(\mathcal{C}=\{x \in[0, \mathrm{Xi}], y \in[-\mathrm{Xi}, \mathrm{Xi}]\})$ consists of a rectangular block with cells in Cartesian orientation and homogeneous minimum cell size. This cell size is also the criterion the convergence of the solution is tested on in the Sec. B.1.1 and Sec. B.1.3. The follow-up region $\left(\mathcal{X}_{i i}=\{r<\mathrm{Xii}\} \backslash \mathcal{C}\right)$ serves as the transition region from Cartesian to polar cell orientation. Usual values are $1.2 \cdot \sqrt{2} \mathrm{Xi} \leq \mathrm{Xii} \leq 2 \mathrm{Xi}$. The next region 
$\left(\mathcal{X}=\{r<\mathrm{X}\} \backslash\left(\mathcal{C} \cup \mathcal{X}_{i i}\right)\right)$ consists of cells with edge aspect ratio almost equal to 1 . Because of the spreading in $\varphi$-direction, the cell closest to $\mathrm{X}$ must have an edge length $\delta x_{\mathrm{X}}$ that is by a factor of $g_{f 1}=\mathrm{X} / \mathrm{Xii}$ larger than the edge length $\delta x_{\mathrm{Xii}}$ of the cell closest to Xii (Koch 2014). $g_{f 1}$ is called grading. In the last region $\left(\mathcal{X}_{F}=\{\mathrm{X} \leq r \leq \mathrm{XF}\}\right)$ the grading is exaggerated by a user defined grading factor $g_{f}$, usually chosen to be 5.2. Standard parameters for this mesh would be:

$$
\mathrm{Xi}=50 \mu \mathrm{m}, \mathrm{Xii}=2 \cdot \mathrm{Xi}, \mathrm{X}=1.05 R_{\max }, \mathrm{XF}=100 R_{\max }, g_{f}=5.2
$$
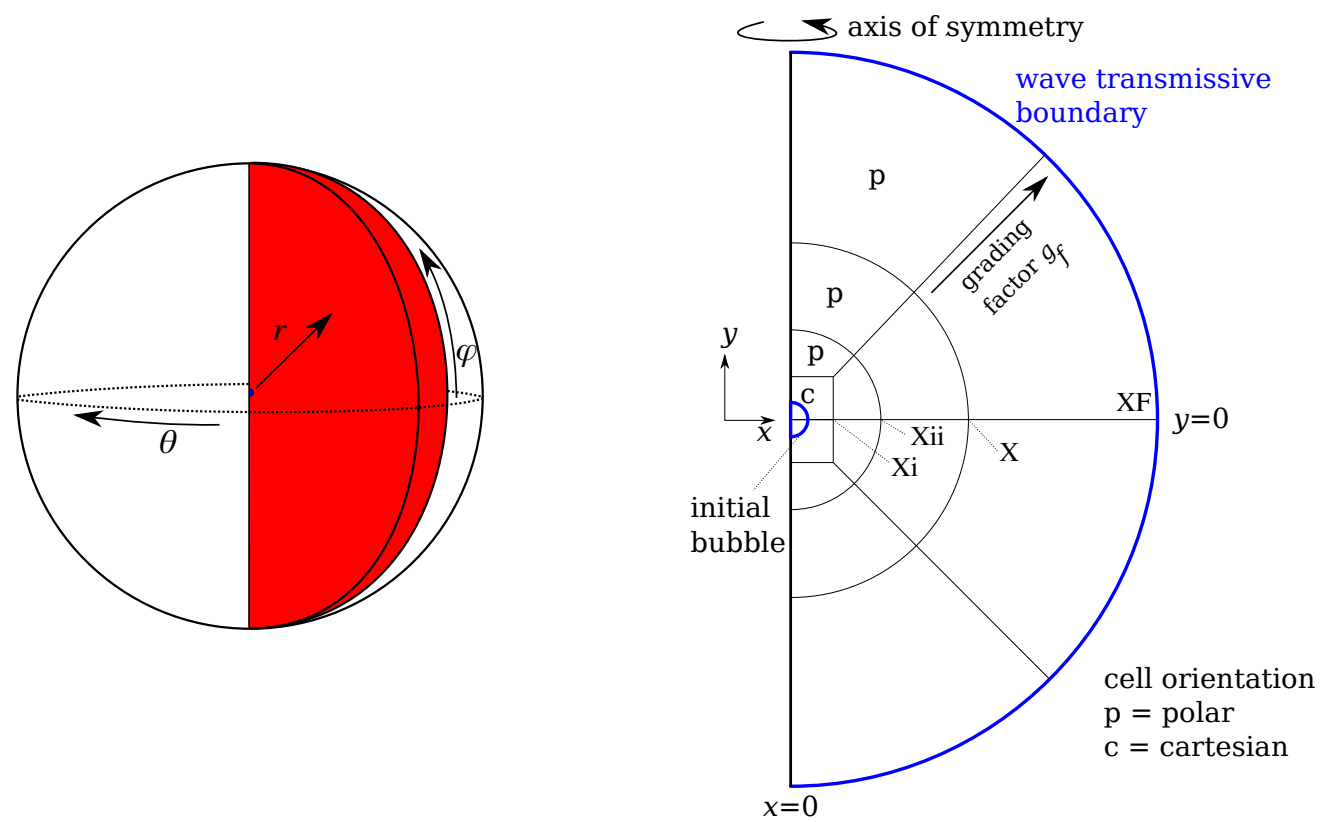

Fig. 3.2: Sketch of the mesh with axial symmetry and polar cell orientation. Not true to scale.

For a bubble close to a solid boundary this mesh is also used. The lower part below the bubble can be cut away at the preferred distance by applying the snappyHexMesh-tool to the mesh, defining the cut-away part by a box covering it (sketch see Fig. 3.3). snappyHexMesh is a parameter-rich tool. The configuration file that does the rather simple task of cutting the lower part of the mesh is provided in the Repos. B.3, too.

apply snappyHexMesh
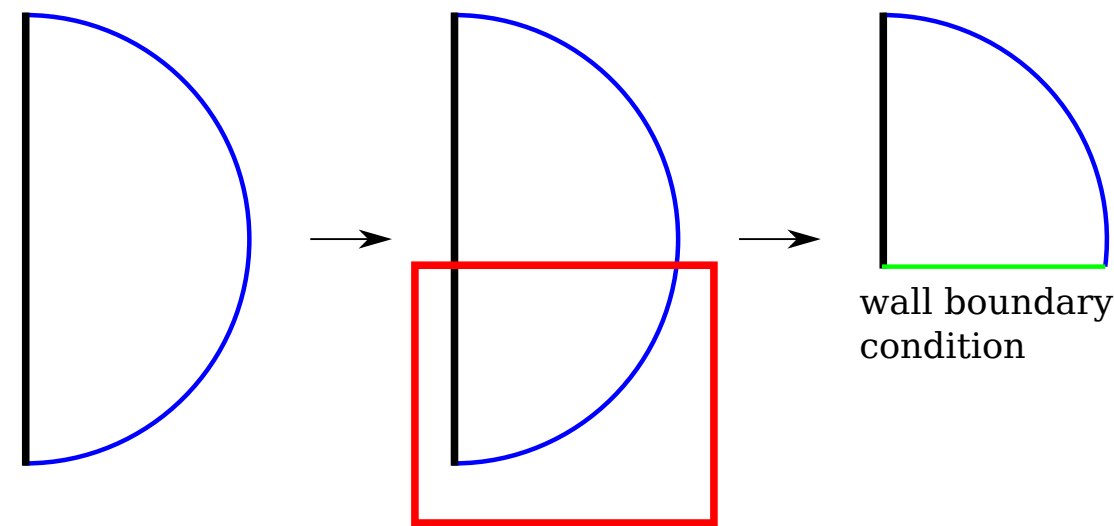

Fig. 3.3: Cutting the mesh for unbounded liquid to a mesh with a solid boundary. 


\subsection{2.b Spherical symmetry}

In order to be able to calculate an unbounded bubble with the least computational effort, a mesh with spherical symmetry was created (sketch in Fig. 3.4). The amount of cells in both $z$ and $y$-direction is only 1 and the top, bottom, front and back boundaries are given the wedge boundary condition. Only the outermost side at $x=\mathrm{XF}$ in version $\mathrm{A}$ ) and $x=\mathrm{XFFF}$ in version B) is given the wave transmissive boundary condition. Mesh version B) includes a region $\left(\mathcal{X}_{F F F}=\{\mathrm{XFF} \leq x \leq \mathrm{XFFF}\}\right)$ for stronger dissipation of the shockwave emitted by the bubble. $\mathrm{X}$ in version $\mathrm{A}$ ) and $\mathrm{XF}$ in version $\mathrm{B}$ ) are chosen such that their values are bigger than $R_{\max }$. The same parameters for the Mesh 3.1.2.a can be used for this mesh version A), too.

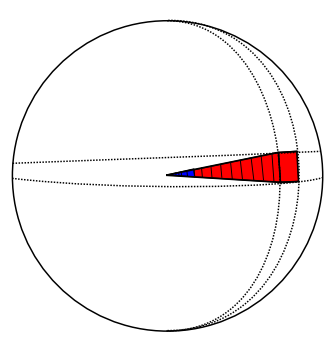

A)

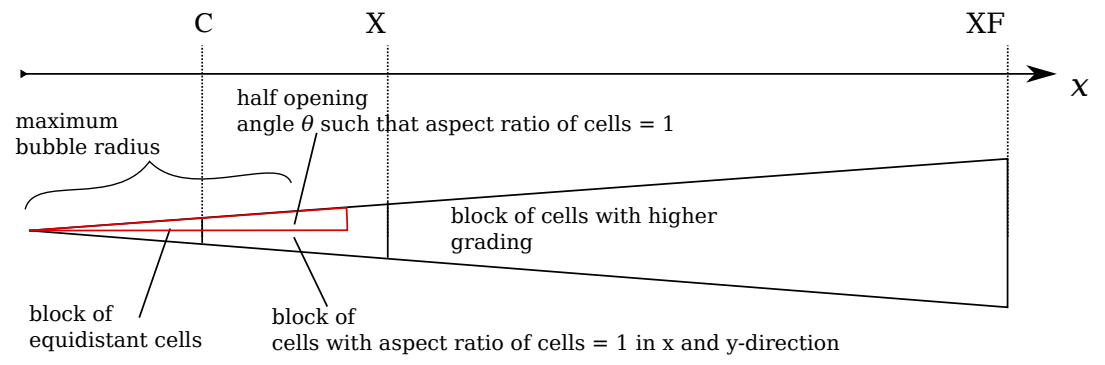

B)

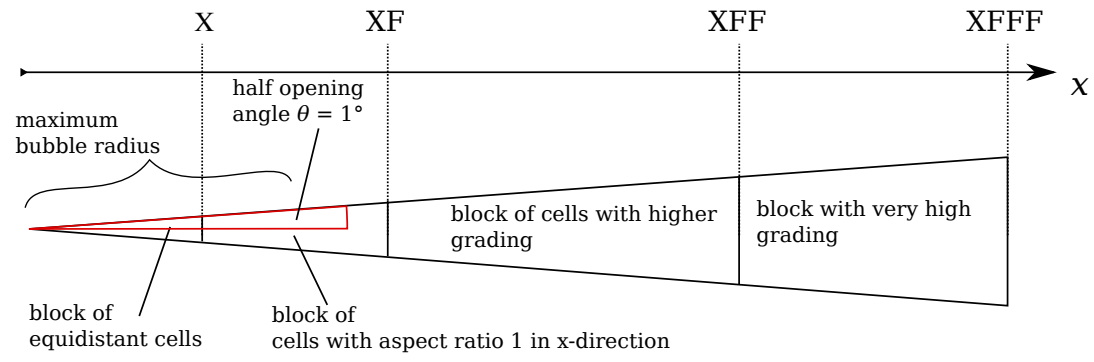

Fig. 3.4: Sketch of the mesh with spherical symmetry in two different versions A) and B). Not true to scale.

\subsection{2.c Axial symmetry, Cartesian cell orientation, concentric refinements towards bubble region}

Recently, static mesh refinement was rediscovered as a meshing means in the working group. Static refinement is the process of doubling the spatial resolution in defined areas/volumes of the already set-up mesh, before starting the simulation. These refinements can be cascaded.

Static refinement was discarded years ago because pressure wave reflections at the resolution jump were observed. Since then a lot of work and experience on and with the solver and solution control parameters, as well as software updates have passed. Nowadays, static refinement doesn't seem to show pressure wave reflections or interface deviations as clearly as back then anymore. Whether these issues are fully or partly gone is still to be extensively tested. If it turns out to be as useful as the polar mesh approaches, thereby making Crit. 3.1 point 5 unnecessary, it would be very helpful, because setting up a mesh with static refinement needs a lot less effort than other types of meshes. A sketch is given in Fig. 3.5. One more advantage is that all cells are aligned in a Cartesian way and have an edge to edge aspect ratio of close to 1, which is not the case in most other meshes (Crit. 3.1 point 7). The aspect ratio is an important measure for 
error estimation. One disadvantage is that spurious/ parasitic currents (small numerically induced vortices (Harvie et al. 2006)) at the bubble interface may occur during maximum bubble expansion where the interface velocity is temporarily low, because the interface is less aligned with the mesh than it would be in a polar mesh (Crit. 3.1 point 6 ).
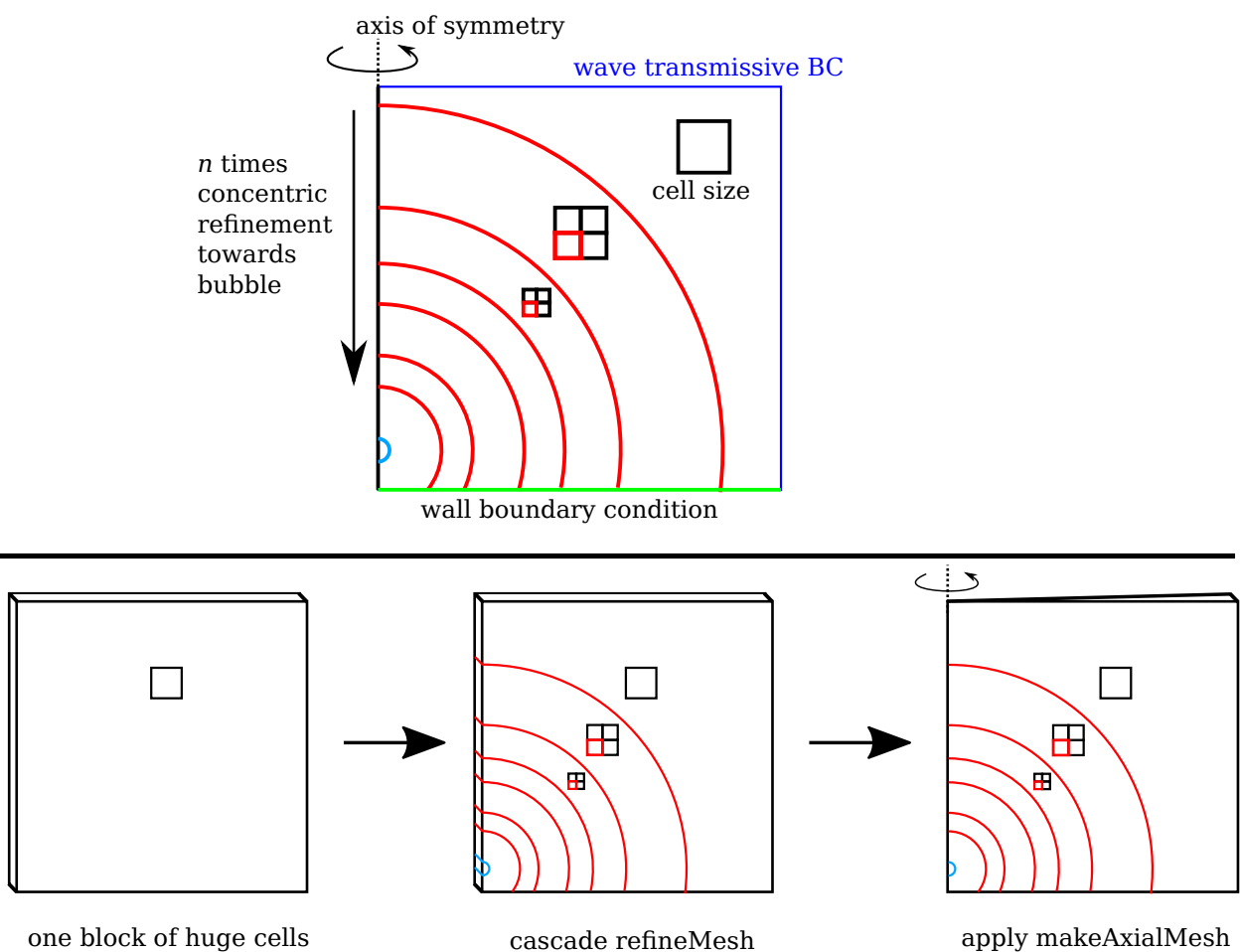

cascade refineMesh

apply makeAxialMesh

Fig. 3.5: Sketch for the setup procedure of the axisymmetric mesh with concentric cell refinement areas. Not true to scale.

Two ways of defining the refine $n$ radii, $r_{\text {refine }, j}$, have been employed. The first one, following the ambition to automatize the meshing process for any value of $n$, a formula has been created with the minimum and maximum refine radius, $r_{\text {refine,min }}$ and $r_{\text {refine,max }}$, as input parameters:

$$
r_{\text {refine }, j}=\left(r_{\text {refine,max }}-r_{\text {refine,min }}\right) \cdot\left(\frac{(n+1)-j}{n}\right)^{2}+r_{\text {refine,min }}, \quad j \in[1,2, \ldots, n]
$$

The second one fixes $n=10$ and scales the $r_{\text {refine, } j}$ with $R_{\max }$ :

$$
\begin{aligned}
& r_{\text {refine }, j}=a_{j} R_{\max }, j \in[1,2, \ldots, 10] \\
& a_{1}=55, a_{2}=15, a_{3}=9, a_{4}=6, a_{5}=3, \\
& a_{6}=2, a_{7}=1, a_{8}=0.8, a_{9}=0.5, a_{10}=0.2
\end{aligned}
$$

\subsection{2.d Full 3D bubble close to the solid boundary}

The full 3D mesh was created for a few trials in 3D. Since the energy deposit bubble needs to be calculated including the expansion phase, calculation in 3D takes massive computation resources. Aligning the cells to the bubble interface is a lot more complex in 3D than in axial symmetry. The sketch is given in Fig. 3.6. 


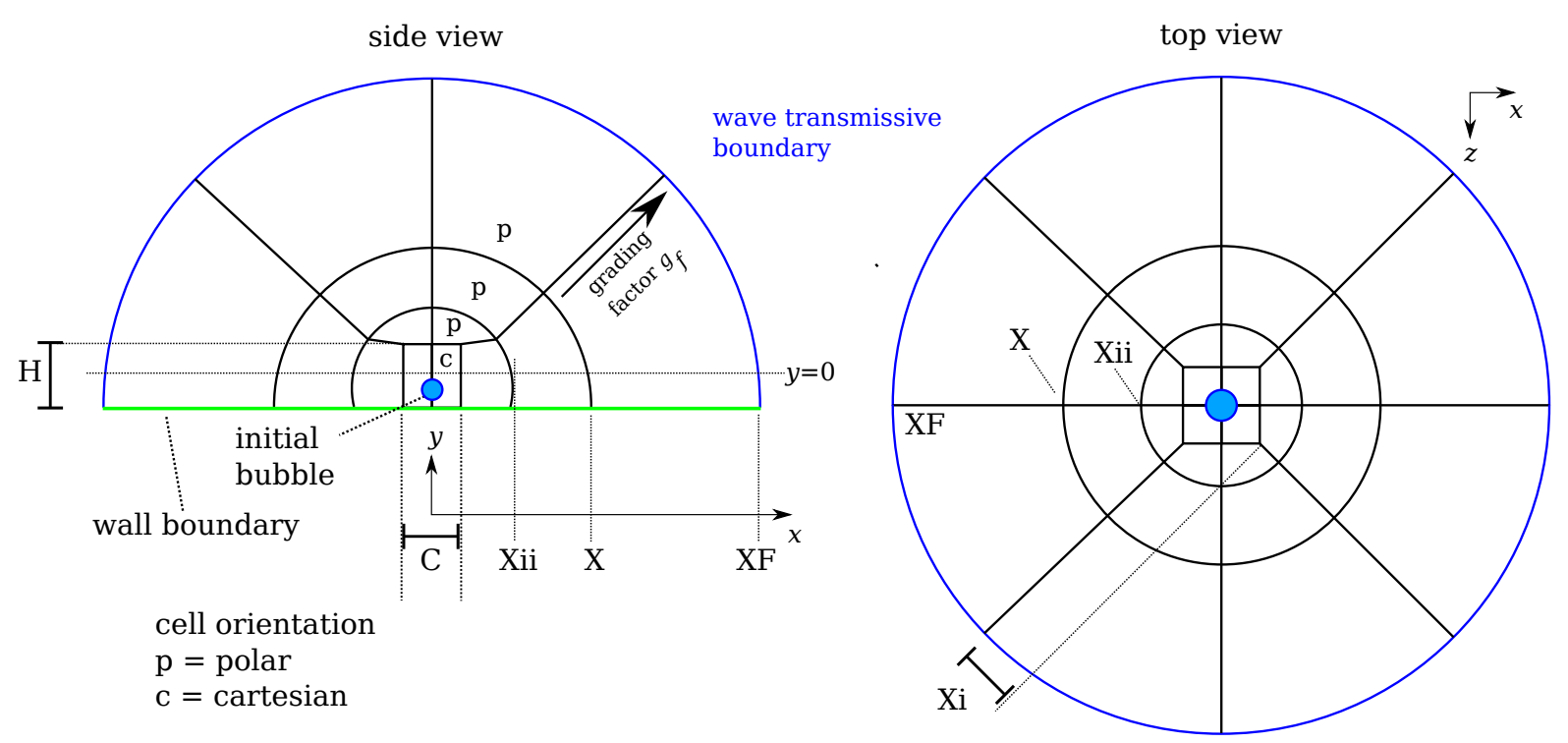

Fig. 3.6: Sketch for the mesh for the calculation in full 3D. Not true to scale.

In contrast to the axisymmetric polar mesh, the Cartesian core is rectangular $(\mathrm{H}>\mathrm{C})$ rather than cubic, in order to increase the resolution at $y \approx 150 \mu \mathrm{m}$, where the fast jet is generated for $D^{*} \leq 0.2$.

Standard parameters are:

$$
\mathrm{C}=40 \mu \mathrm{m}, \mathrm{H}=180 \mu \mathrm{m}, \mathrm{Xii}=1.1 d_{H}, \mathrm{X}=1.2 R_{\max }, \mathrm{XF}=80 R_{\max }, g_{f}=1
$$

with $d_{H}=\sqrt{\mathrm{Xi}^{2}+\mathrm{H}^{2}}$ the diagonal from bottom center to top corner of the Cartesian core. Strictly speaking, $\mathrm{XF}=80 R_{\max }$ is too less comparing Crit. 3.1 point 3 , but the fact that the angular resolution in polar and azimuthal direction is maintained throughout the mesh, led to choosing a lesser value for saving cells. A cell size of $\delta x_{\min }=1.8 \mu \mathrm{m}$ in the Cartesian core leads to a total amount of cells with these parameters of 6,407,940. $g_{f}$ could be increased still, but with an azimuthal resolution of

$$
\operatorname{res}_{\mathrm{azi}}=\frac{2 \mathrm{C}}{\delta x_{\min }} \operatorname{per} 180^{\circ}
$$

the cell number in radial direction in the interval $\mathrm{X} \leq r \leq \mathrm{XF}$ becomes quite low, producing excessive cell edge ratios when over-driving $g_{f}$.

\subsection{2.e Quarter cake - 3D with cyclic boundaries}

In order to calculate quasi 3D but save time, a quarter 3D mesh was created using cyclic boundaries. With this mesh it was possible to approach a quarter-3D convergence study. A sketch is given in Fig. 3.7. 

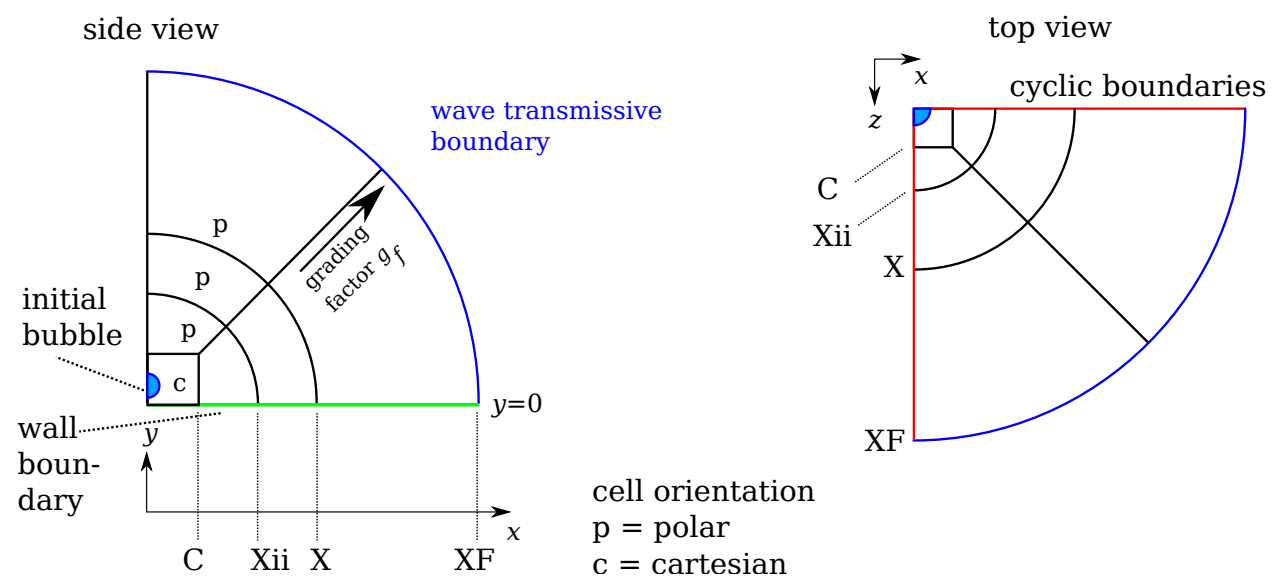

Fig. 3.7: Sketch for the quarter 3D calculation with cyclic boundaries. Not true to scale. Note the distances labeling is different to the other meshes in the inner part.

The standard parameters employed for this mesh were:

$$
\mathrm{C}=80 \mu \mathrm{m}, \mathrm{Xii}=1.6 \sqrt{3} \mathrm{C}, \mathrm{X}=1.2 R_{\max }, \mathrm{XF}=80 R_{\max }, g_{f}=5.25
$$

The reason for the outer boundary distance of $80 R_{\max }$ again violating Crit. 3.1 point 3 is that the value was taken over by the studies in full 3D. However, here the azimuthal resolution (Eq. 3.4) is doubled compared to the full 3D simulation because of $\mathrm{C}_{q c}=2 \mathrm{C}_{3 D}$, hence the amount of cells in radial direction also increases. That is why $g_{f}$ could be increased again.

\subsubsection{Time stepping}

The time stepping is explained in Sec. 2.4.1. The standard values for the calculations are given there, too.

\subsubsection{Grid convergence study and best practice meshing}

After having described the numerical method, the initial data and the meshes, the next logical step is to make sure that the solution converges. Numerous investigations with varying solution control parameters have been performed on the single bubble in unbounded liquid in both spherical and axial symmetry. However, in order to keep the story-line of the thesis, these have been put into the appendix Sec. B.1. Here, only the results are given, which lead to solution convergence of a bubble in unbounded liquid with initial data:

$$
R_{\text {init }}=20 \mu \mathrm{m}, R_{n, 1}=184.1 \mu \mathrm{m}, R_{n, 2}=64 \mu \mathrm{m}, R_{n} \text { reduction in interval }[60 \mu \mathrm{s}, 75 \mu \mathrm{s}]
$$

The time from bubble generation $(t=0)$ and the first minimum bubble volume $\left(t\left(V_{\min }\right)\right)$ is taken as the criterion for convergence. In unbounded liquid and for laser generated bubbles the duration for expansion and collapse is the same, thus the convergence criterion is called $2 T_{c}$. This quantity behaved in an unexpected way with resolution refinement in the first place. Numerous solution control parameters have been studied until finally the solution converged. The following criteria for solution convergence could be deduced from the studies, in addition to the Crit. 3.1: 
Criteria 3.2. best practice guidelines for solution convergence

1. Adapt the initial data $\left(p_{0}, R_{n}\right)$ by the bubble volume $\left(V_{0}\right)$ resulting from the initial discretization such that either i) the initial potential Energy of the theoretical values is maintained, or ii) the adiabatic relation $p_{0} R_{\text {init }}^{\gamma}=p_{n} R_{n}^{\gamma}$ is fulfilled (see algorithms in Tab. 3.1)

2. Smearing out the interface over 3 cells in the initial data already doesn't seem to have a positive effect for low resolutions, therefore keep the interface sharp

3. The resolution is highest in the bubble region and is coarsened succesively towards a distance to the bubble of $\geq 100 R_{\max }$

4. Consider the traveling time of the shockwave from bubble generation from the bubble to the outer boundary and back. Setting the outer boundary too close, the broadened wave reflection will hit the bubble maybe during its maximum expansion phase and thus influencing the bubble. The influence then becomes stronger with better resolution because the shockwave is less damped. For a bubble of $R_{\max } \approx 500 \mu \mathrm{m}$ it is advisable to set the outer boundary $\geq 100 R_{\max }$ apart from the bubble. In the study cases for the convergence it has been found out that setting it only $80 R_{\max }$ apart from the bubble leads to non-convergence of the solution. In Koch et al. (2016) it was shown that for the sake of resolving the static pressure profile, a distance $\geq 100 R_{\text {max }}$ irrespective of the value of $R_{\max }$ is necessary, too, in order to simulate an unbounded bubble.

The two reference solutions are summarized in Fig. 3.8.

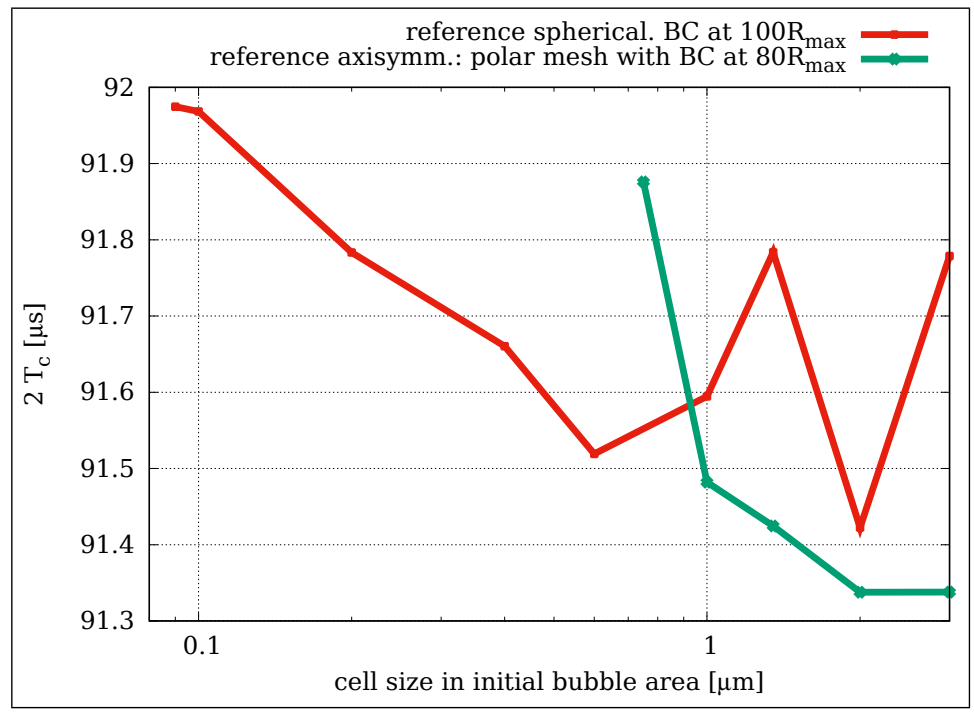

Fig. 3.8: Time from bubble generation till first minimum volume for different resolutions for the two reference solutions for the unbounded bubble. Axisymmetric calculation (green) of Fig. B.1 and converged spherical calculation (red) of Fig. B.3

\subsubsection{Correlate $R_{n}$ and $R_{\max , \text { unbound }}$}

The bubble in unbounded liquid with initial data

$$
R_{\text {init }}=20 \mu \mathrm{m}, R_{n, 1}=\text { various, } R_{n, 2}=64 \mu \mathrm{m}, R_{n} \text { reduction in interval }[60 \mu \mathrm{s}, 75 \mu \mathrm{s}] \text {, }
$$


is investigated in spherical symmetry with the Mesh 3.1.2.b (version A) with the properties

$$
\delta x_{\min }=1 \mu \mathrm{m}, \mathrm{C}=80 \mu \mathrm{m}, \mathrm{X}=1.2 \cdot 500 \mu \mathrm{m}, \mathrm{XF}=100 \cdot 500 \mu \mathrm{m}, g_{f}=5.25 .
$$

With this configuration the direct link between $R_{n, 1}$ and $R_{\text {max,unbound }}$ can be calculated (Fig. 3.9).

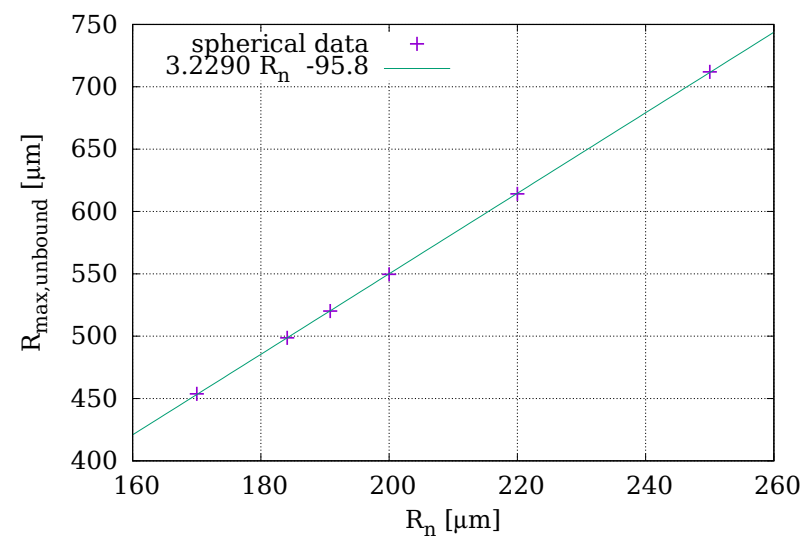

Fig. 3.9: Correlation between $R_{n, 1}$ and $R_{\text {max,unbound }}$.

The fitted relation reads:

$$
R_{\text {max,unbound }}[\mu \mathrm{m}]=3.2290 R_{n, 1}[\mu \mathrm{m}]-95.8
$$

\subsection{Overlay method: ray-tracing to bring numerics and experiments to an overlay}

(This section is similar to parts in Koch et al. (2020). An updated, more detailed and more quantitative investigation is found there.)

Designing advanced experiments needs substantial planning and also knowledge of what results to expect. Testing different configurations can be costly and time consuming, and methods to help in this respect may be welcome. When simulations of two-phase flows and experiments with imaging cameras are involved, a special blend of the visualization of numerical results and the images from the experiment may substantially improve the outcome by taking into account the omnipresent refraction at phase boundaries. Vice versa, the experimental observations could be optimized by analyzing optical ray paths in the arrangement and by later inserting numerical simulations into the ray-tracing engine used. Then the experimental photograph can directly be compared with the simulated image. The abstract concept will become more clear by an example: a bubble in water.

The idea is to generate an image from the results of a (two-phase) computational fluid dynamics simulation that looks most alike the one obtained from the experiment in order to bring both to an overlay. Assuming that the geometry and dimensions of the experiment including the illumination devices are known, the procedure for the numerical side would then be the following:

Firstly, the closed interface iso-plane of the two fluid phases from the numerical CFD simulation has to be extracted to a standard 3D format. For example, most programs are suited with an import/export function to the st 1 format. Secondly, the st 1 file obtained can be imported into a program with a realistic, light-ray-tracing engine. A variety of specialized raytracing programs might exist. Here, the free, open-source project blender is taken because 
it incorporates a very realistic and physical lighting engine, standard st 1 import compatibility, a python-language application programming interface (API) and a large variety of 3D editing tools. The latter become important when it comes to modelling the experimental setup, and the API is handy for batch operations on many simulation time steps and parameter scans. Note that the aim is to produce a realistic image from the CFD simulations rather than perform scientific analyses of optics. Thus interference can be neglected, but the intensity and diffusivity of the refraction and reflection at objects matter. The following steps were performed with blender in order to achieve a realistic image:

The cycles render engine is used (Blender Foundation and Community v. 2.82). This engine emits the light rays from the camera into the 3D scene and distinguishes between so called camera rays, reflected rays, transmission rays and shadow rays. After importing the st 1 geometry object, its surface is both smoothed and reduced in complexity by applying the limited dissolve algorithm. This algorithm accounts for reducing the amount of faces while keeping the same shape. This st 1 geometry is then given a material with an index of refraction (IOR). The so called GlassBSDF material with an index of refraction of 0.75 suits best for an air bubble in water. Depending on the direction of the face normal of the edited st 1 geometry object, the IOR ratio either has to be set to 1.333 or $1 / 1.333=0.75$.

The optically relevant geometries of the experiment then are created around the bubble geometry along with their optical properties of, e.g., glossiness, light transmission or light emission. Simple diffusive, glossy, glass-like or emissive materials do the work in most cases to mimic optically relevant lab equipment. The water of the cuvette is mimicked by a block given the GlassBSDF material with an index of refraction of 1.333. This block can be seen in 3.11 and Figs. 3.16b,c. The solid boundary made of glass, where the bubble collapses to in the experiment, is modelled by a simple block object of the GlassBSDF material with an index of refraction with respect to water of $1.333 / 1.45=0.92$. The flash tube geometries can be designed adequately and a simple emissive material can be attached to them. The ray-tracing camera can be set up essentially with the same properties as in the experiment concerning focal length, sensor size and pixel resolution.

\subsubsection{Validation of the ray-tracing engine}

(This section is similar to parts in Koch et al. (2020). An updated, more detailed and more quantitative investigation is found there.)

Experimental setup: Static bubble - In order to validate the blender ray-tracing engine, an experiment was chosen, where a bubble of less than a millimeter in diameter rests fixed on a microliter syringe needle (Fig. 3.10). This static bubble then has a very low contact area to the needle and is therefore almost perfectly spherical in shape. This scene can be mimicked in the blender software as shown in Fig. 3.10, right. 


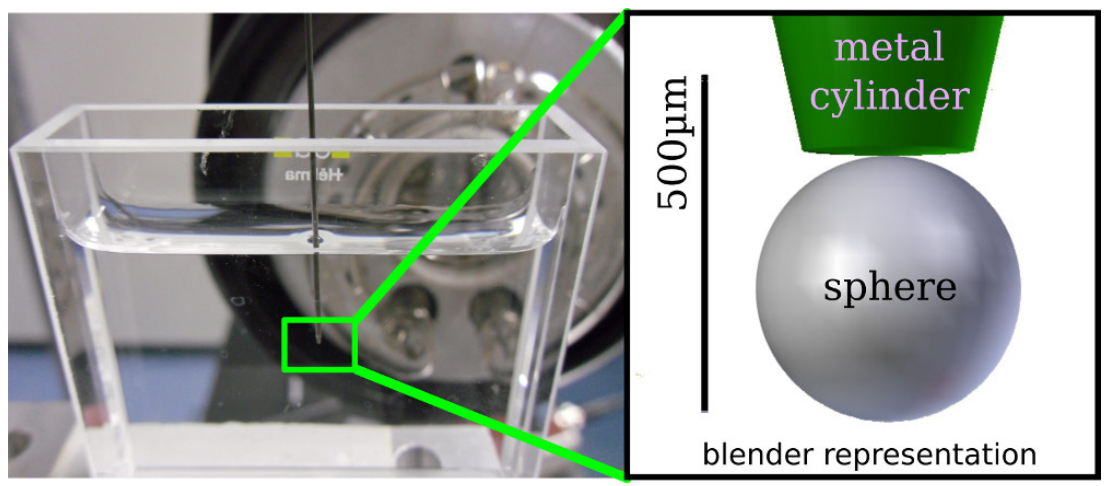

Fig. 3.10: Comparison of the experimental arrangement (photograph), left, and corresponding numerical configuration for blender, right, of a static, spherical bubble to validate the blender ray-tracing engine.

The complete experimental setup consists of essentially six elements, as shown in Fig. 3.11: (i) A water filled glass cuvette with inner dimensions $5 \mathrm{~cm} \times 1 \mathrm{~cm} \times 4 \mathrm{~cm}$ in width, depth and height; (ii) a background illumination xenon flash Mettle MT-600DR (see also Sec. C.1) with distance to the bubble of $5.2 \mathrm{~cm}$. (iii) A side illumination xenon photo flash Mecablitz $36 C T 2$ (see also Sec. C.1) with a distance to the bubble $\approx 8.5 \mathrm{~cm}$; (iv) a microliter syringe and needle producing a bubble of about $300 \mu \mathrm{m}$ to $500 \mu \mathrm{m}$ in diameter inside the cuvette; (v) a K2 Infinity microscope objective with a magnification yielding about $2 \mu \mathrm{m} / \mathrm{pixel}$; (vi) and a high speed camera Imacon 468. The items are used in the main experiments, too and are described in more detail in Sec. 3.3.

Results of the comparison - In Fig. 3.12 the comparison of the images of two bubbles from the experiment (left) and the ray-traced sphere in blender (right) is shown. Row a) shows the setup with the Mecablitz in the same position as in Fig. 3.11, while row b) shows the setup with its position rotated clockwise by $45^{\circ}$ in the plane of the setup sketch around the axis of the syringe needle. Clearly seen in in each of the bubbles is the distorted image of the background ring flash tube, as well as the bright line on the outer right rim, caused by total reflection. Due to the asymmetry in the setup this total reflection line is emphasized on the right side of the bubble. Also the influence of the position of the side flash is captured correctly by blender: the rotated position of the flash enhances double refraction. It is seen now as two white lines near the left rim of the bubble. Comment: The picky reader might not agree that the comparison is precise enough. A new, quantitative comparison has been made and published in Koch et al. (2020). 


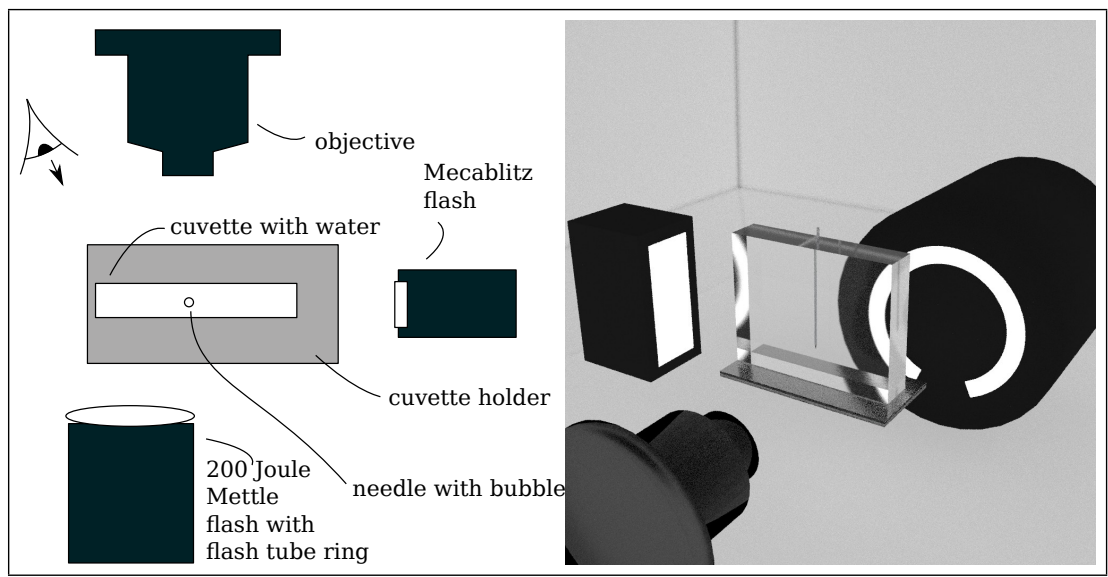

Fig. 3.11: Setup for the static bubble experiment. Sketch of the elements of the experimental arrangement (left) transferred into blender elements (right) from the perspective indicated by the eye in the sketch. Needle and water block are modeled as well in blender.

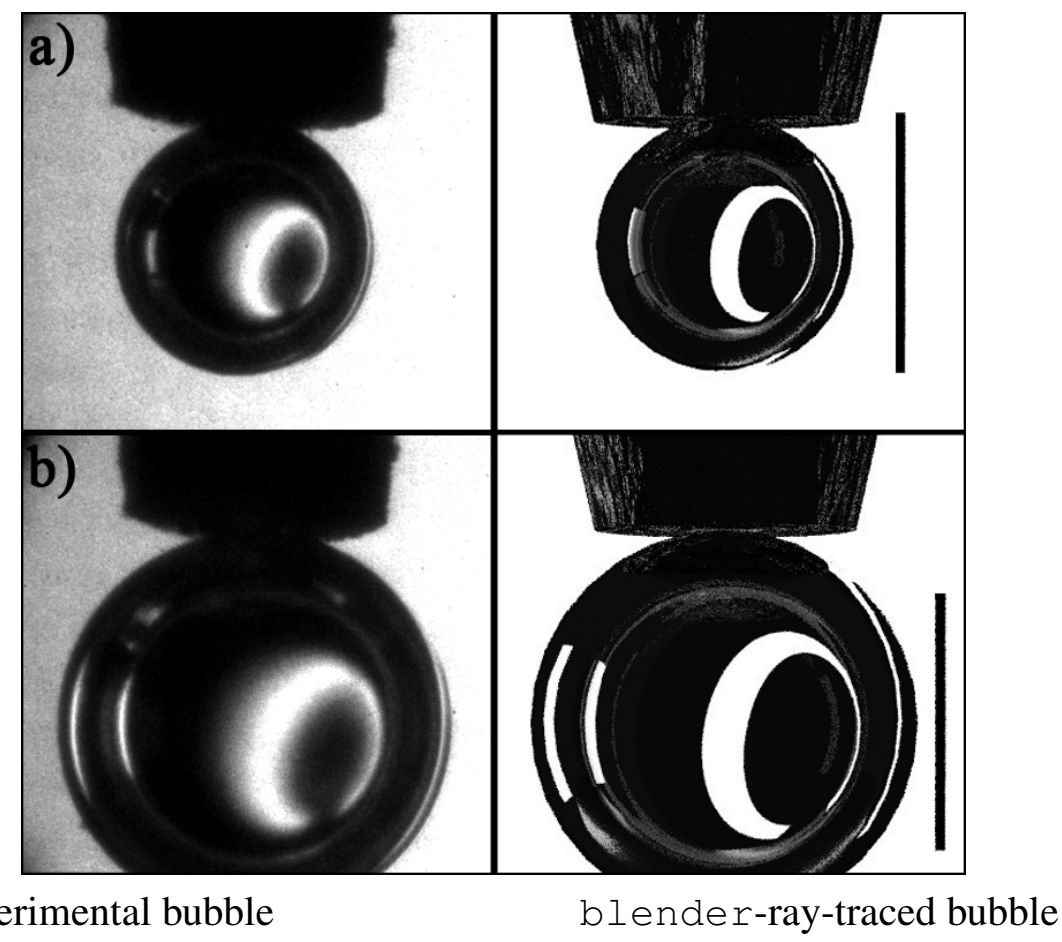

Fig. 3.12: Comparison of the experimentally obtained images (left) of a static, sub-millimeter bubble and their blender-ray-traced representation (right). Top and bottom rows differ by the position of the side flash and bubble size. The bar indicates a length of $250 \mu \mathrm{m}$. 


\subsection{Experiments}

Three different setups were used to gain the experimental results presented in this thesis. From the numerics it is known that the boundary has a strong influence when it's put too close to the bubble. Therefore, in the first place, a setup was created with a very large cuvette $(10 \mathrm{~cm} \times 10 \mathrm{~cm} \times 30 \mathrm{~cm})$. Using this cuvette, results with kiloframes per second time resolution were obtained with the Photron APX-RS high speed camera, showing the full bubble lifetime. Illumination was done by a pulsed, overdriven $100 \mathrm{~W}$ LED. However for higher time resolution it was found that there is no suitable, technical possibility in the world to illuminate the bubble with enough intensity over such a distance. Therefore, the second setup comprised a much smaller cuvette, a Xenon flash and the IMACON 468 camera that takes 8 images at ultra high-speed. This produced valuable results already close to megaframes per second. It was found, however, that the energy jitter of the bubbles was still too high to manage manual triggering to microsecond precision into a certain part of the bubble dynamics. Thus, the third and final setup comprised the second setup including a He-Ne laser beam running through the bubble and hitting a terminal high-speed photodiode. This triggering method was found to date back to Lauterborn and Bolle (1975). With this trigger circuit it was possible to repeatably capture certain dynamics for the so called mushroom bubbles close to a rigid cylinder.

Laser - The single bubble is generated by optical breakdown of a nanosecond laser pulse in water. The laser pulse of wavelength $532 \mathrm{~nm}$ is generated by a Q-switched, frequency doubled Nd:YAG laser (Litron Nano PIV). It is operated with an external triggering circuit at $15 \mathrm{~Hz}$ and a single light pulse out of 20 subsequent pulses is selected by a manual button. The laser pulse duration $t_{p}=10 \mathrm{~ns}$ FWHM was measured with a high speed photodiode (Thorlabs SV2-FC) via manually fitting the following function to the photodiode signal

$$
u(t)=A \exp \left(-\frac{(t-\tau)^{2}}{2\left(\sigma_{F W H M} / \sqrt{8 \ln 2}\right)^{2}}\right)
$$

and taking the full-width-half-maximum as $t_{p}$. The result is shown in Fig. 3.13a. A little variation is found, however that may result from manual fit. Averaging over measurements and automatic fitting was not performed because the intention was only to get an estimate of the pulse duration. In Fig. 3.13b the impulse response of the photodiode is given and manually fit with the same fit function, obtaining a pulse duration of $0.8 \mathrm{~ns}$. The impulse response was measured by exposing the photodiode to a femtosecond laser with $5 \mathrm{MHz}$ repetition rate (courtesy Dr. Ingo Gregor) and the signal was recorded with a 40 Gigasamples per second ( $\equiv 40 \mathrm{~S} / \mathrm{ns}$ ), $2.5 \mathrm{GHz}$ ( $\equiv 0.4 \mathrm{~ns}$ ) bandwidth oscilloscope.

The plasma cross section $A_{\text {plasma }}$ of one of the experiments (see later Fig. 4.17) in the direction of the laser is approximated by taking the half width of the bright plasma as the cross section radius. The cross section then amounts to $A_{\text {plasma }} \approx \pi(12 \mu \mathrm{m})^{2} \approx 450 \mu \mathrm{m}^{2}=4.5 \cdot 10^{-7} \mathrm{~cm}^{2}$. For now it is assumed that the plasma cross section is comparable in all experiments conducted and the laser energy is chosen close to the breakdown threshold, then the laser energy $E_{l}$ leading to breakdown is roughly

$$
E_{l} \approx I_{\text {thr }} \cdot A_{\text {plasma }} \cdot t_{p}=44.5 \mathrm{~mJ} \equiv(22.25 \% \text { of } 200 \mathrm{~mJ}),
$$

the $200 \mathrm{~mJ}$ being the specified total energy of one laser beam pulse. This can be confirmed with the experience that the knob adjusting the laser energy was usually set around $20 \%$ for lowest 
energy producing a bubble.

$44.0 \%, \mathrm{FWHM}=10 \mathrm{~ns}$ $34.3 \%$, FWHM=9.8ns $34.3 \%, \mathrm{FWHM}=10 \mathrm{~ns}$

measurements of the laser pulse with a thorlabs photodiode

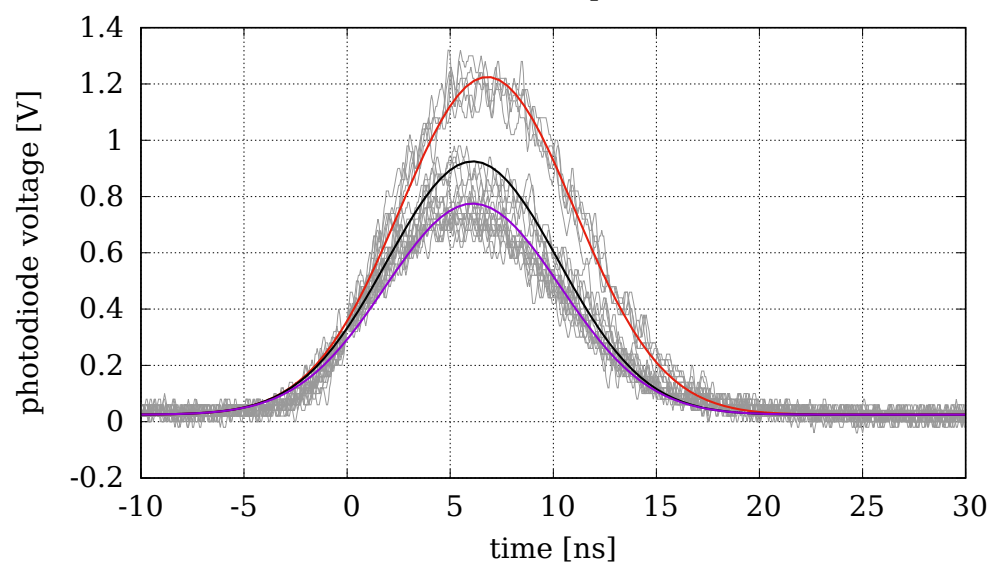

A)

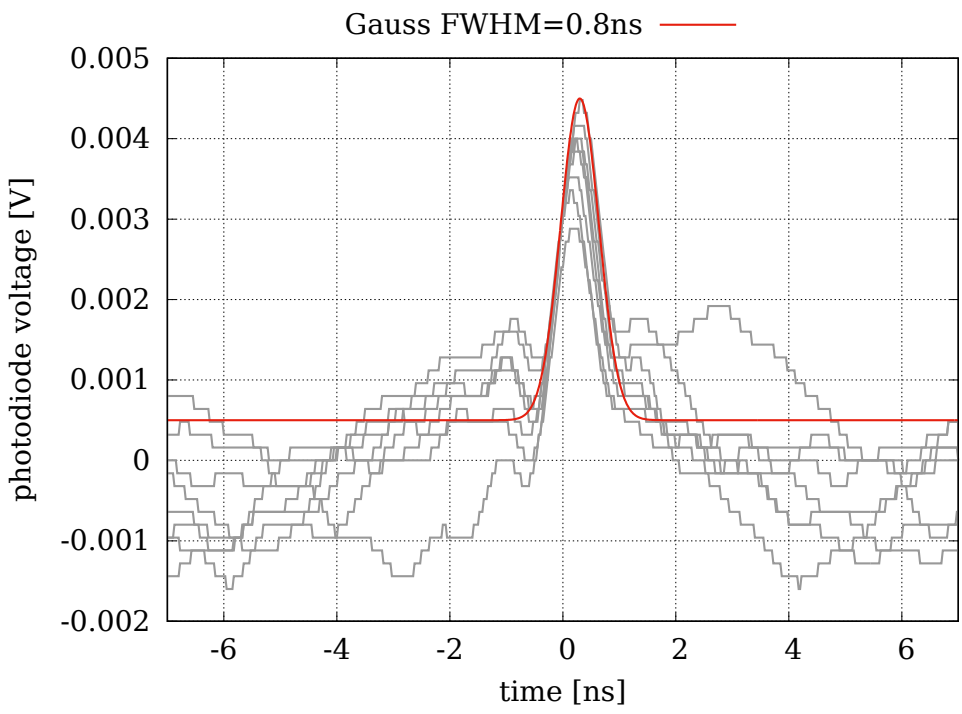

B)

Fig. 3.13: A) Laser pulse duration measured with a high speed photodiode. Percentage refers to the approximate laser energy in terms of $200 \mathrm{~mJ}$. B) Impulse response of the photodiode measured by exposing it to a $5 \mathrm{MHz}$ femtosecond laser (courtesy Dr. Ingo Gregor)

Cameras - With the FASTCAM-APX RS (model 250K) it was possible to record with a minimum shutter time of $1 \mu \mathrm{s}$ and frame rates of $21 \mathrm{kfps}$ at $384 \times 272$ pixels, $100 \mathrm{kfps}$ at $244 \times 222$ pixels and up to $400 \mathrm{kfps}$ at $112 \times 58$ pixels. More than 1000 images per record would be possible, but only up to 60 were necessary.

The Imacon 468 camera has the capability to record up to $100 \mathrm{Mfps}$ at $385 \times 575$ pixels. However, only 8 images in total are captured for any time resolution. Each of the 8 images can be set arbitrarily in start time, exposure time and voltage gain.

The camera objective - used is the K2 Infinity long distance microscope (Infinity-USA 2016) with the CF- 2 and CF-4 modules, allowing for resolutions down to $4 \mu \mathrm{m} /$ pixel and about 
$2 \mu \mathrm{m} /$ pixel respectively, with large working distances of $200 \mathrm{~mm}$ down to $54 \mathrm{~mm}$.

Other measurement tools - The high-speed photodiode SV2-FC from Thorlabs was used as a main tool to, for instance, characterize the light output of the illumination devices (Sec. C.1), to measure the duration of the laser pulse or trigger the camera. Its response spectrum is given in Sec. C.1 and the impulse response is given in Fig. 3.13.

The laser intensity profiles were measured with the Metrolux profiler and Raylux Software.

Illumination - Illumination for high speed photography is always an issue. The predecessors of the lab mostly used the nostalgic photo flash Mecablitz 60 CT-4 using a manufactured $5 \mathrm{~V}$ TTL-pulse trigger. This flash broke in the early stages of this research and Metz nowadays does not produce such powerful flashes anymore ${ }^{1}$, probably because camera sensors have become much more sensitive. The necessity of the situation led to the quest for a proper illumination device and also to the ray-tracing overlay method (Sec. 3.2) as a positive side effect.

To summarize the outcome of the illumination research:

\section{Criteria 3.3. Illumination sources}

1. For kiloframes per second recording frequencies, overdriven high-power LEDs can be used with pulse durations of $\approx 1 \mu$ s, synchronized with the camera.

2. For Megaframes per second the LED solution is still 3 orders of magnitude too less light and the only choice is xenon flash tubes of minimum $30 \mathrm{~J}$ light emission in combination with reflector and Fresnel lens. The light emission duration is in the range $500 \mu \mathrm{s}$ to $8 \mathrm{~ms}$, depending on output energy. As a thumb rule, xenon flash tubes emit $1 \mathrm{~J}$ of light per millimeter tube length at maximum power.

3. Arc discharge lamps with less than $1 \mu$ s emission duration are less practical and were not investigated thoroughly. Quick trials with the Kraftblitz of the lab were done revealing no more light gain but more setup effort.

Concerning point 1, a warm-white $100 \mathrm{~W}$ LED (Chanzon) was mounted onto a cooling block. For the setup shown in this thesis, the first version of the device was still in use, operating with pulses of $\approx 1 \mu \mathrm{s}$ FWHM duration at driving voltage of $40 \mathrm{~V}$. Nominal voltage for constant illumination is stated as between $20 \mathrm{~V}$ and $24 \mathrm{~V}$. The circuit diagram (Bahl and Schönekeß 2020) for the driving is given in Fig. C.7.

The final version of LED illumination device comprises 2 LED chips, manually variable pulse duration, capability of overdriving up to $200 \mathrm{~V}$ and built-in AND circuit for TTL trigger logic.

The following findings were measured ${ }^{2}$ : The LEDs were found to be thermally endangered for pulse durations $\gtrsim 3 \mu$ s or pulse repetition periods in the order of two times the pulse duration. However, the system was quite complex, because long term illumination drains the power from the capacitors and the LED is driven by the power supply which usually cannot provide enough current at high voltages ( $200 \mathrm{~V}$ at $40 \mathrm{~A}$ needed approximately). Limiting voltage for the red LEDs is estimated to be between $180 \mathrm{~V}$ and $200 \mathrm{~V}$ at $1 \mu$ s pulse duration and stable driving circuit.

\footnotetext{
${ }^{1}$ oral communication with sales manager

${ }^{2}$ in cooperation with J. Eisener
} 
The Xenon flashes used were the i) Mettle MT-600DR emitting about $200 \mathrm{~J}$ of light over about $8 \mathrm{~ms}$ by a ring flash tube and ii) an old photo flash (Mecablitz 36CT2) with a straight flash tube of $35 \mathrm{~mm}$ length and Fresnel lens. More details are given in Sec. C.1.

It is found that unexpectedly, the smaller Mecablitz 36CT2 is the best choice for illumination at Megaframes per second, because the flash beam is focused by a Fresnel lens. The Mettle flash beam opening angle is still too broad, even when the reflector shield is attached. Furthermore, the geometry and non-diffuseness of the ring flash tube makes post processing of the data gained a tedious, if not impossible task.

\subsubsection{Setup 1: Large cuvette setup - kfps range}

Setup 1 (Fig. 3.14) comprises a large cuvette $(10 \mathrm{~cm} \times 10 \mathrm{~cm} \times 30 \mathrm{~cm})$ filled with filtered, deionised and de-gassed water. Inside the cuvette a polished aluminium, $90^{\circ}$ off-axis parabolic mirror (Edmund optics \#37-307) with focal length $101.60 \mathrm{~mm}$ and 2 " diameter is mounted. The intention was to create a point focus with high numerical aperture $(\mathrm{NA}=0.32)$. The high speed camera Photron APX-RS is used at recording frequencies of $100 \mathrm{kfps}$ to $400 \mathrm{kfps}$. Attached is the K2 infinity long distance microscope with the CF-2 objective, resulting in approximately $4 \mu \mathrm{m} /$ pixel. The CF-4 objective could not be applied because of the necessary long focal distance between cuvette wall and bubble. Illumination was performed with the overdriven $100 \mathrm{~W}$ LED. A circuit was built (see Fig. C.7) to discharge high voltage capacitors over the LED for $1 \mu \mathrm{s}$. Later it was found that this kind of illumination is best for applications where the minimum shutter time of the camera is too long to capture sudden bubble phenomena. For high speed imaging, especially for Megaframes per second, overdriven LEDs are too dim by a factor of 1000 compared to xenon flash lights. Also, the trigger circuit becomes increasingly complex since the camera and the LED must be synchronized, but only for recording. The LED must not fire when not recording, because the capacitors last for only 1000 shots. The LED device was improved until an overdriving factor of 6 (see Sec. C.1), however the light yield was only $26 \mu \mathrm{J}$ per pulse.

In this setup the first version of LED illumination was used. The color was warm white, because the camera system including objective and notch filter was found to be most sensitive in the red regime. Warm white spectra are generated with UV-LEDs and a fluorescence screen glued on top. This was also favourable because of the diffusing properties of the screen. The notch filter is supposed to filter out any laser stray light. 
a)

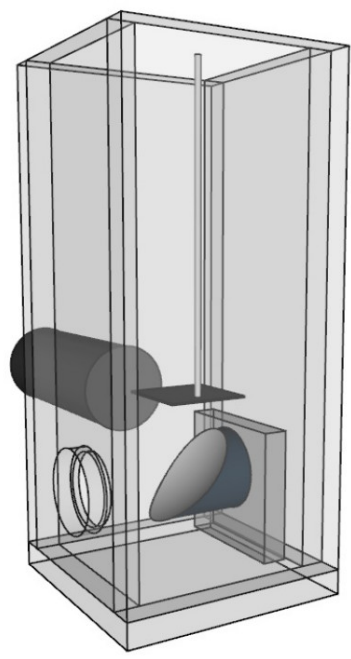

b)

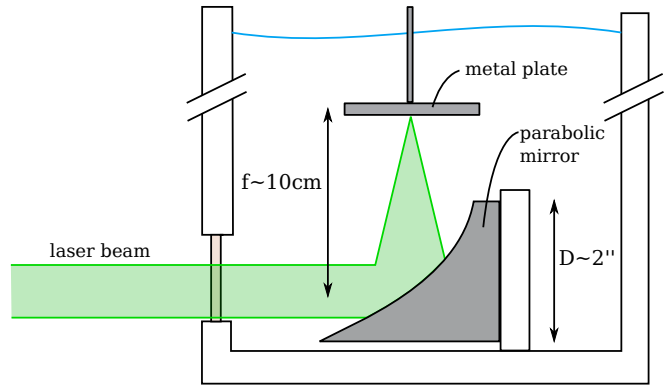

c)

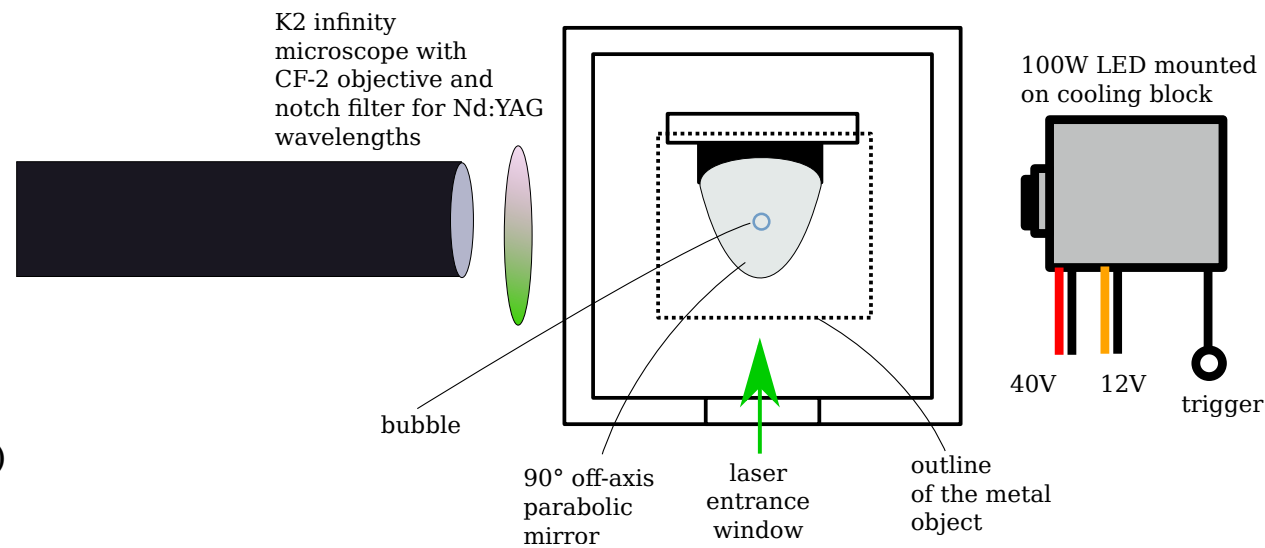

Fig. 3.14: Sketch of setup 1. a) 3D CAD representation of the objective and cuvette with parabolic mirror, mirror holder and metal object table. Laser entrance window on the left. b) 2D side view with sketched laser path. The parabolic mirror both reflects in $90^{\circ}$ direction and focuses. Numerical aperture is 0.32 . c) $2 \mathrm{D}$ top view including the illumination LED (pulsed, overdriven $100 \mathrm{~W}$ LED)

The trigger circuit is shown in Fig. 3.15. The delay 1 device constantly produces the laser flash lamp TTL trigger pulse at $15 \mathrm{~Hz}$. One of 20 of the pulses is extracted and can be "let through" by a hardware button to the next delay generator 2 . This delay generator produces a pulse of zero delay for an optional flash (assuming its warm-up time to be around $160 \mu \mathrm{s}$ ) and a $600 \mu$ s long pulse with $160 \mu$ s delay for the Q-Switch of the laser and camera record trigger. This pulse is also put into one of the two inputs of an AND-logic for the LED flash trigger. The camera itself has an output with a high-signal when the shutter is open. This signal is put into delay 3 where the pulse is delayed by $8.1 \mu$ s for $100 \mathrm{kfps}$ record speed. The result is fed into the second input of the AND-logic, thus opening the trigger for the LED. This accounts for the delays in the LED circuit, as well as skipping illumination of the first record frame to be able to record the plasma breakdown, too. Lastly, the photodiode helps to adjust the triggering. 


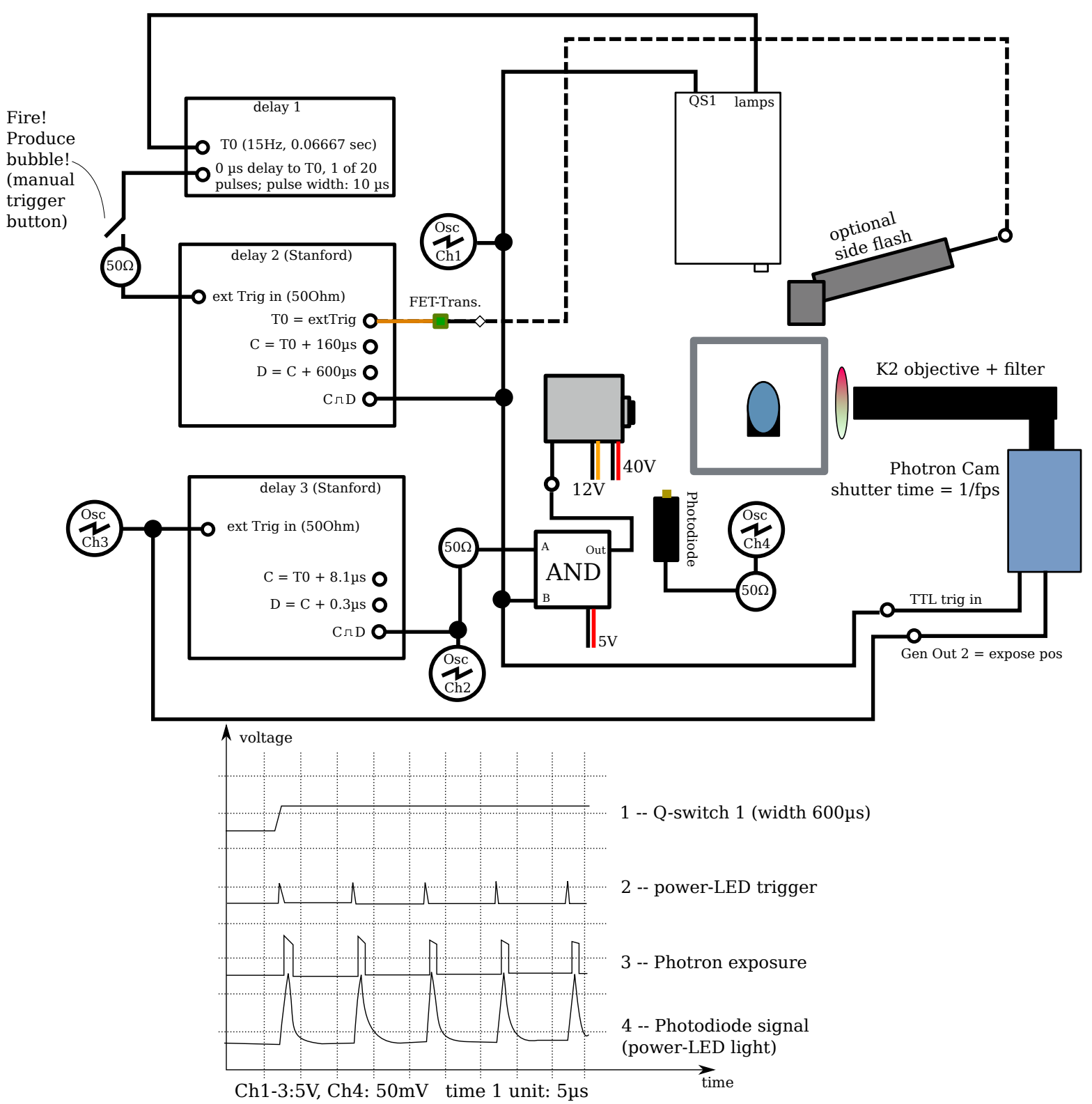

Fig. 3.15: Setup 1: Block circuit diagram and schematic oscilloscope diagram of the synchronization setup of laser, illumination and camera

\subsubsection{Setup 2: Small cuvette setup - close to Mfps range}

This setup (Fig. 3.16) is designed for ultra-high-speed (mega frames per second) photography ${ }^{3}$. The camera used is the IMACON 468 capable of recording 8 images with maximum $100 \mathrm{Mfps}$ time resolution. Attached to it is the $\mathrm{K} 2$ long distance microscope with the $90^{\circ}$ mirror element put to close to the cuvette-side of the microscope. Two cuvette sizes are used, both much smaller than the one in setup 1 in Sec. 3.3.1: Cuvette A has dimensions $1 \mathrm{~cm} \times 1 \mathrm{~cm} \times 4 \mathrm{~cm}$ (width, depth, height) and is made of a plastic but with glass window for laser entrance and cuvette $B$ has dimensions $5 \mathrm{~cm} \times 1 \mathrm{~cm} \times 4 \mathrm{~cm}$ and a wall thickness of $2 \mathrm{~mm}$.

The light from the Nd:YAG laser is focused by a lens with a short focal length $(35 \mathrm{~mm})$ di-

\footnotetext{
${ }^{3}$ in cooperation with J. Rosselló
} 
rectly onto the surface of a planar glass object, located at the rear side of cuvette A and in the middle of cuvette $\mathrm{B}$.

a)
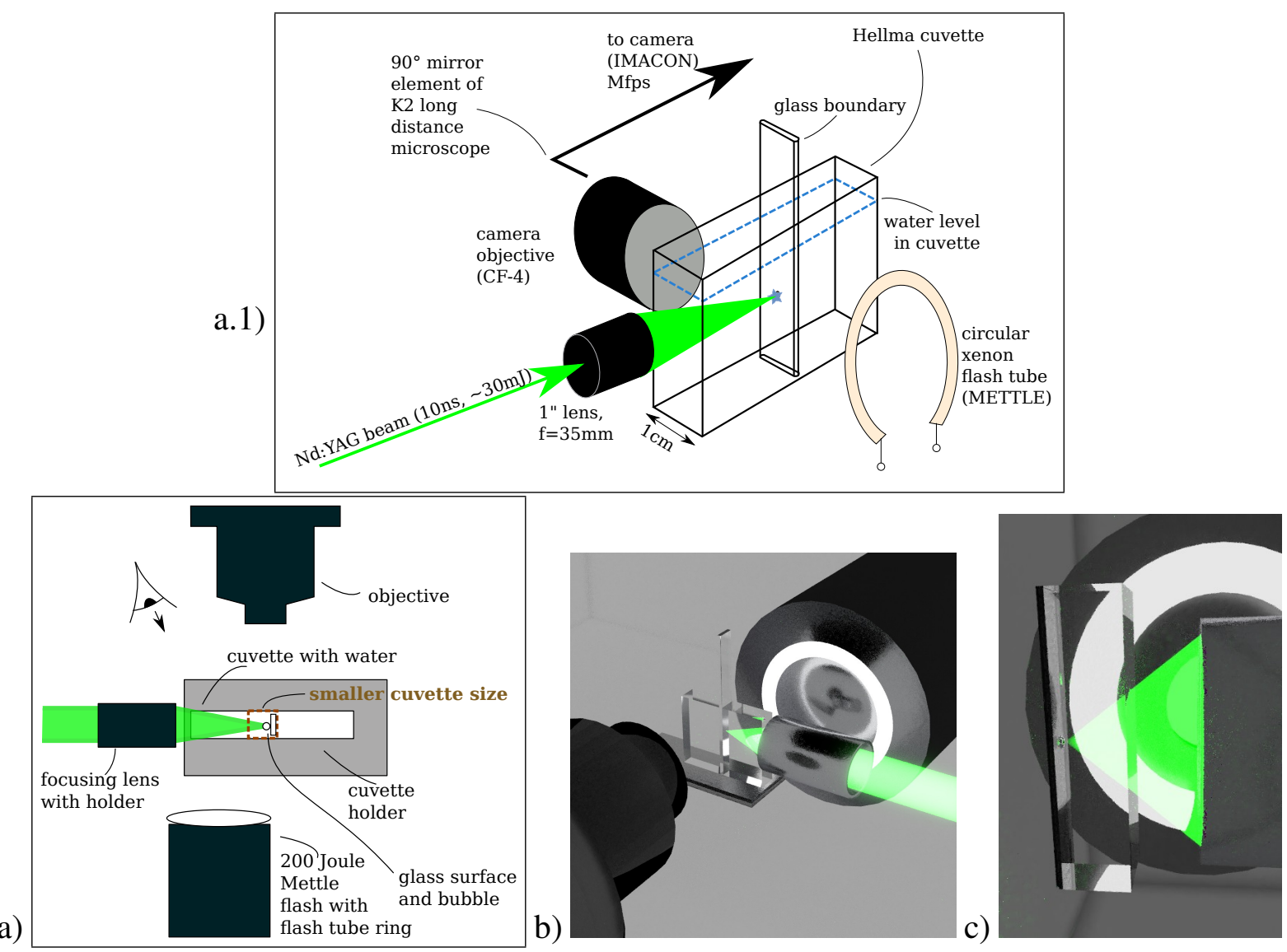

Fig. 3.16: Sketch (a and a.1) and blender-ray-traced representation (b),(c) of the experimental setup 2. The bubble is generated with a nanosecond pulse of a frequency doubled Nd:YAG laser with pulse energy of about $30 \mathrm{~mJ}$. The bubble is produced on a glass surface put vertically into the cuvette. Illumination is done by the Mettle flash with ring xenon tube.

\subsubsection{Setup 3: Small cuvette setup - Mfps range}

This setup was designed to investigate the bubbles on a rigid cylinder, but can be extended to any investigations with a small, or transparent object. The setup 2 is modified by omitting the ring flash tube and only using the Mecablitz, furthermore combining both the Imacon and Photron high speed cameras and adding a continuous wave He-Ne laser beaming through the bubble site hitting the photodiode for trigger usage. The Photron camera is supposed to capture the whole bubble life time at a lower frame rate and larger frame size. The Imacon is used for the Megaframes per second frame rate and high zoom into the bubble site. This posed again illumination difficulties because the Photron camera has a much longer shutter time and a different viewing angle. The small rectangular mirror, however, simply compensates the difficulties by reflecting only light from outer part of the flash beam towards the Photron objective.

The path of the He-Ne-laser beam goes through the bubble site, various mirrors and pinholes onto the photodiode, that is shaded against any stray light from the flash. Depending on the size of the bubble, the light is more or less deflected, yielding a photodiode signal that is highest 
when the bubble is absent. It turned out that the intensity of the He-Ne laser has a strong highfrequency modulation, which is why the signal had to be low-pass filtered by an analog device before detection by the oscilloscope. The oscilloscope is equipped with a hardware trigger TTL output. This output is set to give a TTL-high when the photodiode voltage crosses a certain level with positive slope. The TTL-high then triggers the Imacon camera. This way the trigger accuracy could be increased to approximately $1 \mu$ s precision onto certain bubble dynamics parts. This is enough to gain $10 \mathrm{Mfps}$ resolution with one single measurement skipping weeks of unlucky shots.

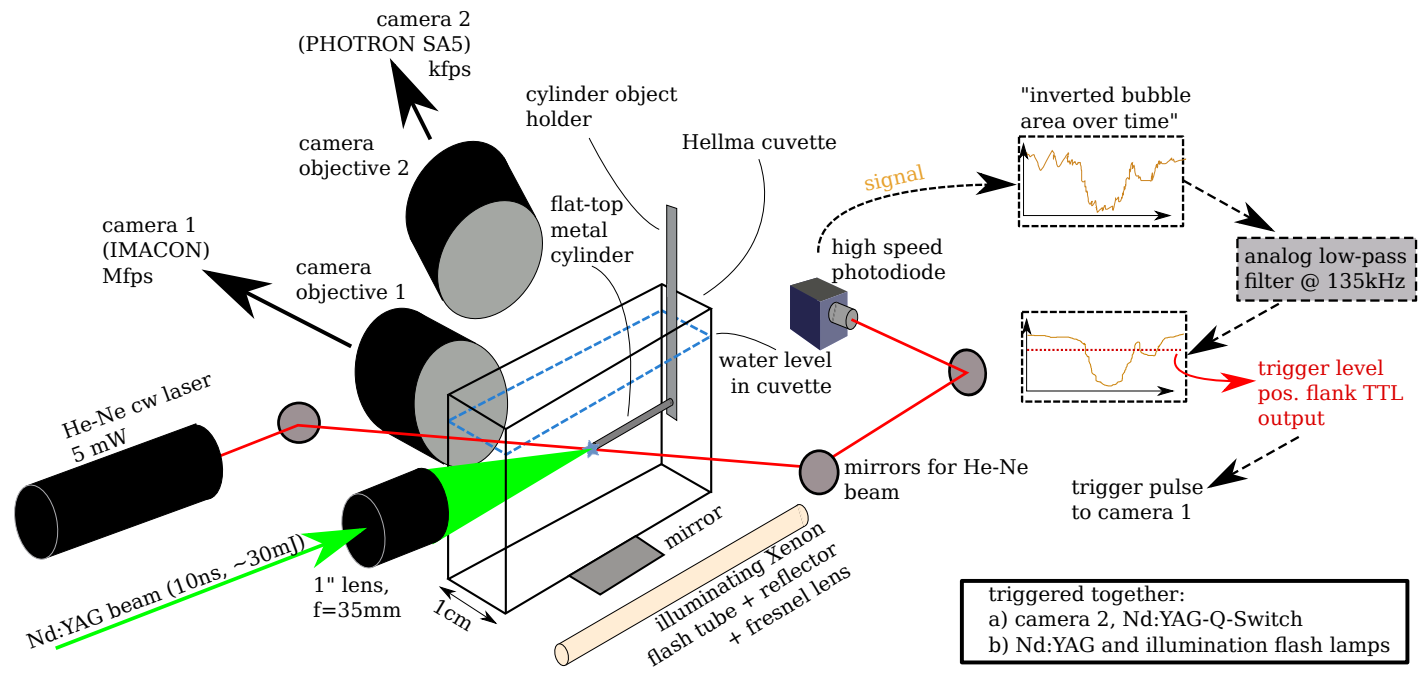

Fig. 3.17: Sketch of the setup 3 used to investigate the bubbles on a rigid cylinder. The He-Ne-laser allows for triggering onto bubble dynamics parts with approximately $1 \mu$ s precision. The xenon flash tube refers to the Mecablitz already introduced in the previous setups. 


\section{Bubble dynamics at the planar, solid boundary}

\subsection{Vortex generation and the Lagrangian Inkmap}

Reuter et al. (2017a) developed an experimental $\mu$-particle tracking velocimetry method to follow the flow field around a collapsing bubble in the vicinity of a solid boundary. It was found that the bubble generates a vortex that, depending on $D^{*}$, travels either outwards alongside the solid boundary (wall vortex) or orthogonally away from the solid boundary (free vortex). The authors used a light-sheet generated by a continuous-wave laser to stimulate the velocity tracking particles, thus they got a cross-section of the flow field and assumed its rotational symmetry. Due to the limited time and spatial resolution and the opaqueness of the bubble in the experiment, these data nearly provoke to be interpolated by numerical simulations, to get a clear understanding of how the vortices are formed.

Figure 4.1 shows the classification of the directions. The quantity $\gamma_{d}$ is defined as the ratio of the distance of the bubble center from the solid boundary $d$ to the radius of the bubble $R$. As discussed in Sec. 3.1.1, to be precise with the definition is crucial. In oral communication with the first author, for the work Reuter et al. (2017a) the folloing definition for $\gamma_{d}$ could be achieved:

$$
\gamma_{d}=\frac{d(t \approx 4 \mu \mathrm{s})}{R\left(t=t\left(V_{\max }\right)\right)}
$$

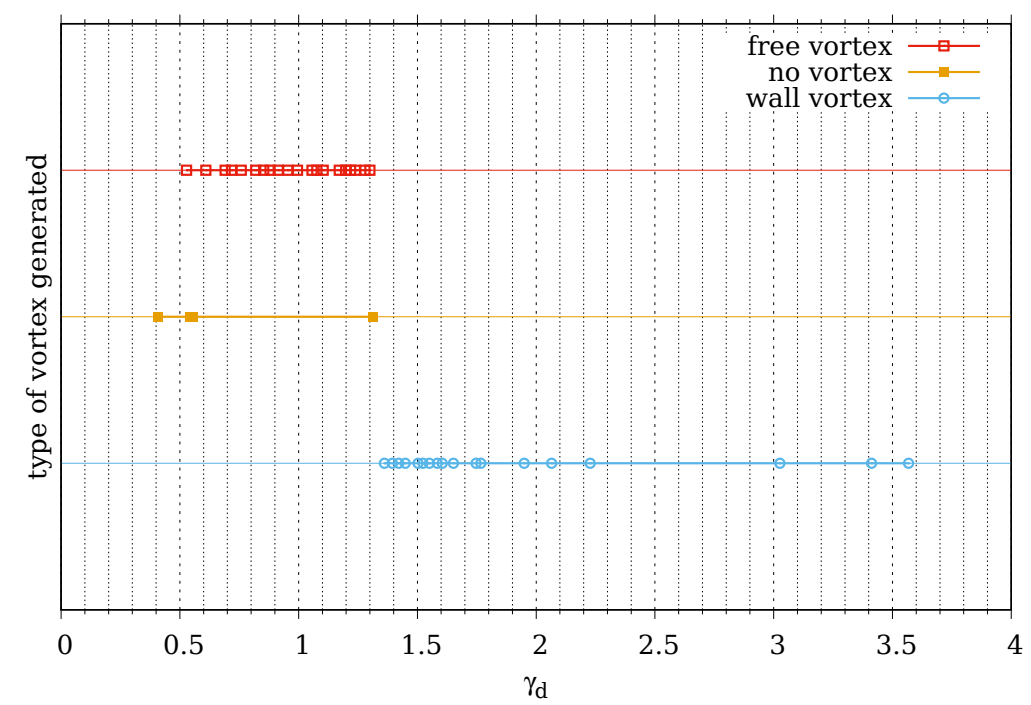

Fig. 4.1: Classification of vortex direction, data taken from Reuter et al. (2017a).

The $4 \mu$ s time is an approximate value because the distance was taken from the first image of the record, which was done with $250 \mathrm{kfps}$. The radius at maximum expansion $R\left(t=t\left(V_{\max }\right)\right)$ was 
said to be extracted from the bubble volume. Now depending on which conversion from $D^{*}$ to $\gamma_{d}$ is taken, the simulations represent the data in Fig. 4.1 very well or are off in the region below $\gamma_{d}=0.6$. In that region, the pure numerical results can provoke discussion by themselves. That is why, in Sec. 4.1.1 first the perfectly fitting results are shown and afterwards in Sec. 4.1.2 they are discussed. However, the general result of both the experimental work and this thesis stays untouched, namely that the vortex flow changes direction at about $\gamma_{d} \approx D^{*} \approx 1.3$. The discussion focuses more on the lower values of the dimensionless distance.

\subsubsection{Results for different normalized bubble distances $D^{*}$}

The simulations shown here are carried out with the axisymmetric, polar Mesh 3.1.2.a that was tested in Sec. B.1.3. The algorithm 1 of Tab. 3.1 is applied for the initial data, following the idea to use a gas cylinder as initial bubble that fits perfectly into the mesh, aligned with the edges of the cells, in order to avoid discretisation errors during setup. The volume of the cylinder is then re-evaluated and the gas pressure $p_{g}$ is adjusted according to the adiabatic law. The assumption behind adjusting the pressure this way instead of keeping the bubble energy constant is that obeying the adiabatic law for a fixed $R_{n}$ results into the same $R_{\text {equiv. }}(t)$-curve. To give this assumption a concrete base, the very same simulation that is going to be made in this section has been preformed in unbounded liquid, too, with both the axisymmetric mesh and also the spherical mesh. The double collapse-time is then compared to the reference solutions of Fig. 3.8, given in Fig. 4.2.

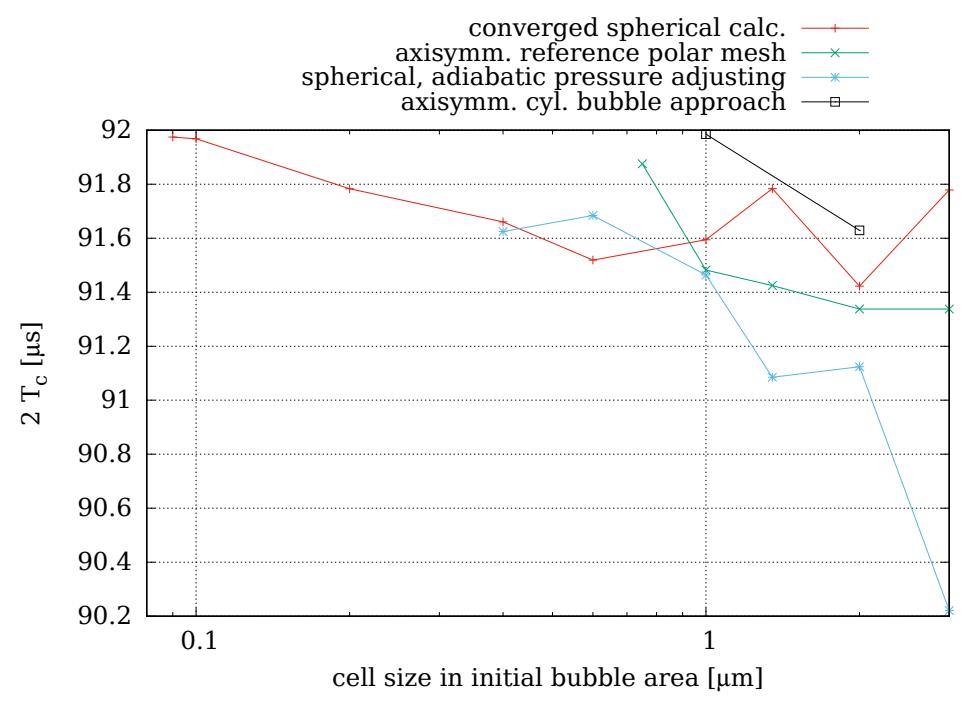

Fig. 4.2: Simulations of the mesh parameters and initial data for this section, but in unbounded liquid. Compared to the reference solutions of Fig. 3.8.

It is seen that the cylinder approach is the only approach so far, reaching the convergence value obtained with the spherical reference solution, already at a resolution of $1 \mu \mathrm{m}$. The spherical calculation with adiabatic pressure adjustment also suggests that there is no clear disadvantage in either of the two pressure adjustment algorithms. To show that the cylindrical shape vanishes quickly, aligning well with the spherical shape, the contour plots of the bubble interface for the cylinder approach and the spherical approach, used in the discussion Sec. 4.1.2 later, are given in Fig. 4.3.

The geometric parameters for the investigation were chosen as given in Tab. 4.1. 


\begin{tabular}{|lllllcl|}
$\Delta x_{\min }$ & $\mathrm{Xi}$ & $\mathrm{Xii}$ & $\mathrm{X}$ & $\mathrm{XF}$ & grading factor $g_{f}$ & initial data \\
\hline \hline $2 \mu \mathrm{m}$ & $80 \mu \mathrm{m}$ & $1.2 \cdot \sqrt{2} \cdot \mathrm{Xi}$ & $1.2 \cdot R_{\max }$ & $80 R_{\max }$ & 5.25 & $\begin{array}{l}\text { cylinder, } \\
p V^{\gamma}=\text { const. } \\
\end{array}$ \\
& & & & & \\
& & & & & algorithm 1 \\
\hline
\end{tabular}

Table 4.1: Setup parameters of the simulations of the vortices of a collapsing bubble close to a solid boundary.

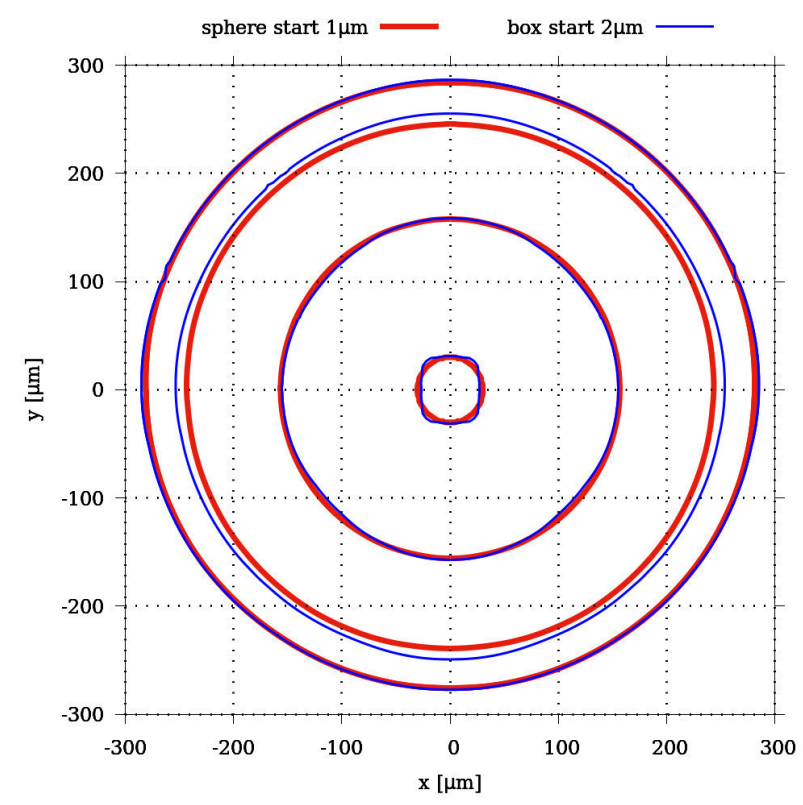

Fig. 4.3: Contour lines of the bubble interface for the cylinder approach (algorithm 1, box start, blue lines, equivalent starting radius of $19.73 \mu \mathrm{m}$ ) and the sphere approach (algorithm 2, sphere start, red lines, equivalent radius of $20.06 \mu \mathrm{m}$ ). Times: $10^{-10} \mathrm{~s}, 1 \mu \mathrm{s}, 4 \mu \mathrm{s}, 6 \mu \mathrm{s}$

Since the vortices develop very late in the bubble process, the calculation needs to run for a long time for each value of $\gamma_{d}$. Therefore, a script was written to supervise the calculation and change the time stepping when the fast bubble dynamics is over. The changes made are given in Tab. 4.2:

\begin{tabular}{l|lllll} 
time & maxCo & maxAlphaCo & maxAcCo & $\begin{array}{l}\operatorname{maxAcCo}=1 \\
\text { for } R / R_{n}<\end{array}$ & maxDeltaT \\
\hline $0 \mathrm{~s}-120 \mu \mathrm{s}$ & 0.2 & 0.2 & 8 & 0.3 & $5 \cdot 10^{-8} \mathrm{~s}$ \\
$120 \mu \mathrm{s}-200 \mu \mathrm{s}$ & 0.2 & 0.2 & 30 & 0.01 & $5 \cdot 10^{-7} \mathrm{~s}$ \\
$200 \mu \mathrm{s}-$ end & 0.4 & 0.4 & 100 & 0.01 & $5 \cdot 10^{-7} \mathrm{~s}$
\end{tabular}

Table 4.2: Time stepping of the calculations in Sec. 4.1.1 to save calculation time. maxCo: maximum flow Courant number, maxAlphaCo: maximum Courant number for the interface, $\operatorname{maxAcC}$ : maximum acoustic Courant number, maxDeltaT: maxmimum size of the time step

The Lagrangian ink map is calculated as described in Sec. 2.4.2 and colored in a way that produces good contrast between the liquid layers. The initial data for the passive scalar $\Gamma(\mathbf{x}, t)$ that is advected with the flow is a clipped linear ramp in direction of the axis of symmetry 
(y-direction):

$$
\Gamma(\mathbf{x}, t=0)= \begin{cases}\frac{y}{1.5 R_{\max }} & \forall y<1.5 R_{\max } \\ 1 & \forall y \geq 1.5 R_{\max }\end{cases}
$$

The values of $D^{*}$ investigated here are:

$$
D^{*} \in[0.2,0.4,0.6,0.8,1.0,1.2,1.4,1.6,1.8]
$$

In order to get an idea of the bubble volume over time compared to the time of vortex generation, the equivalent radius over time is given for $D^{*}=[0.2,1.8]$ in Fig. 4.4. It is seen that with the applied bubble model, the bubble closer to the solid boundary exhibits more rebound oscillations than the bubble further away from the solid boundary.

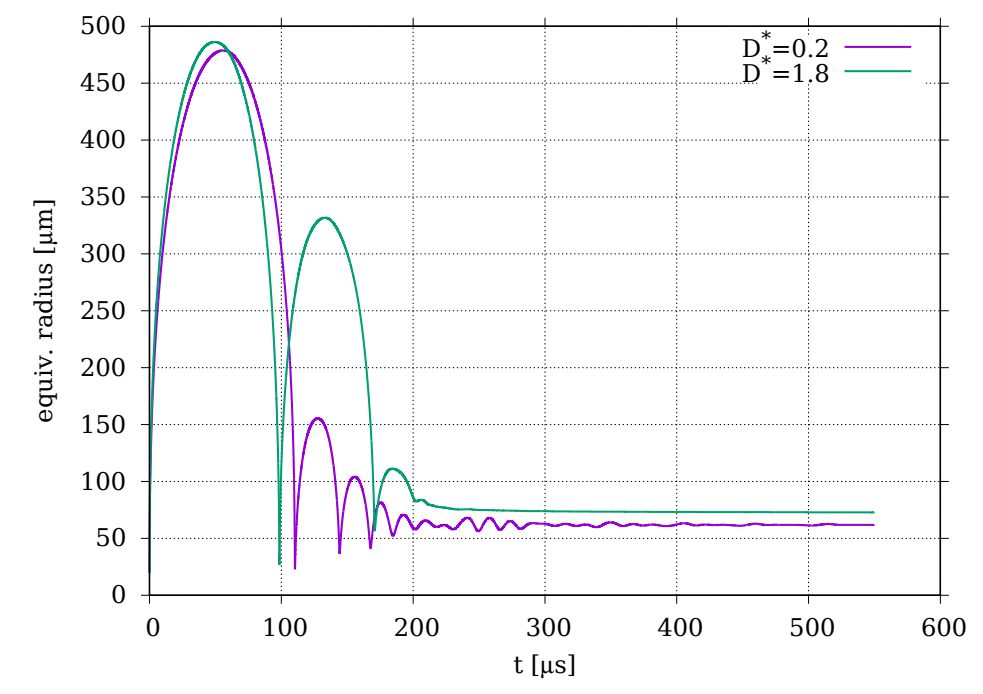

Fig. 4.4: Equivalent radius over time for $D^{*}=[0.2,1.8]$ with the cylinder approach.

The results for the Lagrangian inkmap figures are given in Figs. 4.5 to 4.8. One has to keep in mind that the spatial resolution is highest in the area of the initial bubble, thus vortices far from that region become under-resolved regarding the winding of liquid layers. 


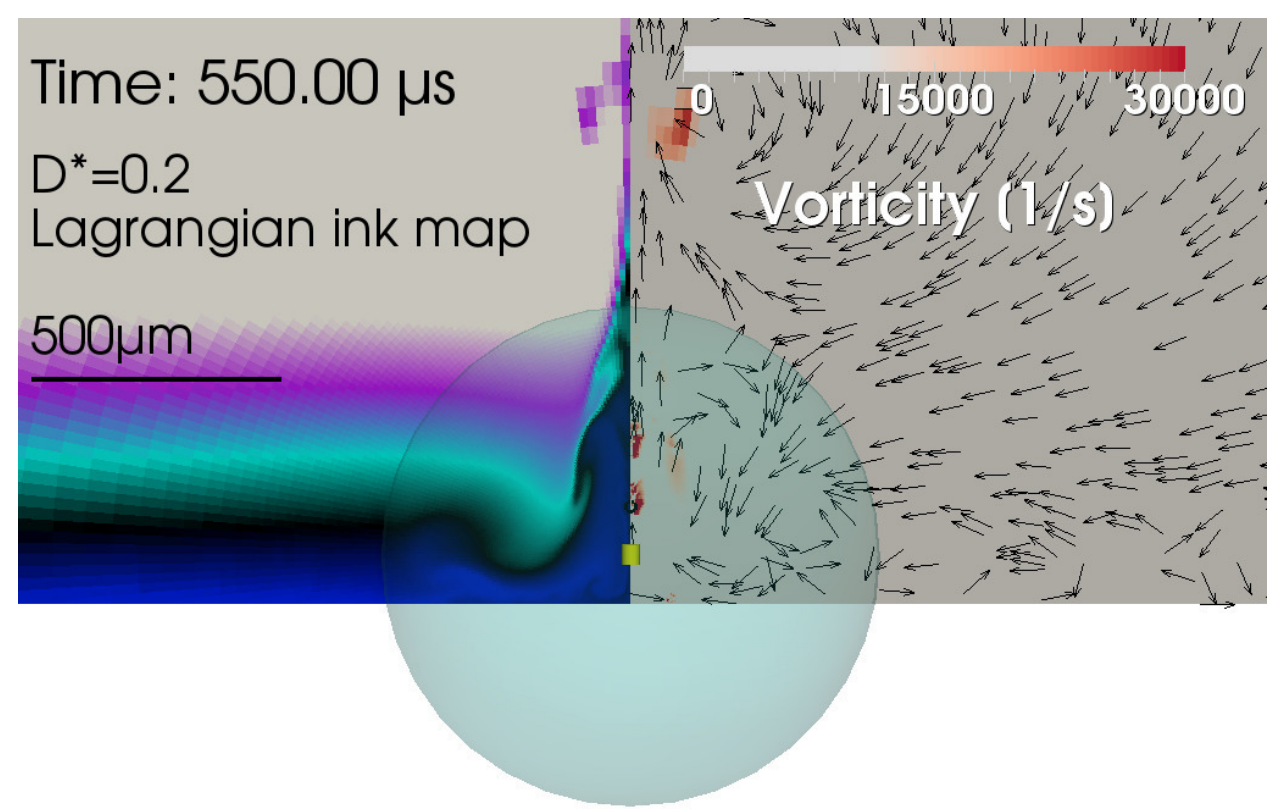

Fig. 4.5: Free vortex generated by the $D^{*}=0.2$ bubble. Left: Lagrangian-ink map. Right: vorticity $\nabla \times \mathrm{U}$ in $\mathrm{s}^{-1}$ including arrows for flow direction. The yellow cylinder denotes the initial bubble size and position, whereas the larger, transparent sphere denotes the maximum volume the theoretical bubble would attain in an unbounded liquid. The black contour lines, mostly seen at the axis of symmetry, mark the areas of remnant gas. Here for $D^{*}=0.2$ it is seen that liquid of the upper part (marked with colors pink to turquoise) is already pushed upwards out of sight near the axis of symmetry. The main vortex rotation is clockwise.
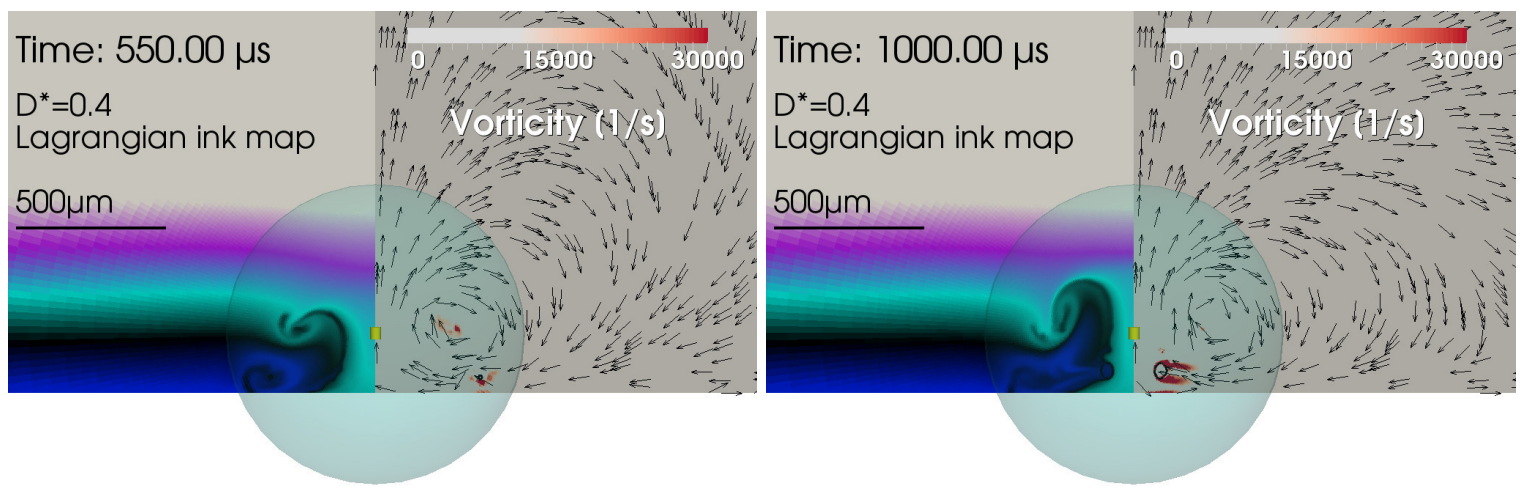

Fig. 4.6: No moving or spreading vortex generated by the $D^{*}=0.4$ bubble for a late time and a very late time instant to show the almost positional stability of the vortex. More details see text and Fig. 4.5.

Figure 4.6 shows a bubble which within a long time won't shed a vortex. The vortex rotation is seen in the flow field and the direction (clockwise) indicates a free vortex, but the velocity is too slow. 

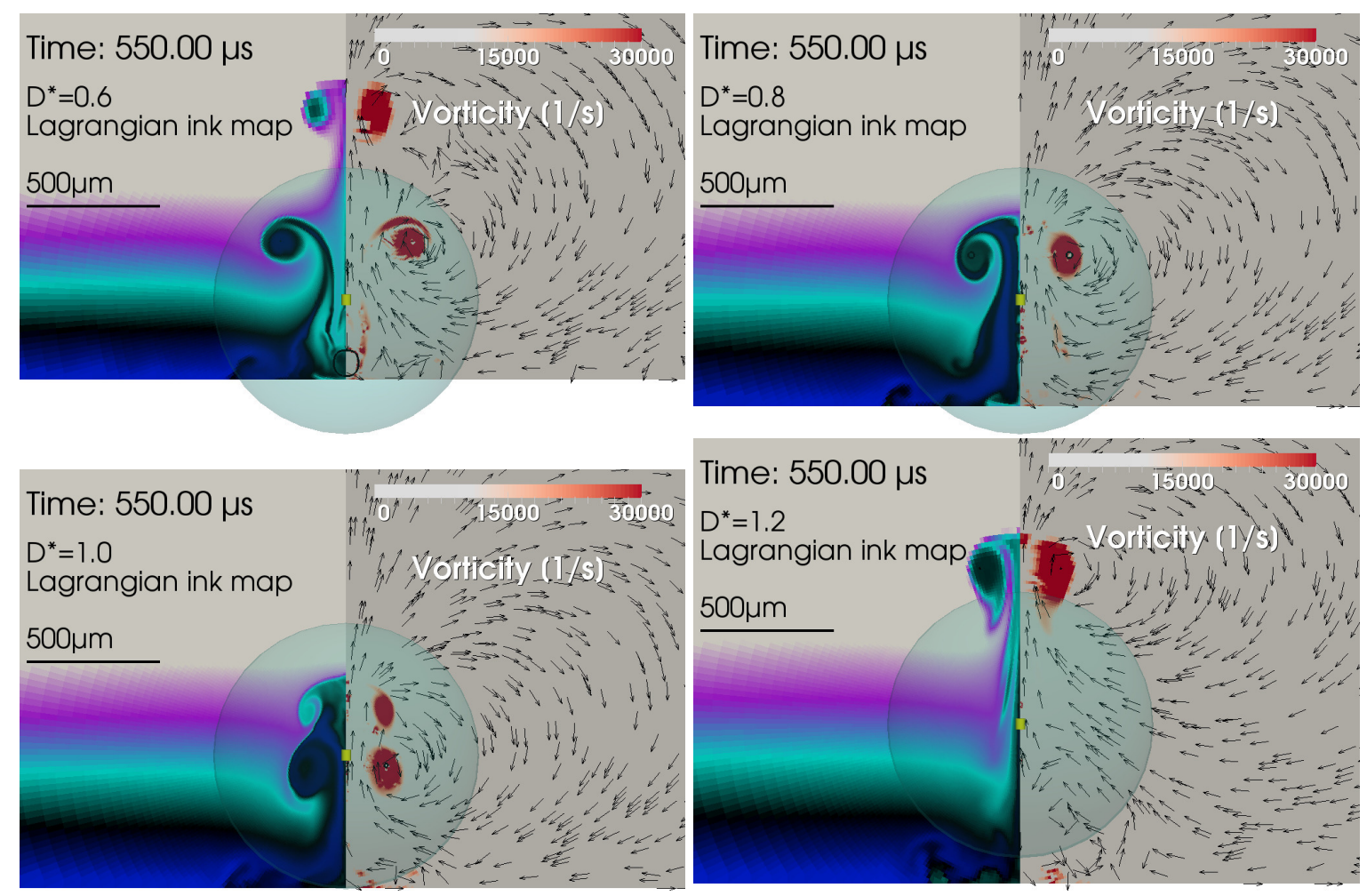

a)
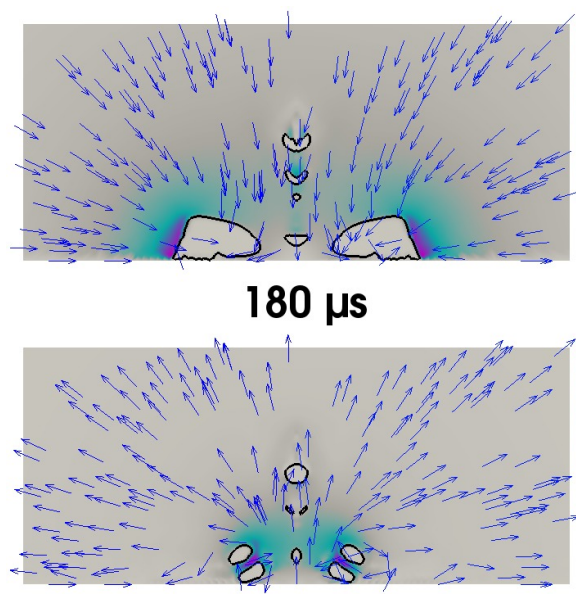

b)
$180 \mu \mathrm{s}$

21.8

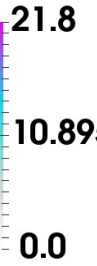

190 us

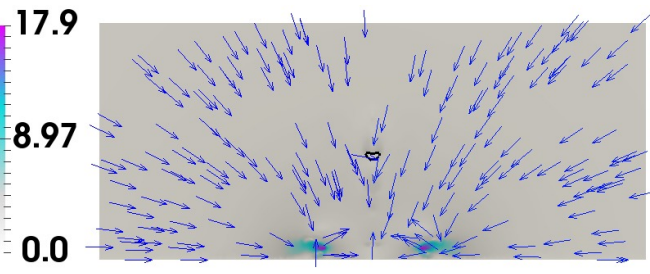

$185 \mu s$

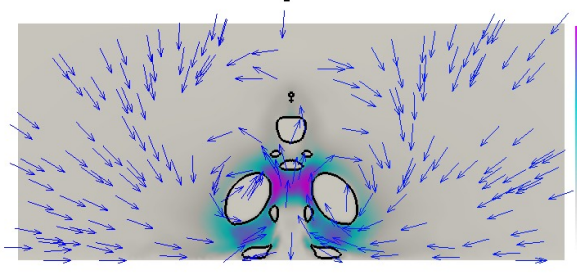

$210 \mu \mathrm{s}$
61.1

30.574

0.0

10.5

5.2567

0.0

Fig. 4.7: a) Free vortex/ free vorticies generated by the $D^{*} \in[0.6, \ldots, 1.2]$ bubbles. More details see text and Fig. 4.5. b) The evolution of the flow field $(\mathrm{m} / \mathrm{s})$ around the bubble for $D^{*}=1.2$ during the second collapse. The inwards, sideways fluid motion at $180 \mu \mathrm{s}$ is responsible for the change of the flow direction at the center axis after bubble minimum volume.

All bubbles at $D^{*} \in[0, \approx 10]$ produce a jet towards the solid boundary, so the main fluid motion is expected to be directed towards the solid boundary. However, for the $D^{*}$-values shown in Fig. 4.7 a, the bubbles generate an axially symmetric vortex that lifts upwards from the solid boundary. The reason is found in the dynamcis of the second bubble collapse. This is depicted in Fig. $4.7 \mathrm{~b}$ for $D^{*}=1.2$ : the bubble torus sitting at the solid boundary collapses fastest from the outer rim, towards the axis $(180 \mu \mathrm{s})$. After the second minimum volume of the 
bubble at about $185 \mu$ s the shockwave inverts the flow direction for a short moment $(190 \mu \mathrm{s})$. The momentum generated by the initial inward flow then produces an upward flow at the axis $(210 \mu \mathrm{s})$.

For the values of $D^{*}$ higher or equal than 1.4 the turning direction of the vortex changes, as well as the motion direction, resulting into a wall vortex (Fig. 4.8). The under-resolved wall vortex (black) can be seen when the position of highest vorticity is compared to the colored ink-map. At least in the simulations, there is also a noticeable part of the top liquid pushed upwards. One supposes a numerical fragment due to the higher resolution region at the position of the initial bubble. However, when looking at Figure 3 in Reuter et al. (2017a), the bubble for $\gamma_{d}=1.42$ exhausts a slow gas remnant upwards, too.

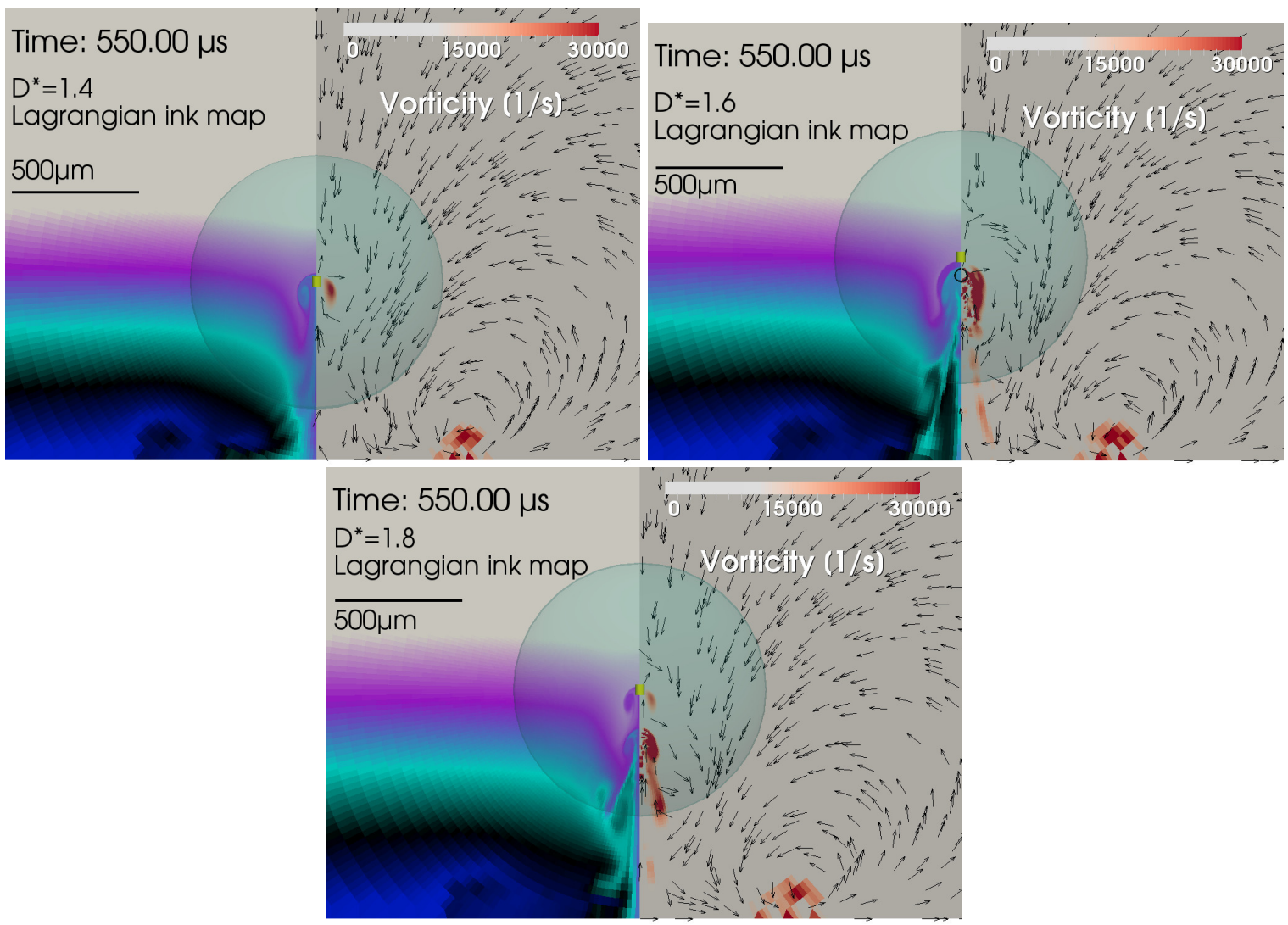

Fig. 4.8: Wall vortices generated by the $D^{*} \in[1.4, \ldots, 1.8]$ bubbles. More details see text and Fig. 4.5.

So the criteria for deciding whether a free vortex, a wall vortex or no vortex/ stationary vortex is created, are as follows:

\section{Criteria 4.1. Vortex classification}

- A free vortex clearly pushes blue or black liquid layers through the purple layer and globally exhibits a clockwise fluid rotation

- A wall vortex clearly pushes liquid from the turquoise layer to the solid boundary and globally exhibits a counter-clockwise fluid rotation

- No vortex/ a stationary vortex is created when after $1000 \mu$ s none of the above points applies. 
When the vortex direction findings of the Figs. 4.5 to 4.8 are put into the diagram 4.1 , considering these criteria and using the simplest $D^{*}$ to $\gamma_{d}$ conversion rule $\gamma_{d}=D_{\text {init }} / R_{\max }\left(t=t\left(V_{\max }\right)\right)$, perfect agreement is found between numerics and experimental data. The result is given in the Fig. 4.9. This perfect agreement is discussed in the next section.
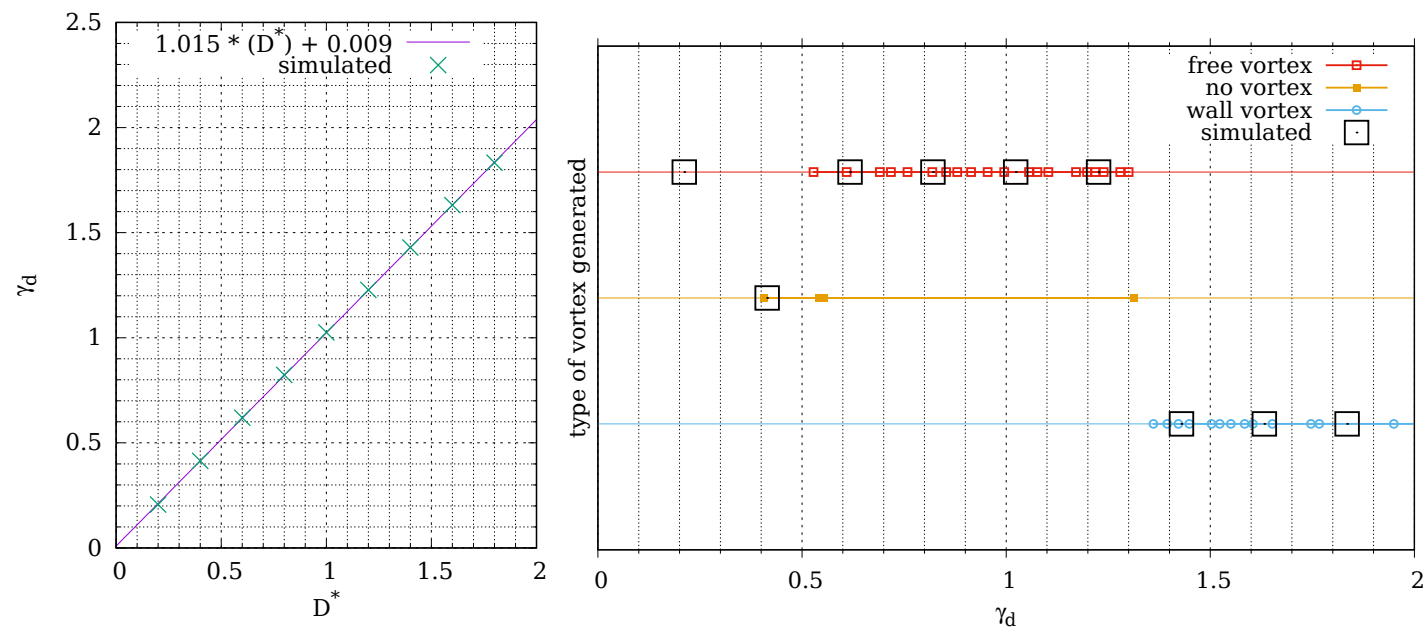

Fig. 4.9: Left: Fit for converting $D^{*} \rightarrow \gamma_{d}$. Right: Comparison of the simulated vortex direction to experiments (Reuter et al. 2017a).

\subsubsection{Discussion of the vortex results}

The simulations in the previous section have been re-made obeying the best practice guidelines for solution convergence (Crit. 3.2 mentioned in Sec. 3.1.4). Special care was taken to reduce the amount of cells while putting the outer boundary from $80 R_{\max }$ to a distance of $100 R_{\max }$. The time interval of the reduction of $R_{n}$, which was coinciding with the time of minimum bubble volume in the previous section ( $90 \mu$ s to $105 \mu$ s), is now put well before the time of minimum volume, namely to $60 \mu$ s to $75 \mu \mathrm{s}$. The basis for the mesh is the axisymmetric, polar Mesh 3.1.2.a that is tested in Sec. B.1.3 and was used in the previous section, too. In the previous section the resolution was $2 \mu \mathrm{m}$, which is now reduced to $1 \mu \mathrm{m}$. The resolution is taken as the name tag for the two kinds of respective parameter sets, which compare as given in Tab. 4.3.

\begin{tabular}{|l||l|l|l|l|c|l|l|} 
& $\mathrm{Xi}$ & $\mathrm{Xii}$ & $\mathrm{X}$ & $\mathrm{XF}$ & grading factor $g_{f}$ & refinement at wall & initial data \\
\hline \hline $\mathbf{2 \mu \mathrm { m } \text { calc. }}$ & $80 \mu \mathrm{m}$ & $1.2 \cdot \sqrt{2} \cdot \mathrm{Xi}$ & $1.2 \cdot R_{\max }$ & $80 R_{\max }$ & 5.25 & no & $\begin{array}{l}\text { cylinder, } \\
p V^{\gamma}=\text { const. } \\
\text { algorithm } 1\end{array}$ \\
\hline $\mathbf{1 ~ \mu \mathrm { m } \text { calc. }}$ & $50 \mu \mathrm{m}$ & $2 \cdot \mathrm{Xi}$ & $1.05 \cdot R_{\max }$ & $100 R_{\max }$ & 5.2 & $\begin{array}{l}\text { yes, } \\
D^{*}=0.4: \text { both } \\
D^{*}=0.42: \text { no }\end{array}$ & $\begin{array}{l}\text { sphere, } E=\text { const., } \\
\text { adapt } R_{n} \\
\text { algorithm 2 }\end{array}$
\end{tabular}

Table 4.3: Setup and initial data of the two approaches to calculate the vortices of a collapsing bubble close to a solid boundary.

The term refinement at wall refers to whether the cell size was halfed in a rectangular region at the wall $\left(x \in\left[0 ; 0.7875 D_{\text {init }}\right], y \in\left[-D_{\text {init }} ;-0.875 D_{\text {init }}\right]\right.$, with $y=-D_{\text {init }}$ at the solid boundary). This was done in order to better resolve wall shear flows. It turned out, however, that parasitic currents occur, when the bubble interface is in that region. This is the case at times of maximum expansion for $D^{*} \lesssim 0.4$, as well as during rebound in all the studied values of $D^{*}$. Therefore, in this dataset, the data point $D^{*}=0.2$ is omitted due to spurious currents. For 
$D^{*}=0.4$ and 0.42 the simulations were repeated without the refinement at the wall.

Cylinder and sphere in initial data in Tab. 4.3 refer to the the shape and algorithm the bubble initial data is set up. The $2 \mu \mathrm{m}$-approach was set up with algorithm 1 of Tab. 3.1, while the $1 \mu \mathrm{m}$ approach makes use of algorithm 2.

The time stepping procedure is the same as in the previous Sec. 4.1.1.

It is left to evaluate the influence of the choice of time interval for the reduction of $R_{n}$, before going on with the $1 \mu \mathrm{m}$ calculations. A small convergence study with the same simulation as in the previous Sec. 4.1.1, including the boundary distance at $80 R_{\max }$, but in unbounded liquid was carried out with both $R_{n}$-reduction intervals ( $60 \mu$ s to $75 \mu$ s and $90 \mu$ s to $\left.105 \mu \mathrm{s}\right)$. The resulting values for $2 T_{c}$ are given in Fig. 4.10 compared to the reference solutions of Fig. 3.8.

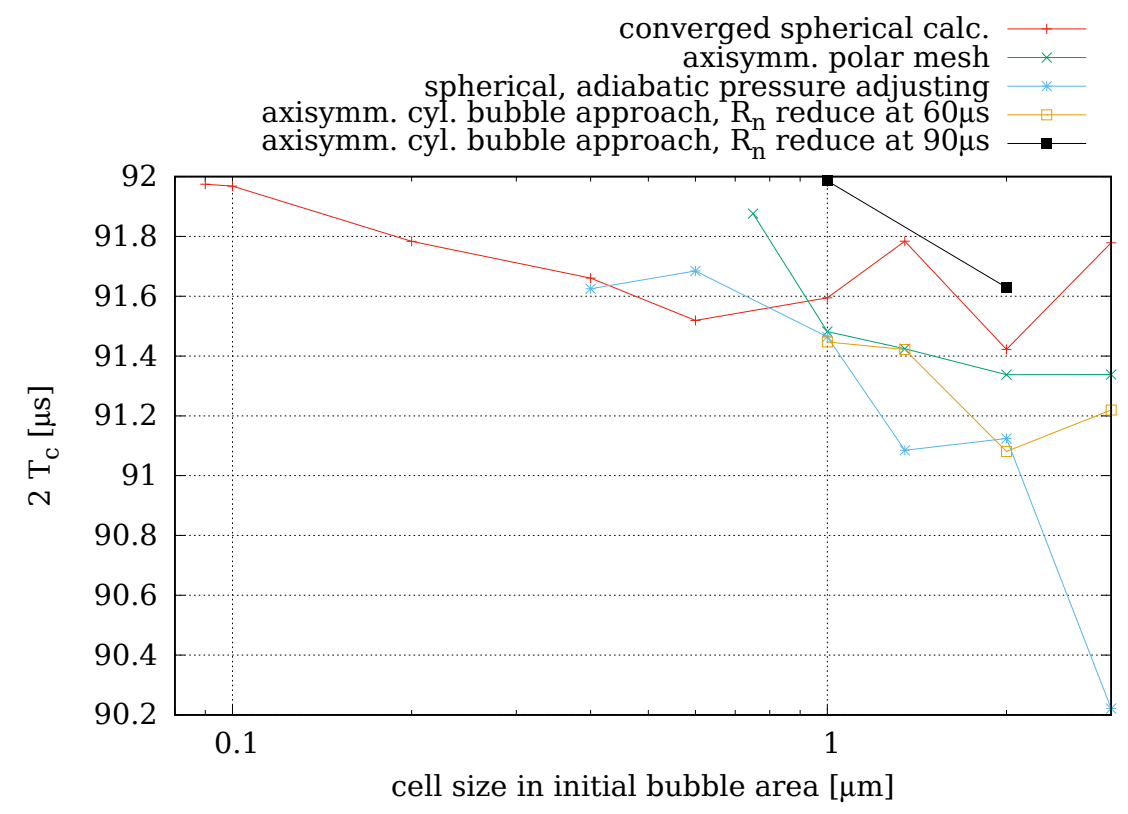

Fig. 4.10: Same diagram as Fig. 4.2, but including the distinguishing of the $R_{n}$-reduction interval times in the cylinder bubble approach.

It can be seen that more gas content inside the bubble prolongs the collapse time a bit and eventually pushes it more to the convergence value. Since the axisymmetric calculations are too time consuming for cell sizes lower than $1 \mu \mathrm{m}$, no statement about solution convergence can be done here, but it can be deduced that the time interval of $R_{n}$-reduction does not play a major role.

Figures 4.11 to 4.14 show the results for the $1 \mu \mathrm{m}$ calculation. Some more data points were added to the transition region from free vortex to wall vortex. Figure 4.15 then shows the vortex direction data points plotted with the data of Fig. 4.1. A qualitative comparison of the main differences can be summarized like the following:

\begin{tabular}{l|lll} 
& stationary vortex & vortex pureness & $\begin{array}{l}\text { free vortex appar- } \\
\text { ent speed }\end{array}$ \\
\hline \hline $2 \mu \mathrm{m}$, Sec. 4.1 .1 & at $D^{*}=0.4$ & mainly one major & stays in sight \\
\hline $1 \mu \mathrm{m}$, Sec. 4.1.2 & at $D^{*}=0.42$ & sometimes twofold & out of sight
\end{tabular}

Table 4.4: Summarizing qualitatively the differences between the $2 \mu \mathrm{m}$ and $1 \mu \mathrm{m}$ calculations. 
Without wall refinement:

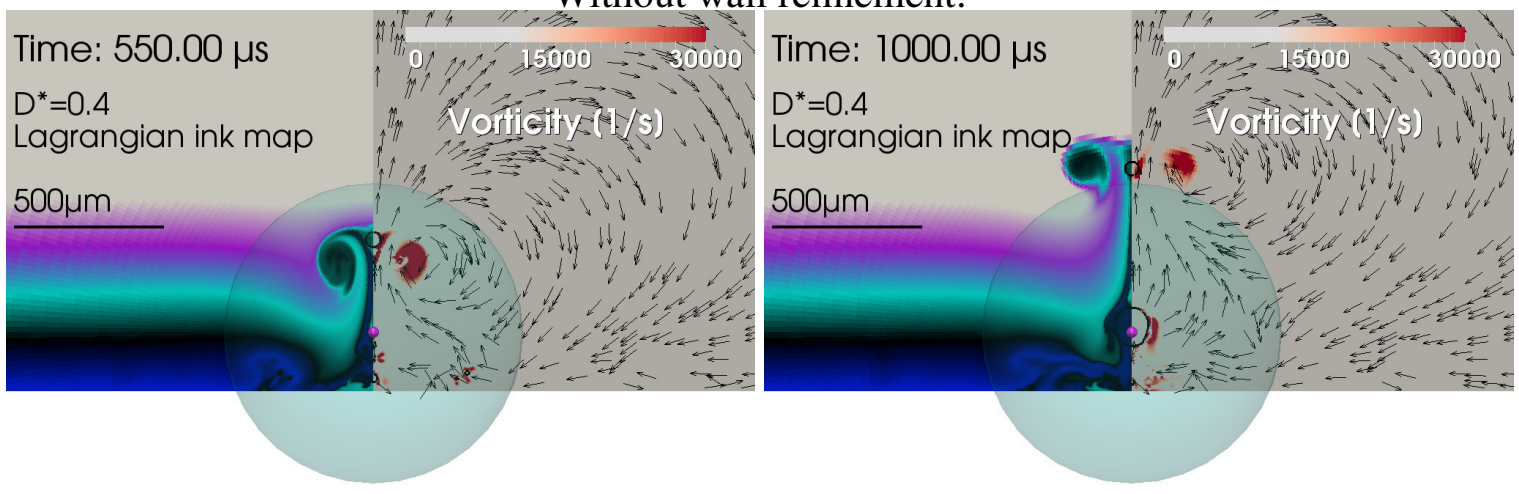

Without wall refinement:

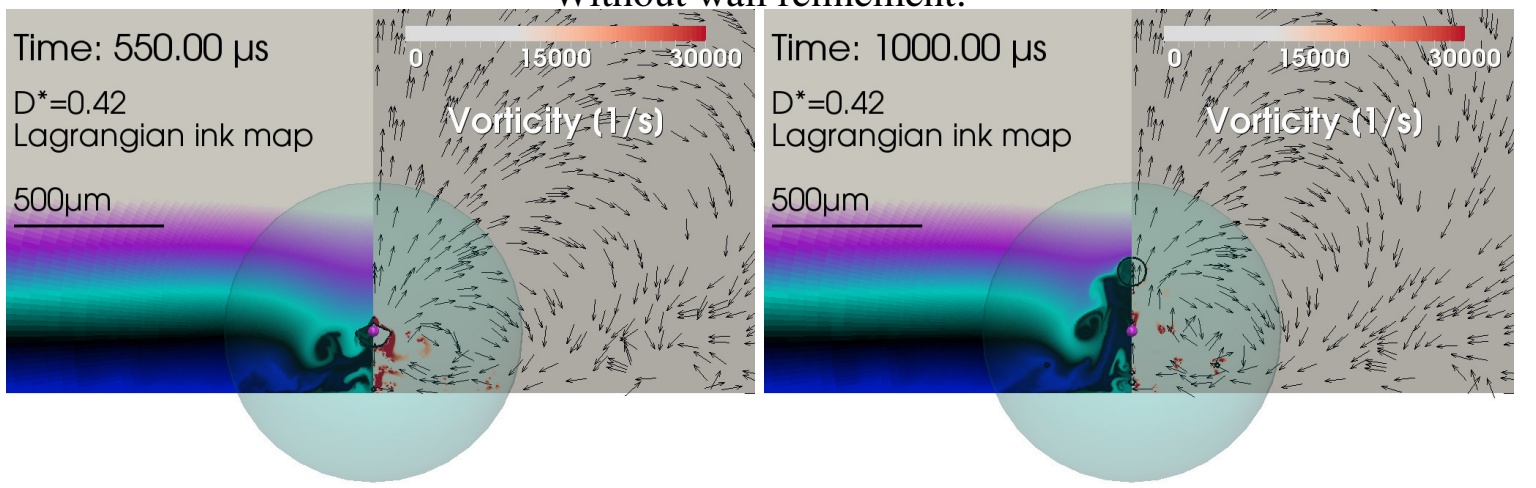

From here including wall refinement:
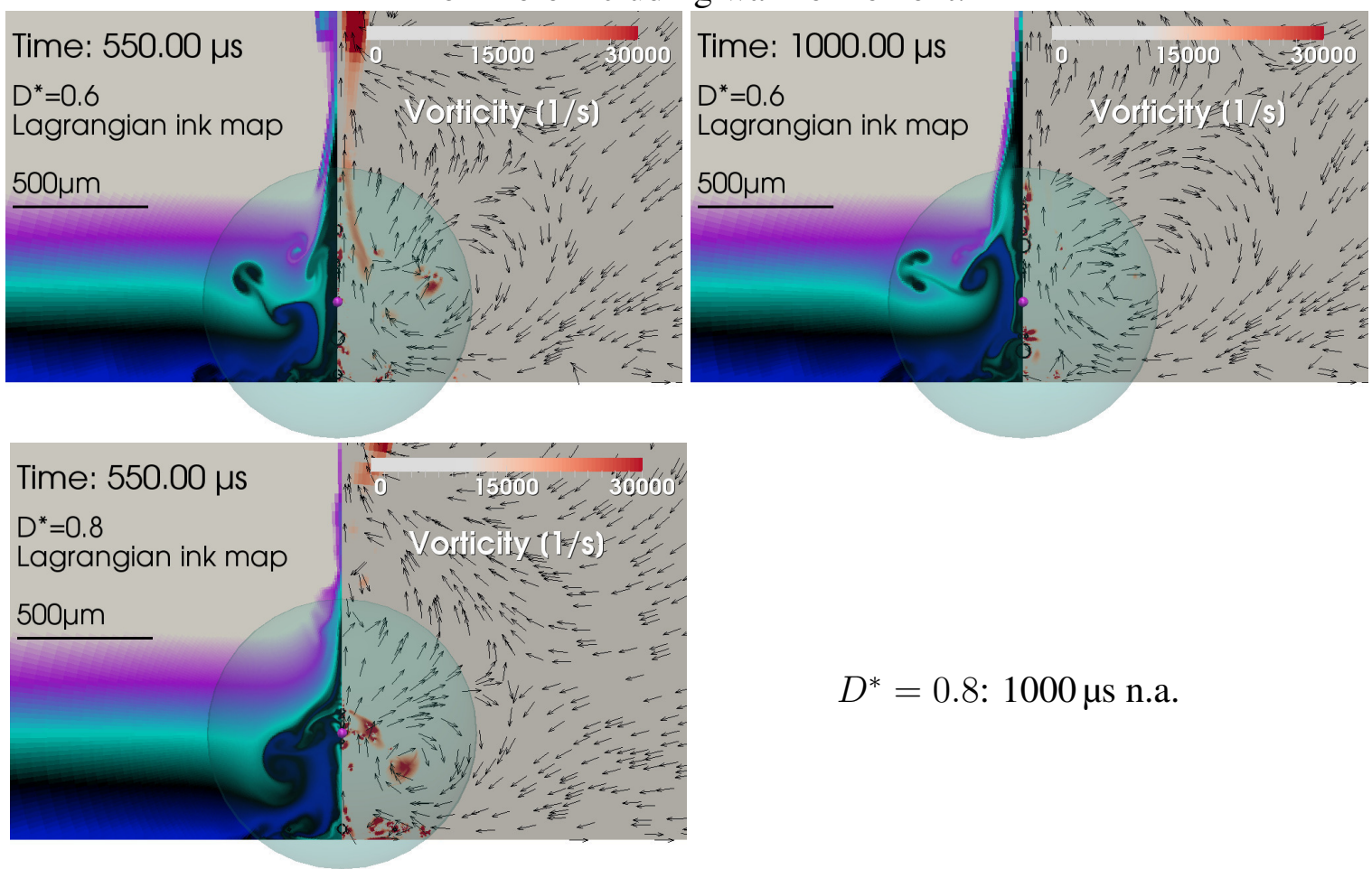

$D^{*}=0.8: 1000 \mu$ s n.a.

Fig. 4.11: Free and stationary vortices generated by the $D^{*} \in[0.4,0.42,0.6,0.8]$ bubbles, simulated with the $1 \mu \mathrm{m}$-approach for two very late time instants: $550 \mu \mathrm{s}$ (left) and $1 \mathrm{~ms}$ (right). In this approach, the Stationary vortex is more generated at $D^{*}=0.42$ than at $D^{*}=0.4$. The $D^{*}=0.8$ simulation crashed before reaching the limiting time. 


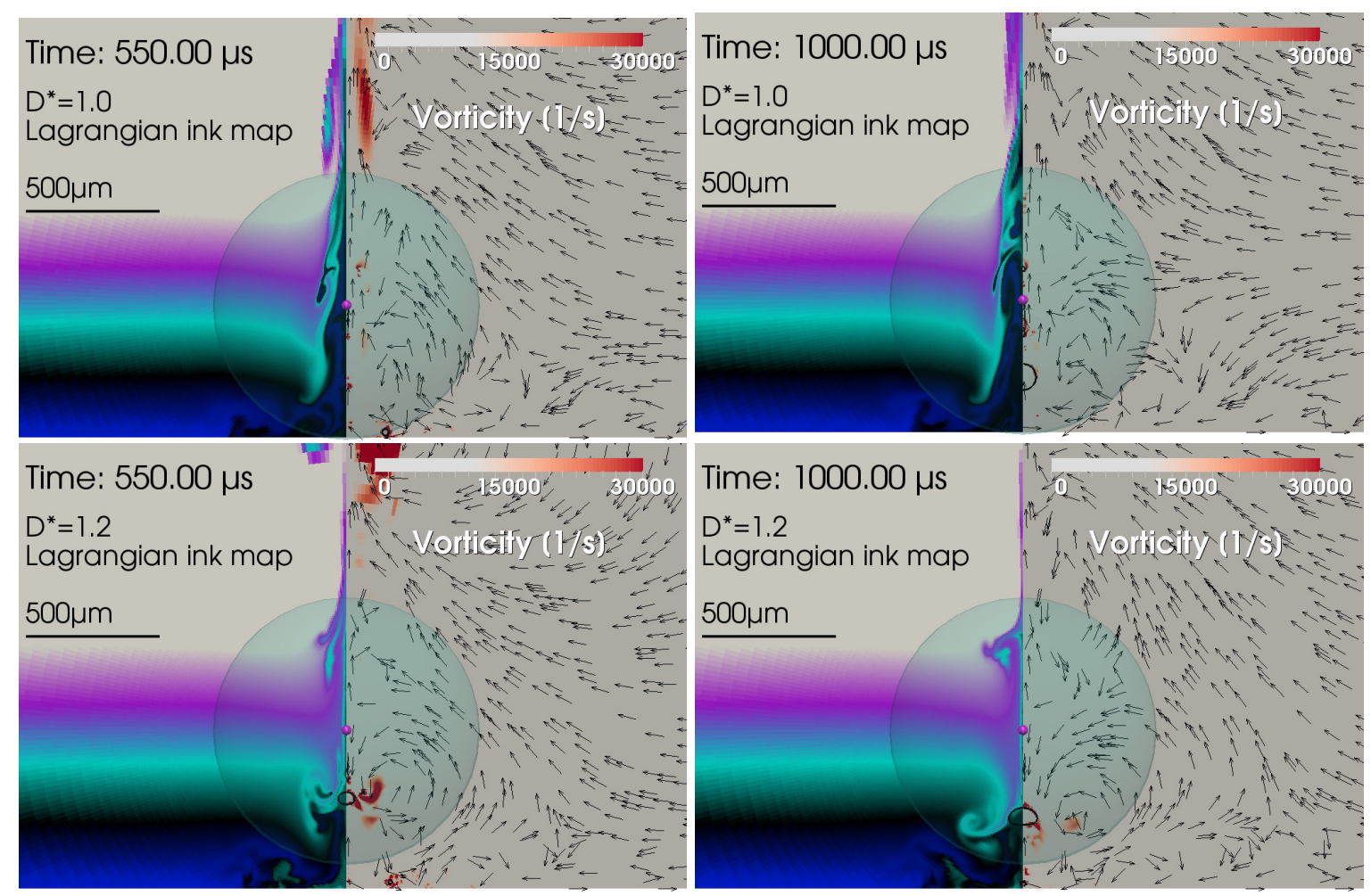

Transition from free vortex to wall vortex, without wall refinement:

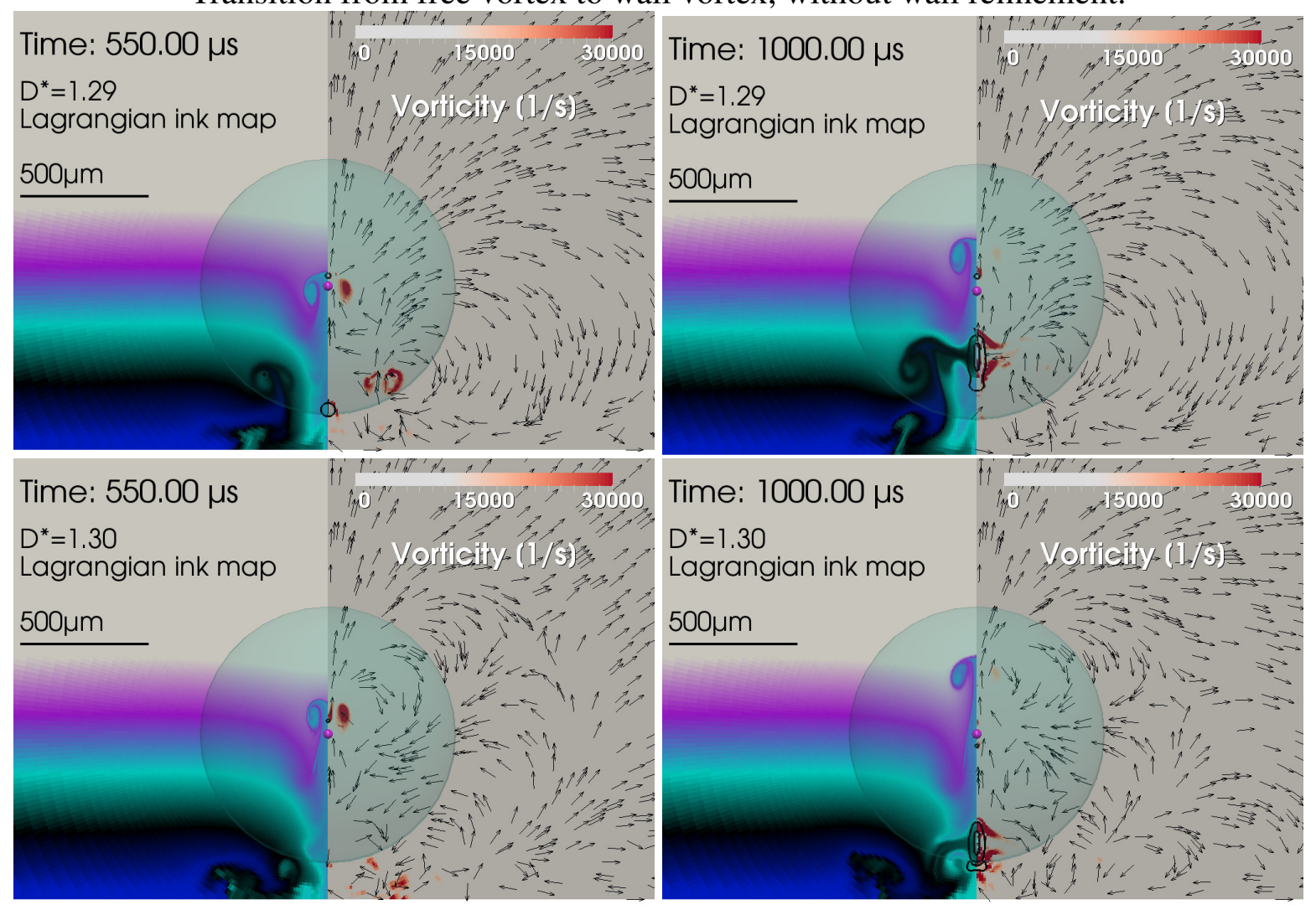

Fig. 4.12: Free vortices and the transition to wall vortex generated by the $D^{*} \in[1.0,1.2,1.29,1.30]$ bubbles. 


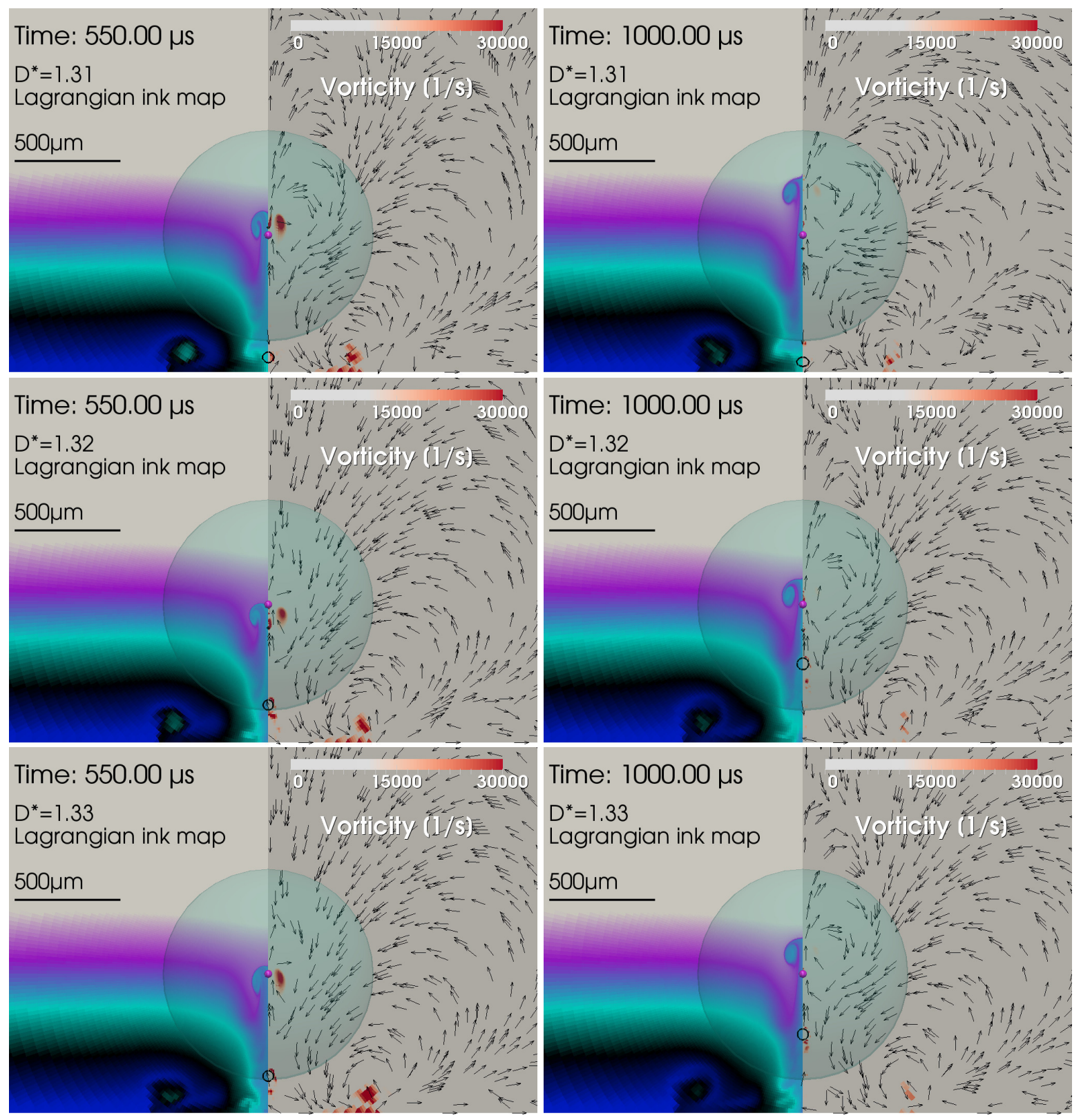

Fig. 4.13: Wall vortices generated by the $D^{*} \in[1.32,1.33]$ bubbles and a transition vortex at $D^{*}=1.31$. 


\section{Bubble dynamics at the planar, solid boundary}

From here again including wall refinement:

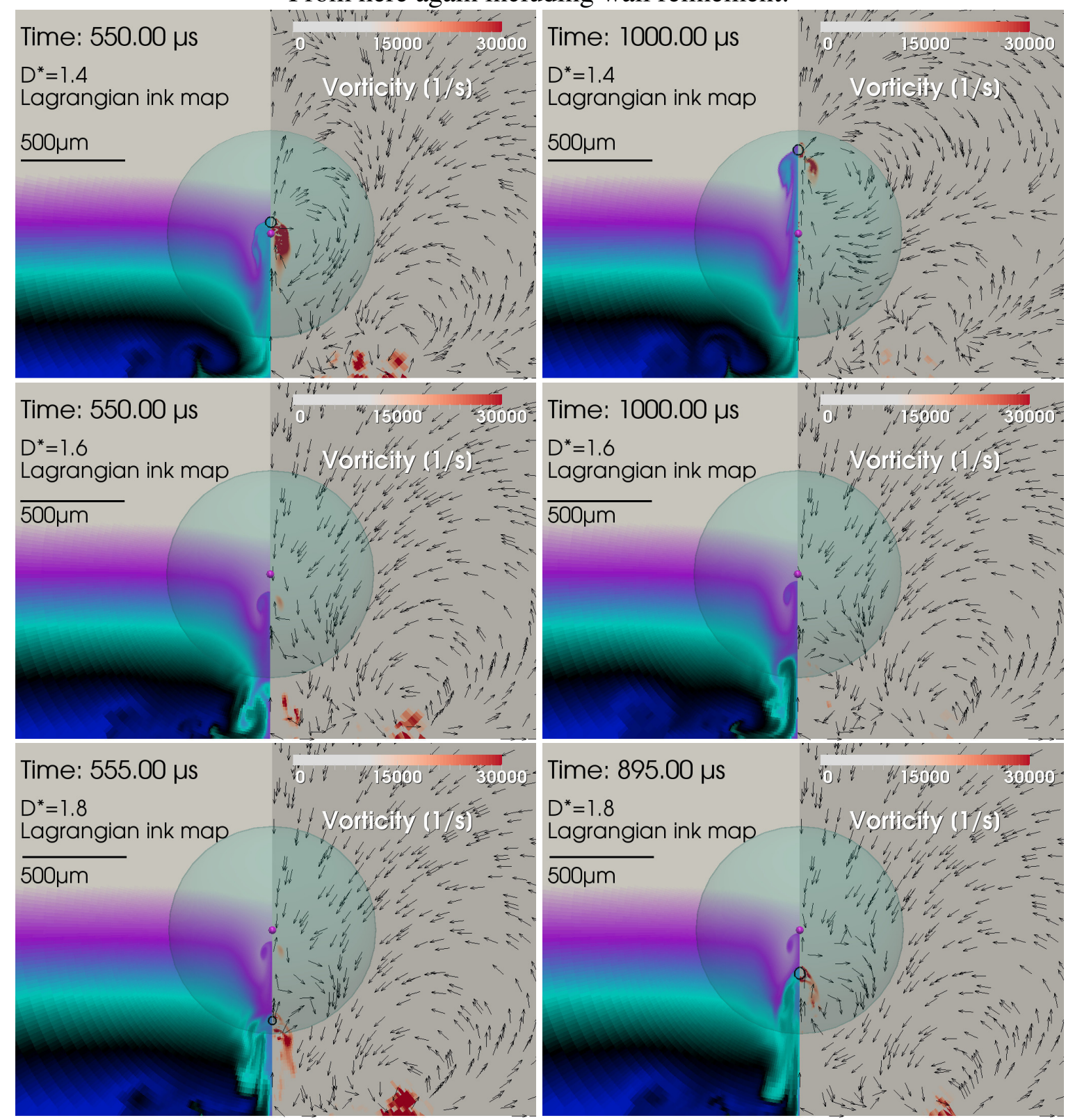

Fig. 4.14: Wall vortices generated by the $D^{*} \in[1.4,1.6,1.8]$ bubbles.

The evaluation of the vortex direction is not as clear with the $1 \mu \mathrm{m}$ calculation as with the $2 \mu \mathrm{m}$ calculation. Therefore, a floating point criterion is introduced:

$$
v_{d}= \begin{cases}1 & \text { clear free vortex } \\ 0.5 & \text { stationary vortex till } 1000 \mu \mathrm{s} \\ 0 & \text { clear wall vortex } \\ \text { else } & \text { gradual variation between the three }\end{cases}
$$

The cases where it is not fully clear are the bubbles at $D^{*} \in[0.4,1.29,1.30]$. Their values have been decided to be:

$$
v_{d}\left(D^{*}=0.4\right)=0.75, \quad v_{d}\left(D^{*}=1.29\right)=0.4, \quad v_{d}\left(D^{*}=1.3\right)=0.1
$$


At this point it is also discussable how to convert $D^{*}$ to $\gamma_{d}$. As stated in the introduction of Sec. 4.1, the authors determined the distance $d$ of the bubble from the solid boundary with the first available recorded image from the camera and the maximum radius is taken from the maximum bubble volume. From the numerical calculations the maximum volume is determined precisely and the distance from the solid boundary is taken either at $4 \mu$ s or at $10 \mu$ s to stress the influence of the time instant here. The values are fit with the function

$$
\gamma_{d}=a\left(D^{*}\right)^{b}+c .
$$

The results are plotted in Fig. 4.15. It is seen that the two different conversion rules mainly influence the data points where $D^{*} \leq 1$. However, the main result that the vortex direction changes with $D^{*} \approx 1.29$ remains untouched. Furthermore the following conclusions can be consolidated:

1. The vortex phenomenon is well captured by the solver, thus long-term dynamics can be resolved as well.

2. The vortex phenomenon does not necessitate a full 3D calculation, hence the main, global aspect of the dynamics of the phenomenon is axisymmetric.
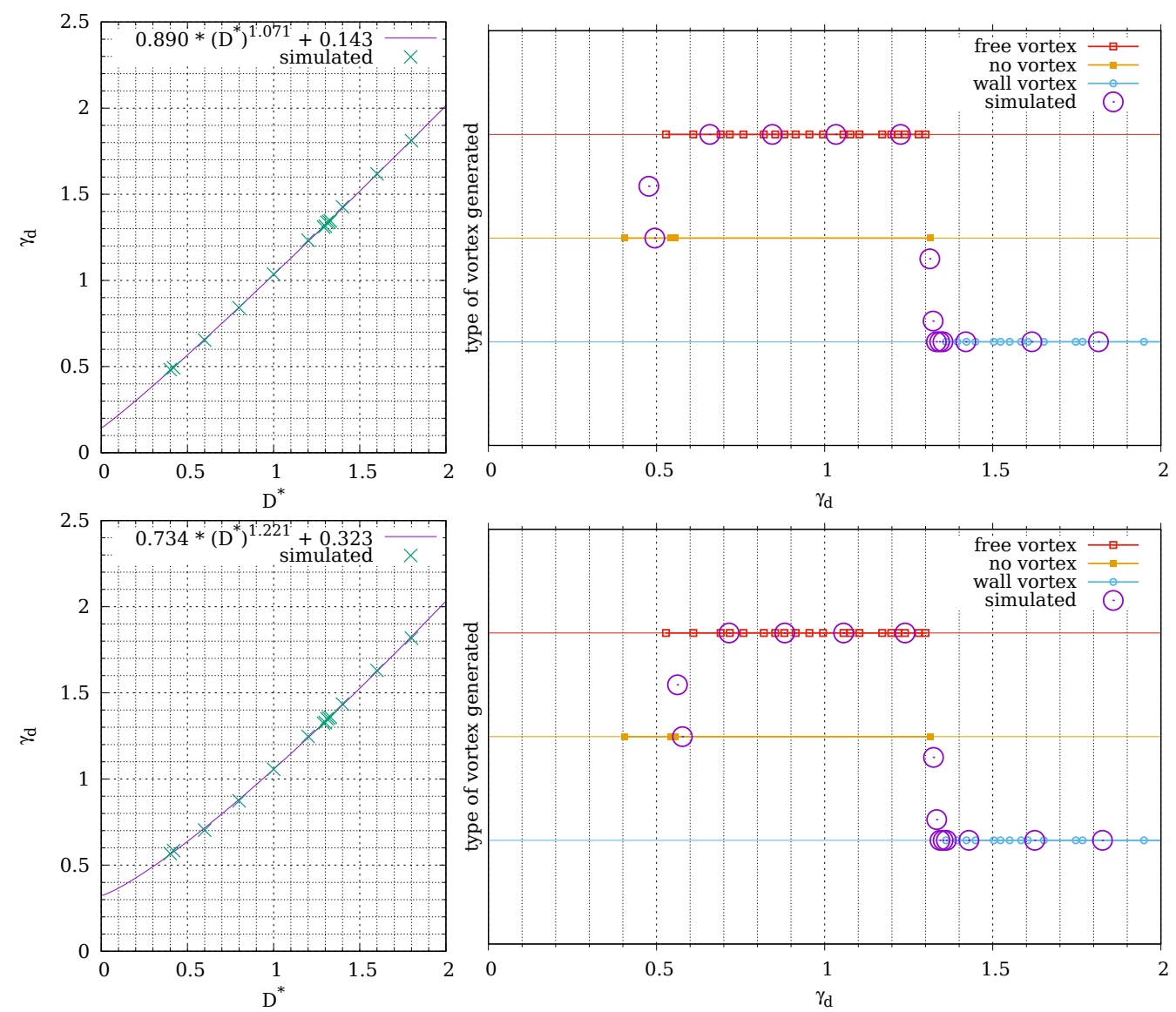

Fig. 4.15: Left: Non-linear fit for converting $D^{*} \rightarrow \gamma_{d}$ with rules

$\gamma_{d}=d(t=4 \mu \mathrm{s}) / R_{\max }\left(t=t\left(V_{\max }\right)\right)$ (top) and

$\gamma_{d}=d(t=10 \mu \mathrm{s}) / R_{\max }\left(t=t\left(V_{\max }\right)\right)$ (bottom). Data for vortex experiments from

(Reuter et al. 2017a). Depending on the $D^{*}\left(\gamma_{d}\right)$-rule, the data points for low normalized distances shift in $\gamma_{d}$-direction. 


\subsection{The jet-illusion bubble}

(This section is similar to parts in Koch et al. (2020). An updated, more detailed and more quantitative investigation is found there.)

The jet-illusion bubble serves as an example to show that refraction can be deceiving and apparently triplicate the jet velocity. The overlay-method can be used to infer the precise shape of a strongly aspherical bubble and correct the jet velocity value, even though the underlying simulation disregards 3D effects that might result from the small confinement of the cuvette.

As an example of the expected dynamics, distinct time steps of a simulation performed with the code described in Sec. 2.4.1 are shown in Fig. 4.16. The laser-induced breakdown creates an elongated plasma which is modelled by an initial gas cylinder of high internal pressure at a distance of $216 \mu \mathrm{m}$ to the wall, resulting into a dimensionless distance of $D^{*}=0.332$ (Eq. 3.1). Afterwards the bubble expands and collapses including an involution of its surface because of the hindered water inflow from the boundary side. The cross-section of the bubble is shown in Fig. 4.16, together with the refraction of a pattern of 17 straight illumination stripes of alternating color (white, yellow and pink) behind the bubble. Each stripe has dimensions of $50.8 \mathrm{~mm} \times 1 \mathrm{~mm}$ in length and width. The stripe-to-stripe distance is $1.6 \mathrm{~mm}$ and the pattern is put in a distance of $10 \mathrm{~mm}$ behind the bubble, ergo $5 \mathrm{~mm}$ behind the cuvette. The overall size of the pattern is $50.8 \mathrm{~mm} \times 26.8 \mathrm{~mm}$. In one of the plots the pattern is put in vertical, in the other frame in horizontal orientation. The vertical and horizontal pattern alignment is ray-traced and compared to the cross-section of the bubble. Illumination grids or patterns are used for example to correct lens aberration (Nobach 2012). The pattern deformation gives insight into the refraction distortion. In contrast to lenses, the distortion here is beyond linear approximations. It can be seen that for the time of interest, i.e., the jetting phase around $139 \mu$ s to $142.6 \mu \mathrm{s}$, it is challenging to get an illumination configuration where the jet and bubble interior appear clearly separated. That is why the illumination device has to be designed and placed accurately. The circular geometry of the flash tube in the experiment fulfilled this requirement by chance.

With the Imacon 468 camera the involution (liquid jet) was recorded. One can obtain 8 images only, but at very high frame rate (down to $10 \mathrm{~ns}$ inter-frame time). Hence, the higher the frame rate, the better the triggering circuit must be. Here, some of the best results are shown that could be obtained by manually varying the delay time until the camera trigger happens to exactly match the time of jet formation during the bubble collapse phase.

The results in Fig. 4.17 were obtained with $150 \mathrm{~ns}$ exposure time and an inter-frame time of $500 \mathrm{~ns}$ between the end of the previous frame and start of the next frame. The glass surface wall is located at the bottom of the frames, indicated by the red line in the first frame, so the image is rotated by $90^{\circ}$ compared to the setup sketch in Fig. 3.16. The first frame shows the plasma formation at the beginning. The times and spatial scale are indicated within the frames. The jet formation is seen clearly by a dark shadow piercing the bubble from top to bottom and widening over time. There seem to be two windows of the outer bubble interface where the jet is seen through. One big window in the middle and a narrower at the bottom. In the latter, the jet only is visible from frame $6(t=112.1 \mu \mathrm{s})$ onward, although it has pierced the bigger window already in frame 3. To conclude, there is some nonlinear distortion of the apparent jet speed by refraction. If the experiment was evaluated only by pixel counting, the following minimum jet speed would be found, taking the time between the end of the third frame and the end of the 


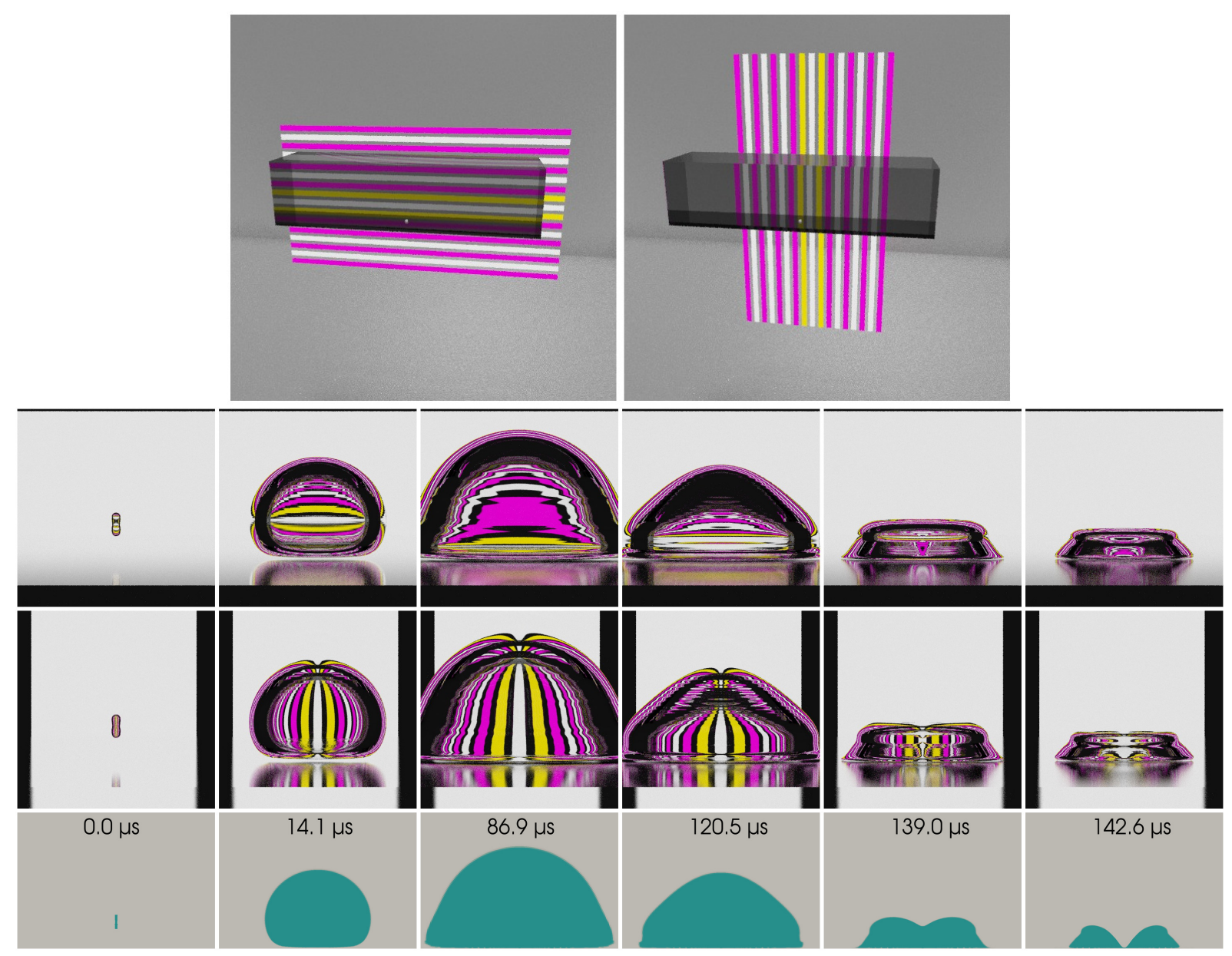

Fig. 4.16: Ray-tracing of a regular, symmetric illumination pattern with alternating color (pink and white - yellow next to the center) behind the small cuvette shown here with darker glass and lighter bubble for better contrast (first row). Note that the cuvette is rotated by $90^{\circ}$ to the left compared to Fig. 3.16. Simulated bubble with illuminating rectangle in horizontal alignment (second row) and in vertical alignment (third row). The bubble is mirrored in the glass surface. Fourth row shows the bubble cross-section (not ray-traced). Frame width of the ray-traced bubbles is $1162 \mu \mathrm{m}$, the one of the cross-section is $1600 \mu \mathrm{m}$. 


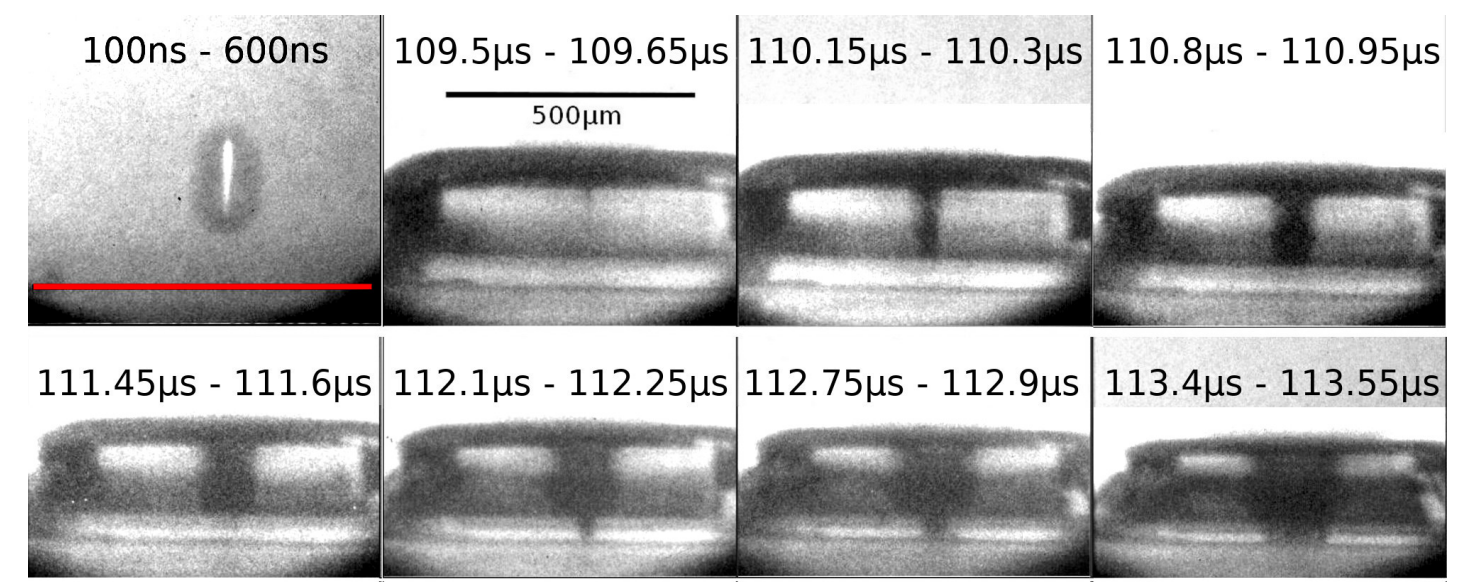

Fig. 4.17: Liquid jet piercing the bubble, captured at 1.538 Mfps. The first frame shows she plasma of the laser-induced breakdown. The red line in the first frame indicates where the solid boundary (glass) is located in the frames.

second frame of the top row:

$$
\frac{500 \mu \mathrm{m} / 352 \mathrm{px}}{110.3 \mu \mathrm{s}-109.65 \mu \mathrm{s}} \cdot 80 \mathrm{px} \approx 175 \mathrm{~m} / \mathrm{s}
$$

The appearance of light and shadow features highly depends on slightest changes of the interface curvature due to the nonlinearity in Snell's law.

For determining the correct bubble shape from the photographs in Fig. 4.17, the bubble contour from the numerical simulation in Fig. 4.16 is taken as a basis. The simulation gives a bubble involution dynamics as shown in Fig. 4.18. The contour now is imported into blender

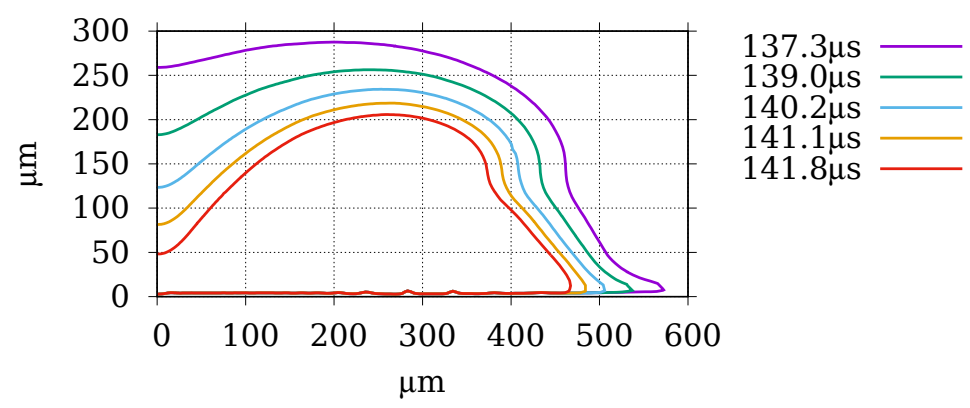

Fig. 4.18: The contour lines of half of the cross-section of the numerical bubble in Fig. 4.16 for several instants of time during jetting. Axis of symmetry is located on the left side.

and reduced in grid complexity while keeping the shape. It is then rotated and extruded around the axis of symmetry. The curvature of the contour profile curve determines the curvature of the resulting object, the bubble probe. The shape of the bubble probe can be adopted such that it resembles the experimental one to high level of detail by manually altering parts of the profile curve while watching the ray-tracing image outcome. This process has been applied to the bubble contour from Fig. 4.18 and is shown in Fig. 4.19.

Taking all light and shadow features into account, the bubble shape to obtain highest correlation becomes quite definite. In some parts of the profile curve even a change in inclination by only a fraction of a degree results, e.g., into a fully light or half dark bubble interior. An 


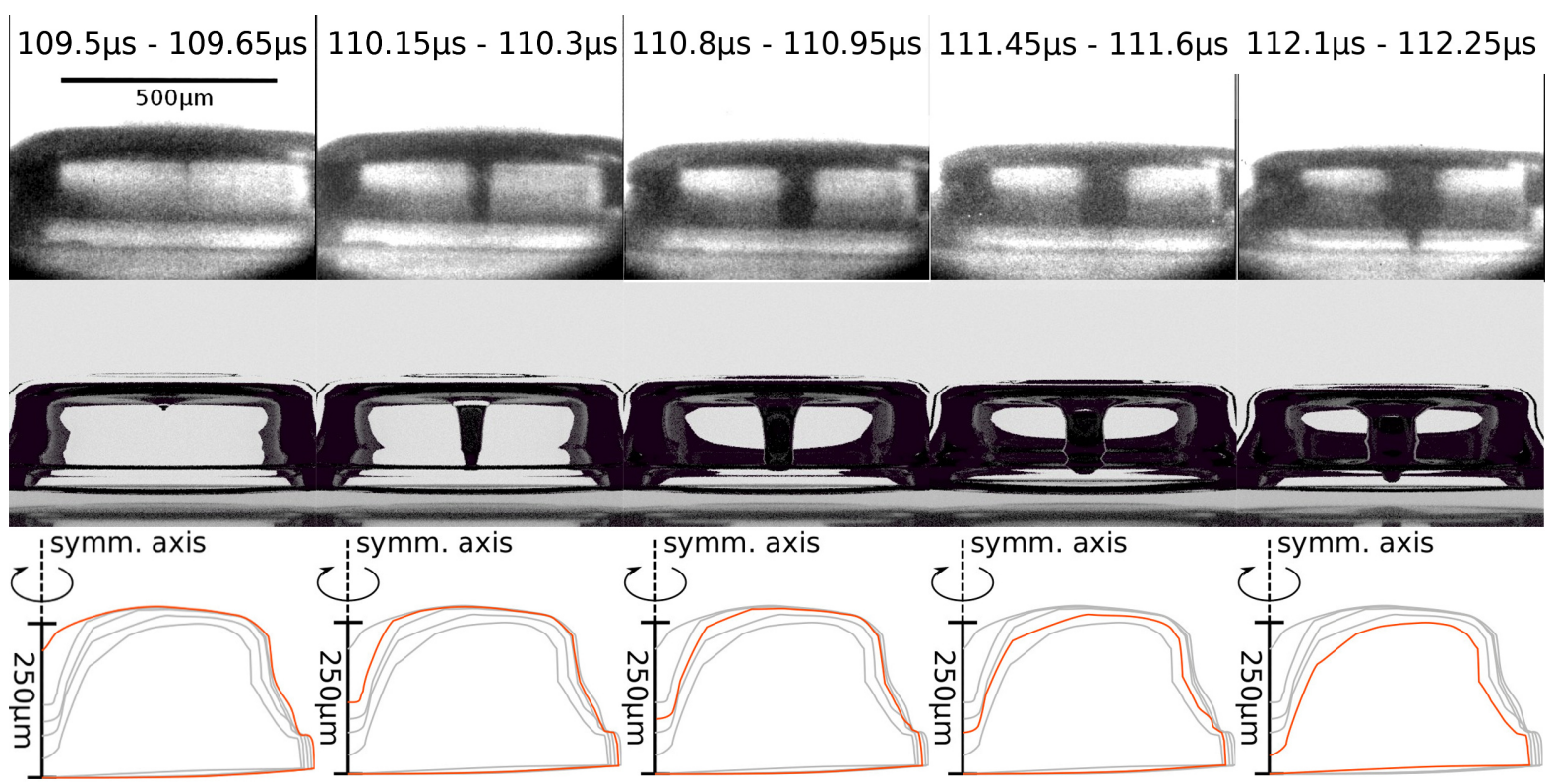

Fig. 4.19: The jet-speed illusion. Top row: experimental photographs. Second row: ray-tracing of the manually adjusted profile that is defined by the curve shown in the third row.
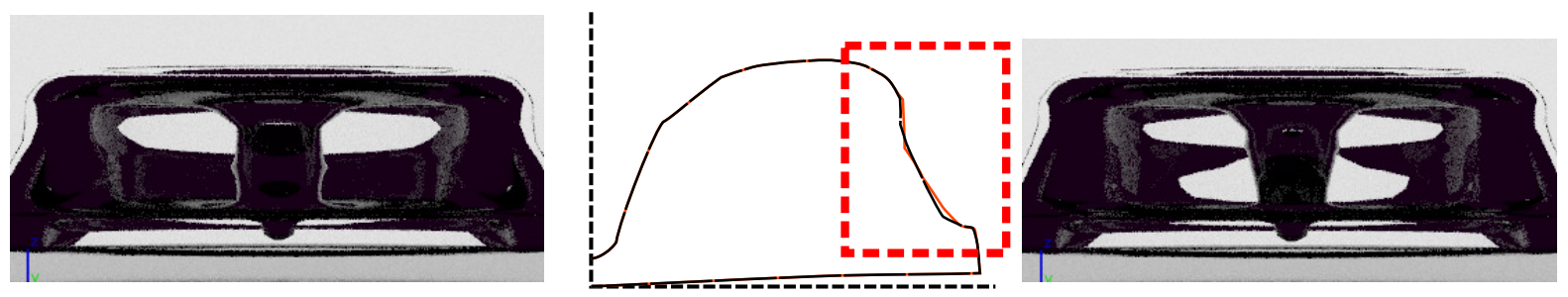

Fig. 4.20: Example of the effect of a slight change of the bubble interface shape. The black contour line produces the second ray-tracing frame, whereas the original orange line produces the first frame.

example of the sensitivity of the shape is given in Fig. 4.20, where the effect of a slight change in the interface curvature changes the image.

Now the jet velocity can be recalculated using the bubble contours in the bottom row of Fig. 4.19:

$$
\frac{250 \mu \mathrm{m} / 246 \mathrm{px}}{110.3 \mu \mathrm{s}-109.65 \mu \mathrm{s}} \cdot 87 \mathrm{px} \approx 57 \mathrm{~m} / \mathrm{s},
$$

giving approximately one third of the value that was derived from the photographs.

It is the first time known to the author that the shape of a jetting cavitation bubble was inferred from the experiment with such high precision. With the successful application of the overlay-method, many more possibilities arise. One could think of, e.g., a specialized setup with an illumination grid in order to deduce the bubble shape automatically via an algorithm. Furthermore, it opens up the possibility to validate CFD codes to an unprecedented level of detail. 


\section{The quest for the fast jet}

As mentioned before, a peculiar jetting dynamics was found numerically in the studies by Lechner et al. (2019) and Lechner et al. (2020) for $D^{*} \leq 0.2$. While the bubbles for $D^{*} \geq 0.24$ exhibit a "normal" microjet by involution of the top bubble wall, these ones exhibit jets that are one order of magnitude faster and are produced by an annular inflow of liquid towards the axis of symmetry. The latter work with the complete numerical investigation of this phenomenon (Lechner et al. 2020) has been published in September 2020 after a long review process since the 20th June 2019 because the community was hard to be convinced that the findings should be reported. Since 100 years it is known that bubbles erode even hardest materials but till now there is little evidence of forces in that magnitude. Over the years it got silently accepted. The fast jet accelerates to about $1000 \mathrm{~m} / \mathrm{s}$ within $20 \mathrm{~ns}$. For the first time these are numbers that seem plausible for erosion and yet the fact is rejected by fellow scientists. Before going on, a philosophical question has to be posed concerning the basic scientific practice of numerical simulation:
After performing successful convergence studies and interpolation of existing ex- perimental results, numerical simulations are there to extrapolate experiments and gain knowledge in parameter sub-spaces where experimental results don't exist. How scientifically correct or incorrect then is it to reject the publishing of the re- sults of the extrapolation (incl. rejection of the possibility of a rebuttal process) or to claim for an experimental validation again?

In this thesis simulations in full 3D and experimental investigations, partly used in Koch et al. (2020), are added to the work of Lechner et al. (2020). The results are:

1. the fast jet phenomenon also occurs in a full 3D simulation, thus falsifying the possibility of it being a numerical artefact of axial symmetry

2. the experimental data alone is not convincing but

3. using the overlay method gives strong indication of its existence

\subsection{Bubble dynamics at $D^{*}=0$}

In Figs. 5.1 and 5.3, a simulation in axial symmetry is performed for a $D^{*}=0$ bubble with parameters

$$
R_{\text {init }}=20 \mu \mathrm{m}, D_{\text {init }}=0 \mu \mathrm{m}, R_{\max }=575 \mu \mathrm{m}, R_{n, 1}=220 \mu \mathrm{m}, R_{n, 2}=64 \mu \mathrm{m} .
$$

At $D_{\text {init }}=0$ half of the initial bubble volume is lost, which is automatically accounted for by using algorithm 2 of Tab. 3.1 for the initial data. The bubble parameters are chosen such that the collapse time fits the experimental bubble of Fig. 5.2. The Mesh 3.1.2.a is used, but without 
the lower half in the beginning already, skipping the snappyHexMesh part. The geometric parameters are:

$$
\delta x_{\min }=1.5 \mu \mathrm{m}, \mathrm{Xi}=121.5 \mu \mathrm{m}, \mathrm{Xii}=2 \mathrm{Xi}, \mathrm{X}=1.2 R_{\max }, g_{f}=5, \mathrm{XF}=100 R_{\max } .
$$

The distance Xi had to be set further outwards compared to standard $80 \mu \mathrm{m}$, in order to increase the angular resolution of the mesh. This is necessary to have enough resolution to resolve the curvature of the bubble at $R=R_{\max }$. In Fig. 5.1 the pressure (left) and liquid velocity (right) are plotted for the times of the experimental recording in Fig. 5.2.

Note: The evaluation of the absolute values of the jet velocities is done later in Ch. 6.

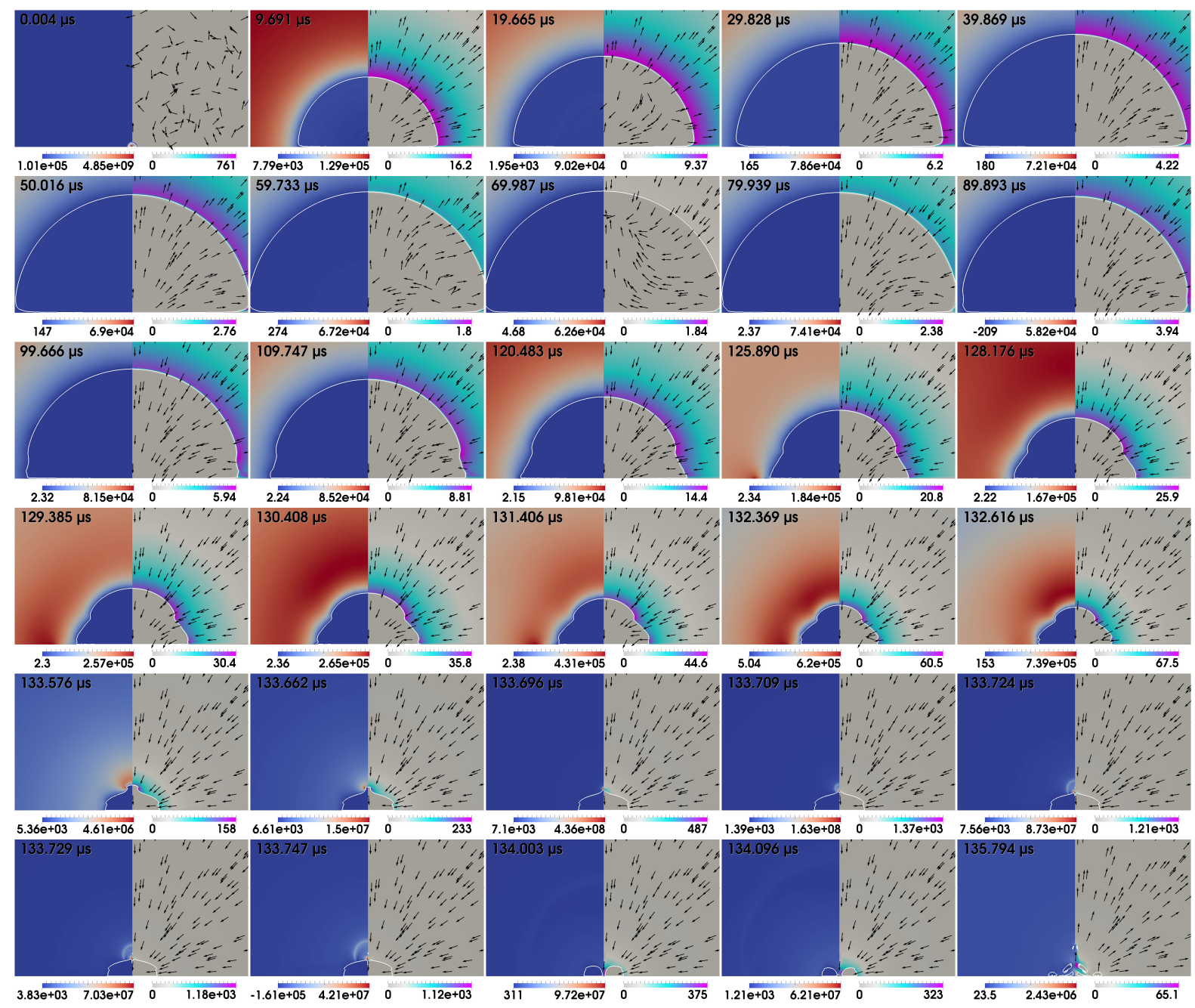

Fig. 5.1: Simulation of a $D^{*}=0$ bubble in axial symmetry. Bubble expansion and collapse at time instants fitting the images from the experiment in Fig. 5.2. Left side of the frames is pressure in Pascal $(\mathrm{Pa})$, right side liquid velocity in $\mathrm{m} / \mathrm{s}$. 
5 The quest for the fast jet

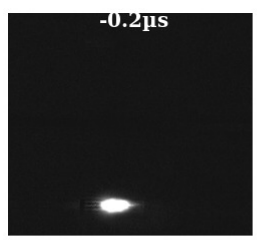

0.004ps

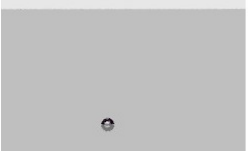

49.8us

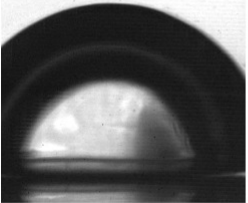

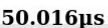

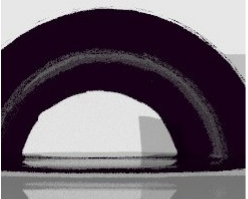

99.8us

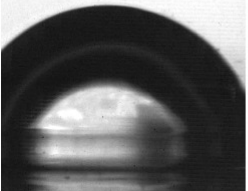

99.666ps
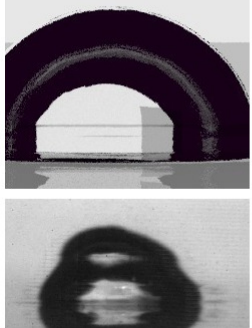

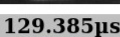
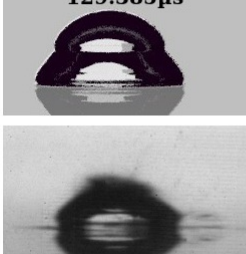

133.576us
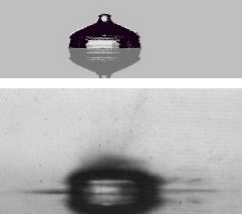

133.729ps

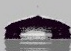

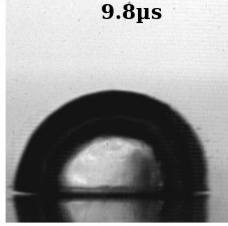

$9.691 \mu \mathrm{s}$

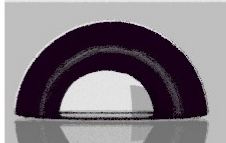

$59.8 \mu \mathrm{s}$

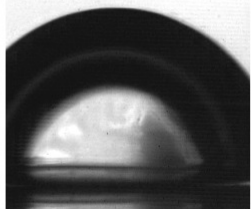

59.733us

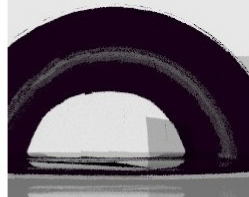

109.8ps

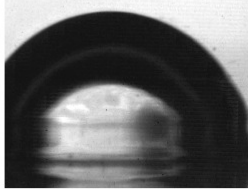

109.747ps
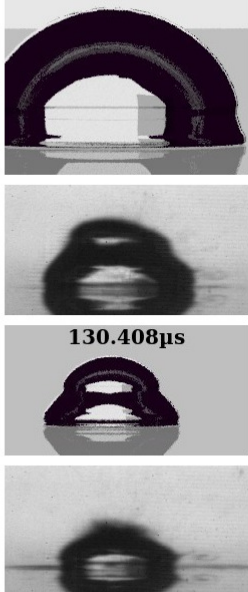

133.662us
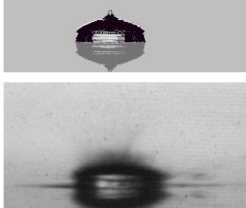

133.747ps

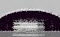

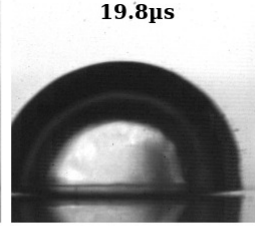

$19.665 \mu$

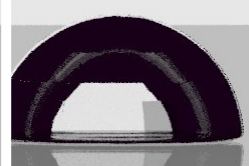

$69.8 \mu \mathrm{s}$

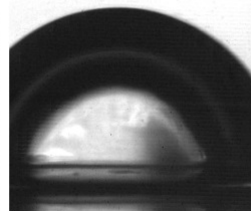

$69.987 \mu s$

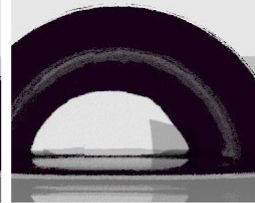

119.8us

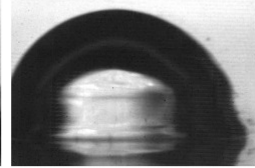

120.483ps
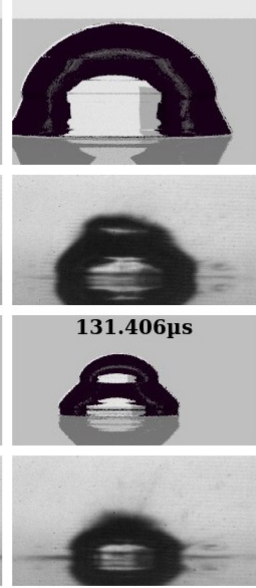

133.6961s
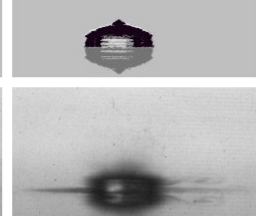

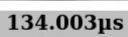

$\infty$

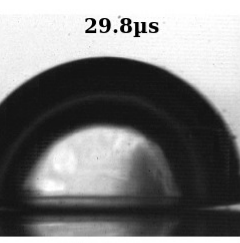

29.828ps

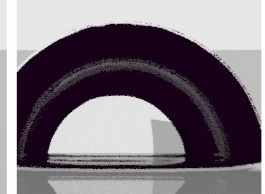

79.8us

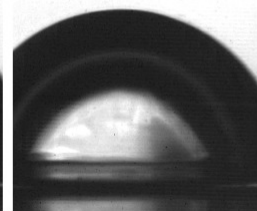

79.939ps
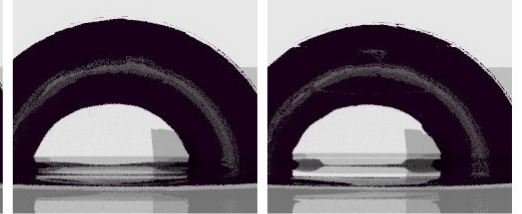

129.8ps

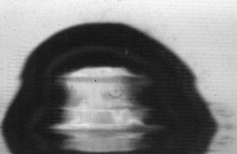

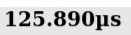
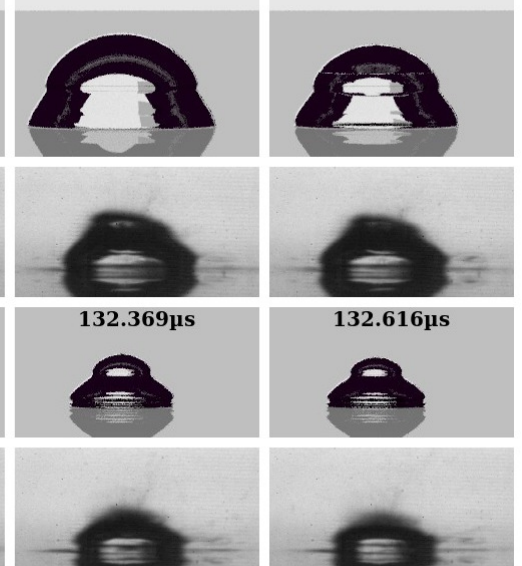

133.709ps
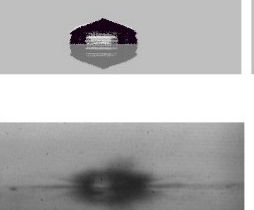

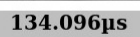

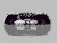

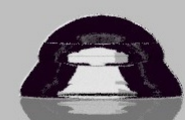

128.176ps
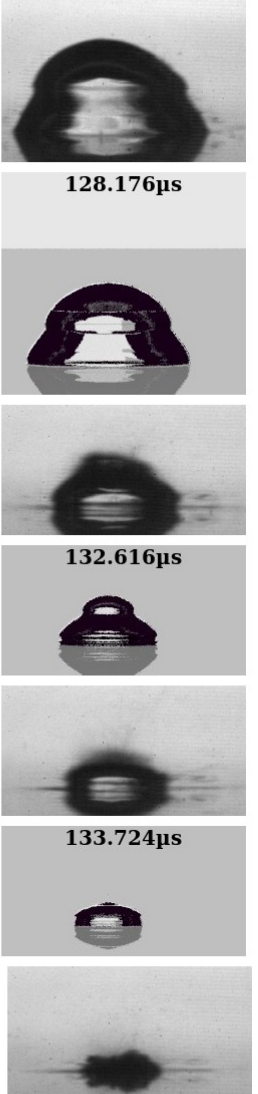

135.794ps

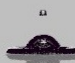

Fig. 5.2: Overlay of the simulation of Fig. 5.1 (even rows) and experimental recordings (odd rows) using setup 1 (Sec. 3.3.1) for a $D^{*}=0$ bubble. The experimental images with a time annotation belong to the same measurement, the remaining 16 experimental frames are from 15 different measurements. Frame width is $1048 \mu \mathrm{m}$. 

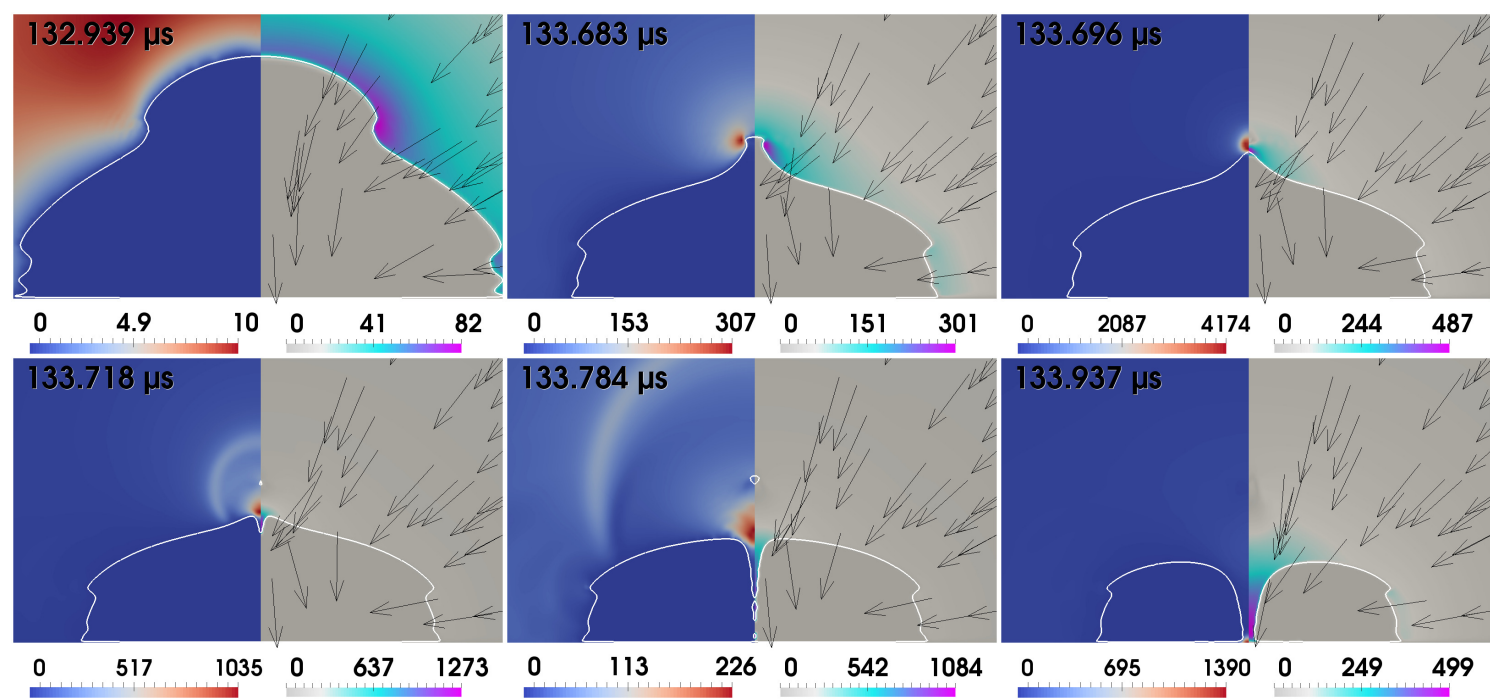

Fig. 5.3: Simulation of a $D^{*}=0$ bubble in axial symmetry. Zoom into the time of jetting of the bubble in Fig. 5.1. Left is pressure in bar, right liquid velocity in $\mathrm{m} / \mathrm{s}$

a)
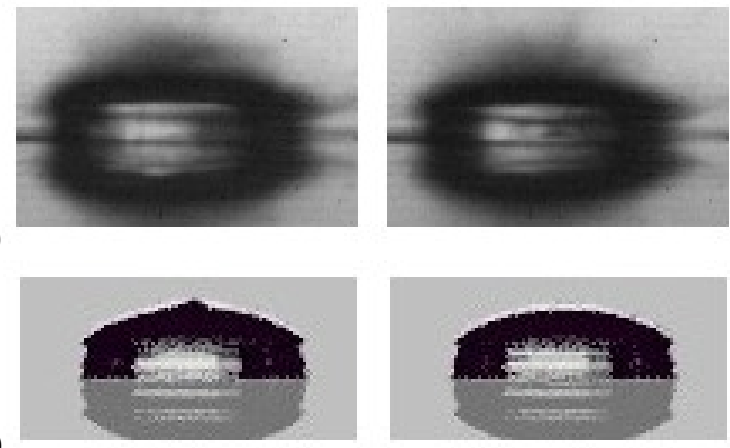

b)
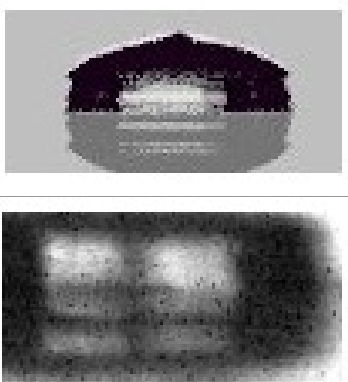

c)

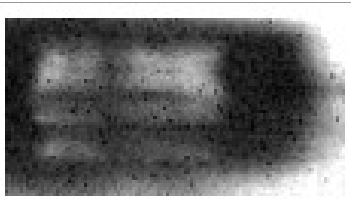

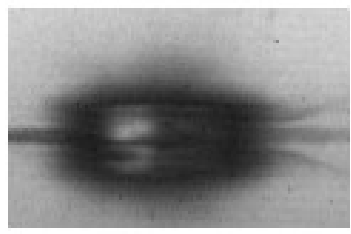
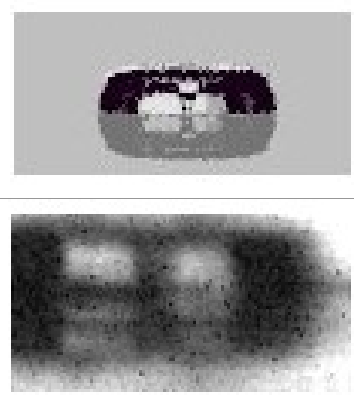
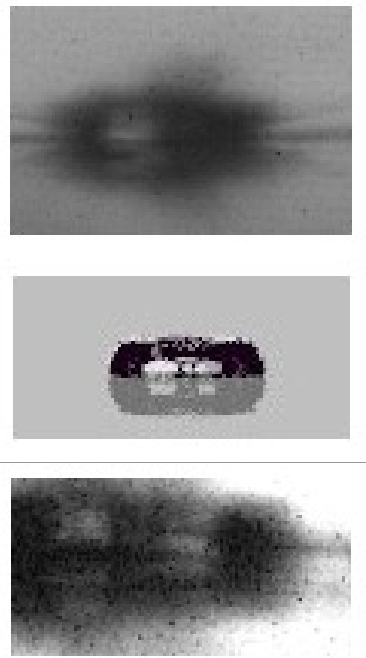

Fig. 5.4: Zoom into experimental frames (a) from Fig. 5.2, last row and the respective ray-traced simulation (b). (c): From 51 measurements using setup 1 (Sec. 3.3.1) with $400 \mathrm{kfps}, 4$ measurements showed a jet shadow.

In Fig. 5.2, the experimental recordings using setup 1 (Sec. 3.3.1) with $100 \mathrm{kfps}$ recording speed are compared to the ray-traced images of the simulation of Fig. 5.1. The measurements were quite repeatable, so the images of different measurements could be stacked. The illumination timing was synchronised to the $1 \mu$ s shutter of the camera such that less than the full LED flash time $(1 \mu \mathrm{s})$ matches the shutter time and therefore reducing the illumination time below $1 \mu$ s to approximately $500 \mu$ s. The frame width has not been noted down or measured, but because the simulation fits very well in size and time, it could be calculated retrospectively. The height of the numerical bubble at $t=99.666 \mu \mathrm{s}$ is $670 \mu \mathrm{m}$ and therefore the frame width is $1048 \mu \mathrm{m}$. The plasma was generated directly on the metal surface, the laser entering from the top of the frames. These measurements had been discarded for long since the experimental 


\section{The quest for the fast jet}

bubble shows an asymmetry in the dynamics and $100 \mathrm{kfps}$ were found to be too less. However, knowing the results further down in this chapter, the match in the overlay is much more apparent than was at the time of experiment conduction. The jet shadow back then was considered to be non-distinguishable from illumination inhomogeneities and jokingly called a ghost. However, when looking at the magnifications in Fig. 5.4, a) and b), the overlay shows that even for a perfectly sharp image, the pixel resolution and perspective of viewing does not produce a high contrast jet. In Fig. 5.4c four out of 51 different measurements of the same setup but with $400 \mathrm{kfps}$ recording speed are shown that in one frame show a jet shadow. The frames show the full resolution and frame size of the camera at that recording speed.

\subsection{Full 3D simulation}

The full 3D simulation was performed for a bubble with standard parameters

$$
\begin{array}{r}
R_{\text {init }}=20 \mu \mathrm{m}, R_{n, 1}=184.1 \mu \mathrm{m}, R_{n, 2}=64 \mu \mathrm{m}, R_{\text {max,unbound }}=495 \mu \mathrm{m}, \\
R_{n} \text { reduction in interval }[30 \mu \mathrm{s}, 40 \mu \mathrm{s}], D_{\text {init }}=20.295 \mu \mathrm{m}
\end{array}
$$

and with initial data algorithm 0 from Tab. 3.1 on the polar 3D Mesh 3.1.2.d with standard parameters

$$
\mathrm{C}=40 \mu \mathrm{m}, \mathrm{H}=180 \mu \mathrm{m}, \delta x_{\min }=1.8 \mu \mathrm{m}, \mathrm{X}=1.2 R_{\max }, \mathrm{XF}=80 R_{\max }
$$

resulting into the total amount of $6,407,940$ cells. The time stepping was set such that the maximum flow Courant number is 0.1 , the maximum Courant number for the interface is 0.1 , the maximum acoustic Courant number is 8.0 , and 1 for $R / R_{n}=0.3$, and the maximum time step size is $50 \mathrm{~ns}$. With these parameters the simulation took 597,938 seconds $\approx 6.9$ days for $114.008 \mu$ s of virtual time on 30 threads on the Xeon-E5 machine (list of calculation machines and benchmarking see Sec. B.4). The continuation until $122.647 \mu$ s took another 500369 seconds $\approx 5.8$ days. The calculation was done in order to verify the existence of the fast jet in 3D as well. Figure 5.5 shows the moment of fast jet $(t=113.52 \mu \mathrm{s})$ in a scientific visualization. The left frame shows the 3D bubble contour together with the pressure field at the solid surface. The right frame shows the velocity field in the liquid at a cross sectional plane through the bubble. With the spatial resolution of $\delta x_{\min }=1.8 \mu \mathrm{m}$ the numerical simulation predicts a fast-jet velocity of $732.2 \mathrm{~m} / \mathrm{s}$ at that instant of time.
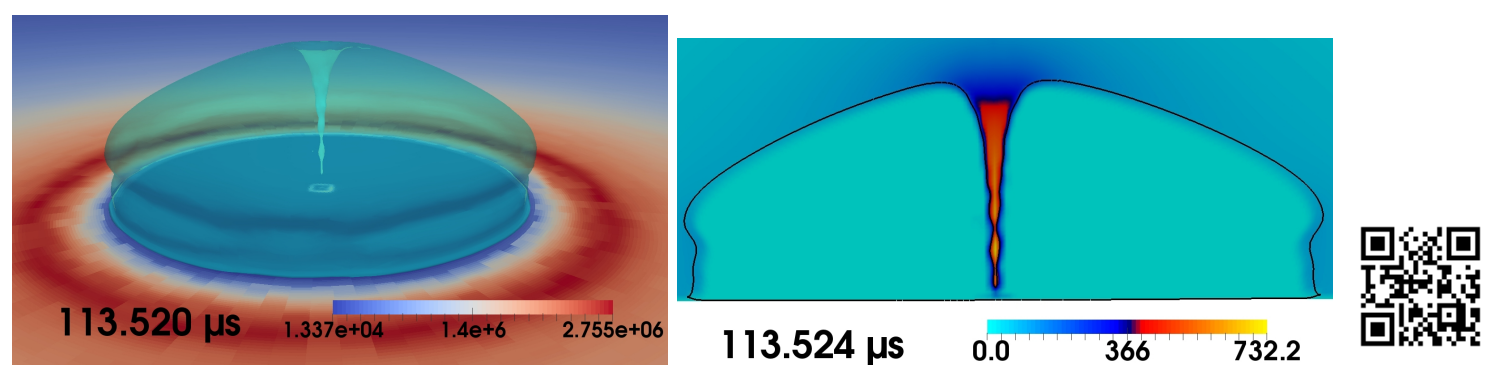

Fig. 5.5: Full 3D simulation of the fast-jet bubble. Left: contour plot of the bubble interface with the pressure in Pascal plotted in the plane of the solid boundary. Middle: Cross-section through the same bubble, plotted with the liquid velocity in $\mathrm{m} / \mathrm{s}$. Right: QR code to the URL https://doi.org/10.25625/1KJL3S of the movie of this simulation 
With the solution obtained, a pseudo-convergence study concerning the fast jet speed (Eq. B.2) was made using the state of the bubble at $t=109.864 \mu$ s as initial data for the following cases:

\begin{tabular}{l|llllr}
$\delta x_{\min }$ & $\mathrm{C}$ & $\mathrm{H}$ & $\mathrm{X}$ & $g_{f}$ & calculation time till \\
\hline \hline $1.6 \mu \mathrm{m}$ & $30 \mu \mathrm{m}$ & $160 \mu \mathrm{m}$ & $0.6 R_{\max }$ & 5 & $113.79 \mu \mathrm{s}: 30,826 \mathrm{~s} \approx 8.6 \mathrm{~h}$ \\
$1.7 \mu \mathrm{m}$ & $30 \mu \mathrm{m}$ & $160 \mu \mathrm{m}$ & $1.0 R_{\max }$ & 5 & $113.79 \mu \mathrm{s}: 33,333 \mathrm{~s} \approx 9.3 \mathrm{~h}$ \\
$1.8 \mu \mathrm{m}$ & $30 \mu \mathrm{m}$ & $160 \mu \mathrm{m}$ & $1.0 R_{\max }$ & 5 & $113.79 \mu \mathrm{s}: 22,959 \mathrm{~s} \approx 6.4 \mathrm{~h}$ \\
$1.9 \mu \mathrm{m}$ & $30 \mu \mathrm{m}$ & $160 \mu \mathrm{m}$ & $1.0 R_{\max }$ & 5 & $113.79 \mu \mathrm{s}: 19,427 \mathrm{~s} \approx 5.4 \mathrm{~h}$ \\
$2.0 \mu \mathrm{m}$ & $30 \mu \mathrm{m}$ & $160 \mu \mathrm{m}$ & $1.0 R_{\max }$ & 5 & $114.66 \mu \mathrm{s}: 41,931 \mathrm{~s} \approx 11.6 \mathrm{~h}$
\end{tabular}

Table 5.1: Parameters and calculation times with 16 threads on the Ryzen machine for the convergence study cases in Fig. 5.6.

More than just $\delta x_{\min }$ had to be varied in order to make computation times feasible. That is why only qualitative conclusions can be drawn. The original calculation accidentally included the sampling of the gas velocity for $v_{j}$ as well and is not included in the convergence study. None of the algorithms of Tab. 3.1 is applied, because the interpolated fields of ( $\left.p, \mathbf{U}, \alpha_{1}\right)$ of the original calculation at $t=109.864 \mu$ s were taken. The quantity $v_{j}$ in Fig. 5.6 is evaluated on the line defined by the endpoints $(0.1 \mu \mathrm{m}, 0,0)$ and $(0.1 \mu \mathrm{m}, 495 \mu \mathrm{m}, 0)$ with cell-mid-point interpolation. It can be seen that for all resolutions tested the maximum speed of the liquid during fast jetting exceeds $900 \mathrm{~m} / \mathrm{s}$. The fastest velocity of $\approx 1000 \mathrm{~m} / \mathrm{s}$ is observed for the resolution fitting the original one $(1.8 \mu \mathrm{m})$.
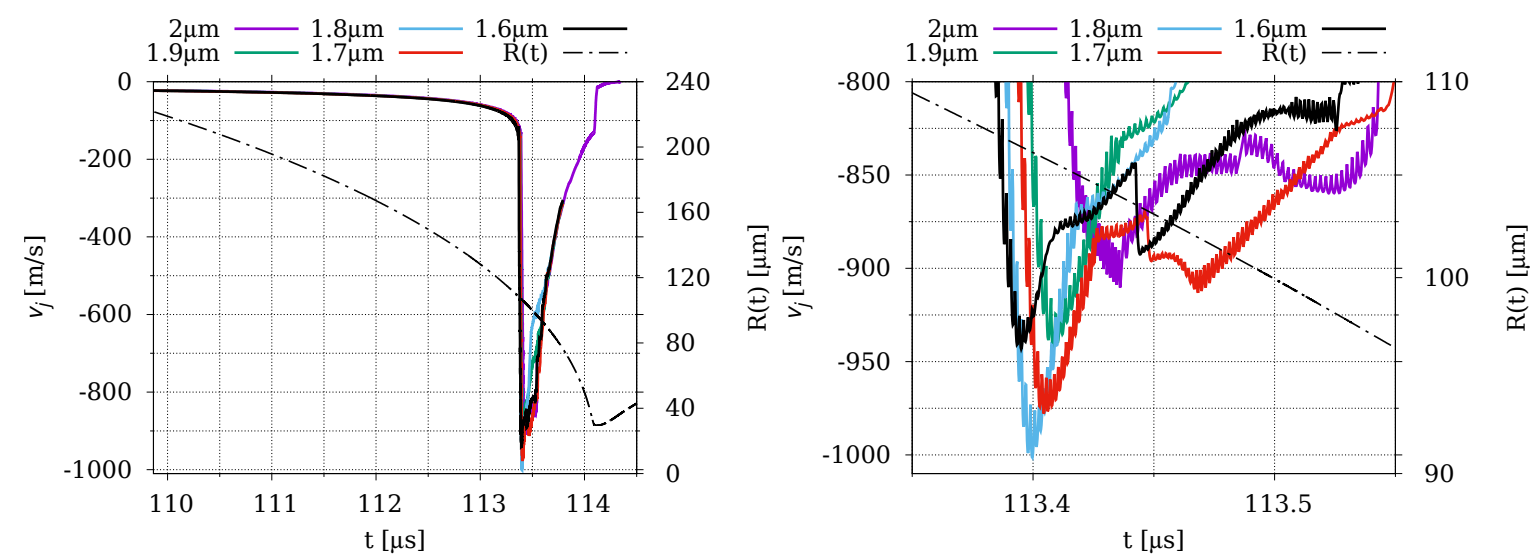

Fig. 5.6: Fast-jet speed $v_{j}$ (Eq. B.2) pseudo-convergence test with full 3D mesh 3.1.2.d, starting at $t=109.864 \mu \mathrm{s}$

\subsection{Quarter 3D simulation convergence study}

A quarter 3D simulation convergence study was done, too, starting at $t=0$ for all cases, because the amount of cells was much lower. The bubble has the same standard properties again:

$$
\begin{array}{r}
R_{\text {init }}=20 \mu \mathrm{m}, R_{n, 1}=184.1 \mu \mathrm{m}, R_{n, 2}=64 \mu \mathrm{m}, R_{\text {max,unbound }}=495 \mu \mathrm{m}, \\
R_{n} \text { reduction in interval }[30 \mu \mathrm{s}, 40 \mu \mathrm{s}], D_{\text {init }}=20.295 \mu \mathrm{m} .
\end{array}
$$

The Mesh 3.1.2.e is used with the geometric parameters described in the same Sec. 3.1.2.e. The initial data were set with the algorithm 0 from Tab. 3.1. Figure 5.7 shows the simulation with 
$\delta x_{\min }=1.8 \mu \mathrm{m}$ during jetting and the following Fig. 5.8 shows $v_{j}$ for three different resolutions including the $R(t)$ curve. Time stepping was the same as for the full 3D simulation.

When comparing Fig. 5.6 and Fig. 5.8, it is seen that the evolution is qualitatively similar but there are major differences in the details:

\begin{tabular}{l||r|r|r|r} 
& $2 T_{c} \approx$ & $2 T_{c}$ - time of jetting & $\min _{\delta x_{\min }}\left(\min _{t}\left(v_{j}\right)\right)$ & $\max _{\delta x_{\min }}\left(\min _{t}\left(v_{j}\right)\right)$ \\
\hline \hline full 3D & $114.1 \mu \mathrm{s}$ & $0.7 \mu \mathrm{s}$ & $-1001 \mathrm{~m} / \mathrm{s}$ & $-905 \mathrm{~m} / \mathrm{s}$ \\
\hline quarter 3D & $112.9 \mu \mathrm{s}$ & $0.35 \mu \mathrm{s}$ to $0.5 \mu \mathrm{s}$ & $-950 \mathrm{~m} / \mathrm{s}$ & $-678 \mathrm{~m} / \mathrm{s}$
\end{tabular}

Table 5.2: Comparing the results of Fig. 5.6 and Fig. 5.8.

Whether having had the far boundary at only $80 R_{\max }$ was the reason for non-convergence of the jet speed cannot be said. However, the same conclusion that was already stated in Lechner et al. (2020) can be drawn: Simulating the fast jet needs enormously high resolution.

The quest to find a more solid evidence for the fast jet existence was pursued on the experimental side again, but with Megaframes per second, as explained in the following paragraph.
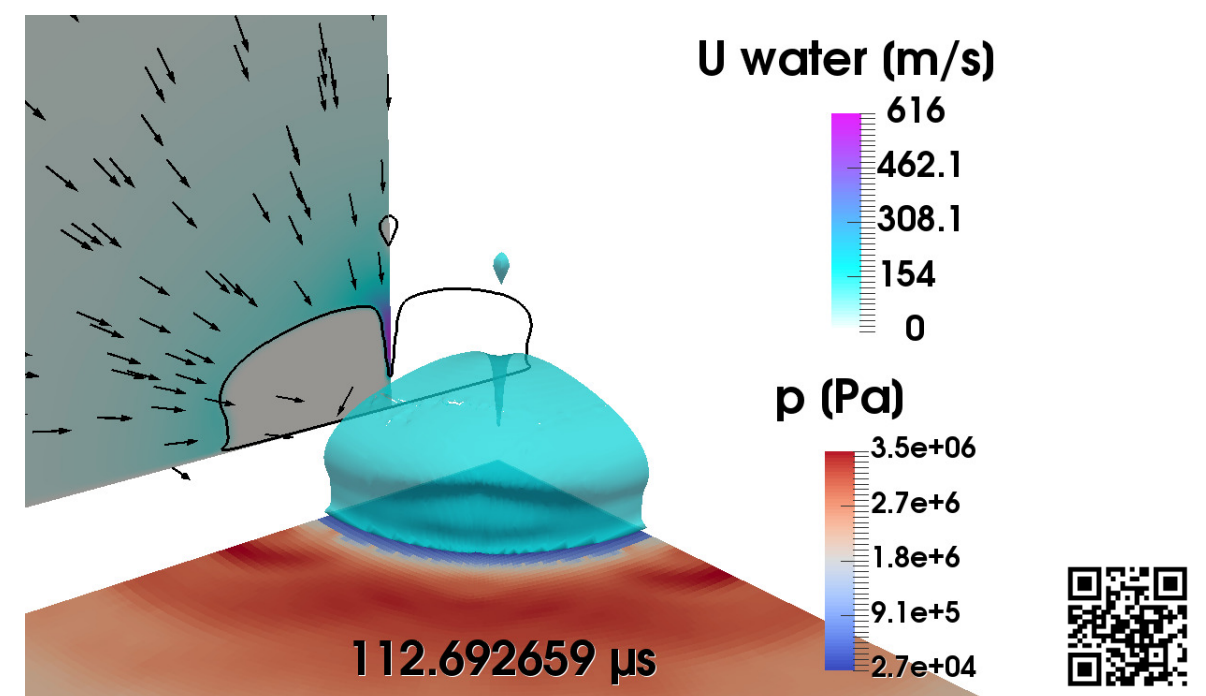

Fig. 5.7: Quarter 3D simulation of a bubble at $D^{*}=0.041$ during jetting with $\delta x_{\min }=1.8 \mu \mathrm{m}$. Pressure colored on the solid boundary, the liquid velocity given on a translated cross-section plane through the bubble including arrows for flow direction. Right: QR-code to the link for the movie of the simulation (https://doi.org/10.25625/KHO2SD). 


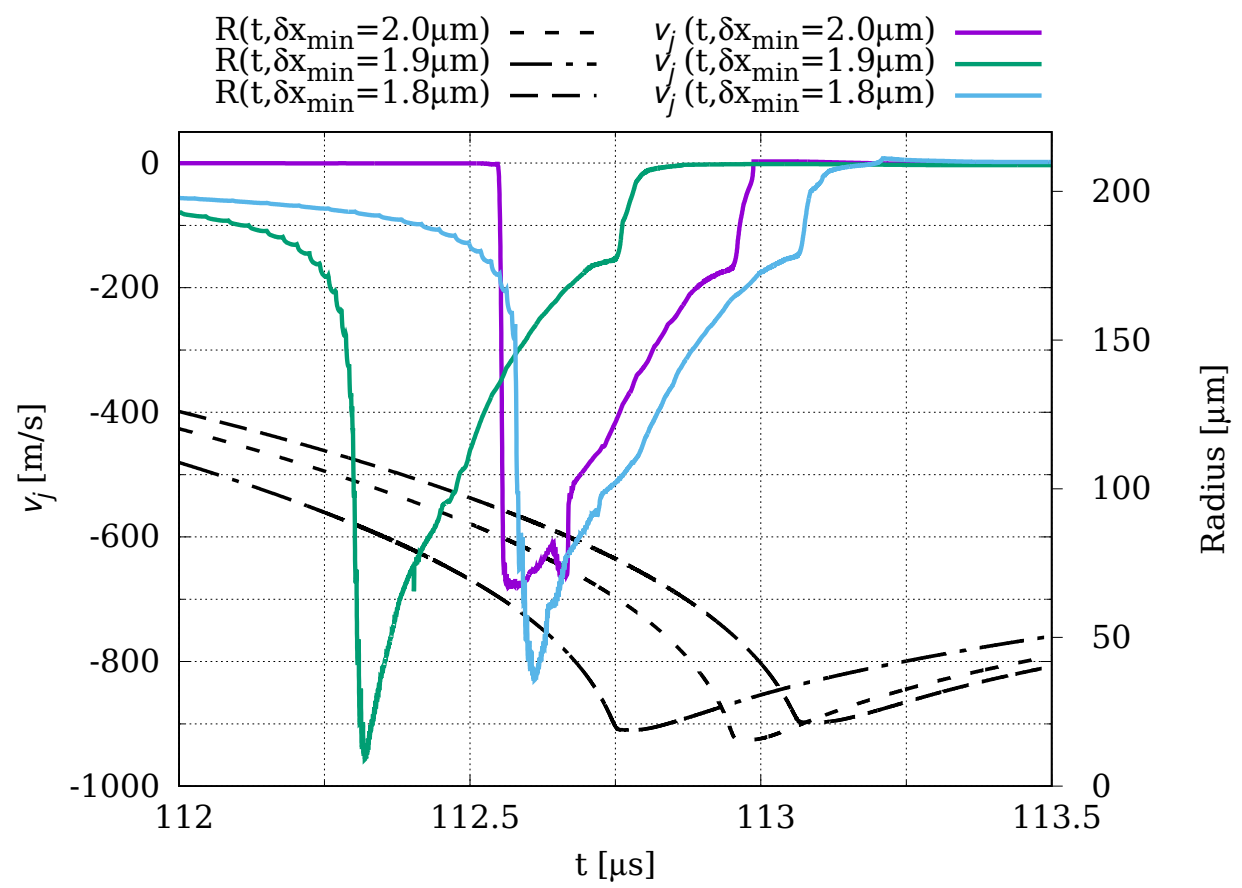

Fig. 5.8: Fast-jet speed convergence test with the quarter 3D Mesh 3.1.2.e

The cases ran for the following time on 30 threads on the Xeon-E5 machine:

\begin{tabular}{l|c}
$\delta x_{\min }$ & calculation time from $0 \mathrm{~s}$ till \\
\hline \hline $1.8 \mu \mathrm{m}$ & $150 \mu \mathrm{s}: 174,718 \mathrm{~s} \approx 48.5 \mathrm{~h}$ \\
$1.9 \mu \mathrm{m}$ & $140 \mu \mathrm{s}: 116,019 \mathrm{~s} \approx 32.2 \mathrm{~h}$ \\
$2.0 \mu \mathrm{m}$ & $140 \mu \mathrm{s}: 101,818 \mathrm{~s} \approx 28.3 \mathrm{~h}$
\end{tabular}

Table 5.3: Calculation times of the cases in Fig. 5.8

\subsection{Overlay with Mfps experiments}

Overlay with Megaframes per second experiments was performed, shown in Fig. 5.9. The odd rows show the photographs of the experiment with setup 2 (Sec. 3.3.2) and the even rows show the ray-tracing of a simulation in axial symmetry with the Mesh 3.1.2.a without the lower half, skipping the snappyHexMesh part. The geometric mesh parameters are the same as the parameters 5.1 for the simulation for the experiment with setup 1. The bubble parameters are:

$$
\begin{array}{r}
R_{\text {init }}=20 \mu \mathrm{m}, R_{n, 1}=142 \mu \mathrm{m}, R_{n, 2}=64 \mu \mathrm{m}, R_{\text {max,unbound }}=430 \mu \mathrm{m}, \\
R_{n} \text { reduction in interval }[60 \mu \mathrm{s}, 75 \mu \mathrm{s}], D_{\text {init }}=21.5 \mu \mathrm{m} .
\end{array}
$$

The value for $R_{\text {max,unbound }}$ is not necessarily the true one, but the mesh was created with this value. The bubble parameters were chosen such that the timing of the frame "(4) $80.0 \mu \mathrm{s}$ " fits to a good extent, so that the numerical times can be regarded as an estimate of the time resolution thus gained.

The frames again are rotated so that the glass surface boundary is located in the lower part and the laser entered from the top of the frames. The first frame shows the plasma of the laserinduced breakdown, and from the second frame onward the collapse phase of the bubble is shown. The sequence is a montage of 7 measurements in total, the measurement number is 


\section{The quest for the fast jet}

denoted by the number in brackets in each experimental frame heading. The exposure time was the same for all measurements $(150 \mathrm{~ns})$ while the inter-frame time varied from $1 \mu$ s to $350 \mathrm{~ns}$. By stacking/ interleaving the single frames of the reproducible measurements together, a time resolution down to $34 \mathrm{~ns} \approx 29.4 \mathrm{Mfps}$ could be achieved in the time of jetting. Here, the times of the simulation are taken to indicate the time progression.

The typical bell shape is seen, which was already reported in Benjamin and Ellis (1966) and predicted in Lechner et al. (2019). The ray-tracing shows most light and shadow features of the experiment. The bubble is magnified, so that it can be seen that the bubble was indeed pierced. However, the viewing perspective permits only a slim glimpse into the bubble, so it is not suitable for speed detection.

To sum up, the investigations concerning the fast jet together prove that a) the bubble shape including the fast jet is not an artefact of axial symmetry, but a full 3D feature and $b$ ) the bubble shape is well captured by the CFD simulations. This shows that the overlay-method multiplies scientific interpretation possibilities for (two-phase) fluid flows, even reaching out to hardly measurable phenomena. Taking these arguments together, it is most likely that there was a fast jet occurring also in the real experiment. Apparently, the slim window to look through the bubble at this viewing perspective makes the possibility likely to miss it. The simulation shows that there is a window of $96 \mathrm{~ns}$ to film it and determine a velocity. 


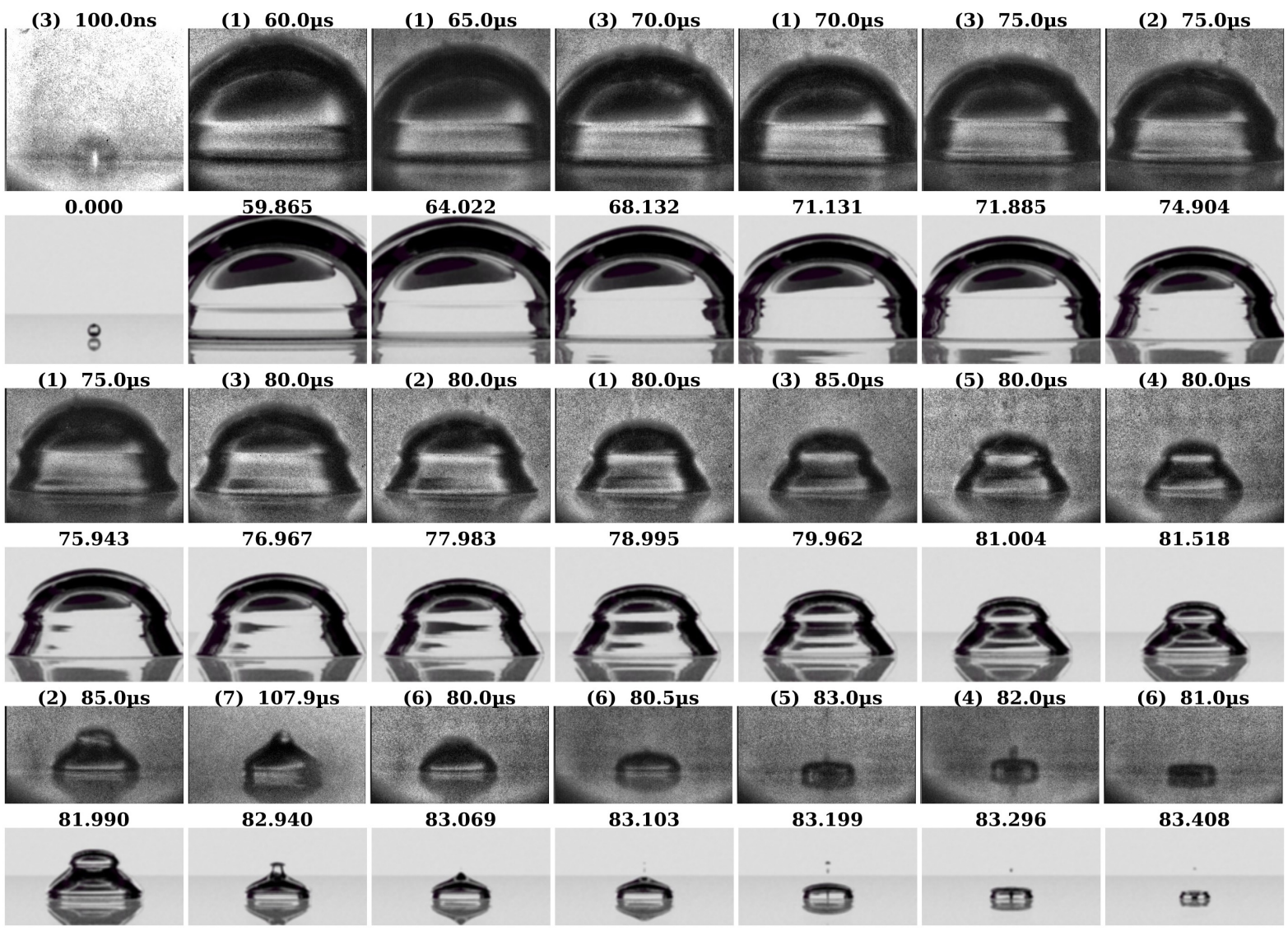

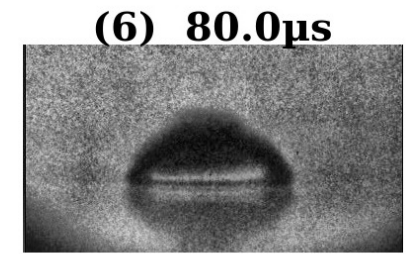

83.069

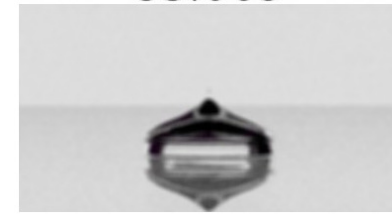

Fig. 5.9: Top: Bubble generated directly at the solid boundary. Odd rows are experimental recordings with measurement number denoted in brackets, even rows are ray-tracing images of the numerical simulation in Fig. 5.10. Exposure time of the experimental frames is $150 \mathrm{~ns}$, except for the first frame with the plasma, which has an exposure time of $500 \mathrm{~ns}$ and is enhanced in contrast. The width of the frames is $664.5 \mu \mathrm{m}$. Bottom: magnification 

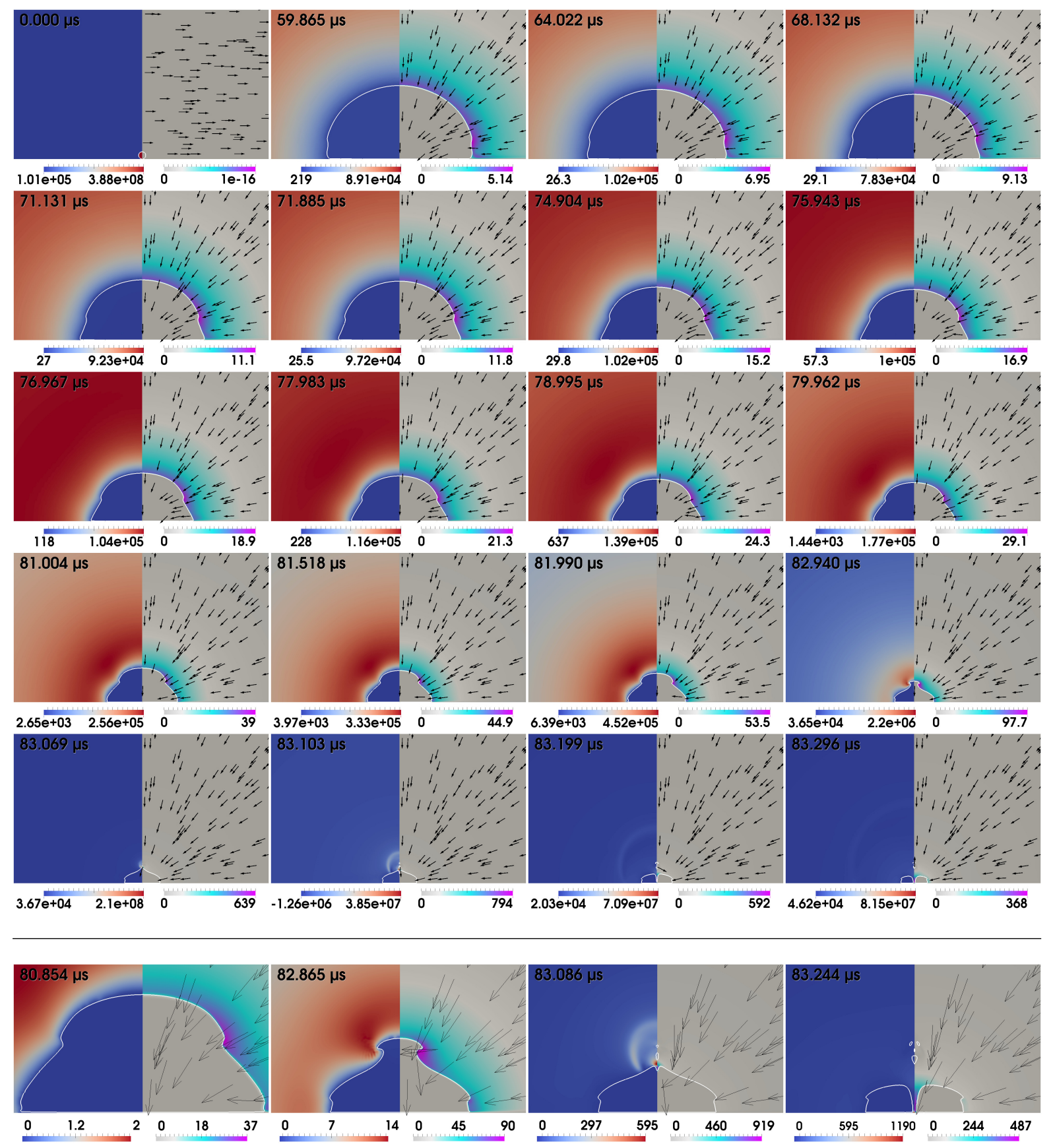

Fig. 5.10: Simulation of a $D^{*} \approx 0$ bubble in axial symmetry. Top: bubble expansion and collapse at time instants fitting the images from the experiment in Fig. 5.9. Left side of the frames is pressure in pascal, right side liquid velocity in $\mathrm{m} / \mathrm{s}$. Bottom: zoom into the time of jetting. Left is pressure in bar, right liquid velocity in $\mathrm{m} / \mathrm{s}$ 


\section{Jet velocity, pressure on the solid boundary and maximum radius, dependent on $D^{*}$}

\subsection{Jet velocity}

This section summarizes the jet velocities of a bubble close to the solid boundary found in Philipp and Lauterborn (1998), Lechner et al. (2020) and in this thesis. The jet speed in the range $D^{*} \in[0 ; 3]$ is investigated. The values found are given in Fig. 6.4 and the values of this thesis are plotted into the same diagram. It has to be noted that the code C. Lechner uses and the code used in this thesis don't share the same syntax because both authors maintained their own versions of code and only compared the results. The jet-speed is defined in Lechner et al. (2020) as:

In the case of the standard axial jet by involution of the top of the bubble the velocity of the jet tip is taken shortly before impact onto the opposite bubble wall. That is, because already shortly before impact the gas in the gap decelerates the jet by compression. As to the fast jet, the definition has been adopted to take the average velocity from annular jet collision up to jet impact. This quantity is unambiguously defined.

These definitions are adopted here. The times have been found by analyzing the bubble shape with Paraview, which limits the precision to the hard-disk write interval of 30 timesteps. This, however, is still precise enough because of the adaptive time step size. The timings are indicated by black dotted lines in Figs. 6.2 for the microjet of the calculations from Ch. 4 and in Fig. 6.3 for one $D^{*}=0$ example in axial symmetry for the fast jet. The time-averaged velocity for the fast jet was found by integrating $\min \left(\mathbf{U}_{y}\right)$ with the trapezoid rule between the two time marks and then divide by the time full interval length.

The experimental data from Philipp and Lauterborn (1998) are given in terms of $\gamma_{d}$ :

$$
\gamma_{d}=D_{\text {init }} / R_{\max }
$$

with $R_{\max }$ taken from the measurement. From the numerical data both $\gamma_{d}$ and $D^{*}$ can be obtained. An almost perfect linear correlation is found, shown in Fig. 6.1:

$$
D^{*}=0.9798 \gamma_{d}-0.0073
$$

If the data points from Philipp and Lauterborn (1998) are modified considering the above relation, a slightly better agreement with the numerical data in Fig. 6.4 is apparent. However, it is seen that experimental and numerical data do not fit over the whole spectrum of $D^{*}$. Three assumptions are investigated why this may be the case. The first assumption (I) is that there are 
refraction issues deviating the true value of jet velocity. The authors assumed a linear distortion of the position of the jet tip inside the bubble due to refraction. As stated in Sec. 4.2, there may be more complex distortions involved. For a spherical bubble, however, the distortion might not be as dramatic. The authors stated that they multiplied the jet speed visually obtained by a factor of 1.33. This factor can be verified again by measuring the distances of the rays in Fig. $1.2 \mathrm{~b}$ to the optical axis i) to the left of the bubble and ii) in the middle. This yields a factor of $1.372 \pm 0.014$ when fitting the distances with a simple linear function. To be sure, the simulation of the bubble for the data point at $D^{*}=3$ is rendered with blender and the Fig. 6.5 is obtained.

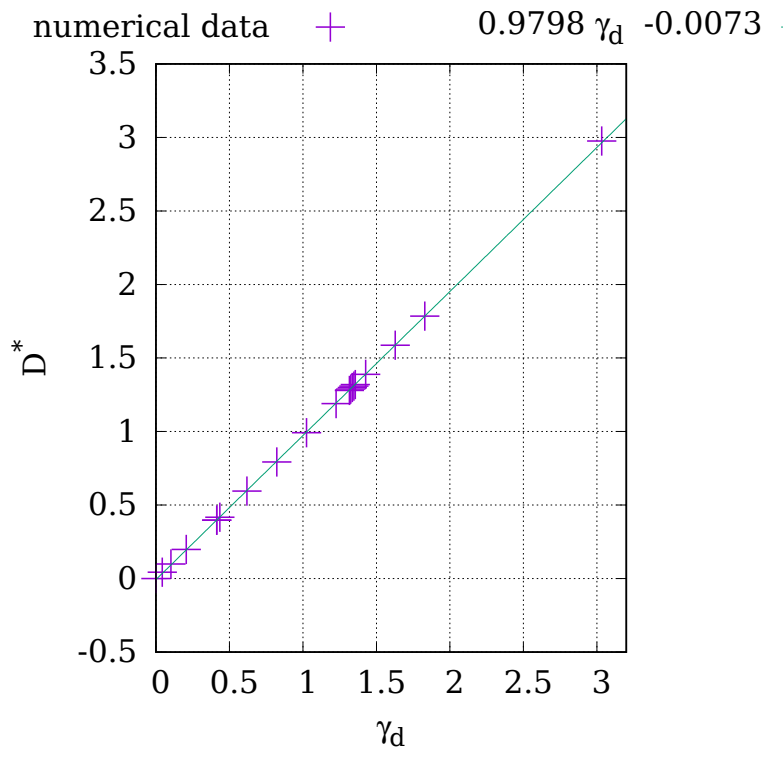

Fig. 6.1: Correlation of $D^{*}$ and $\gamma_{d}$ if $\gamma_{d}$ is defined as in Eq. 6.1.

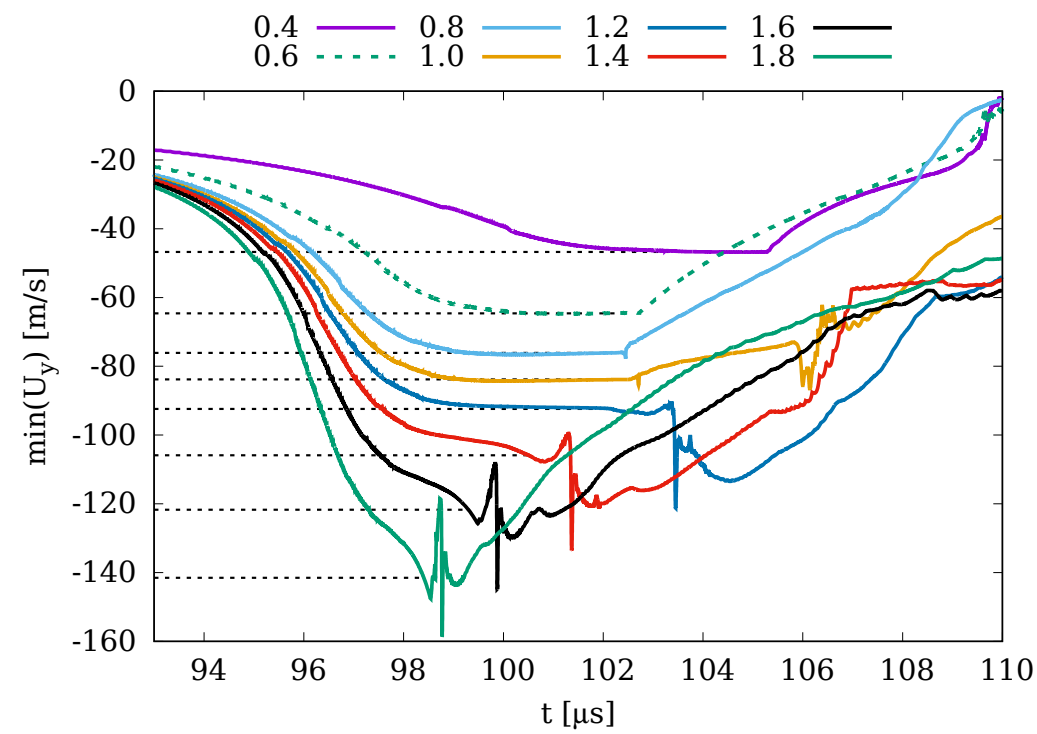

Fig. 6.2: Jet speed over time for the simulations where a microjet occurs. The black dotted lines indicate the location of jet speed value reading according to the definition in Lechner et al. (2020). $D^{*}=3$ not shown here for the sake of visibility of the other values. 
6 Jet velocity, pressure on the solid boundary and maximum radius, dependent on $D^{*}$

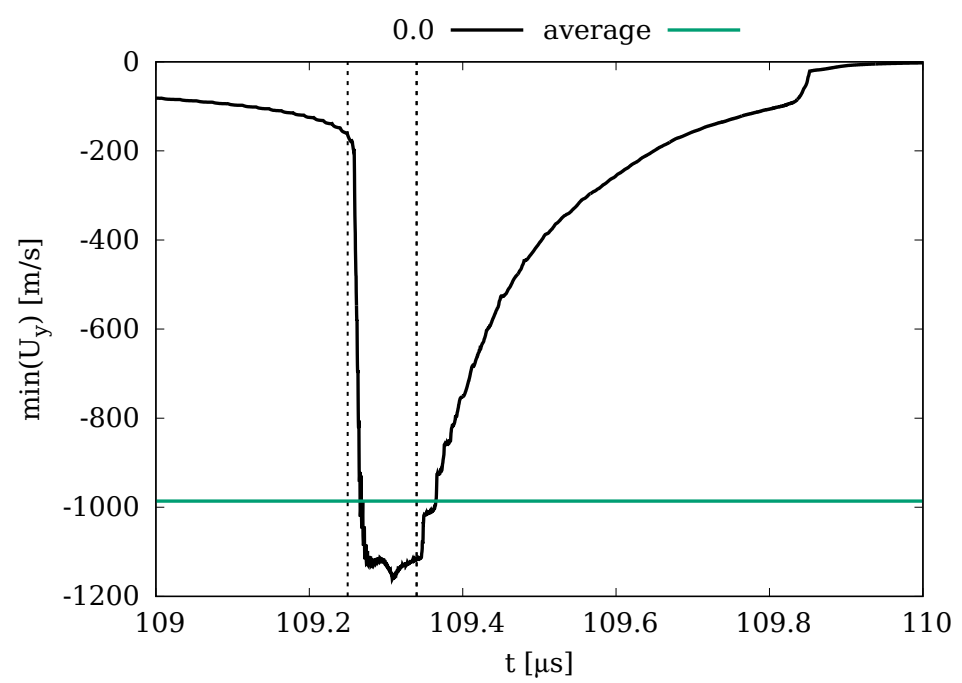

Fig. 6.3: Jet speed over time for the simulation at $D^{*}=0$. Black dotted lines indicate the time interval for averaging and the green line indicates the averaging result.

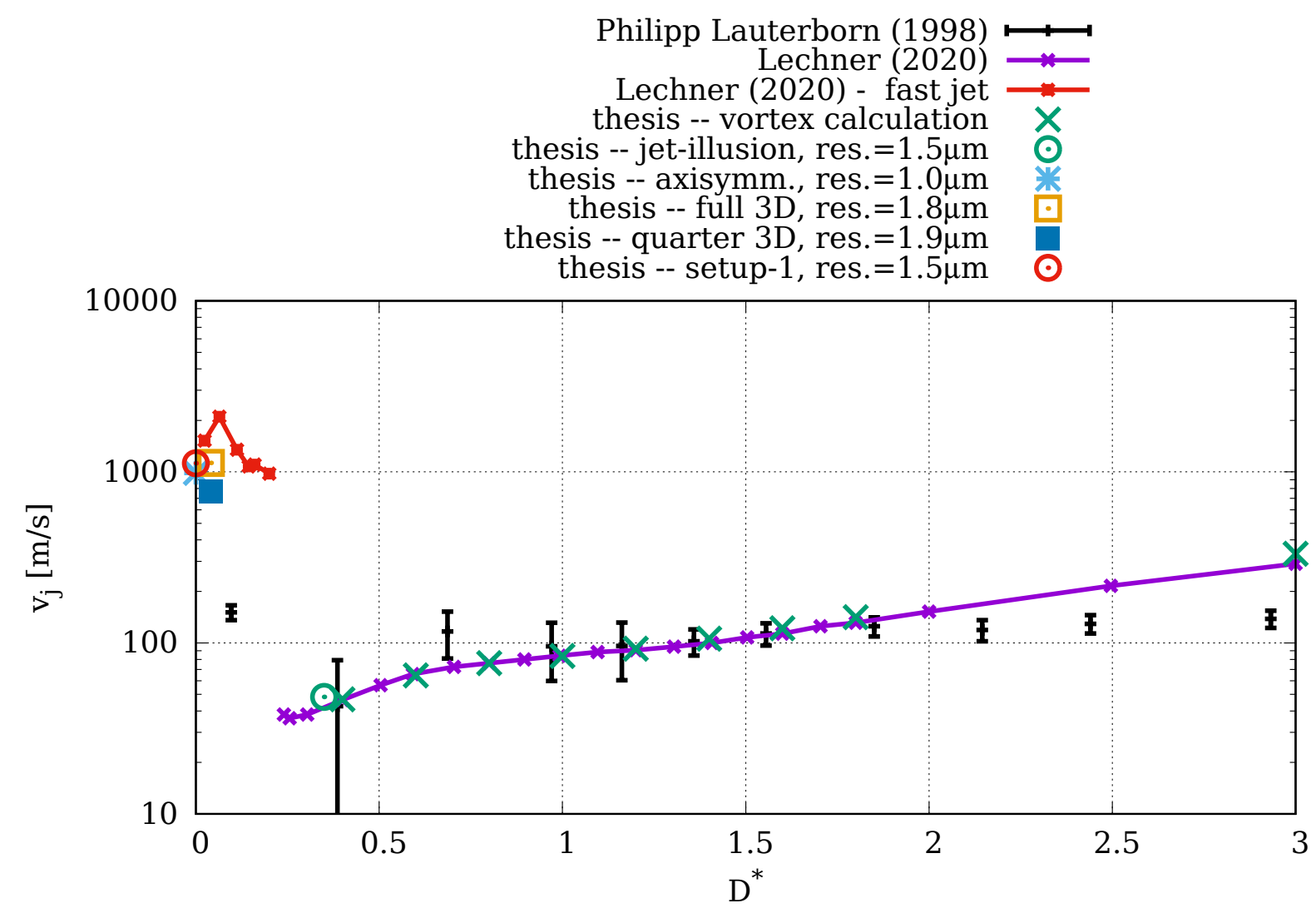

Fig. 6.4: Jet speeds obtained in Lechner et al. (2020) with the values of this thesis and those of Philipp and Lauterborn (1998) plotted in the same diagram. For deviation of the experimental data of Philipp and Lauterborn (1998) and the numerical one, see text. Jet-illusion refers to the simulation of Fig. 4.18, axisymm. $1 \mu \mathrm{m}$ refers to Fig. 5.10, full $3 D$ to Fig. 5.5, quarter $3 D$ to Fig. 5.8 and setup- 1 to Fig. 5.1 


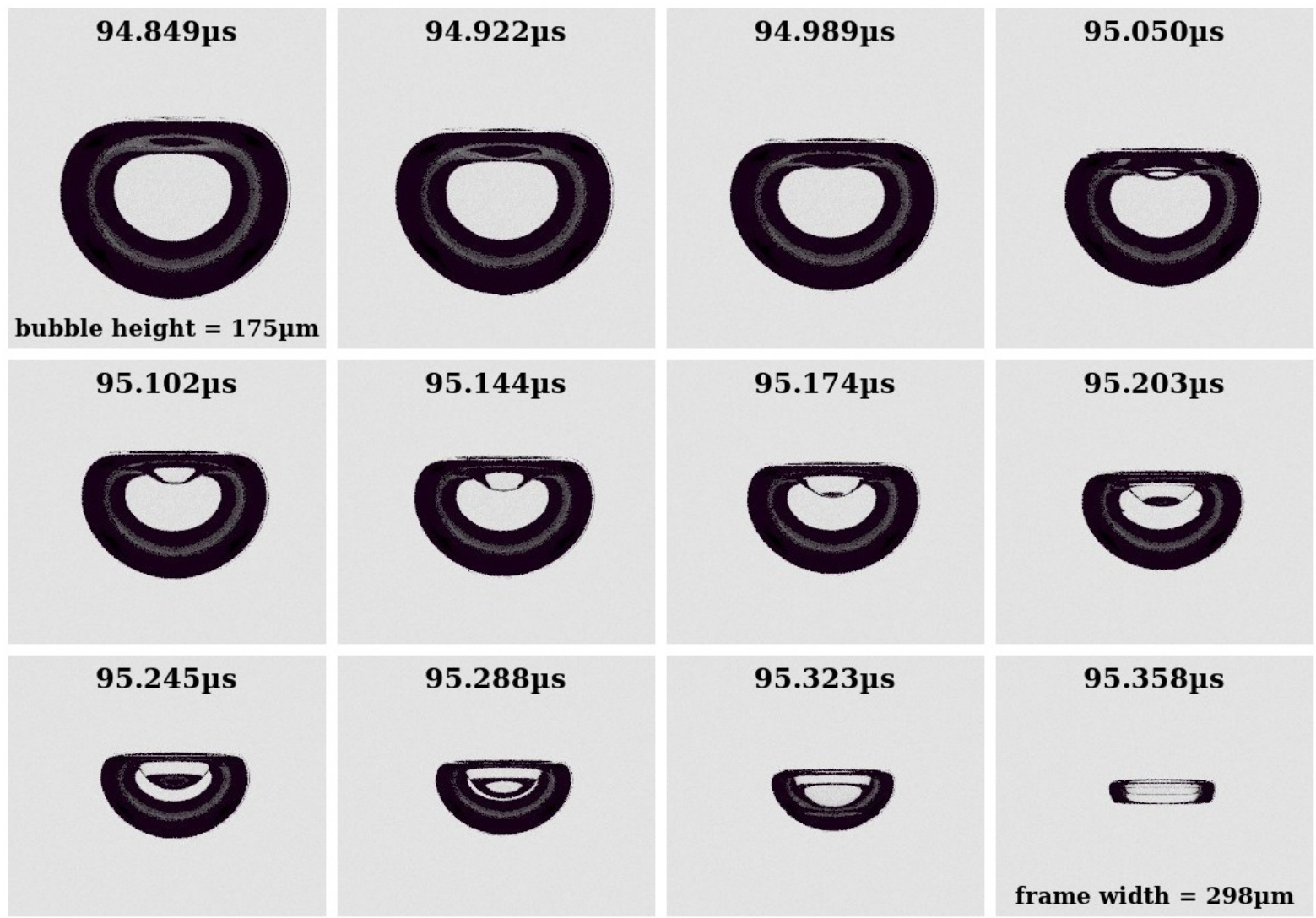

Fig. 6.5: Rendered results of the calculation for $D^{*}=3$, used to show the much too low jet speed value obtained by simple observation.

With the sequence in Fig. 6.5 the same procedure as would be done with experimental data is applied. The traveling distance of the jet tip is taken and multiplied by 1.33. In Fig. 6.6 the scientific representation of the numerical simulation is given for the same time instants. Tab. 6.1 summarizes the different measures one would obtain if only two of the rendered frames were available, compared to the average jet speed obtained by the scientific representation. It turns out that in this way, the pseudo-experimental data even slightly over-estimates the jet speed.

\begin{tabular}{l|r|r} 
frames & $\begin{array}{r}1998 \\
\text { approach } \\
\text { (incl. 1.33) }\end{array}$ & $\begin{array}{r}\text { numerical } \\
\text { average }\end{array}$ \\
\hline \hline 4 to 8 & $260 \mathrm{~m} / \mathrm{s}$ & $247 \mathrm{~m} / \mathrm{s}$ \\
5 to 8 & $295 \mathrm{~m} / \mathrm{s}$ & $252 \mathrm{~m} / \mathrm{s}$ \\
6 to 8 & $337 \mathrm{~m} / \mathrm{s}$ & $258 \mathrm{~m} / \mathrm{s}$ \\
7 to 8 & $392 \mathrm{~m} / \mathrm{s}$ & $261 \mathrm{~m} / \mathrm{s}$ \\
6 to 9 & $337 \mathrm{~m} / \mathrm{s}$ & $267 \mathrm{~m} / \mathrm{s}$ \\
9 to 10 & $231 \mathrm{~m} / \mathrm{s}$ & $296 \mathrm{~m} / \mathrm{s}$
\end{tabular}

Table 6.1: Evaluation of the jet speeds from Figs. 6.5 and 6.6. 


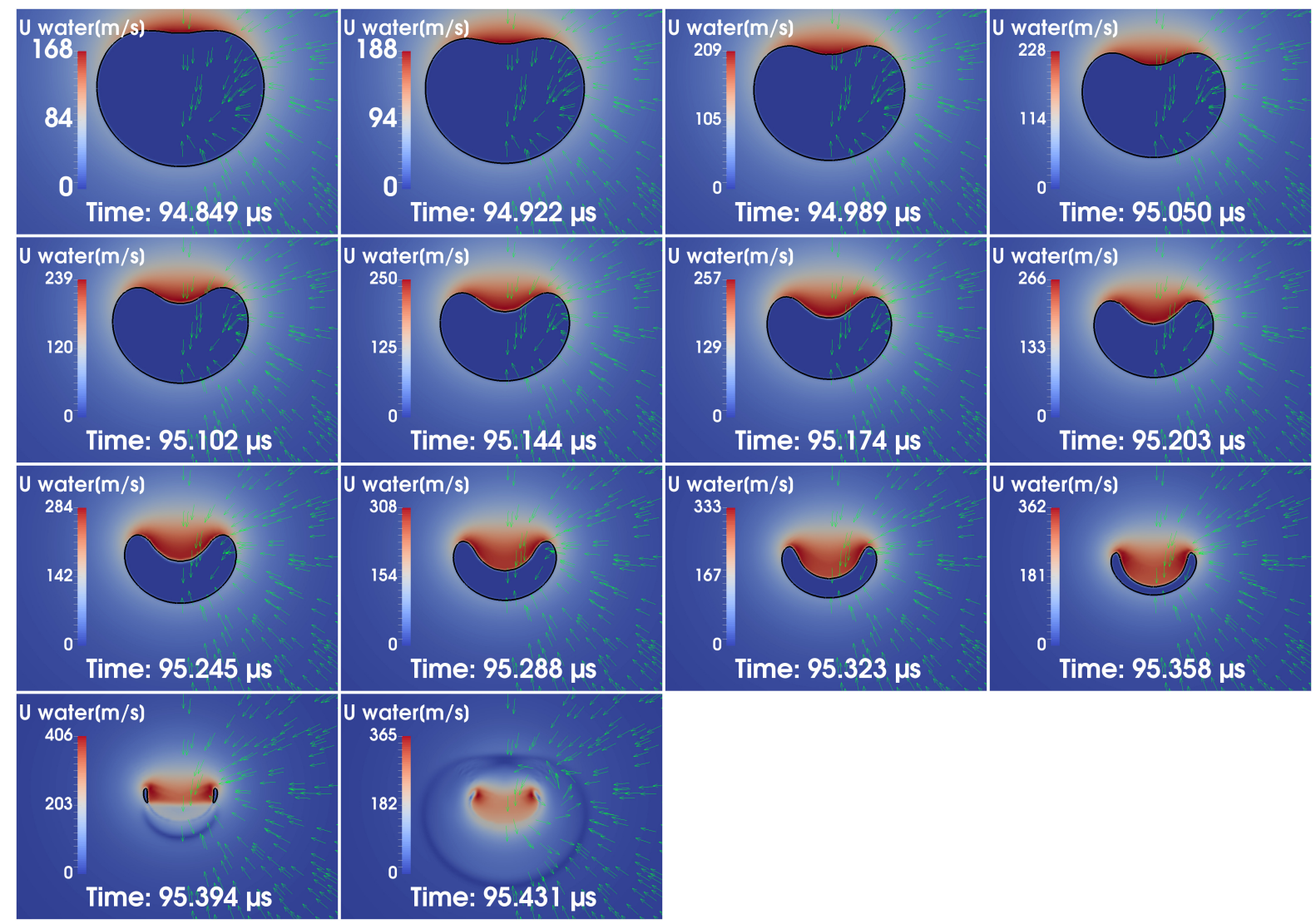

Fig. 6.6: Velocity field of the water of the underlying simulation of Fig. 6.5.

Another issue not taken into account here is that the jet speed is given as absolute value in Fig. 6.4, while the distance to the solid boundary is given in normalized values. The second assumption (II) is that a bubble of $1.45 \mathrm{~mm}$ size (Philipp and Lauterborn 1998) has different jet speeds than a $499.08 \mu \mathrm{m}$ bubble as investigated in the numerical case. In order to clarify this, the simulation for $D^{*}=3$ has been repeated for a bubble with $R_{\max }=1464 \mu \mathrm{m}$, yielding however a very similar result (see Fig. 6.7).

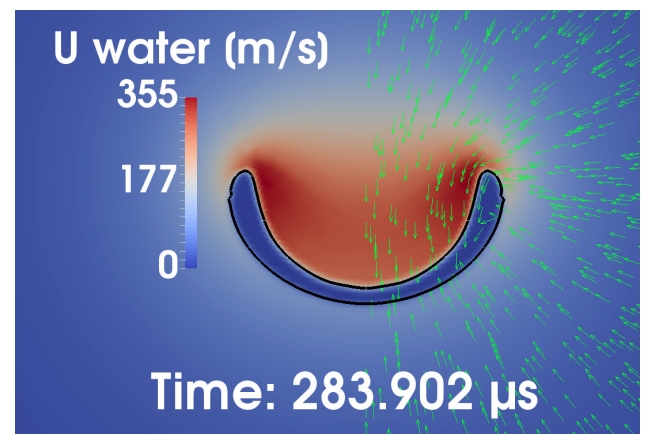

Fig. 6.7: Velocity field of a bubble at $D^{*}=3$ shortly before jet impact, $R_{\max }=1464 \mu \mathrm{m}$.

The only conclusion is that Philipp and Lauterborn (1998) underestimated their errors in the higher $\gamma_{d}$ data points. Their frame interval was $17.7 \mu$ s and especially in the measurement for $\gamma_{d}=3$ the time interval from onset of jet formation till jet impact is photographically missed. In the measurement for $\gamma_{d}=2.5$ only one frame with a jet is seen shortly before impact. 
6 Jet velocity, pressure on the solid boundary and maximum radius, dependent on $D^{*}$

\subsection{Maximum radius}

The values for $R_{\max }\left(D^{*}\right)$ were extracted from the calculations shown in the Figs. 6.2, 6.3 and 6.6. The normalized data $R_{\max }\left(D^{*}\right) / R_{\text {max,unbound }}$ is plot into Fig. 6.8

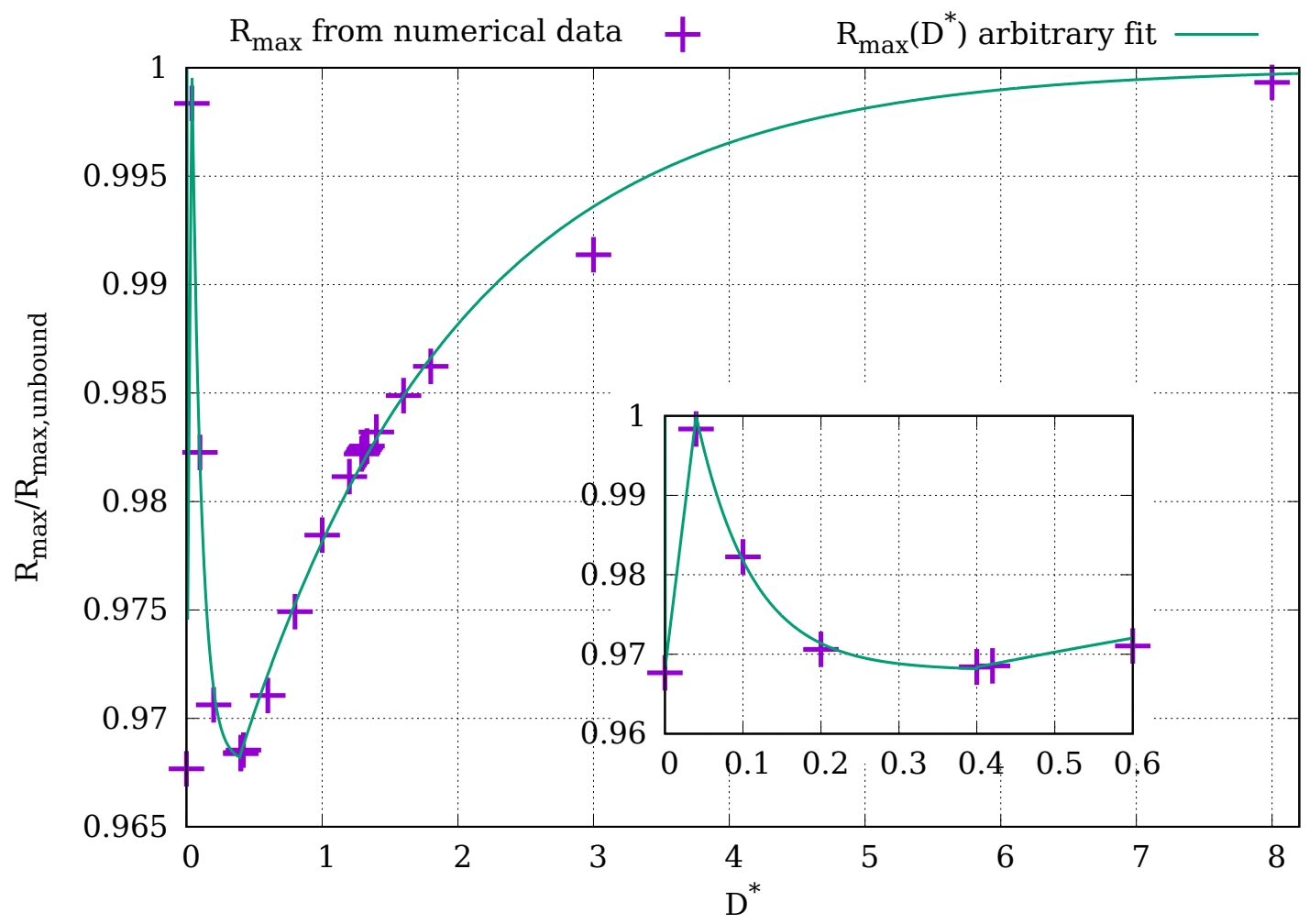

Fig. 6.8: Fitting Eq. 6.3 to the data of the normalized maximum radius over $D^{*}$.

$$
\begin{aligned}
k\left(D^{*}\right) & =1-(1-a) \cdot \exp \left(-b D^{*}\right) \\
l\left(D^{*}\right) & =c+(1-c) \cdot \exp \left(-d\left(D^{*}-0.04\right)\right) \\
m\left(D^{*}\right) & =0.968+\frac{1-0.968}{0.04} \cdot D^{*} \\
R_{\max }\left(D^{*}\right) / R_{\text {max,unbound }} & = \begin{cases}m\left(D^{*}\right) & \forall D^{*} \in[0,0.04] \\
l\left(D^{*}\right) & \forall D^{*} \in(0.04,0.4] \\
m\left(D^{*}\right) & \forall D^{*} \in(0.4, \infty)\end{cases} \\
a=0.95958 \pm 0.00071, b & =0.614 \pm 0.018, c=0.96798 \pm 0.00080, d=14.0 \pm 1.4
\end{aligned}
$$

The way the data points are arranged, ironically reminds of a Lennard-Jones potential function. However, the curvatures of a Lennard-Jones function do not fit well, which is why the step-wise arbitrary function 6.3 was defined. For values of $D^{*}<0.042$ the initial spherical bubble of $R_{\text {init }}=20 \mu \mathrm{m}$ is cut by the solid boundary. For the data point $D^{*}=0$ algorithm 2 of Tab. 3.1 accounted for adjusting the pressure even when bubble volume is lost. However, a jump in $R_{\max }\left(D^{*}\right) / R_{\max , \text { unbound }}$ is to be expected. 


\subsection{Pressure on the solid boundary}

In Sec. 2.3 the erosion potential was theoretically correlated with the yield stress of the material of several hundred Megapascal. In this section, the axisymmetric simulations of this thesis are evaluated concerning their pressure signal on the solid boundary in the center below the bubble (at the axis of symmetry). Again, the values for the bubbles are:

$$
R_{\text {init }}=20 \mu \mathrm{m}, R_{n, 1}=184.1 \mu \mathrm{m}, R_{n, 2}=64 \mu \mathrm{m}, R_{n} \text { reduction in interval }[60 \mu \mathrm{s}, 75 \mu \mathrm{s}] \text {, }
$$

and the Mesh 3.1.2.a is used in combination with snappyHexMesh to induce a cut at $D_{\text {init }}$ below the bubble. The time resolution is equal to the time step size. Figures 6.9 and 6.10 show the pressure signal in the time domain of the first collapse. The main stress happens during this time, the rebounds can be neglected. The shockwave of the bubble formation is disregarded here, too, because for the main erosive $D^{*}$ values its peak value was less than one third of the collapse shockwave.
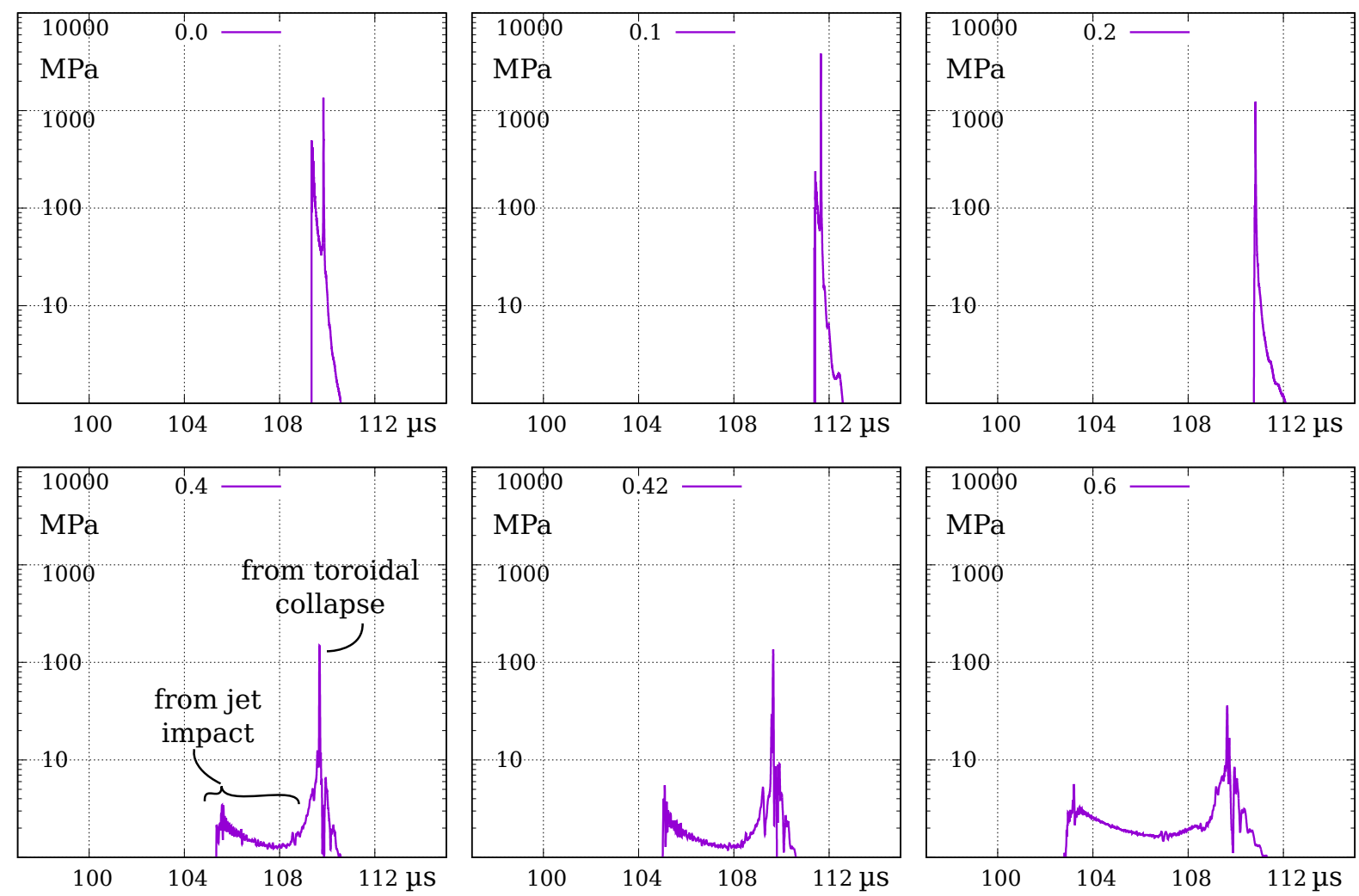

Fig. 6.9: Pressure in Megapascal (MPa) at the the solid boundary in the symmetry point below the bubble over time in Microseconds for the lower values of $D^{*}(0$ to 0.6$)$, evaluated during the collapse time domain. 
6 Jet velocity, pressure on the solid boundary and maximum radius, dependent on $D^{*}$
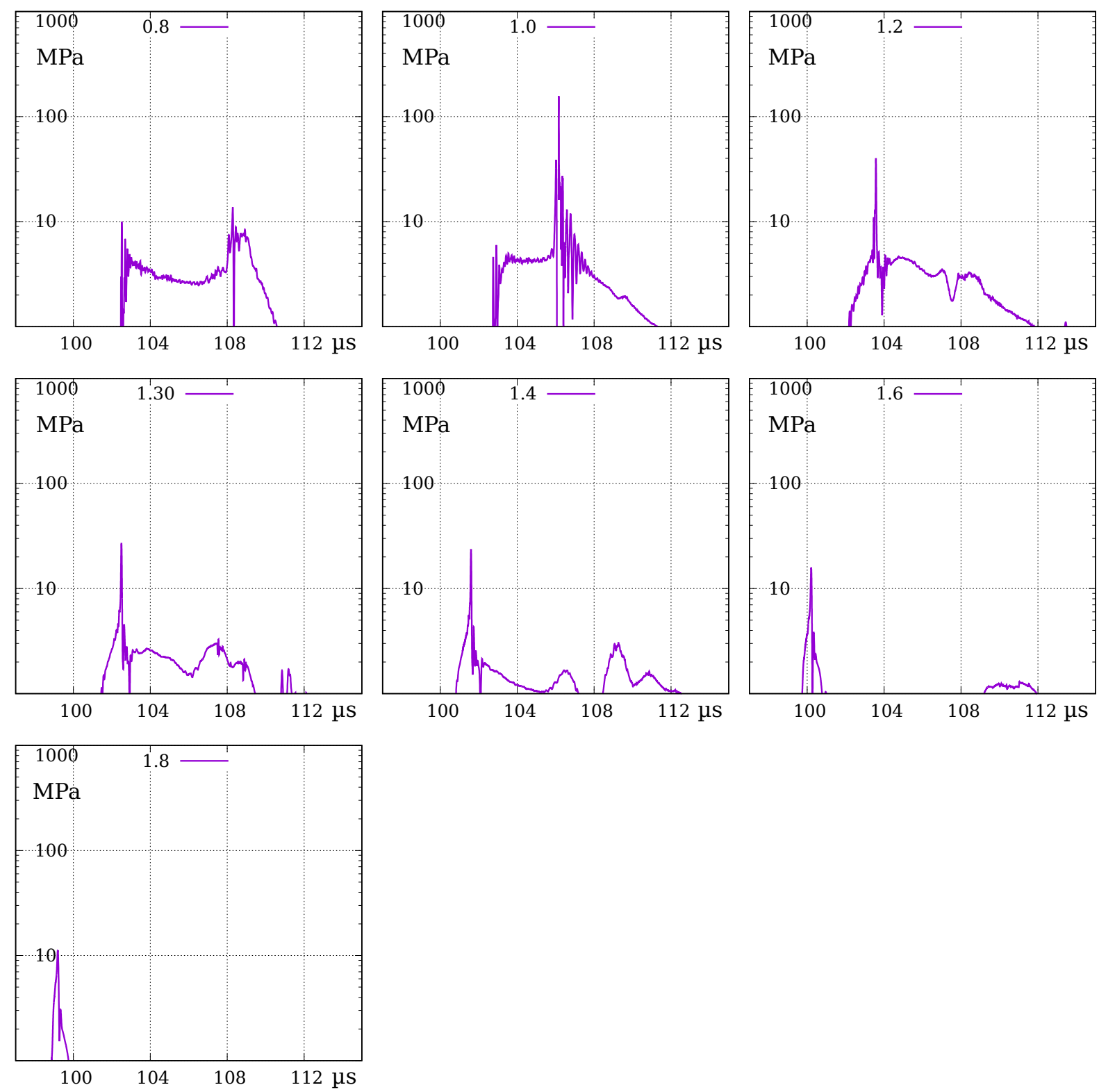

Fig. 6.10: Pressure in Megapascal (MPa) at the the solid boundary in the symmetry point below the bubble over time in Microseconds for the higher values of $D^{*}(0.8$ to 1.8), evaluated during the collapse time domain.

The transition can be seen from strong shockwave focusing due to toroidal collapse at the solid boundary for lower values of $D^{*}$ (Fig. 6.9) towards higher emphasis on the jet impact for medium values of $D^{*}$ and lastly a decay of erosive potential for higher values of $D^{*}$ (Fig. 6.10).

The curves have been processed to get the average stress on the solid boundary, as well as the peak stress. The average has been found the following way:

$$
p_{\text {avg }}=\frac{\int \varpi p \mathrm{~d} t}{\int \varpi \mathrm{d} t}, \quad \varpi=\left\{\begin{array}{ll}
0 & \forall p<1 \mathrm{MPa} \\
1 & \forall p \geq 1 \mathrm{MPa}
\end{array} .\right.
$$

The results are given in the two diagrams of Fig. 6.11 . 

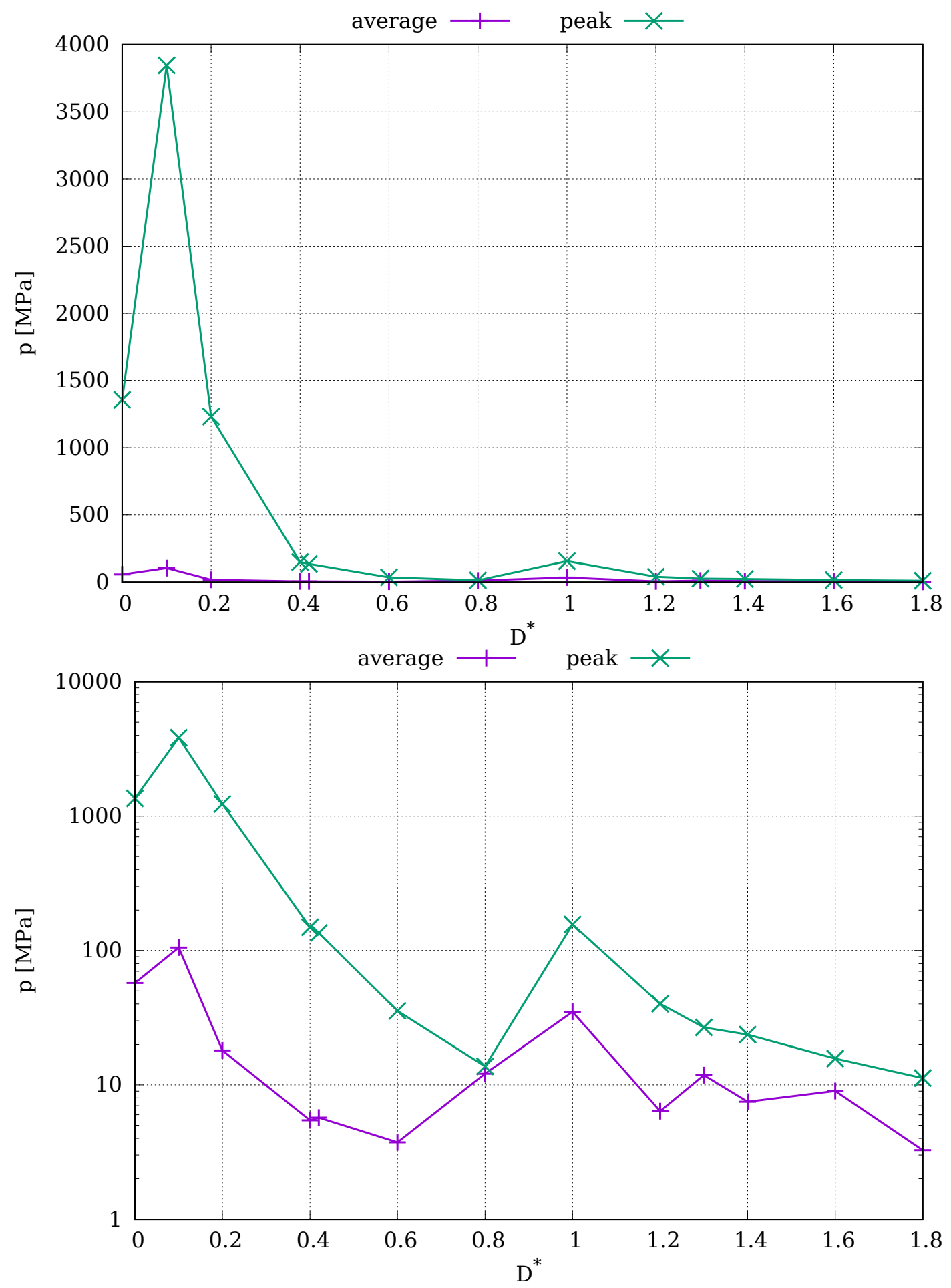

Fig. 6.11: Peak and average stresses in Megapascal over $D^{*}$, evaluated from Figs. 6.9 and 6.10. Linear in top diagram and logarithmic in bottom diagram.

From these plots it can be deduced that it is most likely that the main erosion happens around $D^{*}=0.1$, where peak values of close to $4 \mathrm{GPa}$ are observed. A first evaluation for the reason of this pressure peak is given in Fig. 6.12 for $D^{*}=0.1$. The fast jet has already impacted onto the solid boundary before the main pressure peak at $111.65 \mu$ s, contributing to a stagnation point pressure signal. The main peak roots from the focusing of the toroidal shockwave from the first collapse of the bubble directly on the solid boundary. To conclude, it can be said that one single, 
6 Jet velocity, pressure on the solid boundary and maximum radius, dependent on $D^{*}$

laser generated bubble of $R_{\max } \approx 500 \mu \mathrm{m}$ can erode any alloy when generated at a distance of $D^{*}=0.1$.

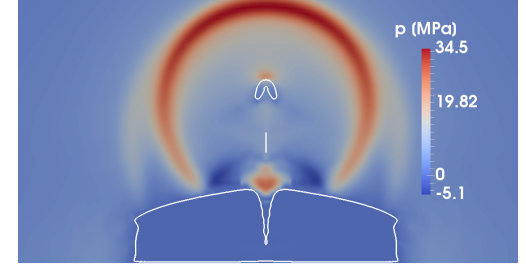

Time: $111.373 \mu \mathrm{s}$

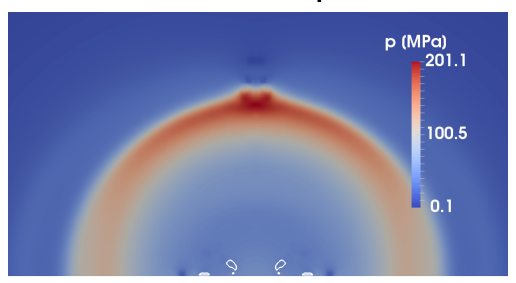

Time: $111.705 \mu \mathrm{s}$

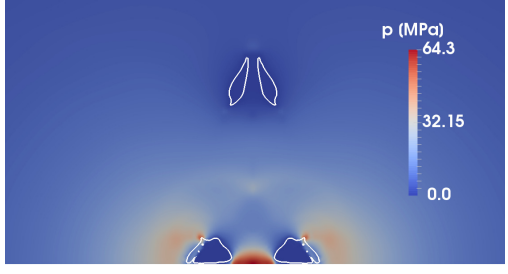

Time: $111.608 \mu \mathrm{s}$

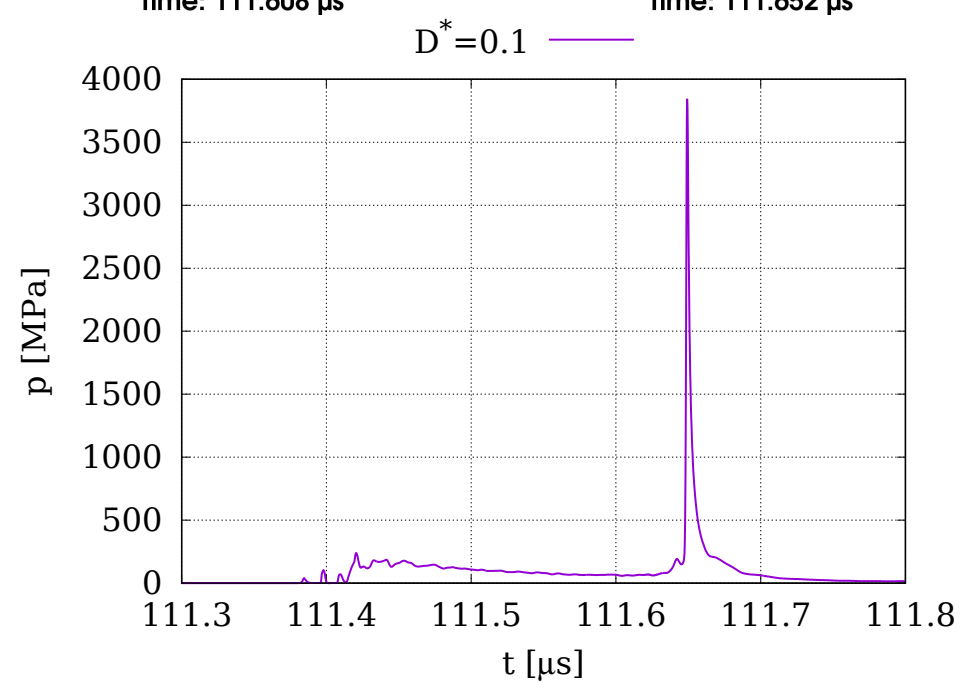

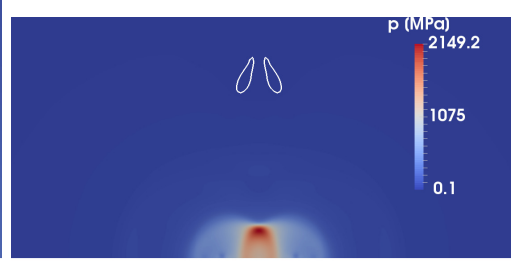

Time: $111.652 \mu \mathrm{s}$

Fig. 6.12: Temporal evolution of the pressure field around and after fast-jet impact for a bubble at $D^{*}=0.1$. Simulation done in axial symmetry. The diagram shows the pressure signal in the symmetry point below the bubble at the solid boundary. It is identical to the one in Fig. 6.9, but with a linear $y$-axis. The pressure peak roots from shockwave focusing. 


\section{Bubble dynamics close to a rigid cylinder - mushroom shape}

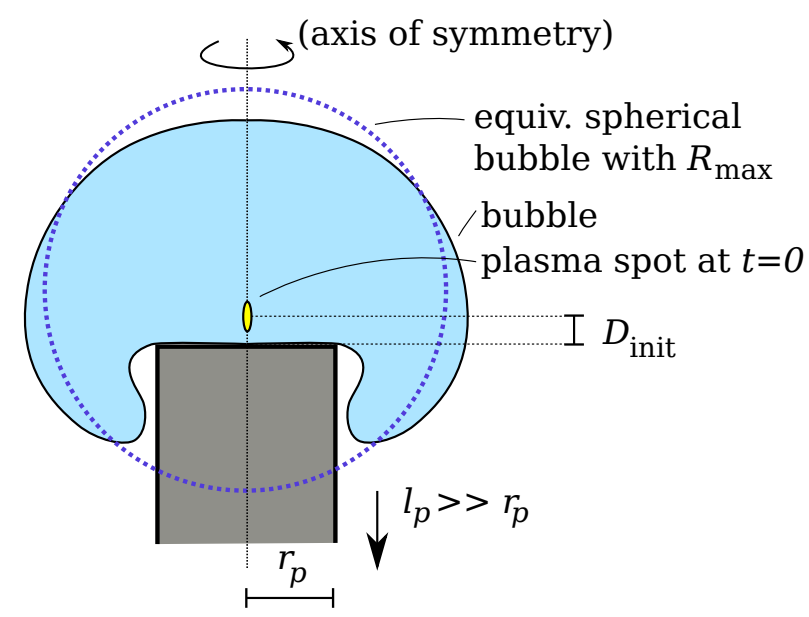

Fig. 7.1: Parameters for classification of bubbles close to a rigid cylinder. The equivalent spherical bubble is understood as the bubble with same energy in unbounded liquid.

The bubble close to a rigid cylinder has interesting dynamics - with strong association to mushroom shapes. Here, the case where the bubble is generated at a point on the axis of symmetry of the cylinder is investigated. Fig. 7.1 shows the parameters of the case. There is one more parameter compared to the case of a bubble close to a planar, solid boundary, which is the radius of the cylinder. $D^{*}$ is now defined the following way:

$$
D^{*}=\frac{D_{\text {init }}}{R_{\text {max,unbound }}}+\frac{R_{\text {max,unbound }}+D_{\text {init }}}{r_{p}} .
$$

This definition i) ensures comparability to the $D^{*}$ of the bubble close to a planar, solid boundary for $r_{p} \rightarrow \infty$; ii) for $r_{p} \rightarrow 0, D^{*}$ tends to infinity giving the unbounded case; iii) it is asymmetric in the three parameters; and iv) it is scale invariant.

In Sec. 7.1 the experimental results, using setup 3 (Sec. 3.3.3), are given for one specific laser energy. At the time of experiment conduction, a lot of effort has been made to film the moment of neck closure. The numerics afterwards revealed, however, that the neck never closes, because it is pierced by a fast jet. Again, its existence was indirectly shown. In Sec. 7.2 the measurements for three different laser energies are shown.

In Sec. 7.3.1, the numerics will be tested for convergence, which is a demanding task, since a lot of small splashing phenomena occur that are likely to alter the solutions very much with altering the grid. In Sec. 7.3, the parameter space is investigated. The fast-jet happens at the axis of symmetry for all bubbles presented. There is a parameter sub-space where the fast jet happens inside a nearly cylindrical bubble. This could be another candidate to investigate the fast-jet phenomenon experimentally. This is interesting for future projects. In most of the cases presented however, the real 3D bubble does not have a smooth cylinder surface to see through. 
Again, the existence of the fast-jet phenomenon was indirectly measured by numerical interpolation and numerical interpretation.

In this chapter, the overlay method is skipped because the bubbles are rather thin than voluminous and their shape is also clearly seen in the experiment.

\subsection{Constant bubble energy}

As an introduction to the interesting dynamics of a bubble on top of a rigid cylinder the sequence in Fig. 7.2 is given, depicting a stacking/ interleaving of 4 measurements from the Photron APXRS view, each recorded at $21000 \mathrm{fps}$ with a resolution of $384 \times 272$ pixels. By interleaving the measurements of the repetitive bubbles, an approximate time resolution of $84 \mathrm{kfps}$ is obtained. The needle was indeed a sewing pin needle, which was ground to flat-top by diamond grinding by the workshop of the institute. The needle top has a diameter of $(545.6 \pm 5.9) \mu \mathrm{m}$.

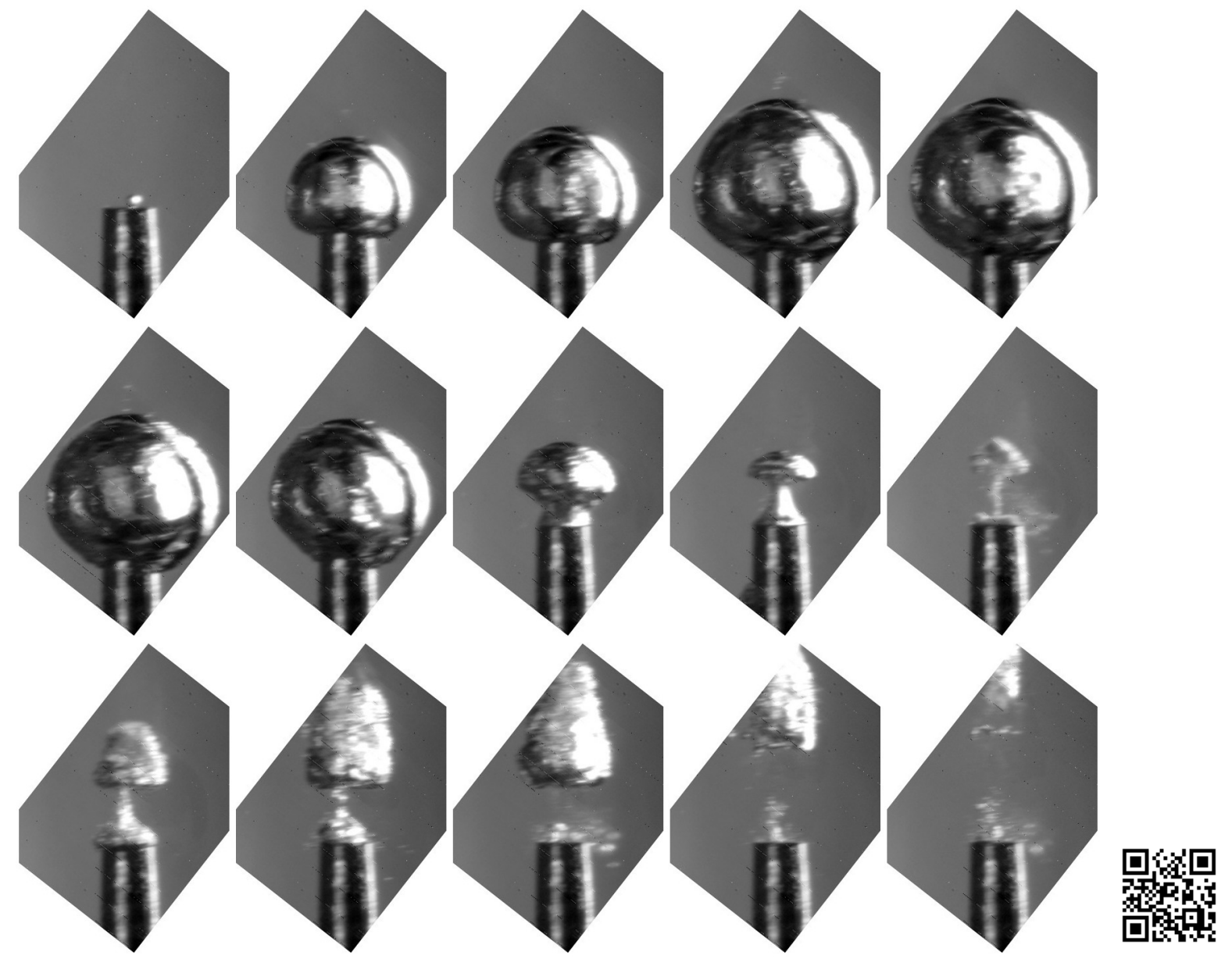

Fig. 7.2: Experimental images of a bubble generated at the center of the top surface of a metal cylinder. Exposure time is $1 \mu \mathrm{s}$. Stacked sequences of 4 recordings with the Photron camera at $21 \mathrm{kfps}$ each. Frame rate for frames 1-7: $\approx 42 \mathrm{kfps}$; for frames 7-12: $\approx 84 \mathrm{kfps}$; for frames $12-15: \approx 42 \mathrm{kfps}$. Raw video of one measurement online via QR code or: https://doi.org/10.25625/3R8KTX

The main aspects of the dynamics are already visible here, e.g. the bubble being put over the cylinder edge during expansion. Before final collapse, a neck is formed, together with the mushroom's head. The bubble collapse turns the mushroom shape into a blurred one, but 
the neck seems to persist. In the rebound the former mushroom head gets very large again and detaches from the needle like a projectile. When viewing the raw video of one of the experiments (link in Fig. 7.2), the shaking of the needle due to the momentum can be observed, too.

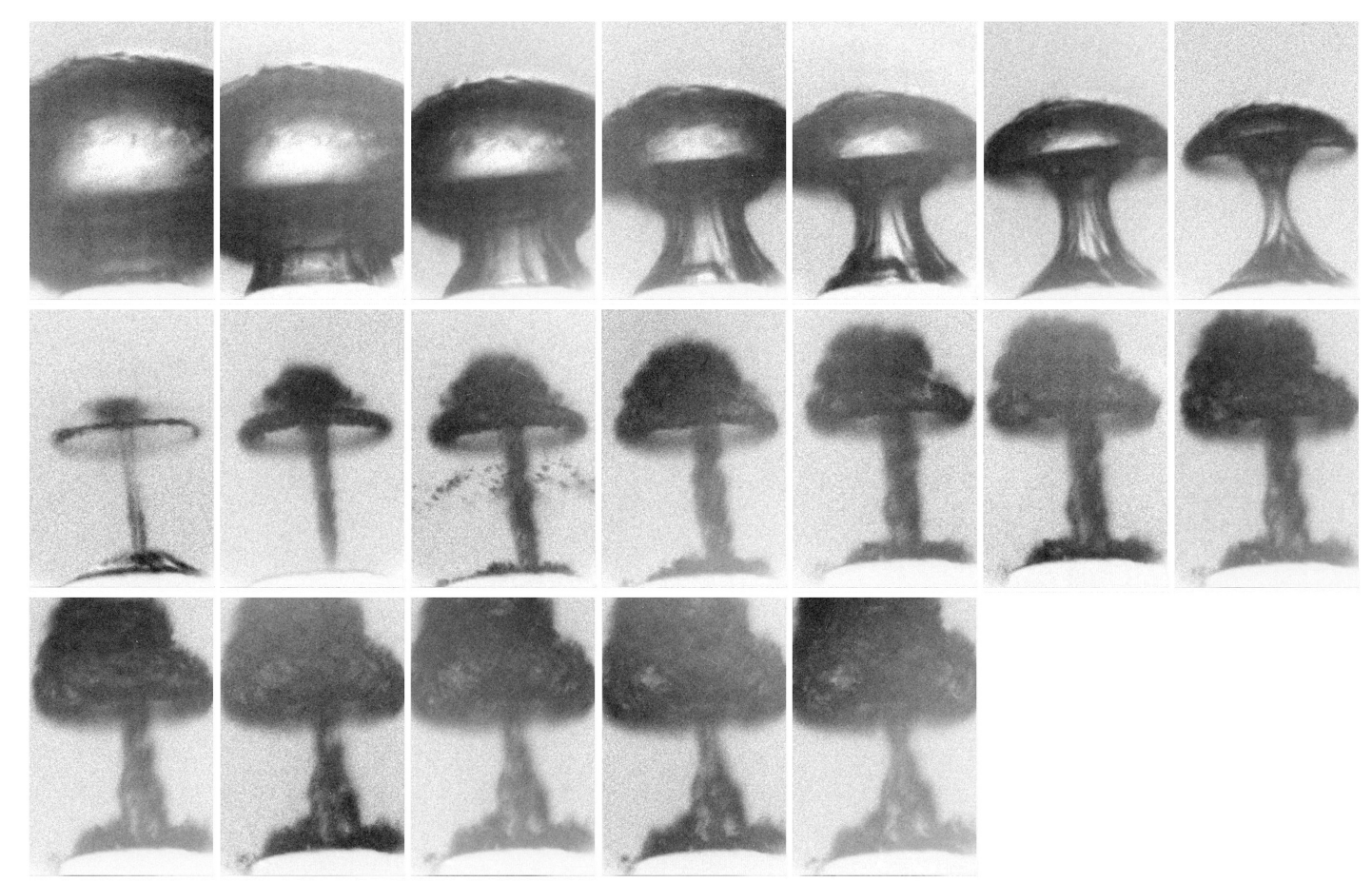

Fig. 7.3: Experimental images of a bubble similar to Fig. 7.2, but with higher frame rate recorded with the IMACON camera. Exposure time is $150 \mathrm{~ns}$. Sequences of 3 recordings are stacked together. Frame rate for frames 1-3: $317 \mathrm{kfps}$ (2150 ns interval time); for frames 4-end: $870 \mathrm{kfps}$ (1150 ns interval time). Background of the images was subtracted.

The corresponding Imacon camera recordings allow for a more detailed insight into the temporal dynamics of the peculiar collapse dynamics (Fig. 7.3) at a frame rate of mainly $870 \mathrm{kfps}$ and $150 \mathrm{~ns}$ exposure time. In the first row, the observer expects a violent neck closure in the middle of the neck of frame 7 , where it is narrowest. The more peculiar is the apparent, perfectly straight cylindrical neck in frame 8 and the mushroom cap thin like a jellyfish. In the early rebound in frame 10, supposedly secondary cavitation leads to tiny bubbles around the neck, ironically reminding of the mushroom annulus in real mushrooms. Afterwards the mushroom turns into a cloudy projectile. What happens in the very collapse moment, where the bubble showed us its miraculous shape? In Fig. 7.4, five frames of 5 prominent measurements are put together which show the moment of collapse. Assuming again repetitivity of the measurements, it seems that the bulk gas "under the roof" of the mushroom shrinks to the top below the roof while the neck becomes more tapered homogeneously on the full length. 

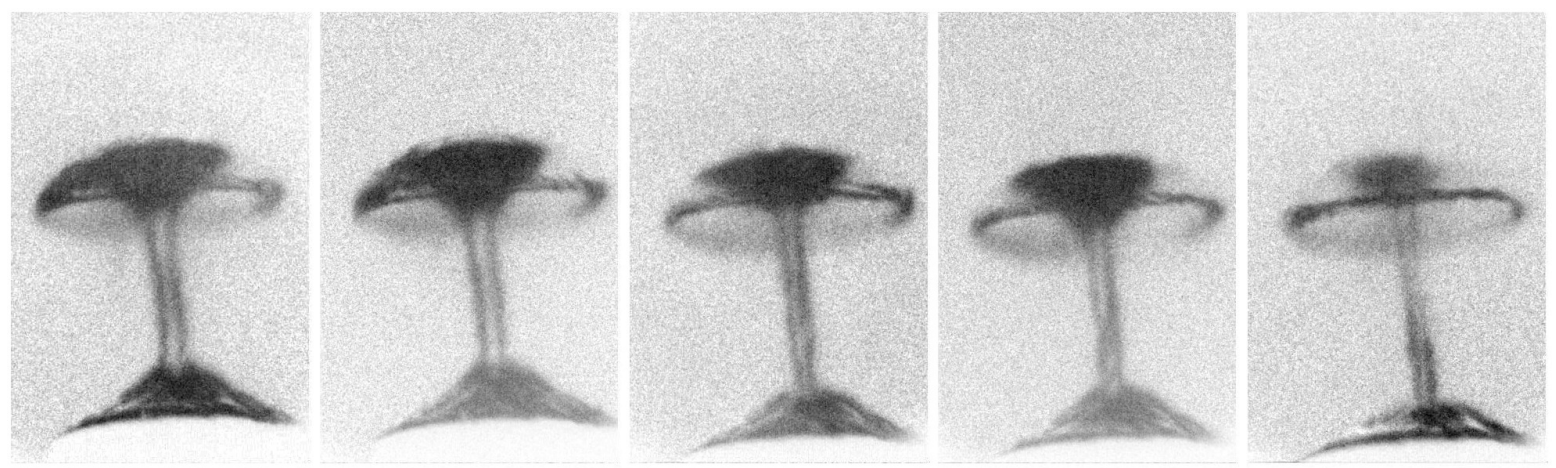

Fig. 7.4: Extracted experimental images - from recordings similar to Fig. 7.3 - where the mushroom shape is thinnest.

Therefore, more insight into the very moment before the first frame of Fig. 7.4 is needed. In Fig. 7.5, the record sequences of the subsequent experiment are stacked to elucidate the dynamics of neck formation. The exposure time has been reduced to $30 \mathrm{~ns}$ and partly $10 \mathrm{Mfps}$ could be used. This is the highest time resolution gained in this thesis, even per measurement, proving that the experiment setup 3 works very well. It is observed that in frame 8 the neck closure is expected to happen but somehow the neck just becomes more translucent. In the further frames, the "bottom stand foot" is formed and its peak seems to "slide down" the neck. The bottom stand foot further decreases its height while the top mushroom collapses first in the upper part, subsequently in the lower part. The bottom stand foot has not reached zero height yet, when the upper mushroom parts are already expanding again cloudly in their rebound phases.

These observations are to be kept in mind over the next section, where different bubble energies are investigated, until the numerics will tell what actually is going on. 


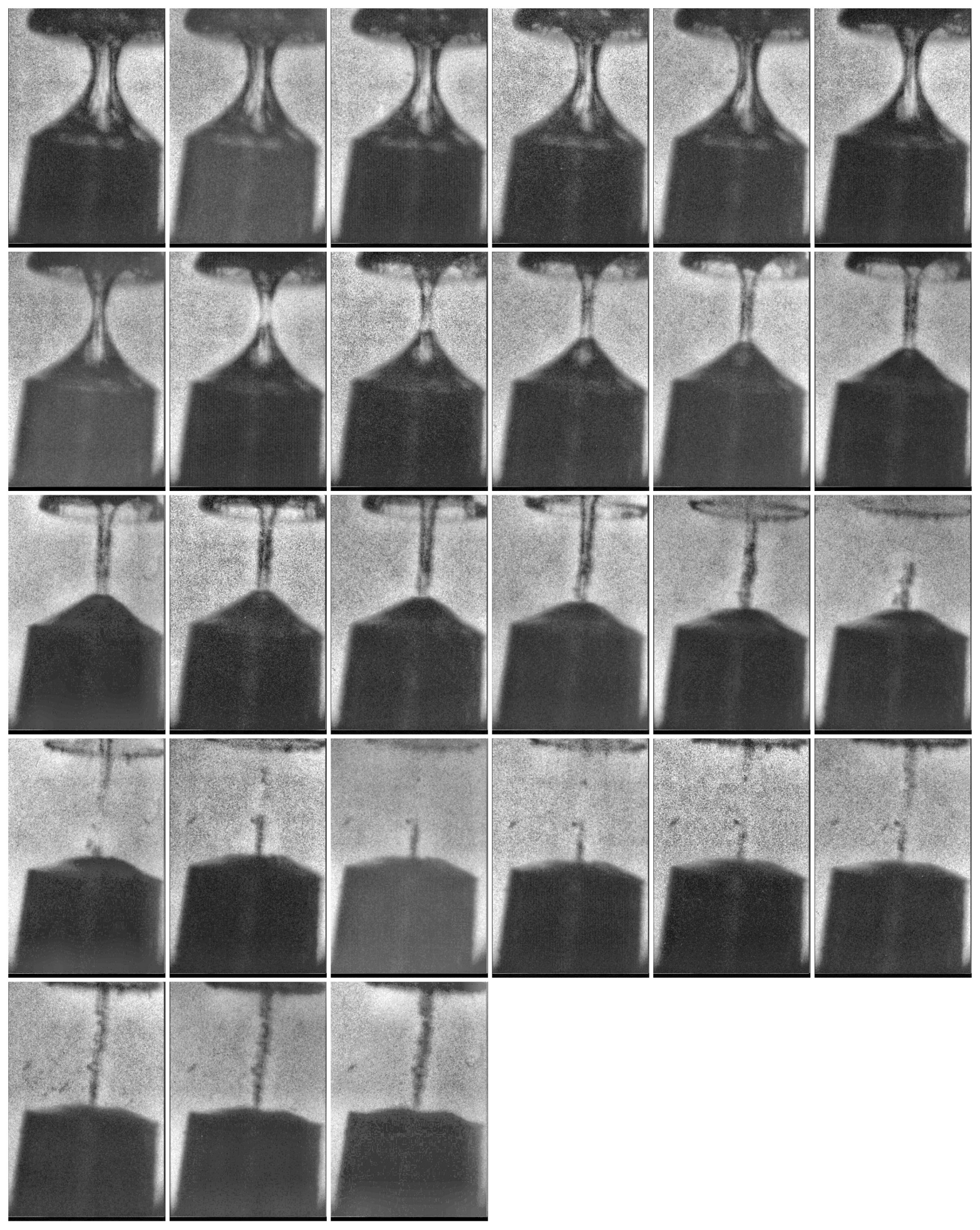

Fig. 7.5: Experimental images of a bubble similar to Figs. 7.2 and 7.3, but with ultra high frame rate. Exposure time is $30 \mathrm{~ns}$. Frame rate and interval times (int) are as follows: Frames 1-13: $10 \mathrm{Mfps}=$ int 100 ns; Frames 14-19: 5.6 Mfps = int $180 \mathrm{~ns}$; Frames 20-27: $10 \mathrm{Mfps}=$ int $100 \mathrm{~ns} ; 5$ recordings stacked together: Frame 1-5, 6-13, $14-17,18-19,20-27$ 


\subsection{Different bubble energies}

In this section, three different laser energies are investigated. It is found that the mushroom analogy is not persistent to a wide range of energies, at least not as clear. For each energy two to three Imacon measurements are complemented with the respective photodiode signal and Photron recording of one of the bubbles.

It seems that this series was recorded with the plasma breakdown location slightly off-axis of the cylinder needle. This can be seen in the Photron views for late time instants. Even with this slight asymmetry, however, the differences in the dynamics are evident to the eye.

Starting with the higher energy than in the previous section, shown in Fig. 7.6, it is seen that the dynamics are more easily affected by asymmetry. This is intuitive in the sense that the degrees of freedom of liquid inflow increase when the cylinder diameter becomes smaller compared to the maximum radius, while at the same time the position of the cylinder axis relatively matters more. The former mushroom cap turns into a mere ring at the top of the bubble and a larger amount of bulk gas is pushed into the upper half of the collapsing congadrum-shaped bubble.

The mushroom shape could be reproduced sufficiently by applying a similar energy as in the previous section (Fig. 7.7). The perspective is more orthogonal to the cylinder this time, but the straight neck is seen again and the bulk gas that is pushed into the top below the mushroom cap. The mushroom annulus is not seen, though, which is supposed to happen around the sixth frame of the bottom measurement, compared to the Fig. 7.3. Therefore, it may be guessed that the annulus formation is sensitive to perfect symmetry.

The bubble with lower energy (Fig. 7.8) than the one in the previous section was recorded with ultra-high speed framerates. The dynamics are extremely fast and the shape reminds of a tree stub. The gas bulk this time is thinnest under the former mushroom cap and the neck remains thick.

The time from bubble generation to collapse is about $195 \mu$ s for the highest energy, $160 \mu$ s for the middle and $100 \mu$ s for the lowest energy. 

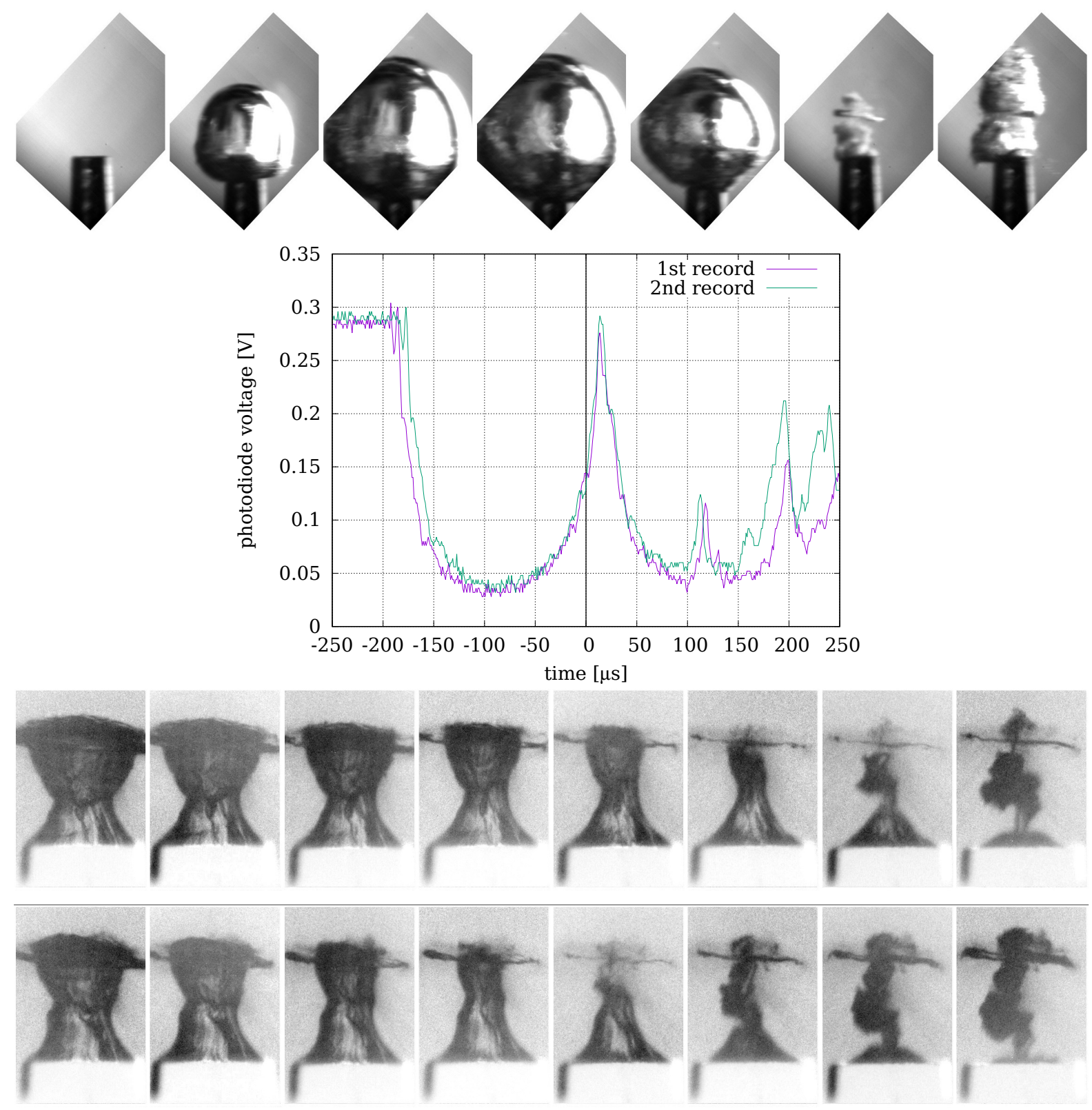

Fig. 7.6: Mushroom bubble with more energy to the ones in Sec. 7.1. The two Imacon measurements (bottom) were recorded with $2.5 \mathrm{Mfps}$ (400 ns interval time) and exposure time $150 \mathrm{~ns}$. The photodiode signal (middle) of the two recordings has time zero at the moment of triggering. Top: corresponding Photron view. 

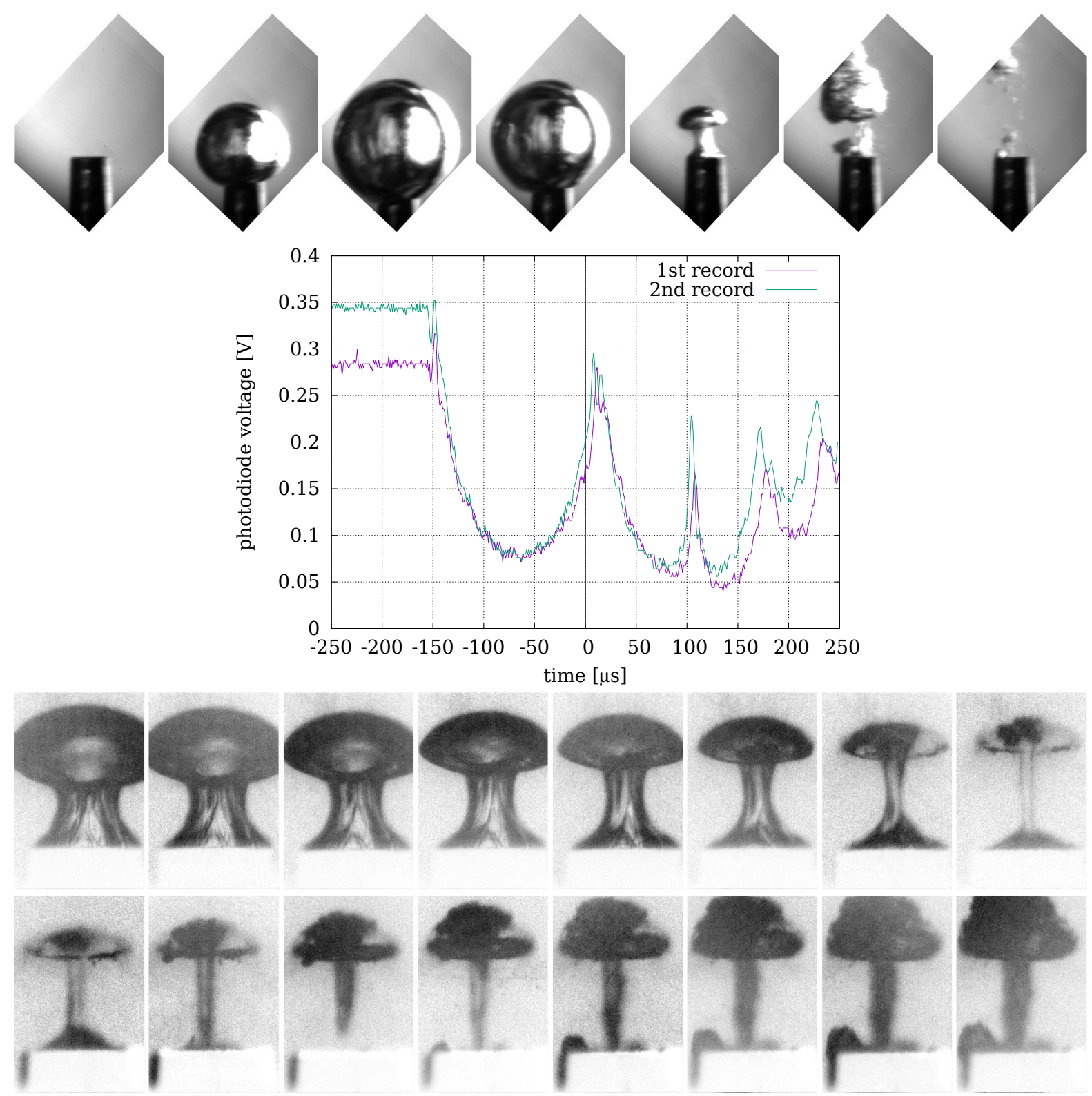

Fig. 7.7: Mushroom bubble with similar energy to the ones in Sec. 7.1. The two Imacon measurements (bottom) were recorded with $650 \mathrm{~ns}$ interval time $(1,538,461.5 \mathrm{Mfps})$ and $150 \mathrm{~ns}$ exposure time. The middle diagram shows the photodiode signal of the two measurements with trigger time at $t=0$ and the top row shows the Photron view of one of the bubbles. 

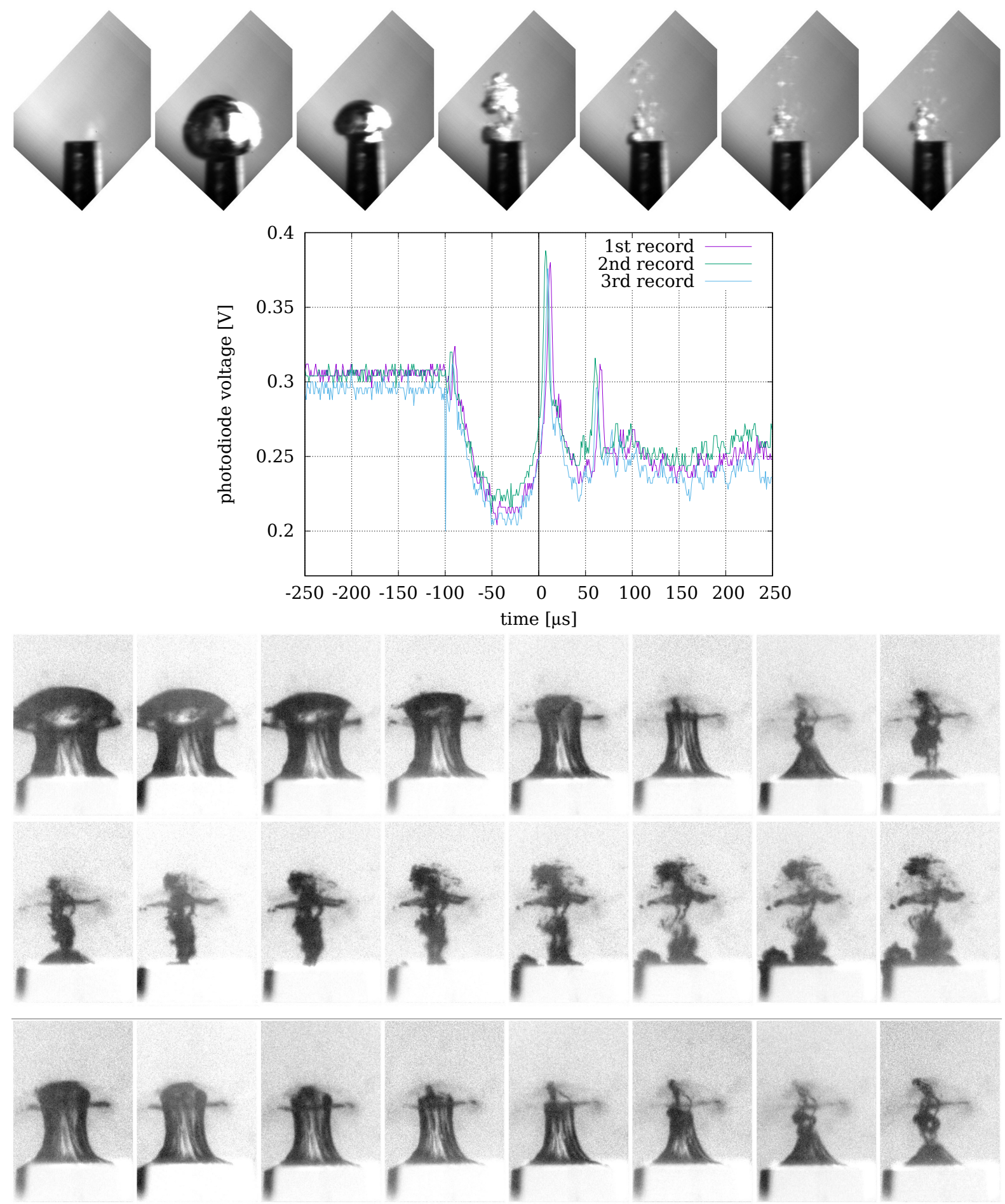

Fig. 7.8: Mushroom bubble with less energy to the ones in Sec. 7.1. The top two Imacon measurements (bottom) above the black line were recorded with $650 \mathrm{~ns}$ interval time $(1,538,461.5 \mathrm{Mfps})$ and $150 \mathrm{~ns}$ exposure time of each frame. The bottom measurement was recorded with $4 \mathrm{Mfps}$ ( $250 \mathrm{~ns}$ interval time) and $150 \mathrm{~ns}$ exposure time. The middle diagram shows the photodiode signals of the measurements with the trigger time at $t=0$. Top row shows the Photron record of one of the bottom row bubbles. 


\subsection{Simulations}

The CFD simulations are performed in axial symmetry and for distinct cases in full 3D. For all simulations, the surface tension was set to zero because instabilities arose in the symmetry point at the top of the cylinder and the axis of symmetry (see Sec. B.2). The reason is most probably an unresolved incompatibility with snappyHexMesh. For three cases $\left(D_{\text {init }}=250 \mu \mathrm{m}\right)$ the results are compared to the ones obtained with $\sigma=0.0725$, showing that until the nonphysical part the results match. For the investigations in axial symmetry the Mesh 3.1.2.a is used with an additional snappyHexMesh step to cut out the cylinder. The radius $r_{p}$ of the cylinder was set to $200 \mu \mathrm{m}$, a little bit smaller than in the experiment. Therefore, the simulations and the experiment cannot be compared 1:1, unless the same $D^{*}$ is applied, assuming same dynamics for same $D^{*}$ for this type of boundary. The mesh parameters are

$$
\delta x_{\min }=1 \mu \mathrm{m}, \mathrm{Xi}=80 \mu \mathrm{m}, \mathrm{Xii}=1.2 \sqrt{2} \mathrm{Xi}, \mathrm{X}=1.04 \mathrm{~mm}, g_{f}=5.25, \mathrm{XF}=80 R_{\max } .
$$

The bubble parameters studied are:

$$
D_{\text {init }} \in[30 \mu \mathrm{m}, 90 \mu \mathrm{m}, 150 \mu \mathrm{m}, 250 \mu \mathrm{m}], R_{n, 1} \in[184.1 \mu \mathrm{m}, 201.5 \mu \mathrm{m}, 240 \mu \mathrm{m}] .
$$

The maximum radii in unbounded liquid are found to be $498.66 \mu \mathrm{m} 554.84 \mu \mathrm{m}$ and $679.16 \mu \mathrm{m}$ for the respective $R_{n, 1}$ by Eq. 3.5. Therefore, the investigated spectrum is $D^{*} \in[2.70,5.01]$.

This is visualized in Fig. 7.9 where the 3D function Eq. 7.1 is plotted for fixed $r_{p}$ and the above data points are put in, too. Some of the $D^{*}$ values are quite similar. Therefore, a statement about the applicability of Eq. 7.1 will be done.

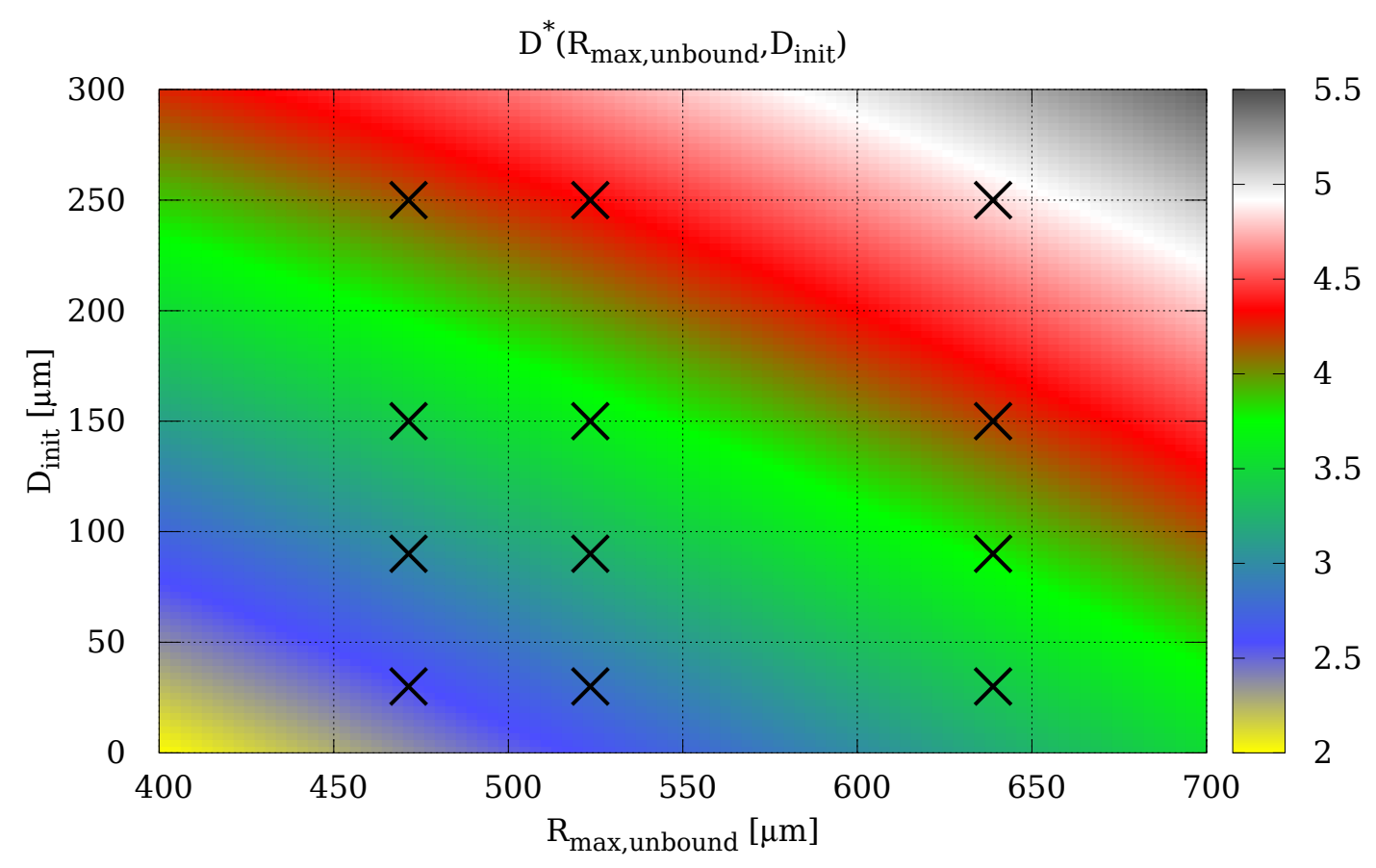

Fig. 7.9: Color plot of Eq. 7.1 is plotted for fixed $r_{p}=200 \mu \mathrm{m}$ and the data points for the investigated cases. 


\subsubsection{Convergence test numerics}

A small convergence test of the bubble shape is given in Fig. 7.10 before the results are presented. The bubble parameters are:

$$
\begin{array}{r}
R_{\text {init }}=20 \mu \mathrm{m}, R_{n, 1}=201.57 \mu \mathrm{m}, R_{n, 2}=64 \mu \mathrm{m}, \\
R_{n} \text { reduction in interval }[60 \mu \mathrm{s}, 75 \mu \mathrm{s}], D_{\text {init }}=30 \mu \mathrm{m}
\end{array}
$$

It is observed that the shape is captured well at a resolution of $1 \mu \mathrm{m}$, assuming the resolution of $0.65 \mu \mathrm{m}$ as the converged solution. Figure 7.11 shows the calculation times in hours on a logarithmic scale. The $D^{*}$ investigations were performed with $1 \mu \mathrm{m}$ resolution which is why the set of data points is highest there.

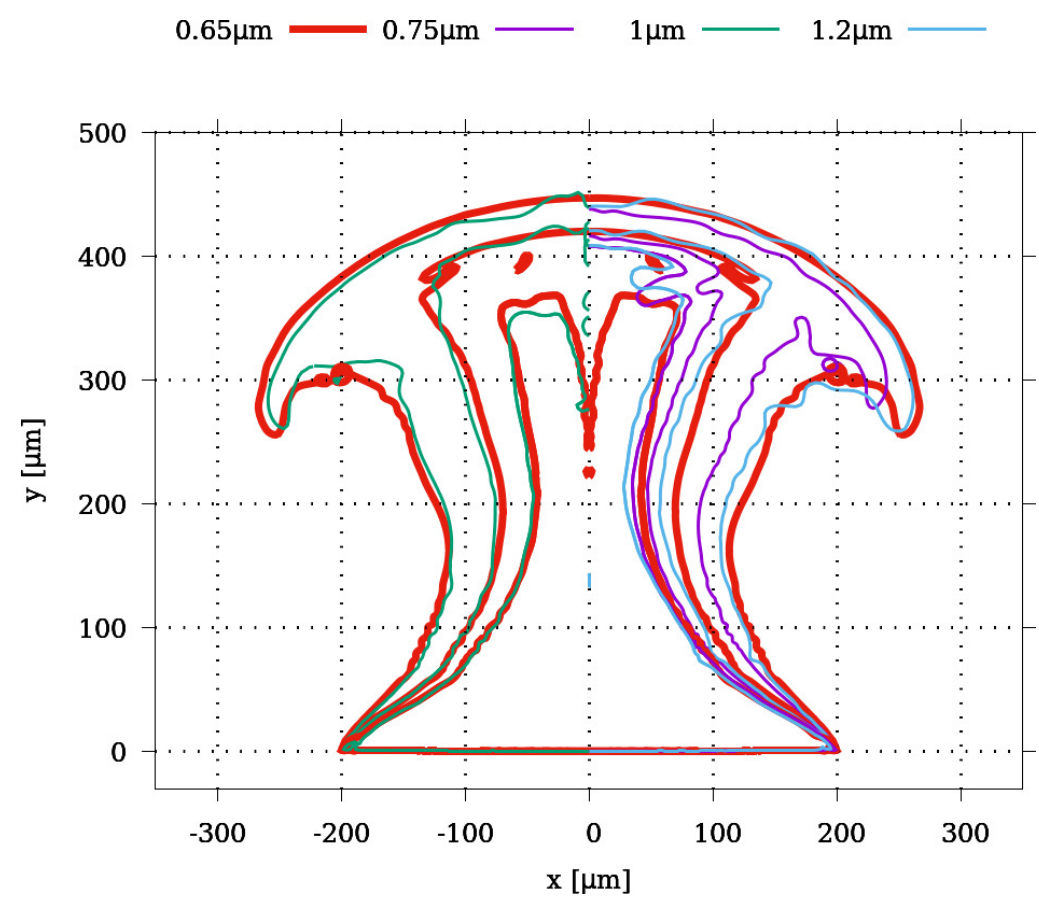

Fig. 7.10: Contours of the bubble shape of axisymmetric calculations of a bubble on a the rigid cylinder for 4 resolutions and 3 time instants. Times for each contour taken at the time of same equivalent radius.

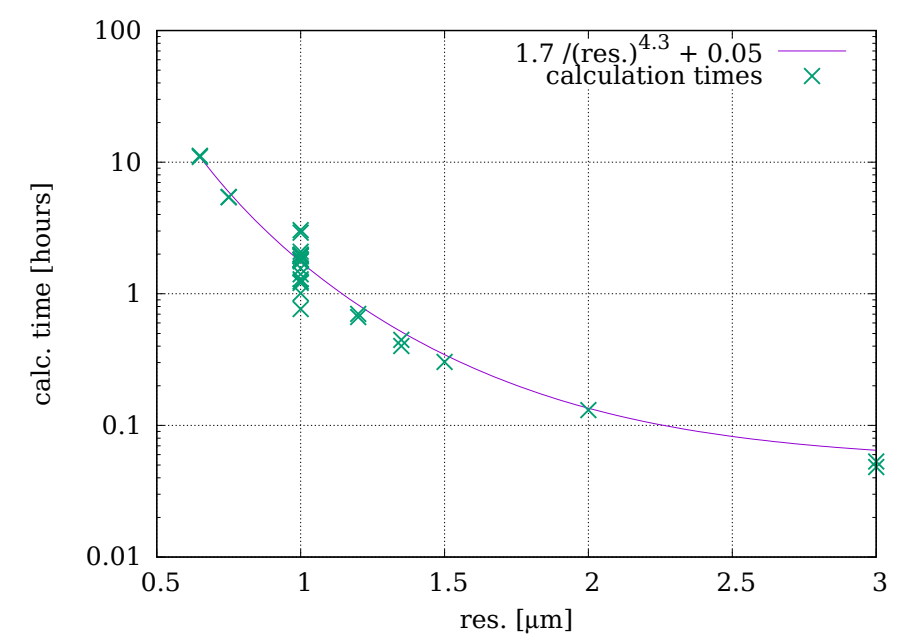

Fig. 7.11: Calculation times for the bubbles of this chapter for $109 \mu \mathrm{s}$. 


\subsubsection{General dynamics}

Here, the general dynamics process is explained in Fig. 7.12 and 7.13. It was found to be similar for all $D^{*}$ cases investigated, as will become clear in the next section. Therefore, the questions that arose from the experimental data, are answered here, too.

Fig. 7.12: During expansion, when the bubble interface crosses the cylinder edges, it swirls around them, ejecting liquid droplets (in axial symmetry torus ring drops) into the bubble body $(5.37 \mu \mathrm{s}, 27.193 \mu \mathrm{s})$. Once passed, these droplets hit again the outer bubble wall, inducing random surface waves there $(27.193 \mu \mathrm{s})$. Due to the boundary layer around the cylinder, the bubble never touches the solid, but "swims" on the boundary layer. This is evident from the numerics close to any solid boundary, but cannot be easily observed in the experiment. When the bubble starts collapsing, the outer waist is lifted almost parallel to the cylinder $(51.857 \mu$ s and $70.116 \mu \mathrm{s}$ ). This flow produces a) the neck and b) the extreme curvatures at the mushroom cap rim. The phenomenon of flow focusing comes into play again here, generating strongest acceleration where curvatures are highest.

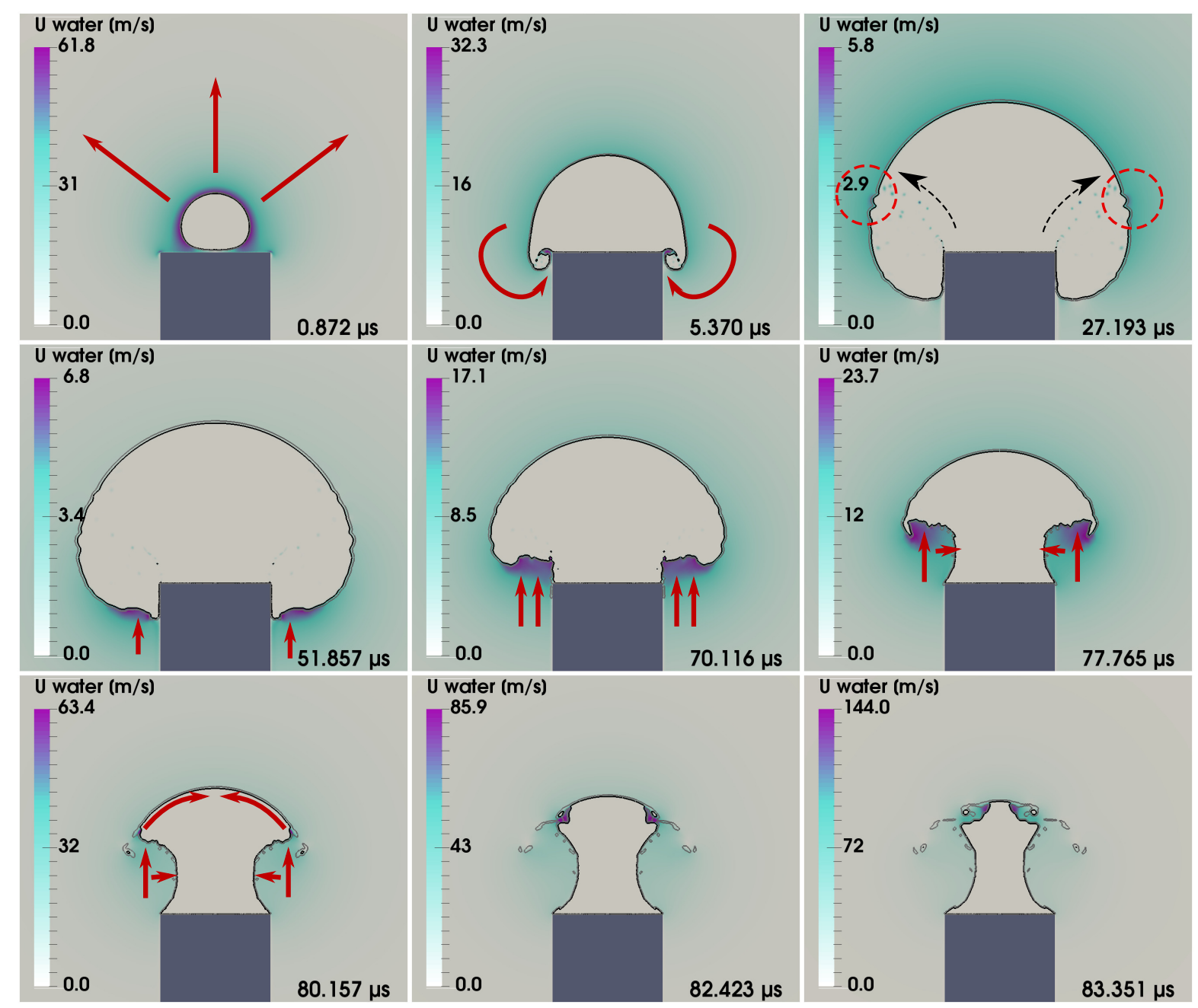

Fig. 7.12: General dynamics of the mushroom bubble, part 1, plotted with the liquid velocity field. Explanation see text. A few flow directions and patterns are indicated by red arrows. 
So an annular jet is formed that runs along the inside of the mushroom cap, leaving trails of dim remnant gas that form a thin umbrella $(80.157 \mu \mathrm{s}, 82.423 \mu \mathrm{s})$. Numerically, this results into a tearing of the interface, leaving areas where $0.9<\alpha_{l}<1$. In the end, this umbrella jet impacts in the zenith of the umbrella, producing - again - a fast jet. This fast jet is actually the reason why the neck closure won't happen. This can be seen in Fig. 7.13: The fast jet here easily reaches values over $1000 \mathrm{~m} / \mathrm{s}$. The liquid inflow from the top now makes all sideways inflows negligible, changing the dynamics to an axial jet. The neck is tapered from inside rather than from outside flows. The experimentalist observes the aforementioned "bottom stand foot" here, because the outside bubble surface has too many wrinkles to see the jet inside. The minimum volume happens from top-down, thus the top gas fragments are already in the rebound phase when the lower ones collapse and emit shockwaves. Therefore, the upper bubbles are "kicked" and squeezed upwards.

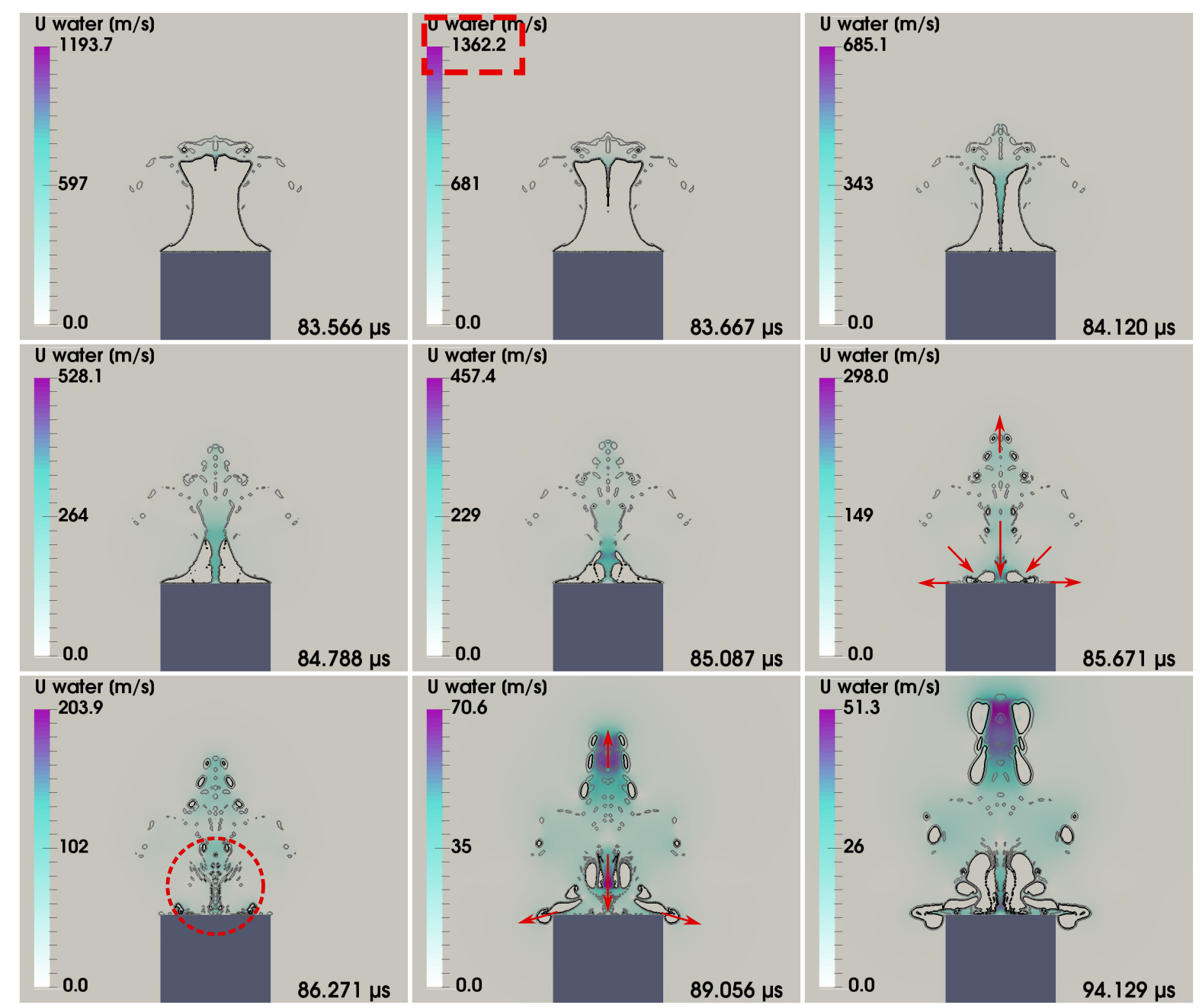

Fig. 7.13: General dynamics of the mushroom bubble, part 2, plotted with the liquid-velocity field. Explanation see text.

\subsubsection{Several $D^{*}$}

All prerequisites are fullfilled to study the $D^{*}$-dependence. In the following Figs. 7.14 and Figs. 7.15 the bubbles are plotted with their liquid velocity field, sorted according to the $D^{*}$ - 
Eq. 7.1, for four prominent times each: 1 - lifting the hips above the cylinder edge, 2 - annular jet impact, 3 - fast-jet impact on the top of the cylinder and 4 - about bubble minimum volume.

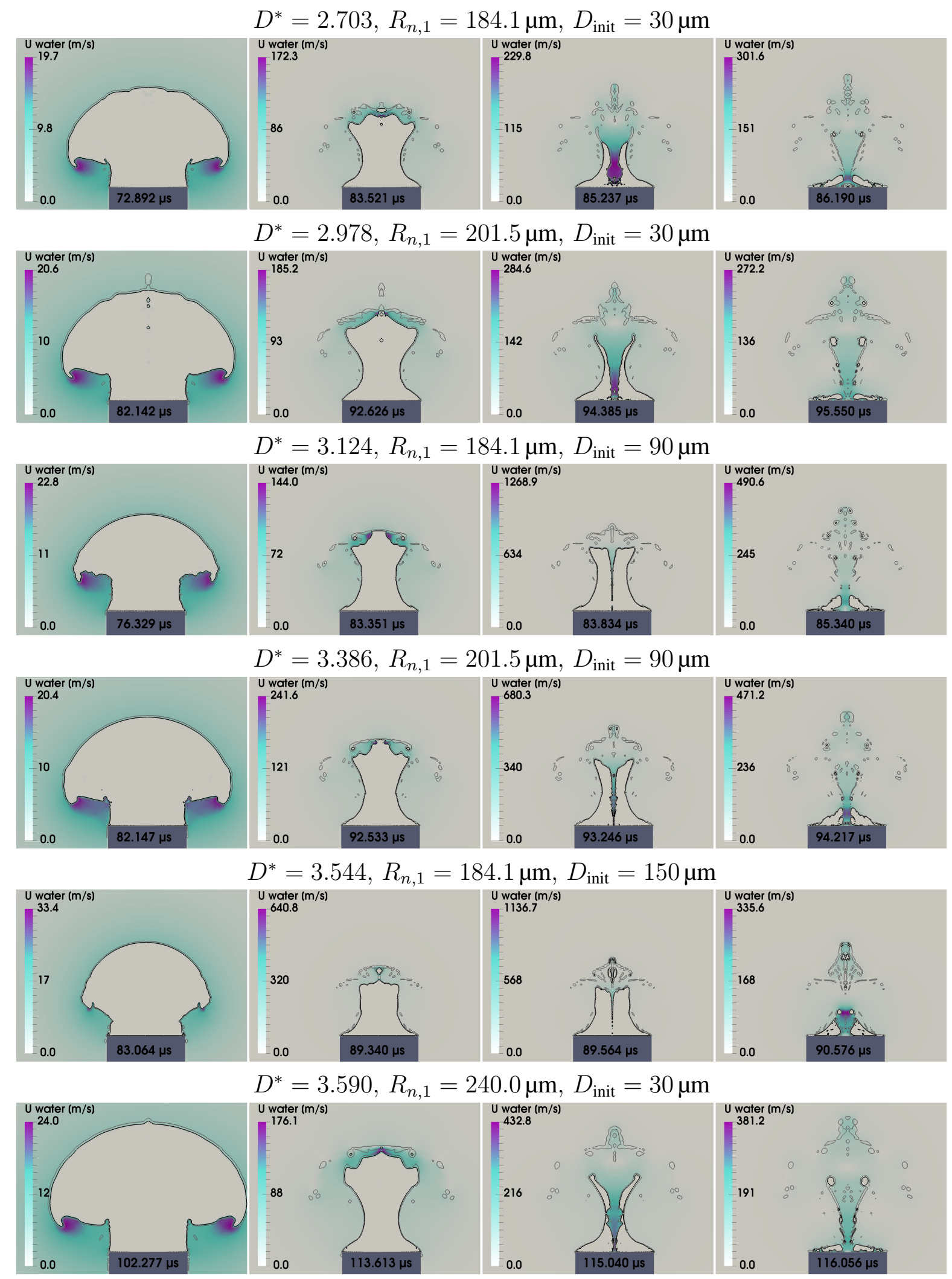

Fig. 7.14: Mushroom bubble $D^{*}$-dependence part 1. Sorting according to Eq. 7.1. 

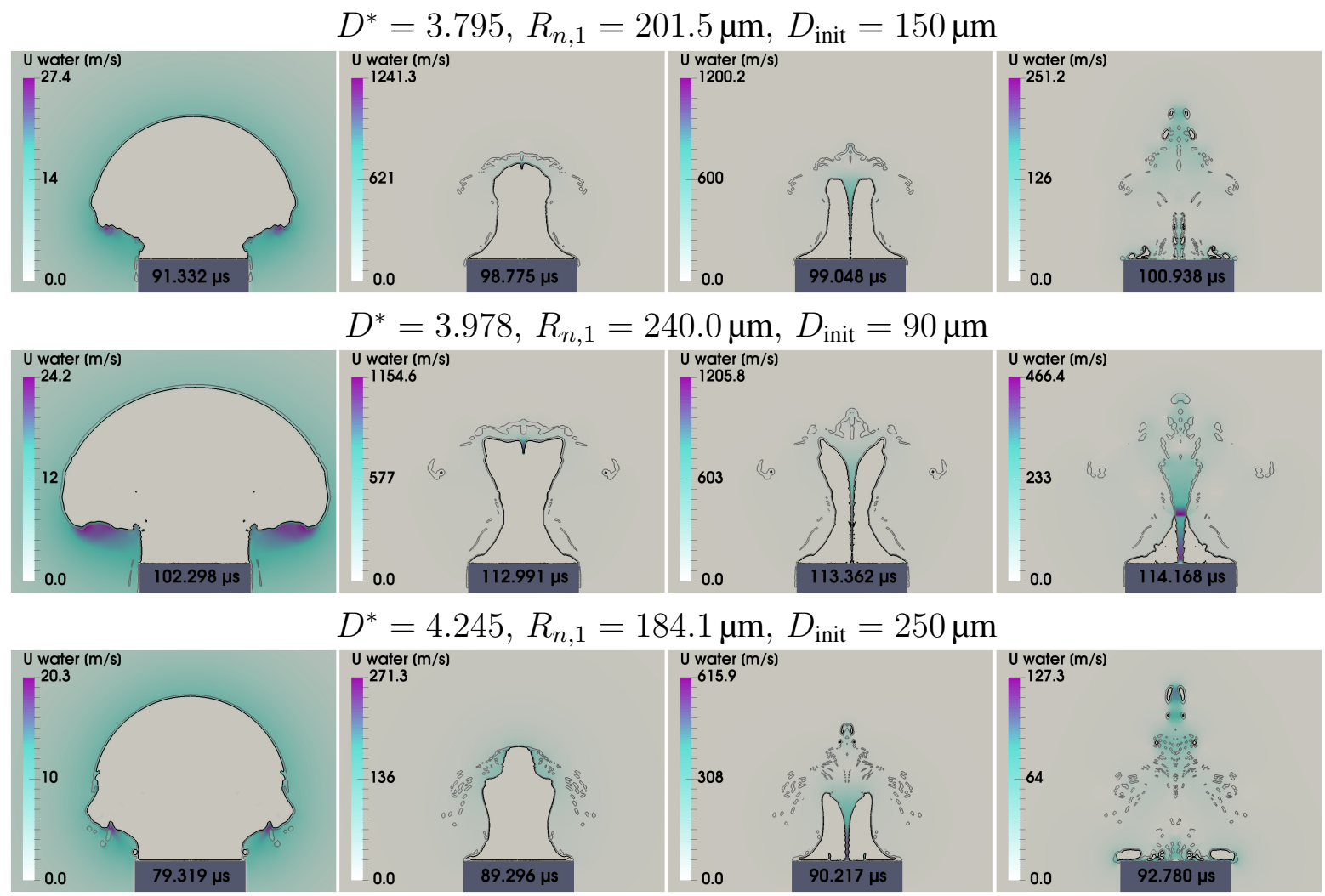

$D^{*}=4.367, R_{n, 1}=240.0 \mu \mathrm{m}, D_{\text {init }}=150 \mu \mathrm{m}$

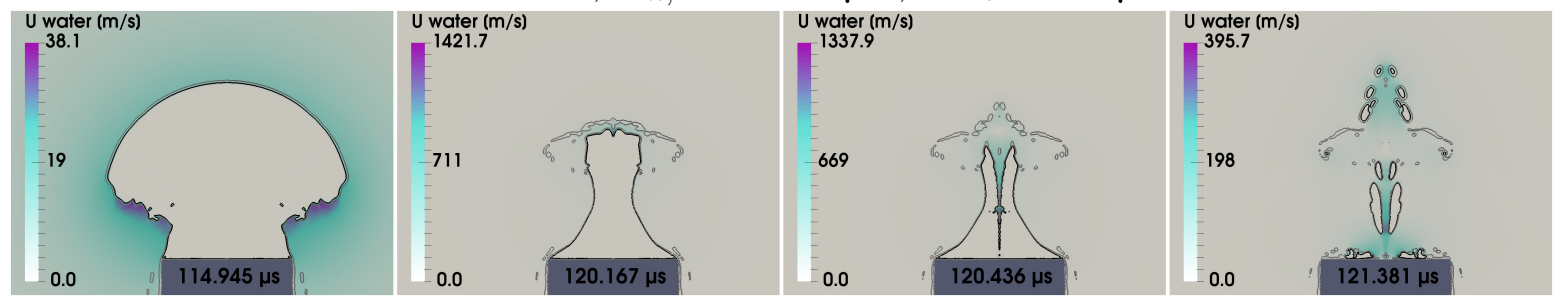

$D^{*}=4.475, R_{n, 1}=201.5 \mu \mathrm{m}, D_{\text {init }}=250 \mu \mathrm{m}$

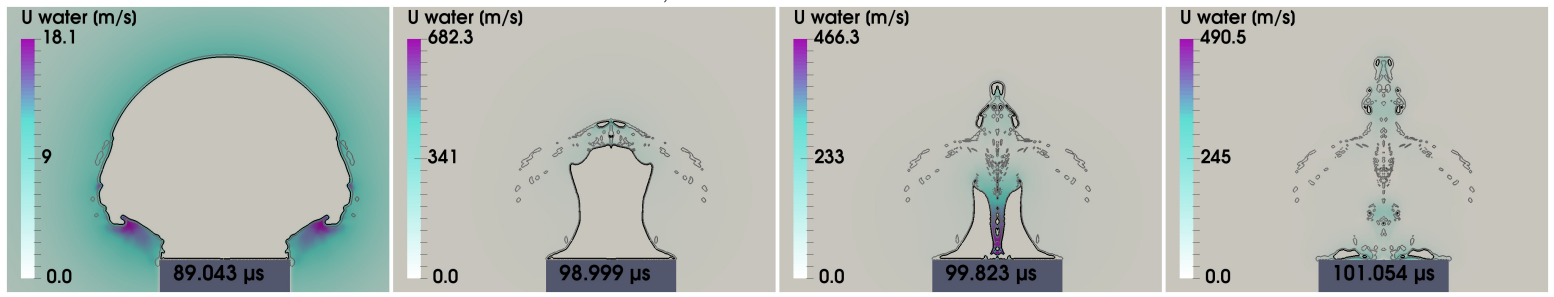

$D^{*}=5.014, R_{n, 1}=240.0 \mu \mathrm{m}, D_{\text {init }}=250 \mu \mathrm{m}$

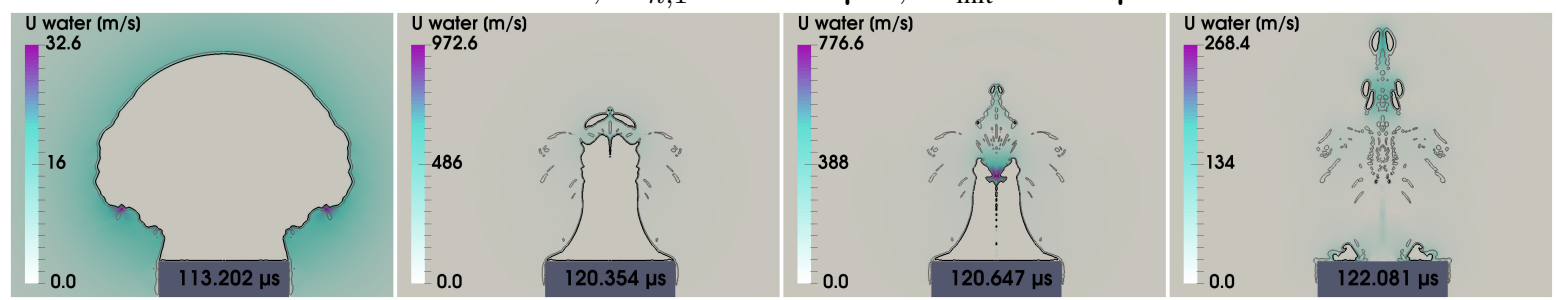

Fig. 7.15: Mushroom bubble $D^{*}$-dependence part 2. Sorting according to Eq. 7.1.

To conclude, judging the bubble shape at annular jet impact (each second frame) the sorting according to Eq. 7.1 works reasonably well, except for the bubbles at $D^{*}=3.544$ and $D^{*}=$ 3.978 , whose positions in the sorting should be exchanged. 


\subsubsection{Two distinct simulations in full 3D}

The following images show a montage of two simulations in full 3D, revealing that the axisymmetric calculations capture the main dynamics very well. The contour surfaces of $\alpha_{l}=0.9$ (light gray, transparent) and $\alpha_{l}=0.5$ (green, transparent) are plotted. The higher value for $\alpha_{l}$ accounts for the dim gas remnants that are sub-resolution. The mesh used violated many of the best practice meshing guidelines of this thesis, but the intention was to show the qualitative perfect agreement with i) the results in axial symmetry and ii) the experimental observations.

\section{$7.331 \mu \mathrm{s}$}
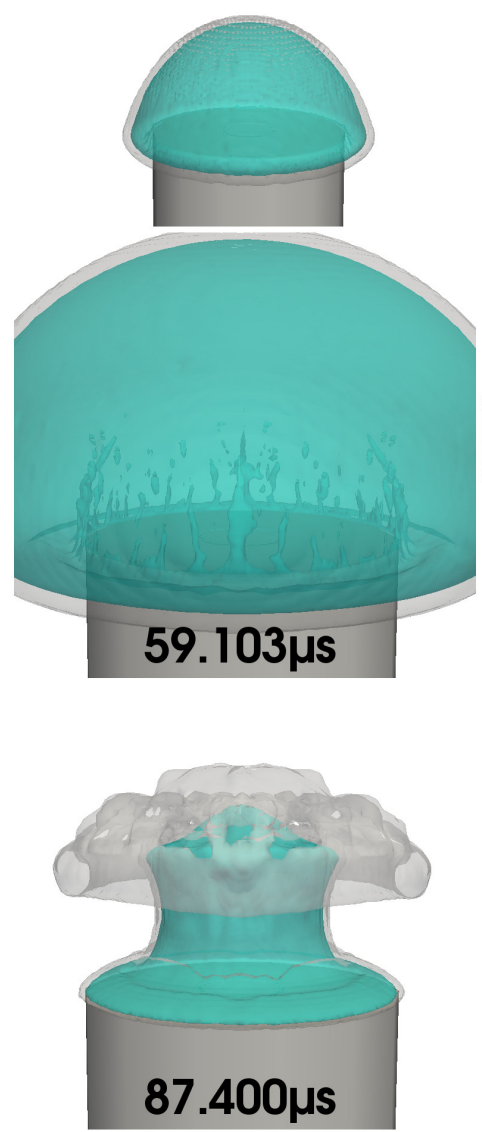

$90.590 \mu \mathrm{s}$

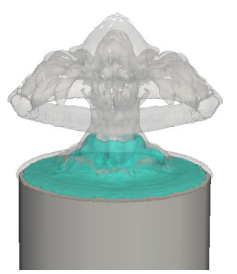

$32.103 \mu \mathrm{s}$
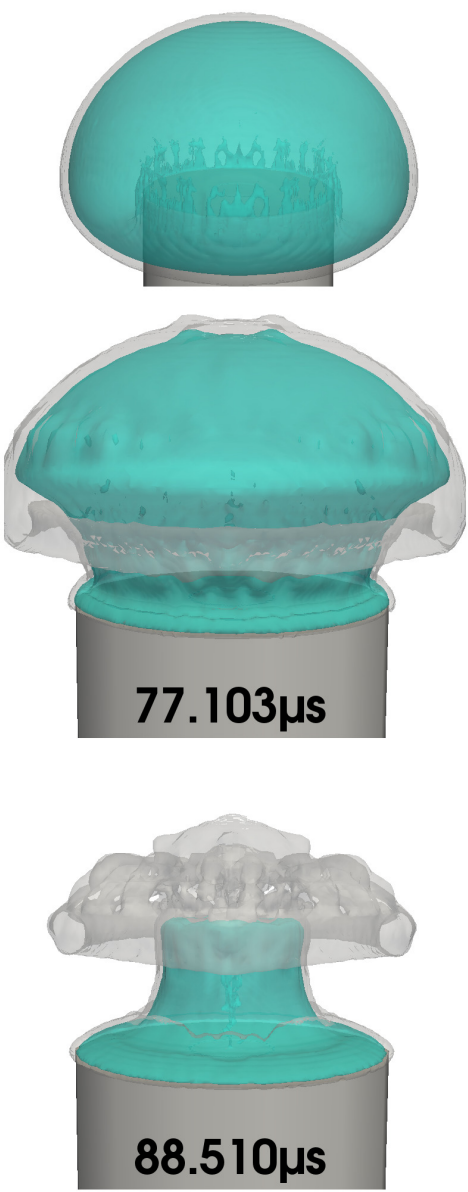

$99.698 \mu \mathrm{s}$

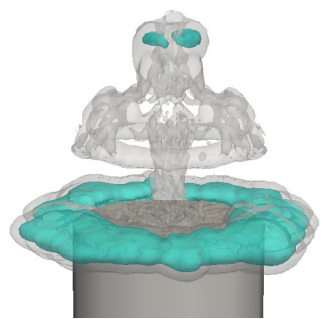

$50.103 \mu \mathrm{s}$
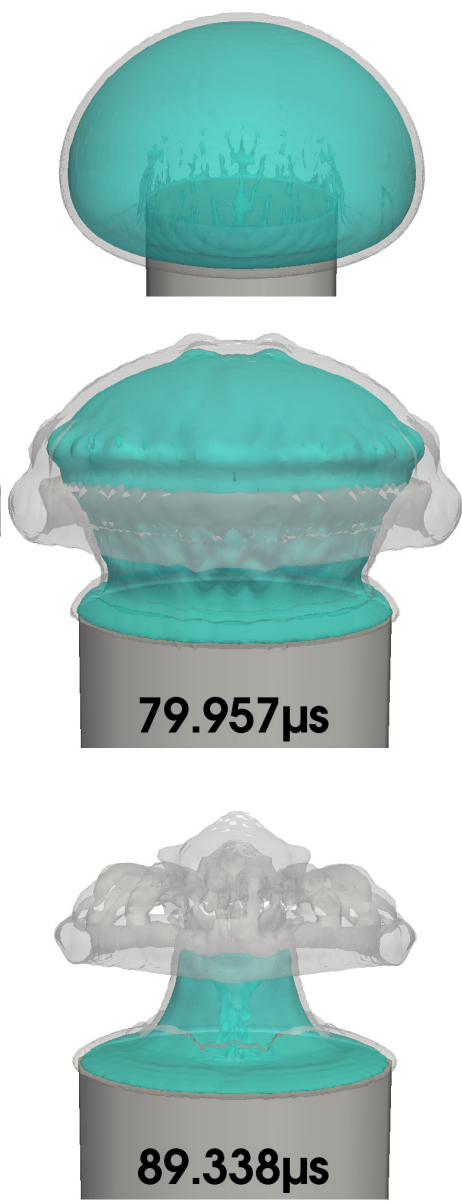

$108.387 \mu s$

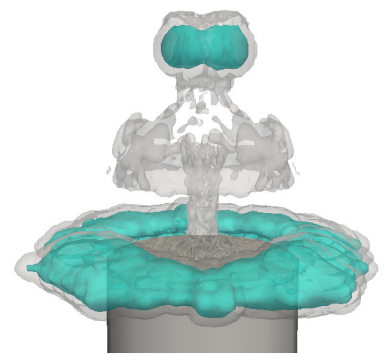

Fig. 7.16: Full 3D simulation for $D^{*}=1.88, r_{p}=320 \mu \mathrm{m}, R_{n, 1}=201.57 \mu \mathrm{m}, D_{\text {init }}=30 \mu \mathrm{m}$, showing the "tree stub" dynamics. 


\section{$5.394 \mu s$}
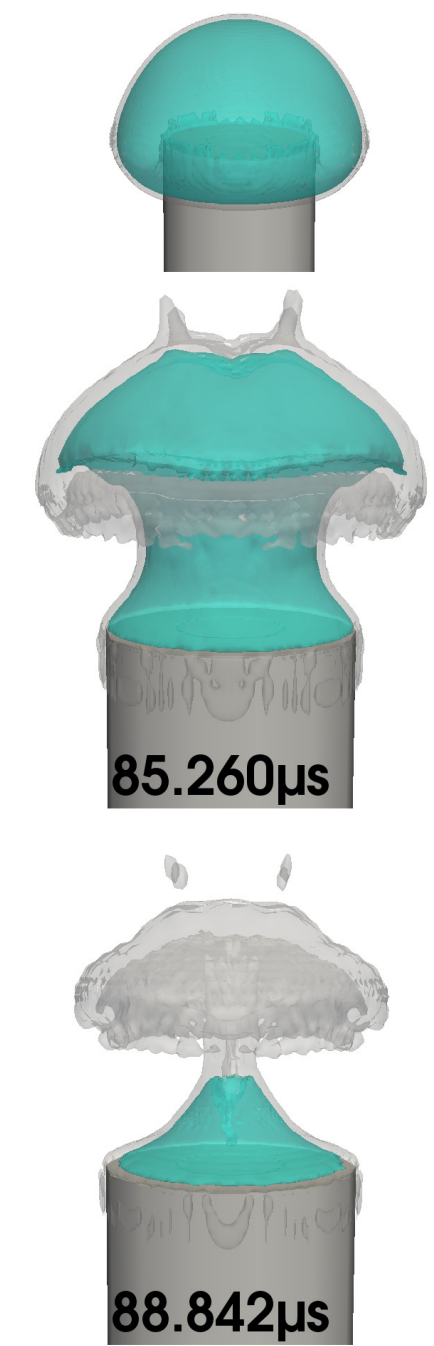

$103.553 \mu \mathrm{s}$

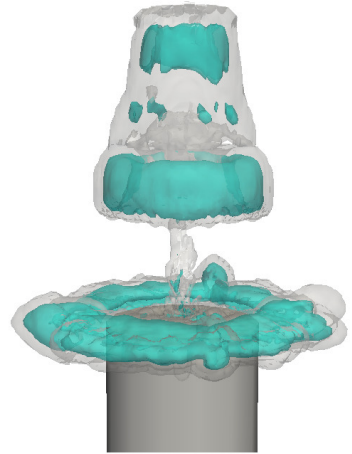

$30.170 \mu s$
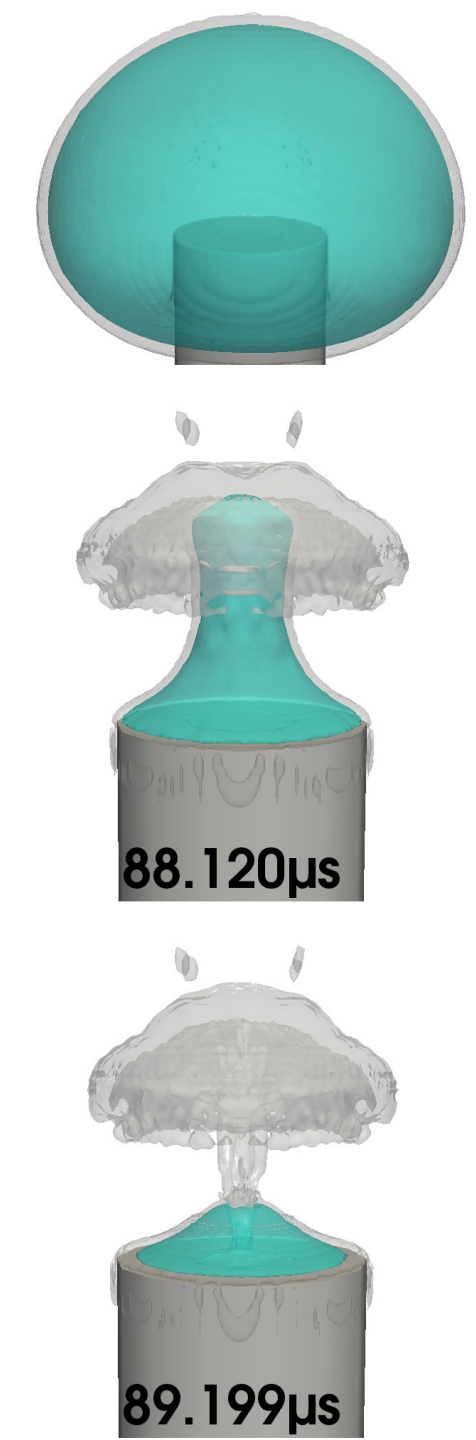

$112.613 \mu \mathrm{s}$

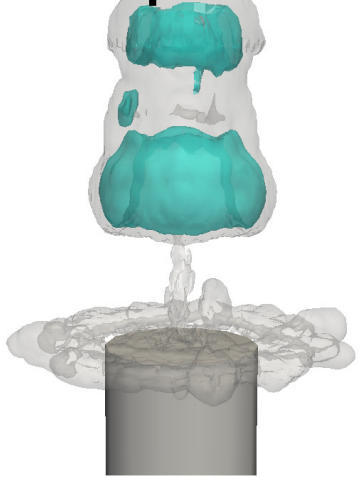

75. $170 \mu \mathrm{s}$
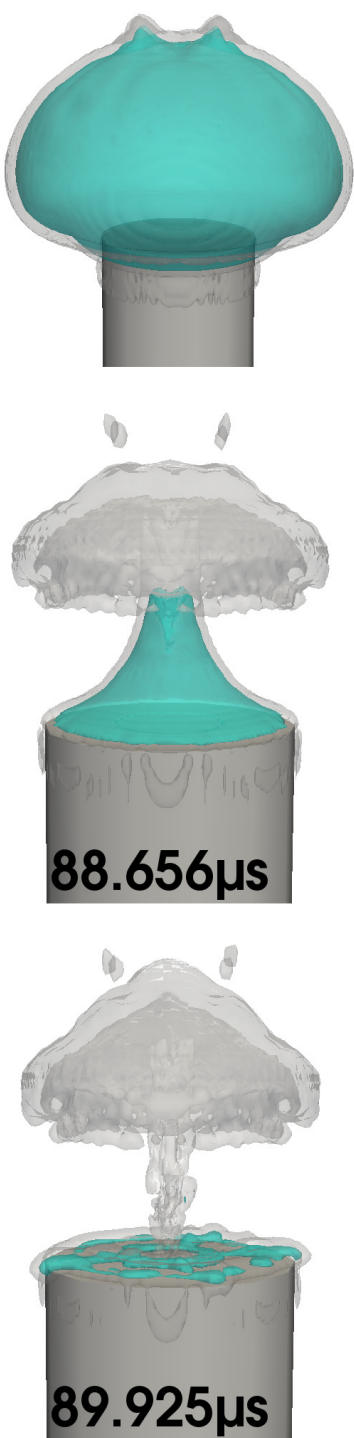

$138.542 \mu s$

Fig. 7.17: Full 3D simulation for $D^{*}=2.979, r_{p}=200 \mu \mathrm{m}, R_{n, 1}=201.57 \mu \mathrm{m}, D_{\text {init }}=30 \mu \mathrm{m}$, showing the "mushroom dynamics" including the projectile. 


\section{Conclusion}

The main body of this thesis deals with the existence of the so called fast jet that develops when a single, laser generated cavitation bubble expands and collapses close to a flat, solid boundary at normalised distances of $D^{*} \in[0,0.2]$. One reason for this focus is that even after 100 years of research on cavitation erosion, the precise mechanism of damage of hardest materials by cavitation bubbles still is not fully clear. Philipp and Lauterborn (1998) measured the erosion pit volume of a single cavitation bubble on a soft material of $16 \mathrm{MPa}$ yield strength (aluminum) in a range of the normalised distance $\gamma_{d} \in[0.3,3]$ to the solid boundary. In this thesis it is shown in Sec. 6.3 that exactly in the range $\gamma_{d} \approx D^{*} \in[0,0.3]$ the main pressure peak takes place in the symmetry point at the solid boundary below the bubble.

In order to arrive at this conclusion, both the numerical two-phase compressible solver for the Navier-Stokes equation had to be developed to an elaborate extent and the experimental methods had to be designed for high precision records of the bubble collapse instant. The solver has been described in Sec. 2.4.1. It covers non-linear, isentropic compressibility of bubble and liquid at sub-, trans- and supersonic flow speeds, as well as gas phase splitting. It is based on the compressibleInterFoam solver of OpenFOAM. The experiments have been described in Sec. 3.3. A complex trigger circuit was established to repeatedly film the collapse moment of a single, laser generated bubble at Megaframes per second.

One extra step that was further necessary, was to compare numerical and experimental results. The ray-tracing method, shipped with the $3 \mathrm{D}$-software blender, made it possible to transform the numerical results into images that look very similar to the ones obtained from the high speed photography experiments. This way the interpretation of the experimental results could reach beyond optical limits. The validation of the method has been given in Sec. 3.2.1. A more detailed and quantitative validation has found its way into the open-access publication of Koch et al. (2020).

In order to give trust into the results of the code, experimental results from Reuter et al. (2017a) for the long-term fluid motions around a bubble have been reproduced numerically with success in Ch. 4. The authors measured vortices that either travel alongside or away from the solid boundary, depending on the normalised distance.

By coincidence, the investigation of bubbles above a solid cylinder (shown in Ch. 7) supports the opinion that a fast jet should exist in general. The bubbles were generated directly on the flat top of a long, solid cylinder with diameter comparable or less than the maximum radius of the bubble. Interestingly, the shape of the bubbles that forms during collapse reminds of a mushroom. Moreover, the stem/ neck of the mushroom narrows rapidly before minimum bubble volume, but does not impact onto itself. A clear stem remains. This could be explained by the simulations: A fast jet piercing the stem from top to bottom suddenly changes the flow characteristics.

To conclude, it can be said that the fast jet most probably exists. The arguments are:

- The bubbles of the ray-traced simulation and the experiment look very alike. The time interval, where the fast jet can be filmed is about $100 \mathrm{~ns}$, thus it is likely to be missed. 
- The neck (stem) of the mushroom shaped bubbles above a solid cylinder does not selfimpact. The numerics reveal that instead the neck is penetrated by a fast jet and subsequently disintegrated.

A first evaluation of the pressure peak of nearly $4 \mathrm{GPa}$ that a bubble produces onto a flat, solid boundary in the range $D^{*} \in[0,0.2]$ reveals that it is actually not produced by the fast jet (see Sec. 6.3). It is rather produced by focusing of the torus shockwaves of the second collapse.

This observation shows once more that the intriguing phenomena of the bubble collapse at objects still have to be explored further in the future, and that the present work represents one more step on the way towards a complete understanding of cavitation erosion. 


\section{Bibliography}

Aitken, Frédéric and Jean-Numa Foulc (2019). From Deep Sea to Laboratory 3 - From Tait's Work on the Compressibility of Seawater to Equations-of-State for Liquids. Wiley.

Asaithambi, Niveditha, Poonam Singha, Madhuresh Dwivedi, and Sushil K. Singh (2019). Hydrodynamic cavitation and its application in food and beverage industry: A review. Journal of Food Process Engineering 42.5, e13144. DOI: 10.1111 / jfpe. 13144.

$\mathrm{Au}$, Whitlow W. L. and Kiara Banks (1998). The acoustics of the snapping shrimp Synalpheus parneomeris in Kaneohe Bay. JASA 103.1, pp. 41-47. DoI: $10.1121 / 1.423234$.

Bahl, Simon and Markus Schönekeß (2020). LED flash circuit. Elektronic workshop Drittes Physikalisches Institut, Georg-August Universität Göttingen (see appendix).

Benjamin, T. B. and A. T. Ellis (1966). The Collapse of Cavitation Bubbles and the Pressures thereby Produced against Solid Boundaries. Philosophical Transactions of the Royal Society of London, Series A: Mathematical and Physical Sciences 260, pp. 221-240.

Blake, John R., David M. Leppinen, and Qianxi Wang (2015). Cavitation and bubble dynamics: the Kelvin impulse and its applications. Interface Focus 5, p. 20150017.

Blender Foundation and Community (v. 2.82). Cycles - Blender Manual. English. https://docs.blender.org/manual/en/latest/render/cycles/index.html.

Brackbill, J. U., D. B. Kothe, and C. Zemach (1992). A Continuum Method for Modeling Surface Tension. Journal of Computational Physics 100, pp. 335-354.

Brennen, C. E. (1995). Cavitation and Bubble Dynamics. Oxford University Press.

Brennen, C. E. (2015). Cavitation in medicine. Interface Focus 5, p. 20150022. DOI: 10 . 1098 /rsfs.2015.0022.

Carnelli, Davide, Ayat Karimi, and Jean-Pierre Frac (2011). Application of spherical nanoindentation to determine the pressure of cavitation impacts from pitting tests. Journal of Materials Research 27.1, pp.91-99. DOI: 10.1557 / jmr.2011.259.

Chahine, Georges L. (2014). Modeling of cavitation dynamics and interaction with material. Advanced Experimental and Numerical Techniques for Cavitation Erosion Prediction. Ed. by K. H. Kim, G. L. Chahine, J.-P. Franc, and A. Karimi. Fluid Mechanics and Its Applications. Springer Netherlands. Chap. 6, pp. $123-161$.

Chahine, Georges L. and Chao-Tsung Hsiao (2015). Modelling cavitation erosion using fluid material interaction simulations. Interface Focus 5, p. 20150016. DOI: 10 . 1098 / rsfs . 2015.0016.

Chaplin, Martin F. (2019). Structure and Properties of Water in its Various States. Encyclopedia of Water. American Cancer Society, pp. 1-19. DOI: $10.1002 / 9781119300762$. wst s 0002 .

Cheng, Lijing, John Abraham, Zeke Hausfather, and Kevin Trenberth (2019). How fast are the oceans warming? American Association for the Advancement of Science 363.6423, $128 \mathrm{f}$.

Cole, Robert H. (1948). Underwater Explosions. Princeton University Press.

Cornolti, Luca, Tommaso Lucchini, Gianluca Montenegro, and Gianluca D'Errico (2013). A comprehensive Lagrangian flame-kernel model to predict ignition in SI engines. International Journal of Computer Mathematics 91. DOI: 10.1080/00207160.2013.829213. 
European Committee for Standardization (2020). NS-EN ISO-6892-1:2019 Metallic materials Tensile testing, Part 1: Method of test at room temperature. English. Norm.

Franks, Felix (1972). Water - a comprehensive treatise - Vol.1. New York: Plenum Press.

Fujikawa, Shigeo and Teruaki Akamatsu (1980). Effects of the non-equilibrium condensation of vapour on the pressure wave produced by the collapse of a bubble in a liquid. Journal of Fluid Mechanics 97, pp. 481-512.

Geisler, Reinhard (2004). Untersuchungen zur laserinduzierten Kavitation mit Nanosekundenund Femtosekundenlasern (Investigations of laser induced cavitation applying nanosecond and femtosecond lasers). German. PhD-thesis. Universität Göttingen.

Gogate, Parag R. and Abhijeet M. Kabadi (2009). A review of applications of cavitation in biochemical engineering/biotechnology. Biochemical Engineering Journal 44.1, pp. 60-72. DOI: $10.1016 / j . b e j .2008 .10 .006$.

Gschaider, Bernhard, Håkan Nilsson, Henrik Rusche, Hrvoje Jasak, Martin Beaudoin, and Vanja Skuric (2017). Open Source CFD Toolbox. URL: https://sourceforge.net/projects/foam-extend/.

Han, Bing, Karsten Köhler, Kerstin Jungnickel, Robert Mettin, Werner Lauterborn, and Alfred Vogel (2015). Dynamics of laser-induced bubble pairs. Journal of Fluid Mechanics 771, pp. 706-742. DOI: $10.1017 / \mathrm{jfm} .2015 .183$.

Harvie, D.J.E., M.R. Davidson, and M. Rudman (2006). An analysis of parasitic current generation in Volume of Fluid simulations. Applied Mathematical Modelling 30.10, pp. 1056 -1066. DOI: $10.1016 / j$.apm.2005.08.015.

Hsiao, Chao-Tsung, A. Jayaprakash, A. Kapahi, J.-K. Choi, and Georges L. Chahine (2014). Modelling of material pitting from cavitation bubble collapse. Journal of Fluid Mechanics 755, pp. 142-175. DOI: 10.1017 / jfm.2014.394.

Infinity-USA (2016). Model K2 DistaMax Long-Distance Microscope System. https://www.infinityusa.com/wp-content/uploads/2020/04/Model-K2-DistaMax-OEM-Handbook-041320.pdf.

Ishiyama, T., S. Fujikawa, T. Kurz, and W. Lauterborn (2013). Nonequilibrium kinetic boundary condition at the vapor-liquid interface of argon. Physical Review E - Statistical, Nonlinear, and Soft Matter Physics 88.4, p. 042406. DOI: 10.1103/PhysRevE. 88.042406.

Johnsen, Eric and Tim Colonius (2008). Shock-induced collapse of a gas bubble in shockwave lithotripsy. Journal of the Acoustical Society of America 124.

Johnsen, Eric and Tim Colonius (2009). Numerical simulations of non-spherical bubble collapse. Journal of Fluid Mechanics 629, pp. 231 -262.

Key to Metals AG (2020). Total Materia, The worlds's most comprehensive materials database. URL: https://totalmateria.com.

Kobel, Philippe, Danail Obreschkow, Aurèle De Bosset, Nicolas Dorsaz, and Mohamed Farhat (2009). Techniques for generating centimetric drops in microgravity and application to cavitation studies. Experiments in Fluids 47.1, pp. 39-48. DOI: 10 . 1007 / s00348-009$0610-0$.

Koch, Max (2014). Numerical modelling of cavitation bubbles with the finite volume method. English. Master thesis. Georg-August Universität Göttingen, Drittes Physikalisches Institut, C. D. Labor für Kavitation und Mikro-Erosion. DOI: 10.25625 / JVC JVW.

Koch, Max (2020). Github page: python-tools.

URL: https://github.com/ma-tri-x/simple_raytracer.

Koch, Max, Christiane Lechner, Fabian Reuter, Karsten Köhler, Robert Mettin, and Werner Lauterborn (2016). Numerical modeling of laser generated cavitation bubbles with the finite volume and volume of fluid method, using OpenFOAM. Computers \& Fluids 126, pp. 71-90. DOI: $10.1016 / j . c o m p f l u i d .2015 .11 .008$. 
Koch, Max, Juan Manuel Rosselló, Christiane Lechner, Werner Lauterborn, Julian Eisener, and Robert Mettin (2020). Theory-assisted optical ray tracing to extract cavitation-bubble shapes from experiment. Experiments in Fluids. DOI: 10.1007/s00348-020-03075-6.

Kupferinstitut (2010). Kupfer-Aluminium-Legierungen.

Köhler, Karsten (2010). Numerische Untersuchungen zum optischen Durchbruch von Femtosekunden-Laserpulsen in Wasser (Numerical investigations on the optical breakdown of femtosecond laser pulses in water). English. PhD thesis. Drittes Physikalisches Institut, GeorgAugust Universität Göttingen.

Lauer, E., X.Y. Hu, S. Hickel, and N.A. Adams (2012). Numerical modelling and investigation of symmetric and asymmetric cavitation bubble dynamics. Computers \& Fluids 69, pp. 1-19.

Lauterborn, W. (1974). Kavitation durch Laserlicht (Laser-Induced Cavitation). Acustica 31, pp. 51-78.

Lauterborn, W. and H. Bolle (1975). Experimental investigations of cavitation-bubble collapse in the neighbourhood of a solid boundary. Journal of Fluid Mechanics 72, pp. 391-399.

Lauterborn, W. and T. Kurz (2010). Physics of bubble oscillations. Reports on Progress in Physics 73.10, 106501.

Lauterborn, Werner (1980). Cavitation and Inhomogeneities in Underwater Acoustics. Springer New York.

Lauterborn, Werner, Christiane Lechner, Max Koch, and Robert Mettin (2018). Bubble models and real bubbles: Rayleigh and energy-deposit cases in a Tait-compressible liquid. IMA Journal of Applied Mathematics 83, pp. 556-589. DOI: 10.1093 / imamat/hxy 015.

Lechner, Christiane, Max Koch, Fabian Reuter, Karsten Köhler, Robert Mettin, and Werner Lauterborn (2015). Numerical Modeling of Laser-Induced Cavitation Bubbles with a Finite Volume Method. PAMM 15.1, pp. 515-516. DOI: 10.1002 / pamm. 201510248.

Lechner, Christiane, Max Koch, Werner Lauterborn, and Robert Mettin (2017). Pressure and tension waves from bubble collapse near a solid boundary: A numerical approach. Journal of the Acoustical Society of America 142, pp. 3649-3659.

Lechner, Christiane, Max Koch, Werner Lauterborn, and Robert Mettin (2018). Details of the collapse of a cavitation bubble next to a flat, rigid wall. Proceedings of the 10th International Symposium on Cavitation (CAV2018). Ed. by Joseph Katz. New York: ASME. DOI: 10 . 1115/1.861851_ch55.

Lechner, Christiane, Werner Lauterborn, Max Koch, and Robert Mettin (2019). Fast, thin jets from bubbles expanding and collapsing in extreme vicinity to a solid boundary: A numerical study. Phys. Rev. Fluids 4 (2), p. 021601. DOI: 10 .1103/Phy sRevFluids . 4.021601.

Lechner, Christiane, Werner Lauterborn, Max Koch, and Robert Mettin (2020). Jet formation from bubbles near a solid boundary in a compressible liquid: Numerical study of distance dependence. Phys. Rev. Fluids 5 (9), p. 093604. DOI: 10.1103/PhysRevFluids. 5. 093604.

Leighton, T. G. (1994). The Acoustic Bubble. Academic Press.

Li, Yuan-Hui (1967). Equations of State of Water and Sea Water. Journal of Geophysical Research 72.10, pp. 2665-2678.

Lindau, Olgert (2001). Untersuchungen zur lasererzeugten Kavitation (Investigations on laser generated cavitation). German. PhD-thesis. Universität Göttingen.

Lindau, Olgert and Werner Lauterborn (2003). Cinematographic observation of the collapse and rebound of a laser-produced cavitation bubble near a wall. Journal of Fluid Mechanics 479, pp. 327-348. 
Luthman, Elizabeth, Niccolo Cymbalist, Daniel Lang, Graham Candler, and Paul Dimotakis (2019). Simulating schlieren and shadowgraph images from LES data. Experiments in Fluids 60.134, pp. 1432-1114. DOI: 10.1007/s00348-019-2774-6.

Löfstedt, Ritva, Bradley P. Barber, and Seth J. Putterman (1993). Toward a hydrodynamic theory of sonoluminescence. Physics of Fluids A 5.11, pp. 2911-2928.

Minuth-Hadi, Florian Jorg (2017). Einfluss der Beanspruchungsgeschwindigkeit auf das Werkstoffverhalten von Baustahl. German. PhD-thesis. Technische Universität Braunschweig.

Müller, S., P. Helluy, and J. Ballmann (2010). Numerical simulation of a single bubble by compressible two-phase fluids. International Journal for Numerical Methods in Fluids 62, pp. 591-631.

Nobach, Holger (2012). Optische Messtechnik. ISBN: 978-3-86468-206-3. Edition Winterwork.

Noblin, X., N. O. Rojas, J. Westbrook, C. Llorens, M. Argentina, and J. Dumais (2012). The fern sporangium: a unique catapult. American Association for the Advancement of Science 335.6074, pp.1322. DOI: 10.1126/science.1215985.

Ochiai, Naoya, Yuka Iga, Motohiko Nohmi, and Toshiaki Ikohagi (2011). Numerical Analysis of Nonspherical Bubble Collapse Behavior and Induced Impulsive Pressure during First and Second Collapses near the Wall Boundary. Journal of Fluid Science and Technology 6.6, pp. 860-874.

Ohl, Claus-Dieter (1998). Zur Dynamik und Lumineszenz von Kavitationsblasen (On the dynamics and luminenscence of cavitation bubbles). German. PhD-thesis. Universität Göttingen.

Ohl, Claus-Dieter and Emmanuel Ory (2000). Aspherical bubble collapse - comparison with simulations. AIP Conference Proceedings 524, pp. 393-396. DOI: $10.1063 / 1.1309249$.

OpenCFD-team and Jozsef Nagy (2017). OpenFOAM: User Guide v2006. accessed Nov. 2020, URL: openfoam.com/documentation/guides/latest/doc/guide-bcs-outlet-pressure-inlet-outlet.html.

Peters, Andreas (2019). Numerical Modelling and Prediction of Cavitation Erosion Using EulerEuler and Multi-Scale Euler-Lagrange Methods. English. PhD-thesis. Universität DuisburgEssen.

Philipp, A. and W. Lauterborn (1998). Cavitation erosion by single laser-produced bubbles. Journal of Fluid Mechanics 361, pp. 75-116.

Pishchalnikov, Y. A., W. M. Behnke-Parks, K. Schmidmayer, K. Maeda, T. Colonius, T. W. Kenny, and D. J. Laser (2019). High-speed video microscopy and numerical modeling of bubble dynamics near a surface of urinary stone. Journal of the Acoustical Society of America 146.516. DOI: $10.1121 / 1.5116693$.

Plesset, M. S. and R. B. Chapman (1971). Collapse of an initially spherical vapour cavity in the neighbourhood of a solid boundary. Journal of Fluid Mechanics 47, pp. 283-290.

Reuter, Fabian and Robert Mettin (2016). Mechanisms of single bubble cleaning. Ultrasonics Sonochemistry 29, pp. 550 -562. DOI: 10.1016/j.ult sonch.2015.06.017.

Reuter, Fabian, Silvestre Roberto Gonzalez-Avila, Robert Mettin, and Claus-Dieter Ohl (2017a). Flow fields and vortex dynamics of bubbles collapsing near a solid boundary. Phys. Rev. Fluids 2, p. 064202. DOI: 10.1103/PhysRevFluids.2.064202.

Reuter, Fabian, Sonja Lauterborn, Robert Mettin, and Werner Lauterborn (2017b). Membrane cleaning with ultrasonically driven bubbles. Ultrasonics Sonochemistry 37, pp. $542-560$. DOI: $10.1016 / j \cdot u l t$ sonch.2016.12.012.

Rosselló, J. M., D. Dellavale, and F. J. Bonetto (2016). Positional stability and radial dynamics of sonoluminescent bubbles under bi-harmonic driving: Effect of the high-frequency component and its relative phase. Ultrasonics Sonochemistry 31, pp. 610-625. 
Rosselló, Juan Manuel, Werner Lauterborn, Max Koch, Tobias Wilken, Thomas Kurz, and Robert Mettin (2018). Acoustically Induced Bubble Jets. Physics of Fluids 30, p. 122004. DOI: $10.1063 / 1.5063011$.

Sperry, J. S., N. Z. Saliendra, W. T. Pockman, H. Cochard, P. Cruiziat, S. D. Davis, F. W. Ewers, and M. T. Tyree (1996). New evidence for large negative xylem pressures and their measurement by the pressure chamber method. Plant, Cell and Environment 19, pp. 427-436.

Stuecker, Malte F., Cecilia M. Bitz, Kyle C. Armour, Cristian Proistosescu, Sarah M. Kang, Shang-Ping Xie, Doyeon Kim, Shayne McGregor, Wenjun Zhang, Sen Zhao, Wenju Cai, Yue Dong, and Fei-Fei Jin (2018). Polar amplification dominated by local forcing and feedbacks. Nature Climate Change 8, pp. 1076-1081. DOI: 10.1038 /s 41558-018-0339-y.

Supponen, Outi, Danail Obreschkow, Marc Tinguely, Philippe Kobel, Nicolas Dorsaz, and Mohamed Farhat (2016). Scaling laws for jets of single cavitation bubbles. Journal of Fluid Mechanics 802,pp. 263-293. DOI: 10.1017/jfm.2016.463.

Söhnholz, Hendrik (2016). Temperatureffekte bei der lasererzeugten Kavitation (Temperature effects at the laser generated cavitation. German. PhD-thesis. Universität Göttingen.

Tryggvason, G., B. Bunner, A. Esmaeeli, D. Juric, N. Al-Rawahi, W. Tauber, J. Han, S. Nas, and Y.-J. Jan (2001). A Front-Tracking Method for the Computations of Multiphase Flow. Journal of Computational Physics 169, pp. 708-759.

Vogel, A. and W. Lauterborn (1988). Acoustic transient generation by laser-produced cavitation bubbles near solid boundaries. Journal of the Acoustical Society of America 84.2, pp. 719 -731 .

Vogel, A and V. Venugopalan (2011). Pulsed laser ablation of tissue. 2nd ed. Springer, Heidelberg, New York.

Vogel, A., J. Noack, G. Hüttman, and G. Paltauf (2005). Mechanisms of femtosecond laser nanosurgery of cells and tissues. Appl. Phys. B 81, pp. 1015-1047. DOI: 10.1007 / s $00340-$ $005-2036-6$.

Vogel, Alfred (1987). Optische und akustische Untersuchungen der Dynamic lasererzeugter Kavitationsblasen nahe fester Grenzflächen (Optical and acoustical investigations of the dynamics of laser generated cavitation bubbles close to rigid boundaries). German. PhD-thesis. Universität Göttingen.

Voinov, O. V. and V. V. Voinov (1976). On the process of collapse of a cavitation bubble near a wall and the formation of a cumulative jet. Sov. Phys. Dokl 21, pp. 133-135.

Wang, Qianxi (2016). Local energy of a bubble system and its loss due to acoustic radiation. J. Fluid Mech. 797, pp. 201-230.

Weller, H. G. (2008). A new approach to VOF-based interface capturing methods for incompressible and compressible flow. Technical Report TR/HGW/04. OpenCFD.

Weller, H. G., G. Tabor, H. Jasak, and C. Fureby (1998). A tensorial approach to computational continuum mechanics using object-oriented techniques. Computers in Physics 12, pp. 620631.

Wikimedia: Davidhv22 (2011). Cavitacion.jpg. image not altered, license: CC-4.0 (creativecommons.org/licenses/by-sa/4.0/deed.en).

Wikimedia: Erik Axdahl (Liftarn) (2007). Cavitation Propeller Damage.JPG. image not altered, license: CC-2.5, (creativecommons.org/licenses/by-sa/2.5/deed.en).

Young, F. R. (1999). Cavitation. London: Imperial College Press. 


\section{A Appendix - Fluid properties}

The standard values applied in this thesis are given in the following table:

\begin{tabular}{lllll} 
Symbol & meaning & value & value used & unit \\
\hline \hline air: & & & & \\
\hline$p_{V}$ & vapour pressure & 2337 & 0 & $\mathrm{~Pa}$ \\
$\mathrm{R}_{\text {general }}$ & general gas const. & 8.3144621 & 8.3144621 & $\mathrm{~J} /(\mathrm{K} \cdot \mathrm{mol})$ \\
$\mathrm{R}_{\text {spec }}$ & gas const. & 287.058 & 287.0 & $\mathrm{~J}(\mathrm{~K} \cdot \mathrm{kg})$ \\
$\sigma$ & surface tension & 0.0725 & 0.0725 & $\mathrm{~Pa} \cdot \mathrm{s}$ \\
$\beta$ & normalized co-volume & $\approx 0.00154$ & 0 & - \\
$\gamma$ & polytropic exponent & 1.4 & 1.4 & - \\
$\mu_{g}$ & dynamic viscosity & 18.6 & 0 & $\mu \mathrm{Pa} \cdot \mathrm{s}$ \\
\hline water: & & & & $\mathrm{K}$ \\
\hline$\vartheta_{0}$ & ambient temperature & 293.15 & 293.15 & $\mathrm{~Pa} \cdot \mathrm{s}$ \\
$\mu_{l}$ & dynamic viscosity & 0.001002 & 0.001002 & $\mathrm{bar}$ \\
$B$ & Tait parameter & 3050 & 3050 & $\mathrm{~Pa}$ \\
$p_{\infty}$ & ambient pressure & 101325 & 101315 & $\mathrm{~kg} / \mathrm{m}^{3}$ \\
$\rho_{\infty}$ & ambient density & 998.20608789369 & 998.20608789369 & - \\
$n_{T}$ & Tait exponent & 7.15 & 7.15 & \\
\hline
\end{tabular}

Table A.1: Standard fluid properties of water and air. 


\section{B Appendix - Numerics}

\section{B.1 Grid convergence study and best practice meshing}

Here the grid convergence studies are given that lead to the Crit. 3.2 in Sec. 3.1.4. The bubble in unbounded liquid with initial data

$$
R_{\text {init }}=20 \mu \mathrm{m}, R_{n, 1}=184.1 \mu \mathrm{m}, R_{n, 2}=64 \mu \mathrm{m}, R_{n} \text { reduction in interval }[60 \mu \mathrm{s}, 75 \mu \mathrm{s}],
$$

is investigated. The time from bubble generation $(t=0)$ and the first minimum bubble volume $\left(t\left(V_{\min }\right)\right)$ is taken as the criterion for convergence. In unbounded liquid the duration for expansion and collapse is the same, thus the convergence criterion is called $2 T_{c}$. This quantity behaved in an unexpected way with resolution refinement in the first place. Numerous solution control parameters have been studied until finally the solution converged. The Crit. 3.2 derive from the following investigations.

\section{B.1.1 Bubble in unbounded liquid - generating a reference solution}

The necessity to resolve a very steep pressure gradient at $t=0$, when an EDC bubble is set up, makes grid convergence studies for axisymmetric calculations an everlasting task. Therefore, in order to show the convergence of the method, the grid study was performed for a bubble in unbounded liquid, which can also be done in spherical symmetry, thus reducing computational cost even at very high resolutions. All investigations were done with adapting the initial bubble energy according to Eq. 2.22: After discretization, the true bubble volume $V_{0}$ is determined and the values of $p_{0}$ and $R_{n}$ are adapted accordingly to have the same energy as a bubble of $R_{\text {init }}=20 \mu \mathrm{m}, R_{n}=184.1 \mu \mathrm{m}$. All investigations are carried out with a maximum flow Courant number of 0.2 , a maximum Courant number for the interface of 0.08 and maximum acoustic Courant numberof 8 for the whole time domain. The maximum acoustic Courant number is reduced to 1 if the bubble equivalent radius is below $30 \%$ of $R_{n}$. The upper size of the time step is $\Delta t=5 \cdot 10^{-8} \mathrm{~s}$ and the time step size at $t=0$ is set to $\Delta t_{0}=10^{-11} \mathrm{~s}$.

Figure B. 1 shows the first attempt with a boundary distance of $\mathrm{XF}=80 R_{\max }$. The spatial resolution is varied in the initial bubble domain for both axisymmetric and spherically symmetric calculations with Mesh 3.1.2.a and Mesh 3.1.2.b (version A), respectively. For $t=0$ the bubble interface (int.f.) was either set up with a thickness of 3 cells or sharp with approximately zero thickness in theory. Both meshes use the same dimensional parameters. The core size $\mathrm{C}$ of both meshes was set to $80 \mu \mathrm{m}$ and $\mathrm{X}$ is set to $1.2 R_{\max }$. It is seen that the bubble shows the same collapse time for the same method in each of the applied mesh symmetries. But for very high resolutions a random behaviour seems to occur. The shockwave of the bubble generation travels to the boundary, located at a distance of $80 R_{\max }=80 \cdot 495 \mu \mathrm{m}=39.6 \mathrm{~mm}$, and a reflected part travels back to the bubble within approximately $54.6 \mu$ s, assuming a uniform sound speed of $1450 \mathrm{~m} / \mathrm{s}$. During this time, the bubble reaches maximum expansion and a state of low pressure 


\section{B Appendix - Numerics}

and low interface velocity. In this state it is supposed that it is influencable by impinging waves. The higher the resolution, the sharper the shockwave is resolved, thus leading to higher influence.
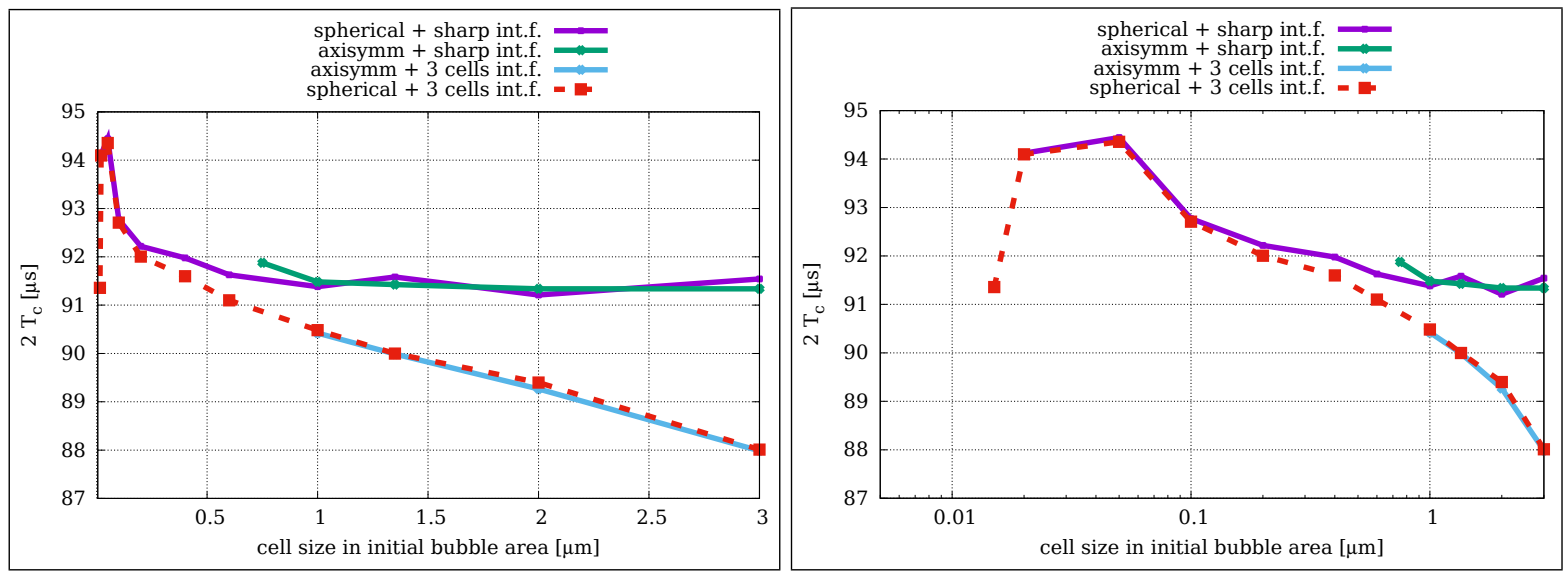

Fig. B.1: Time from bubble generation till first minimum volume for different resolutions for an unbounded bubble in both spherical and axial symmetry with a outer boundary distance of $80 R_{\max }$. For very high resolutions suddenly a non-converging, random behaviour appears, irrespective whether the bubble interface is smeared over 3 cells at $t=0$ or not. The lower curves are the ones with smeared interface. The upper curves use a sharp interface in the beginning. Left and right diagrams are the same but linear or logarithmic $x$-axis.

In order to show that most probably no other parameter than the boundary distance is capable of enhancing convergence, the global mass correction $(G M C)$ and also zero surface tension $($ sigma $=0)$ were tested in Fig. B.2, thereby validating that the local mass correction $(L M C)$ produces the same collapse times as the global mass correction. The GMC was tested and validated in Koch et al. (2016).
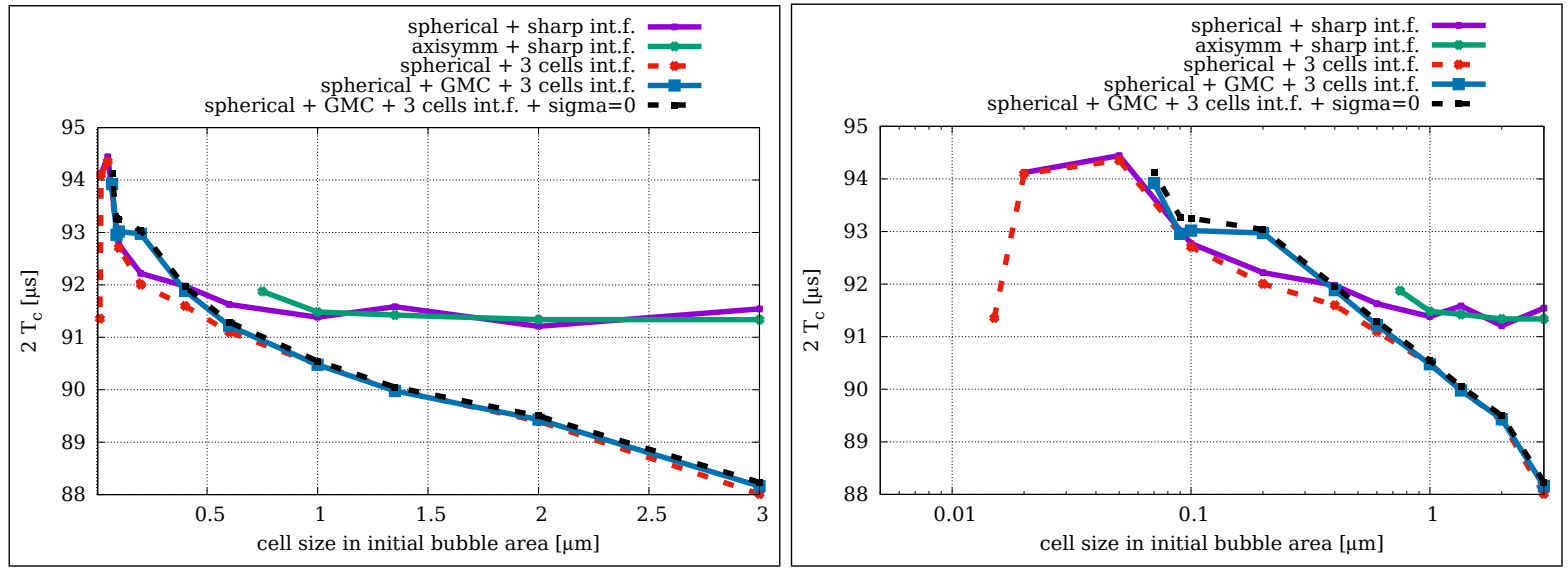

Fig. B.2: Same investigation as Fig. B.1 but including investigations where the global mass correction (GMC) was applied to check for better convergence. This, however is not the case. Also, the surface tension $\sigma$ was supposed to have an influence, which turned out negligible. 

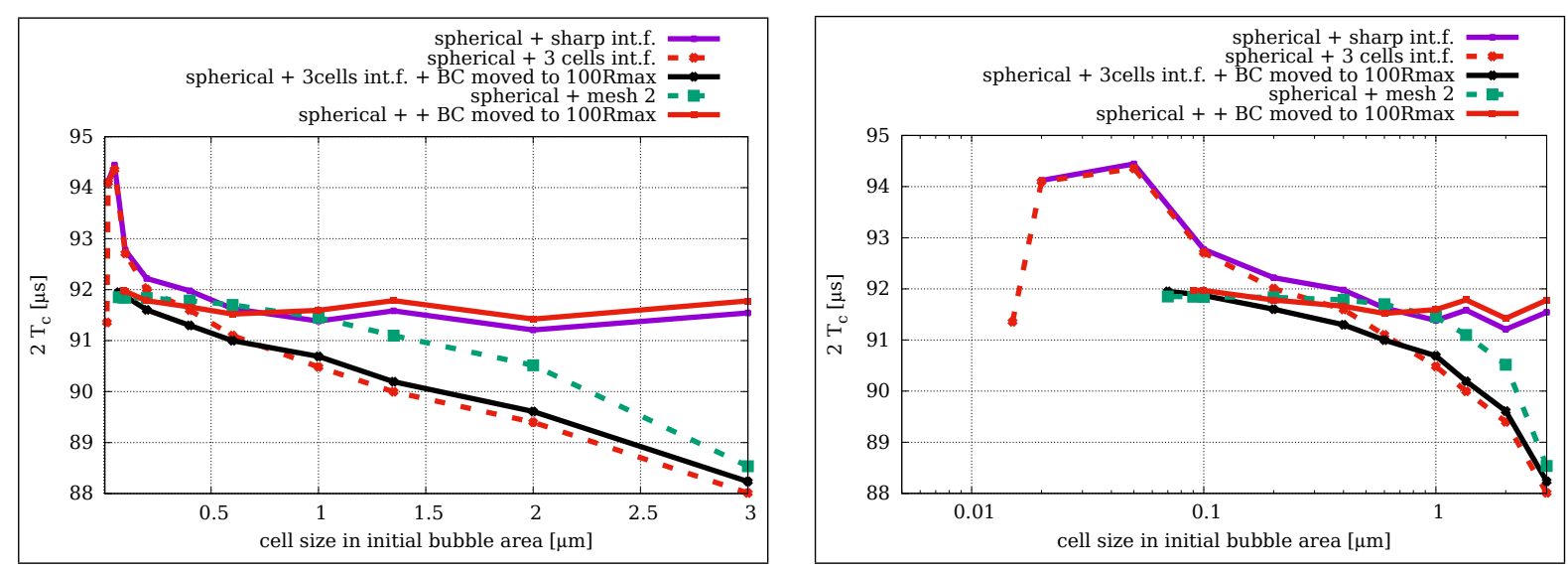

Fig. B.3: Same investigation as Fig. B.1 finally having found convergence for setting the outer boundary from $80 R_{\max }$ to $100 R_{\max }$ apart from the bubble. Mesh 2 refers to Mesh 3.1.2.b (version B) with parameters: $\mathrm{X}=20 \mu \mathrm{m}=R_{\text {init }}, \mathrm{XF}=R_{\max }$, $\mathrm{XFF}=20 R_{\max }, \mathrm{XFFF}=200 R_{\max }, \theta=1^{\circ}$.

Finally in Fig. B.3, convergence was found by testing the spherical Mesh 3.1.2.b version $\mathrm{B}$ (mesh 2) with a boundary distance of XFFF $=200 R_{\max }$. This led to testing Mesh 3.1.2.b version A with a boundary distance of $\mathrm{XF}=100 R_{\max }$, improving convergence for almost every resolution for the case of an initially sharp interface (red curve). Indeed, it can be seen in comparison with Fig. B.1, that even with a boundary distance of only $80 R_{\max }$ the axisymmetric case with a sharp interface at $t=0$ is congruent with the spherical solution that converges. The congruence is best in the resolution interval from $1 \mu \mathrm{m}$ to $3 \mu \mathrm{m}$.

The curves of the equivalent Radius over time for the converged and non-converged series are given in Fig. B.4. It is seen that the rebound radius is underestimated for the resolutions $3 \mu \mathrm{m}$ and $2 \mu \mathrm{m}$. So it can be expected that the solution converges fully from $1.35 \mu \mathrm{m}$ onward.

The axisymmetric calculation (green curve in Fig. B.1) is in congruence with the converged spherical calculation even though the boundary distance was $80 R_{\max }$. This is not a contradiction, because the resolutions where non-convergence is observed are higher than the ones that could be tested within reasonable time.

One has to note, too, that the above grid convergence study aims at extremely high precision. Even the worst non-converging solution of Fig. B.1 has roughly a maximum deviation in twice the collapse time of $4 \mu$ s from the value of $92 \mu$ s, which is considered the true one. This results in a maximum relative error $\epsilon_{r \text {,max }}$ of

$$
\epsilon_{r, \max }=\frac{4 \mu \mathrm{s}}{92 \mu \mathrm{s}}=4.3 \%
$$

This is still below $5.5 \%$ which was the precision considered sufficient in Koch et al. (2016) for similar studies.

The two reference solutions are summarized in Fig. B.5. 


\section{B Appendix - Numerics}
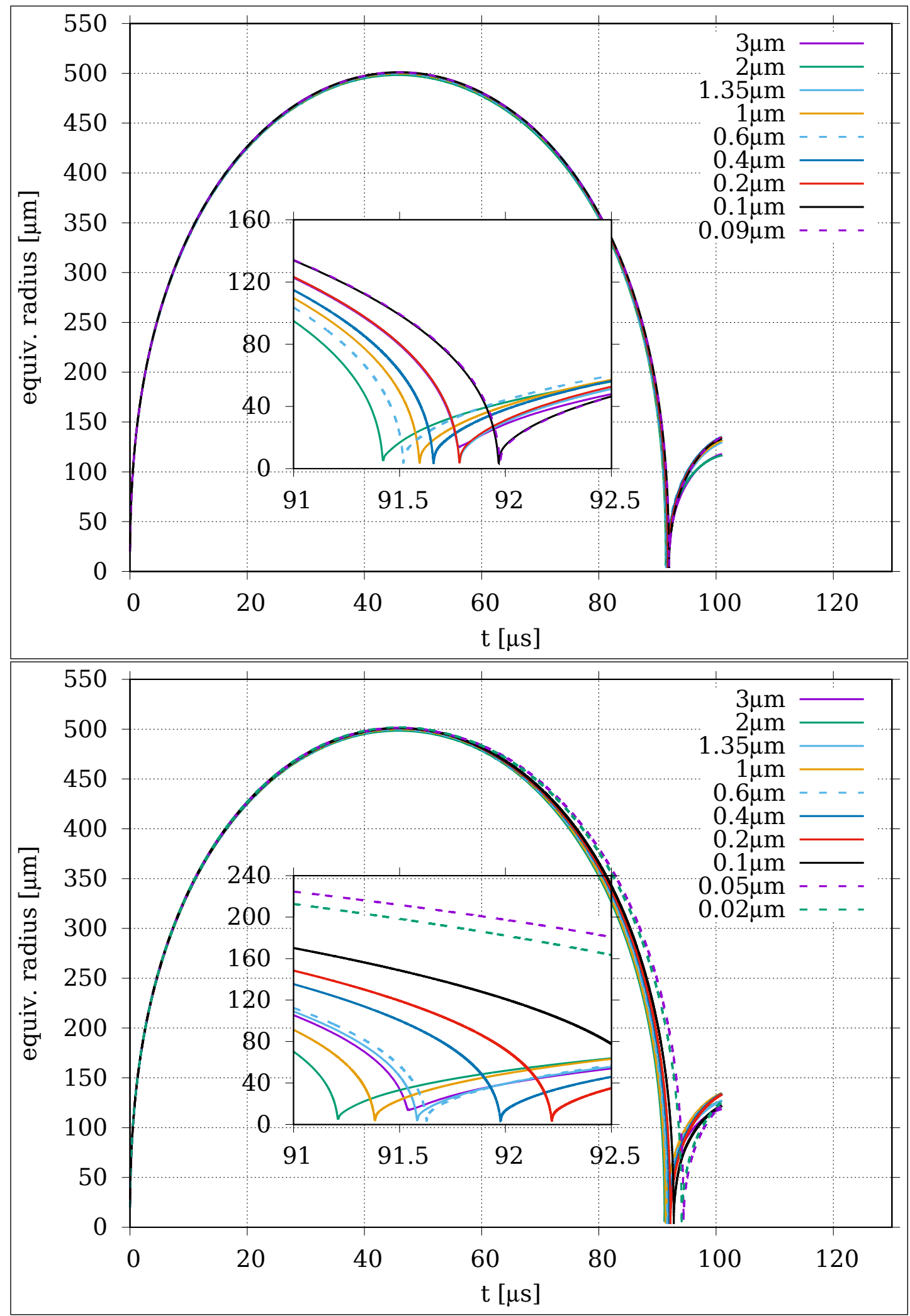

Fig. B.4: Equivalent radius over time for the converged series (top) of Fig. B.3 and non-converged series (bottom). Both with sharp interface. 


\section{B Appendix - Numerics}

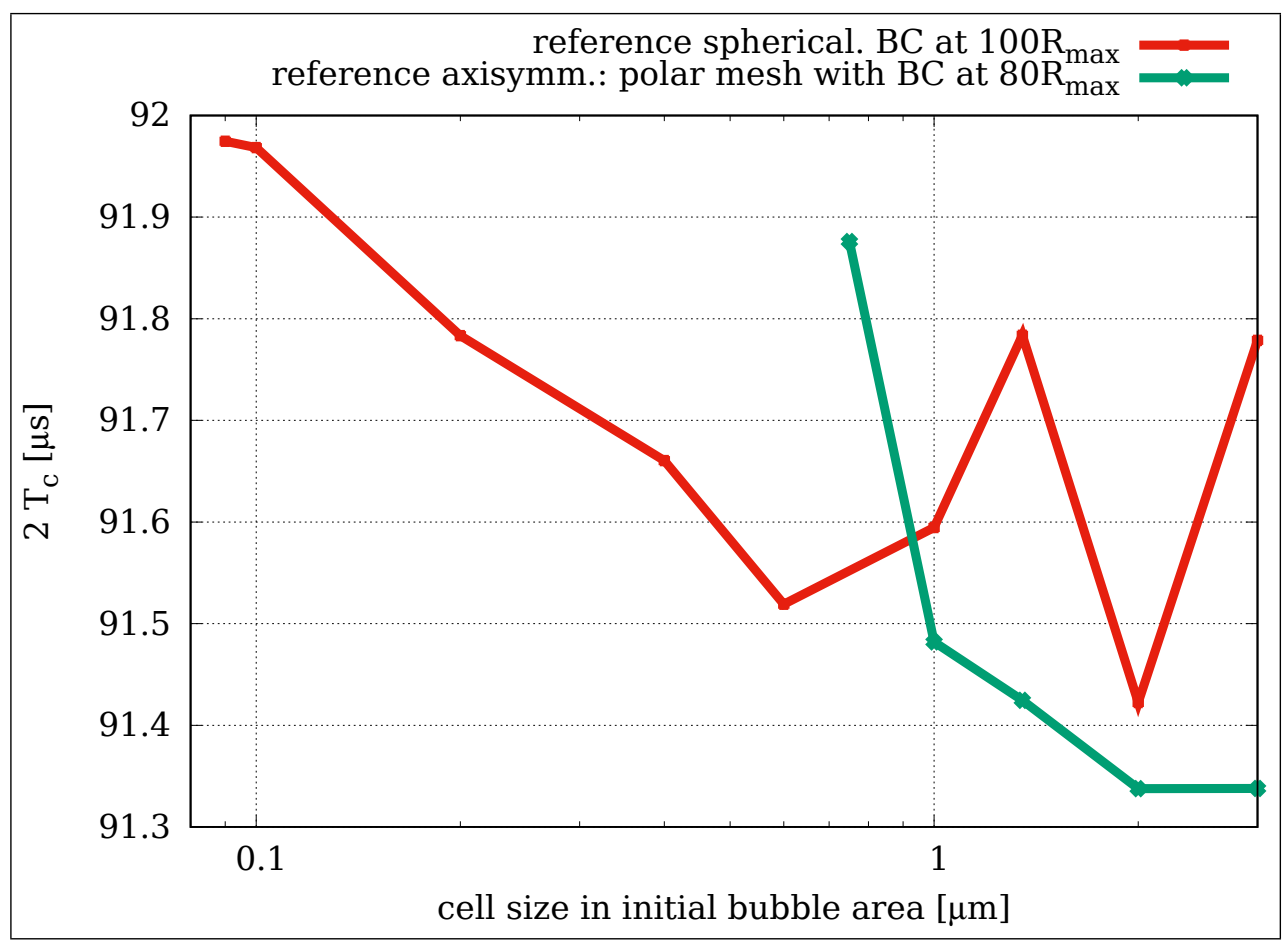

Fig. B.5: Time from bubble generation till first minimum volume for different resolutions for the two reference solutions for the unbounded bubble. Axisymmetric calculation (green) of Fig. B.1 and converged spherical calculation (red) of Fig. B.3. This Figure is the same as Fig. 3.8

\section{B.1.2 Words on saving calculation time}

Calculation time is an issue. The following considerations concern the software side and hold for 1D, 2D and also 3D. The hardware side is covered in the Appendix Ch. B.4.

Figure B.6 (left) shows the calculation times in logarithmic scale over the resolution for the axisymmetric reference solution of Fig. B.5. Considering the convergence studies of Sec. B.1.1, a resolution of $1 \mu \mathrm{m}$ would be preferred for axisymmetric calculations, but approximately one and a half hour calculation time on a 16 core AMD Ryzen machine still limits the parameter space of inquiry. At this point, the space is open for optimization. Regarding the mesh, one can now play with the geometric parameters. That means the grading values or the positions of the $X F_{i}$ for the polar mesh of the reference solution. The aim is to reduce the amount of cells in a most clever way and save calculation time by matrix size reduction. The geometric configuration of the reference bubble studied was:

$$
\mathrm{Xi}=80 \mu \mathrm{m}, \mathrm{Xii}=1.2 \cdot \sqrt{2} \cdot \mathrm{Xi}, \mathrm{X}=1.2 R_{\max }, \mathrm{XF}=80 R_{\max }, g=5.25 .
$$

The other possibility is to go for a different meshing approach, e.g. the static refinement Mesh 3.1.2.c. The latter has been rediscovered only recently and therefore still needs a lot of validation work. In principle, it was found to be able to reduce the calculation time by a factor of 2.51 as shown in Fig. B.6 (right), when the maximum Courant number for the interface is set to 0.2 rather than 0.08 , too. However, in the case of a bubble close to a solid boundary it was found to be responsible for a very different jet shape at resolutions of $1 \mu \mathrm{m}$ and coarser. This will be shown in Sec. B.1.3. The conclusion is that the influence of the borders between the 


\section{B Appendix - Numerics}

concentric refinements, where the resolution jumps by a factor of two within one cell distance, is not yet fully understood.

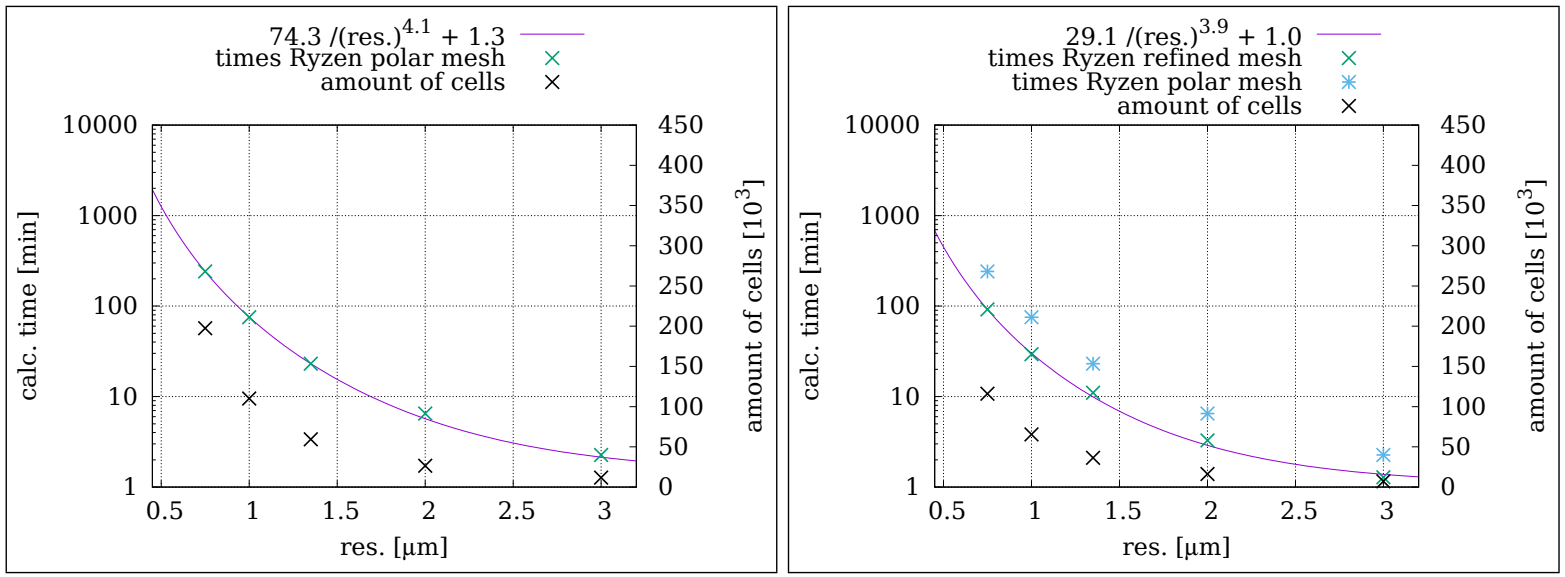

Fig. B.6: Calculation times (16 threads on Ryzen machine) for left: the axisymmetric reference solution in Fig. B.5, and right: same calculation but on a mesh with static refinement.

This conclusion persists for another time saving approach: The adaptive mesh refinement. Thanks to Luca Cornolti (presumably author of Cornolti et al. (2013), user Luca Cornolti on cfdonline.com) this method is also available and working stably for calculations in axial symmetry. According to user-defined thresholds of a chosen field the mesh is refined every $n$-th timestep in the area where the thresholds are crossed. The method was found to drastically reduce calculation time, because only the interface is kept at high spatial resolution, however it also challenges numerical stability. There is still a lot of validation work to be done since it was found to lead to different solutions than a static mesh in some tryouts.

After optimizing the mesh, the time stepping can be optimized: As mentioned above, it is possible to raise the maximum Courant number for the interface to 0.2 rather than 0.08 . However, it requires to restrict the maximum acoustic Courant number for the whole time domain. A value of 8 guarantees stability in most of the cases, while from about a value of 30 instabilities may occur. The values were not tested explicitly, but got consolidated on the way.

\section{B.1.3 Bubble close to a planar solid boundary}

In Sec. B.1.1 it was shown that using the bubble collapse time of an unbounded bubble as the criterion for studying solution convergence, simulating a cavitation bubble becomes very demanding. The solution converges earliest from a resolution of $1.35 \mu \mathrm{m}$ in the initial bubble area onward if the best practice guidelines for the mesh are fulfilled. The conclusion was that for simulations in axial symmetry the preferred resolution is a cell size of $1 \mu \mathrm{m}$.

In this section however, it is shown that taking the bubble shape as a convergence criterion, loosening the temporal precision claims, convergence is found much earlier. This will be explained with a bubble close to a solid boundary, with the following theoretical parameters:

$$
\begin{aligned}
R_{\text {init }}=20 \mu \mathrm{m}, R_{n, 1} & =184.1 \mu \mathrm{m}, R_{\text {max,unbound }}=495 \mu \mathrm{m}, D_{\text {init }}=792 \mu \mathrm{m} \rightarrow D^{*}=1.6 \\
R_{n, 2} & =64 \mu \mathrm{m}, R_{n} \text { reduction in interval }[60 \mu \mathrm{s}, 75 \mu \mathrm{s}]
\end{aligned}
$$

This bubble is simulated with the axisymmetric, polar Mesh 3.1.2.a of the following parameters:

$$
\mathrm{Xi}=50 \mu \mathrm{m}, \mathrm{Xii}=2 \cdot \mathrm{Xi}, \mathrm{X}=1.05 R_{\max }, \mathrm{XF}=100 R_{\max }, g_{f}=5.2
$$




\section{B Appendix - Numerics}

The cell size is sampled with values:

$$
\Delta x \in[0.75 \mu \mathrm{m}, 1 \mu \mathrm{m}, 1.35 \mu \mathrm{m}, 2 \mu \mathrm{m}, 3 \mu \mathrm{m}]
$$

The initial data is corrected by algorithm 2 of Tab. 3.1.

Figure B. 8 shows the bubble interface contours for three subsequent times before the jet impacts onto the opposite bubble surface. It is seen that the bubble shape is quite correct even for the $3 \mu \mathrm{m}$ resolution and perfectly matching from $2 \mu \mathrm{m}$ on.

If the spatial convergence is that good, the question arises how well quantities converge that are time dependent, too. Therefore, the maximum liquid jet velocity $v_{j}$ (= minimum liquid velocity due to negative values) into the direction towards the solid boundary along the $y$-axis (= axis of symmetry) is investigated.

$$
v_{j}=\left.\min \left(\alpha_{1} \mathbf{U} \cdot \mathbf{e}_{y}\right)\right|_{x=0}
$$

The time dependence of this quantity is shown in Fig. B.7. It is seen that the magnitude of the jet velocity increases from $90 \mu$ s to approximately $98 \mu$ s in similar manner irrespective of resolution. However, during the time of minimum $v_{j}$ peaks and jumps occur, so that it seems to be a matter of taste which single value of $\min \left(v_{j}\right)$ to choose. Therefore it is necessary to define what is understood as jet velocity:

Jet velocity is understood here as the maximum magnitude of the $y$-component of the liquid velocity directed towards the solid boundary along the axis of symmetry during the jetting time of the bubble, excluding pressure wave and shockwave contributions.

With this definition it is necessary to know whether the peak velocity in the graphs of Fig. B.7 truly roots from plain liquid flow.
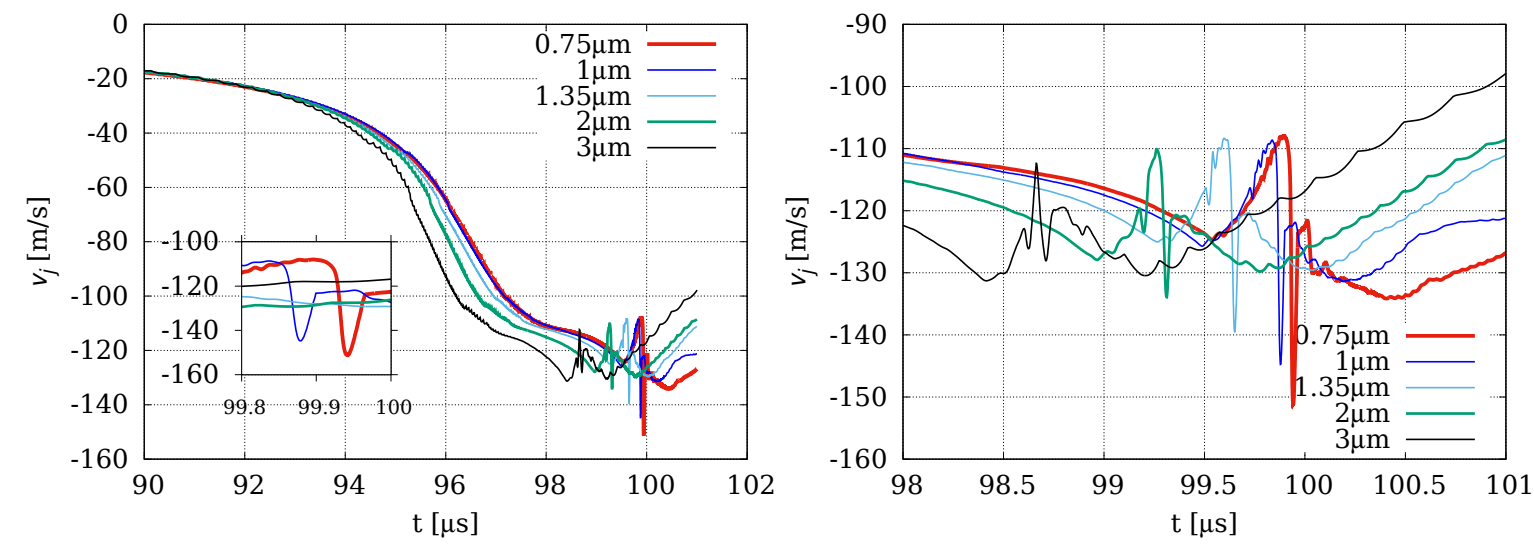

Fig. B.7: Grid convergence of the liquid jet velocity $v_{j}$ (Eq. B.2) for the bubble with properties B.1. Left and right show the same curves for different time intervals 


\section{B Appendix - Numerics}
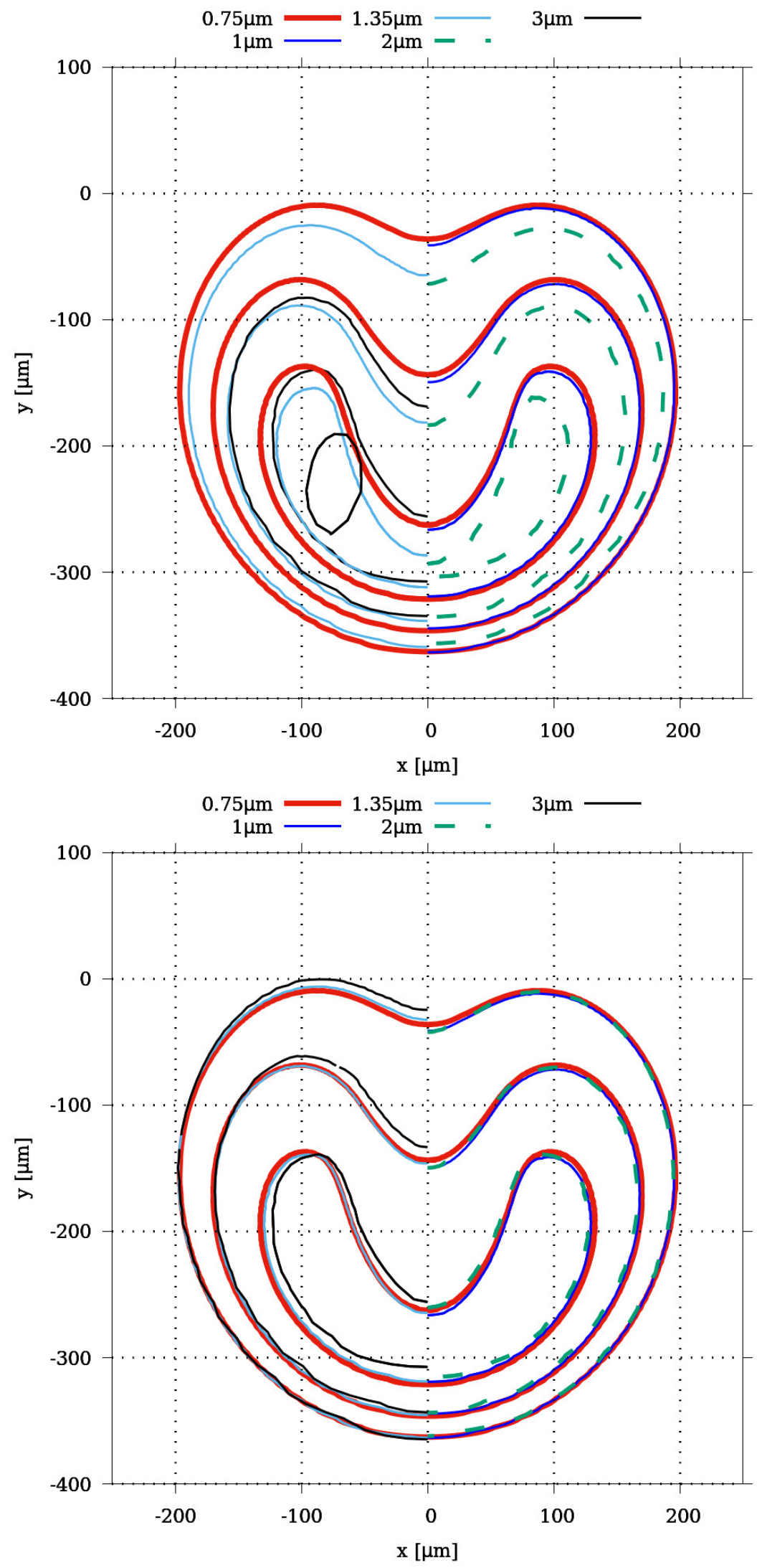

Fig. B.8: Convergence of the bubble shape for a bubble of $D^{*}=1.6$ shown for three time instants before jet impact. Axisymmetric, polar Mesh 3.1.2.a. Top: same times for all resolutions: $t_{i}=97 \mu \mathrm{s}, 98 \mu \mathrm{s}, 99 \mu \mathrm{s}$; Bottom: corrected times for $3 \mu \mathrm{m}$ : $t=t_{i}-1.2 \mu \mathrm{s}$, for $2 \mu \mathrm{m}$ and $1.35 \mu \mathrm{m}: t=t_{i}-0.3 \mu \mathrm{s}$ 


\section{B Appendix - Numerics}
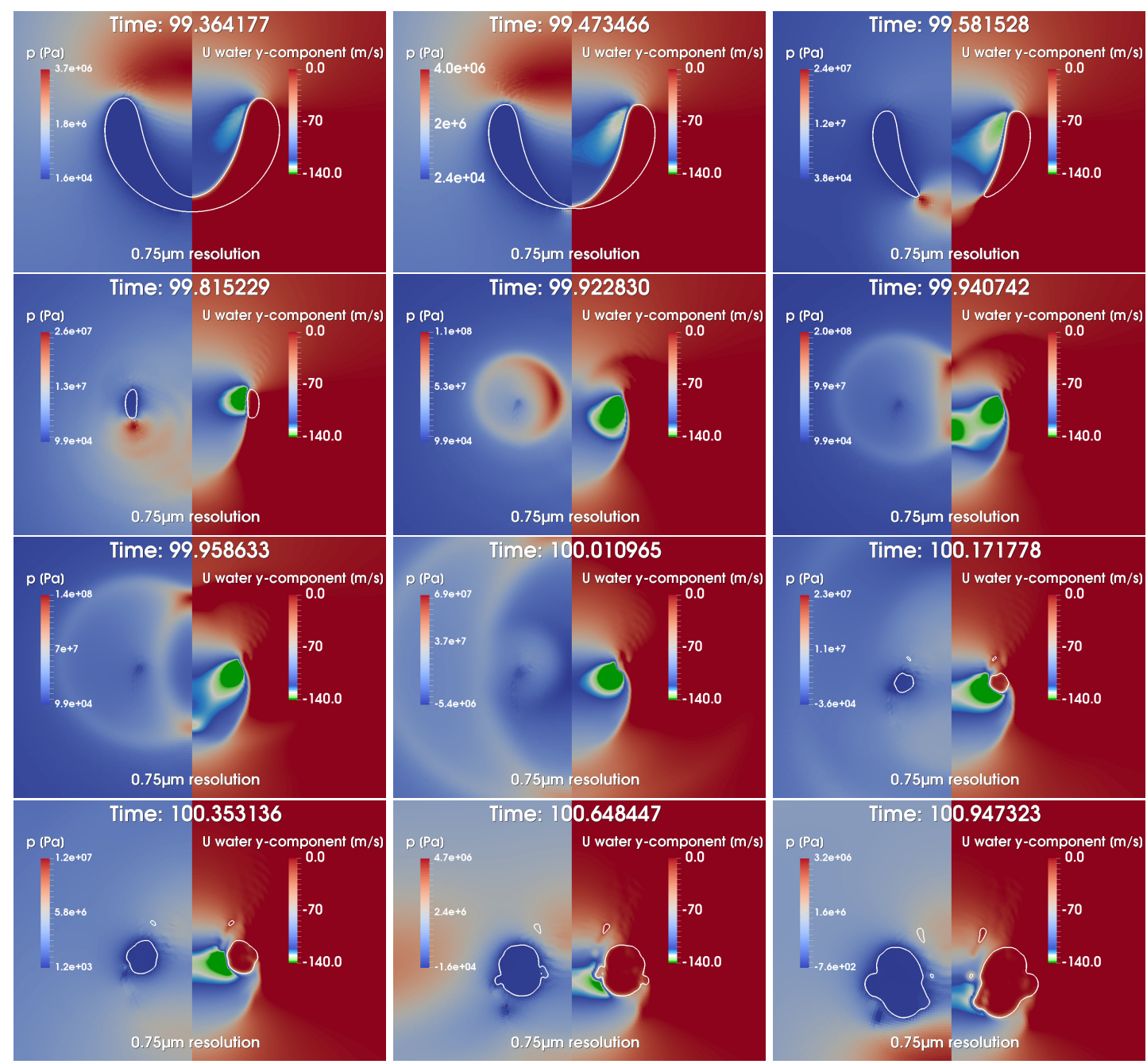

Fig. B.9: Temporal evolution of the liquid jet in the $0.75 \mu \mathrm{m}$ calculation. Left part of the frames shows the color coded pressure in pascal re-scaled for every frame, the right part shows the clipped $y$-component of the liquid velocity $\left(\alpha_{1} \mathbf{U} \cdot \mathbf{e}_{y} \in[-140,0] \mathrm{m} / \mathrm{s}\right)$

Figure B.9 shows the time evolving of the liquid jet at $0.75 \mu \mathrm{m}$ resolution with the pressure in pascal on the left and the $y$-component of the liquid velocity on the right of each frame. The velocity is clipped and given a green color at the lower boundary of the values in order to visualize the region of highest velocity. Mind that $v_{j}$ is evaluated along the axis of symmetry only. One can see that the green area of velocity touches the axis of symmetry in a short period between $99.94 \mu \mathrm{s}$ and $100.01 \mu \mathrm{s}$. This is clearly the peak seen in B.7, e.g. left figure inset. After that the lighter green area touches the axis of symmetry with a decay in green color until the last frame. This is identified as the local minimum (=maximum of magnitude) of $v_{j}$ after the peak. To conclude, the global peak of the $v_{j}$ magnitude stems from the torus shockwave emission, while the local extreme afterwards seems to be a value of pure liquid flow. Therefore, the values for $\min \left(v_{j}\right)$ are chosen as shown in Fig. B.10. 


\section{B Appendix - Numerics}
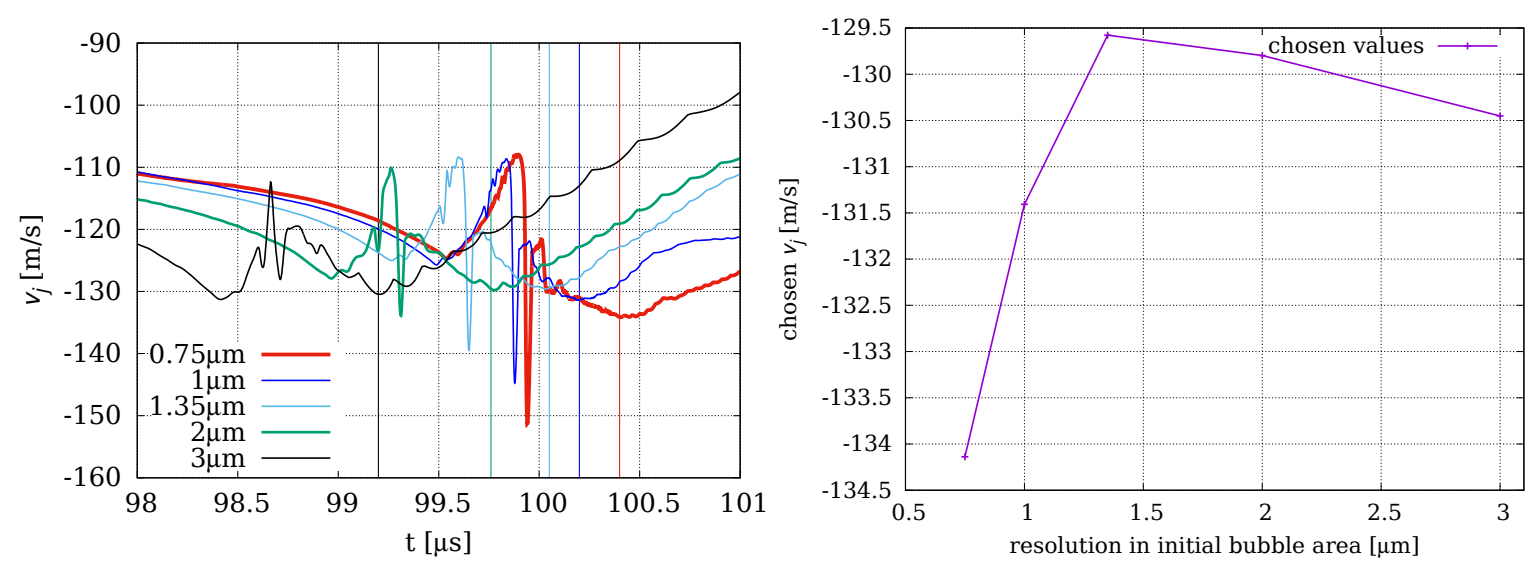

Fig. B.10: Left: repeated diagram of Fig. B.7 but with indicated time values for choosing $v_{j}$. Right: minimum values for $v_{j}$ were extracted within an interval of $0.1 \mu$ s around the chosen values from left diagram.

Interesting to see is that even though the bubble shape of the $1 \mu \mathrm{m}$ and $0.75 \mu \mathrm{m}$ calculation perfectly match in time (see again Fig. B.8), the resulting jet velocity still differs by approximately $2 \%$. All in all, the jet velocity values differ by maximum $3.5 \%$. Whether that is sufficient, is a matter of taste of the scientist. 


\section{B.2 Influence of the surface tension on the mushroom bubbles}

SnappyHexMesh was found to provoke problems for the bubble dynamics in some cases. In the cases of the mushroom investigations, surface tension could not be used, because of instabilities in the velocity in the very cell in the corner between solid surface and axis of symmetry. $U$ got unstable after about 550 time-steps (Fig. B.11).

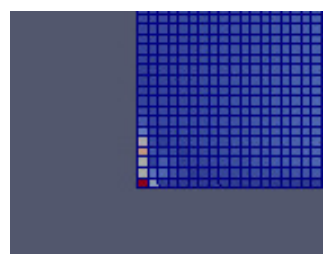

Fig. B.11: SnappyHexMesh induced issue with the surface tension. Velocity instabilities in the corner between axis of symmetry (left edge) and solid surface (lower edge).

The reason could not be found, but it is evident that it has to do with snappyHexMesh because of experience with other cases, too.

The contours of the three $R_{n, 1}$ of Ch. 7 are compared for calculations with and without surface tension:
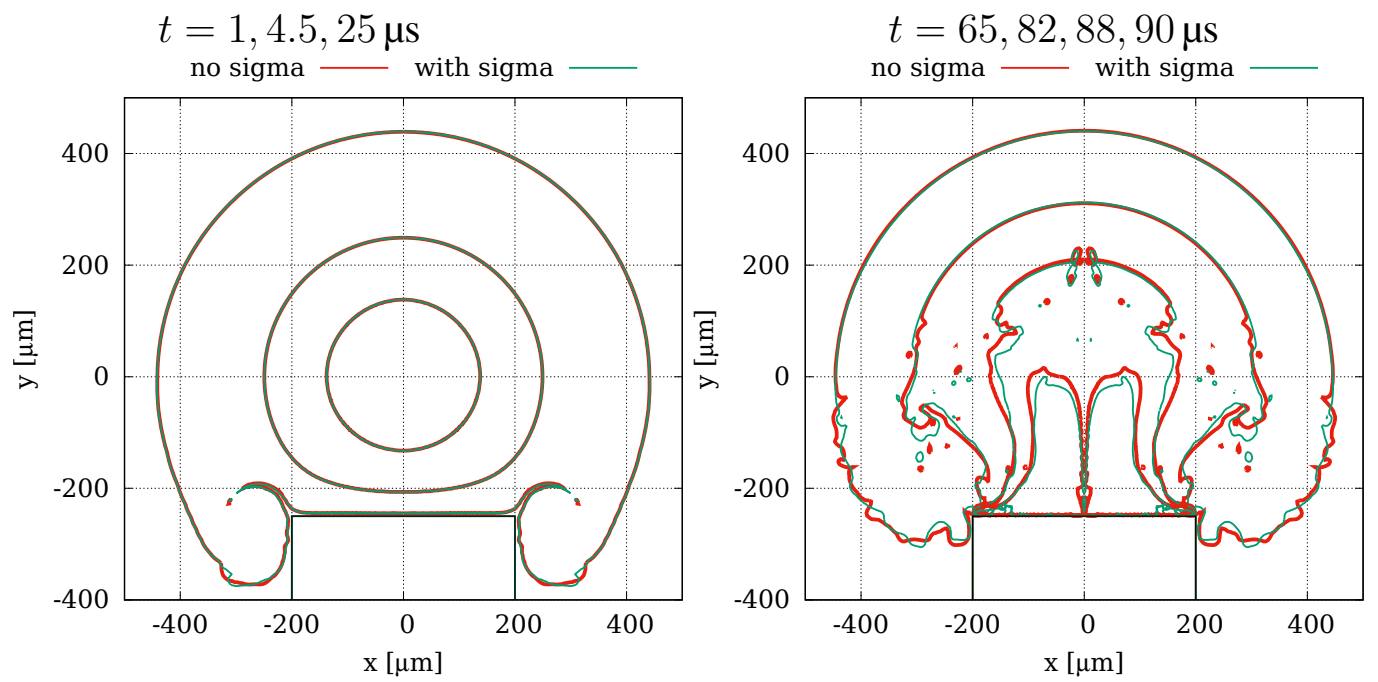

Fig. B.12: Contours of the bubble $R_{n}=184.1 \mu \mathrm{m}, D_{\text {init }}=250 \mu \mathrm{m}, r_{p}=200 \mu \mathrm{m}$. No severe difference is found with or without surface tension up to jet impact. 


\section{B Appendix - Numerics}
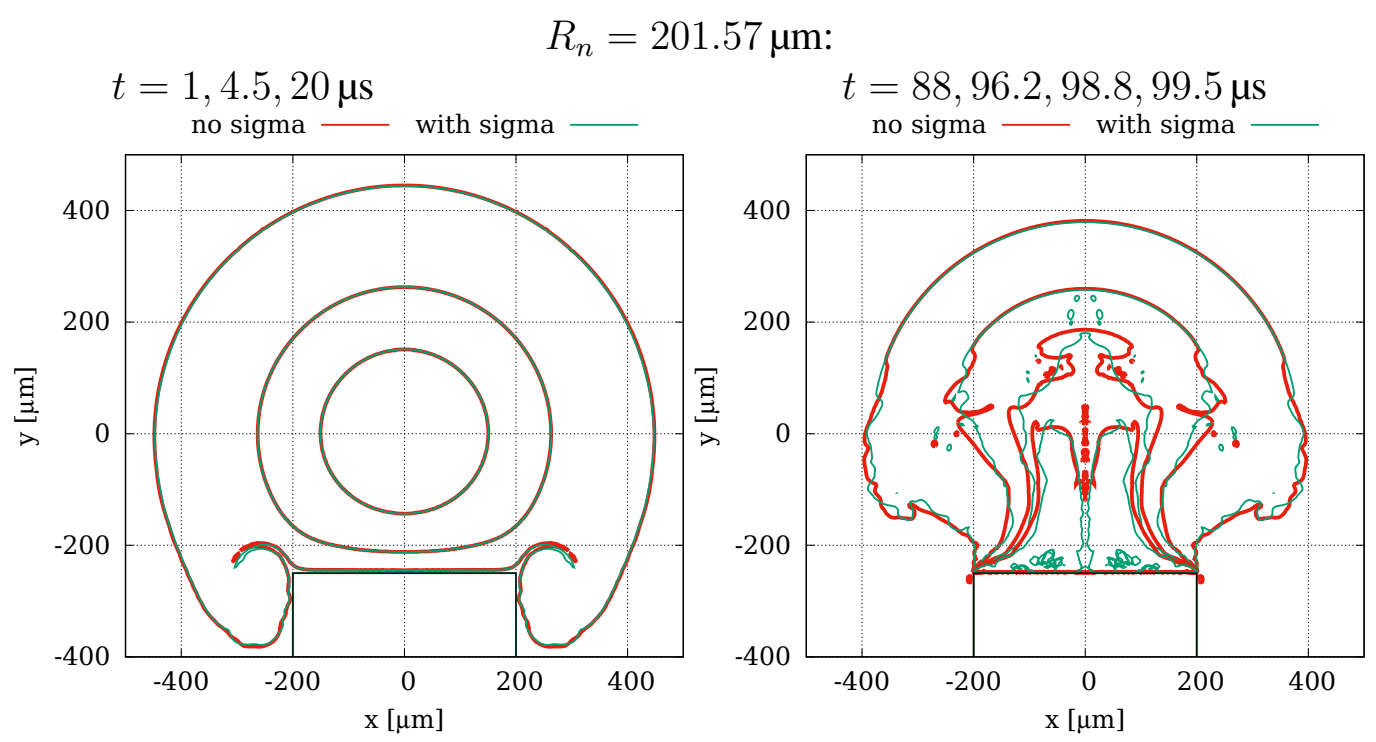

$R_{n}=240 \mu \mathrm{m}:$
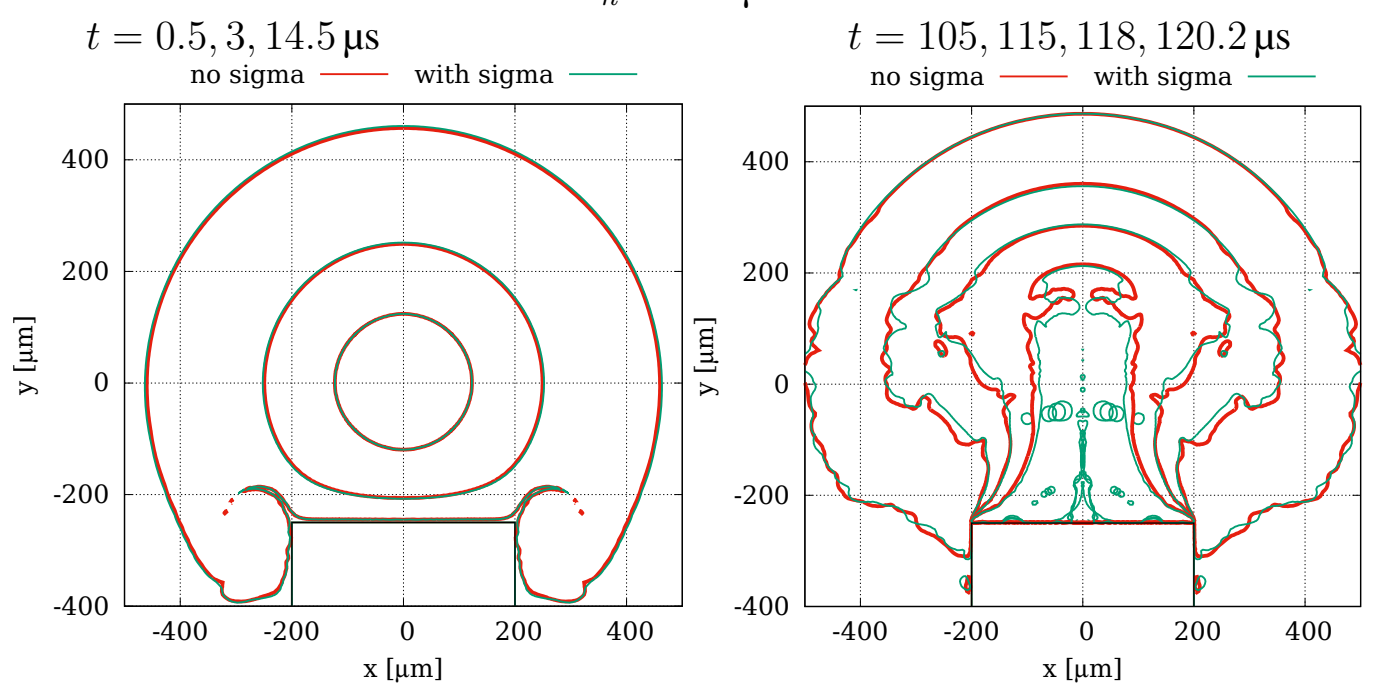

Fig. B.13: Contours of the bubble $R_{n}=201.57 \mu \mathrm{m}$ (top) and $R_{n}=240 \mu \mathrm{m}$ (bottom) with both $D_{\text {init }}=250 \mu \mathrm{m}, r_{p}=200 \mu \mathrm{m}$. The issue shown in Fig. B.11 induces a numerical upwards jet.

\section{B.3 Master-thesis, code and data availability}

Repository B.1. The Master's thesis (Koch 2014) is available here

https://doi.org/10.25625/JVCJVW

In that 175 pages long thesis the equations derivation is very detailed.

This thesis is understood as open source friendly. Most of the source code is available and maintained under the following repositories. The official mirrors offer the versions used for this thesis, maintenance is only done in the private mirrors (github):

Repository B.2. Solver for a cavitation bubble on a static mesh: You can become a collaborator on our github project. If you like to do so, please mail to me 
max.koch@phys.uni-goettingen.de or niob@gmx.li

Repository B.3. many[!] relevant scripts for setting up/ running/ and post-processing a bubble calculation

https://github.com/ma-tri-x/scripts_for_cases

Repository B.4. notes on how to set up ubuntu for foam-extend-4.0 and benchmarking template

$$
\text { https://github.com/ma-tri-x/setup_ubuntu }
$$

The benchmark has been executed by many users in the thread

https://www.cfd-online.com/Forums/hardware/198378-openfoam-benchmarks-various-hardware-14.html

and thus the results are comparable to a lot of other hardware setups

Scripting Paraview and blender with python In order to batch render a bubble over many timesteps it was necessary to apply python scripting to both Paraview and blender. Examples how to do this are found in repository B.3 in the scripts named render_timestep_via_pvsm.py, render_bubble_inside_cuvette_V4.py and run_blender.sh.

\section{B.3.1 Bubble case setup}

The scripts for setting up a bubble case are found in the repository B.3. The main file in a case is the rerun.sh file which is started with

$$
\text { bash rerun.sh -par }
$$

and the main config file is conf_dict.json. Most other files have copy with extension ".template" and are automatically created from this template. Variables declared in the conf_dict.json can be used in the template files via capital letters.

Schemes and solver controls The standard schemes and solver controls used can be found in the cases files of repository B.3.

AMR - Adaptive mesh refinement $\quad$ Has been implemented for 3D and also axial symmetry thanks to the library of Luca Cornolti. However, since it didn't contribute to this thesis and it hasn't been tested sufficiently, it will not be described here. Mail to me (see repository B.2) if you're interested.

\section{B.4 Appendix - Benchmarking different machines}

Summary: The winner of all "low-budget" systems is Berlin, a dual Xeon CPU machine at 2.0 GHz because it exhibits $100 \mathrm{~GB} / \mathrm{s}$ CPU to RAM connection. Tested systems were:

- Kinkade: 16 cores AMD Ryzen 9 3950X, 51.2 GB/s memory bandwidth

- Berlin: $2 \times 8$ cores Xeon E5-2650 1st gen., $2 \times 54.98$ GB/s memory bandwidth 


\section{B Appendix - Numerics}

- Berlin GPU: Nvidia Tesla K20M, 5 GB RAM, 208.0 GB/s memory bandwidth

- Dali: 8 cores Intel i7-9900, 69.148 GB/s memory bandwidth

- Kirchner: 4 cores Intel i7-2600, 21 GB/s memory bandwidth

- Spitzweg: 57 cores at $1.1 \mathrm{GHz}$ Intel Xeon Phi Mic Coprocessor 31S1P, 8 GB RAM, $320 \mathrm{~GB} / \mathrm{s}$ memory bandwidth

- GWDG Cluster: tested up to 300 cores spread over 26 nodes

Dual Xeon E5-2650 vs i7-9900 vs AMD Ryzen 3950X The three most important workstations of this project were tested with the motorbike test case described in repository B.4. The calculation times can be compared to the ones obtained by the community (link given in the repository description). Results are given in Figs. B.14 and B.15.

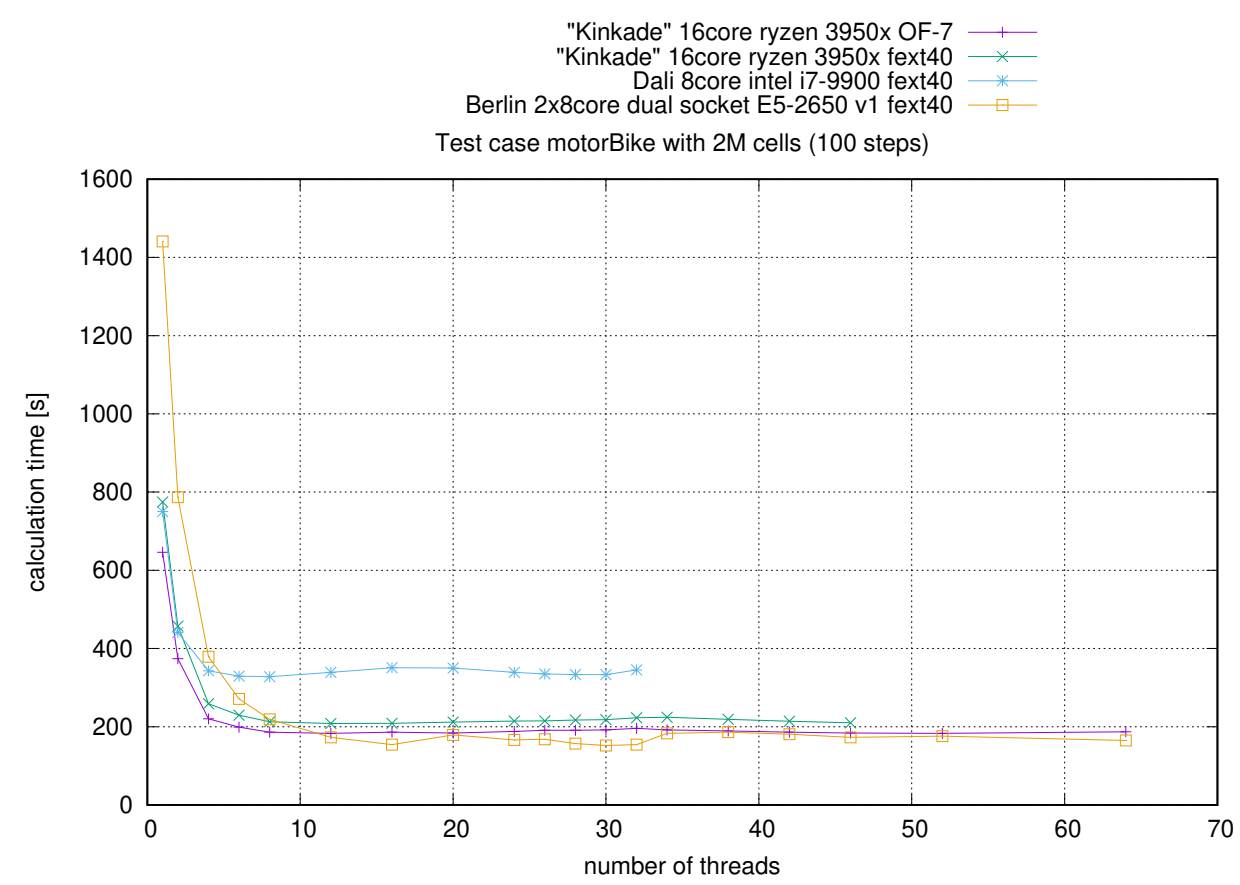

Fig. B.14: Calculation time of the motorbike benchmarking case for different machines.

The same conclusion can be drawn as was found in the community: Computational Fluid Dynamics has its main bottleneck in the memory bandwidth because huge matrices have to be iterated over and over for each time-step. The Xeon-E5 machine has a maximum clock speed of $2.0 \mathrm{GHz}$ while the AMD Ryzen machine has about $4.2 \mathrm{GHz}$ (measured while calculating) and both have the same amount of cores and threads. The reason why the Xeon-E5 machine is still slightly faster, is that the memory bandwidth is twice as high as the one of the AMD Ryzen machine. Furthermore, it can be seen that only the core amount counts, the hyper-threading does not contribute to speedup. 


\section{B Appendix - Numerics}

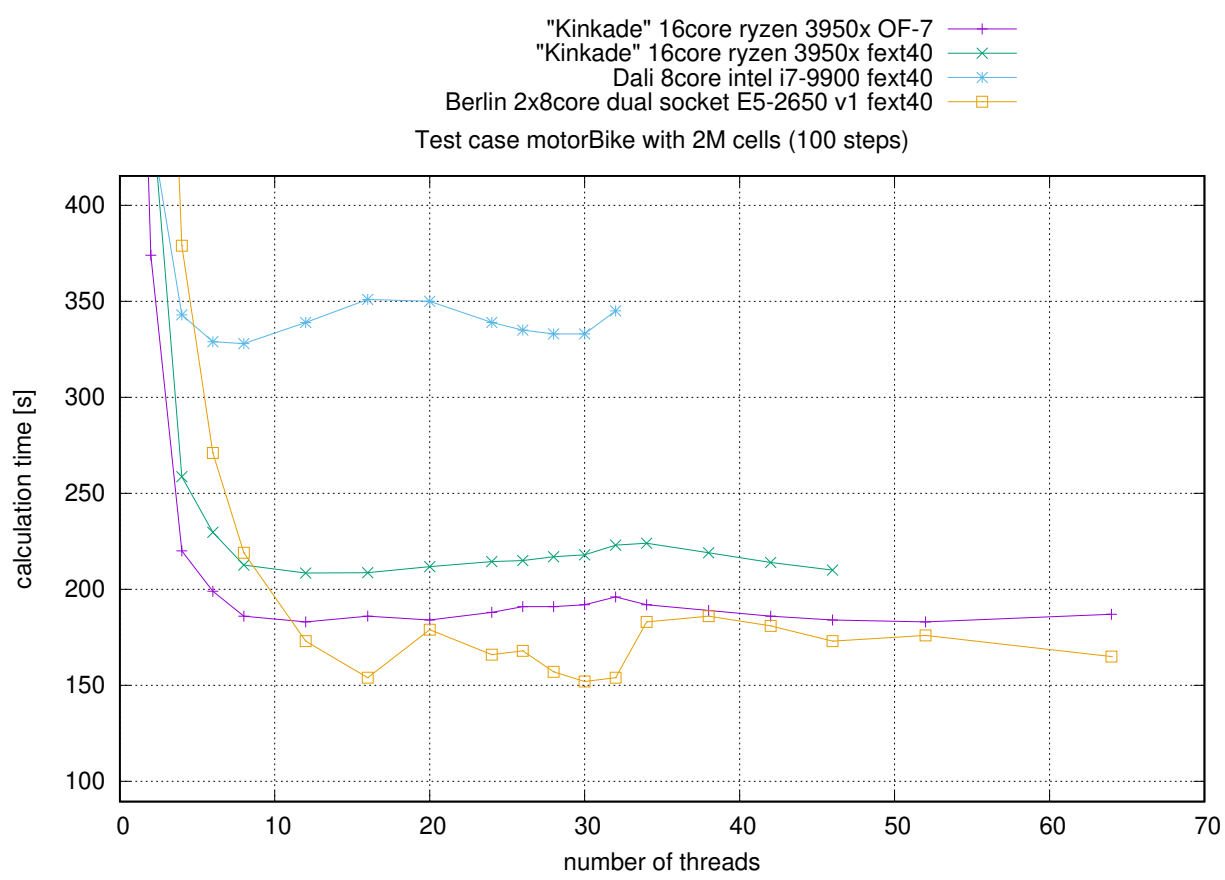

Fig. B.15: Zoom into Fig. B.14.

Scientific Cluster The full 3D Mesh 3.1.2.d mesh was tested on a scientific cluster, too. The

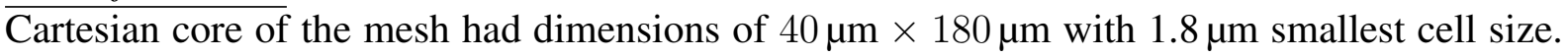
This amounted to $6,407,940$ cells in total. Tab. B.1 shows the results of calculation time comparison to the Xeon-E5 machine.

\begin{tabular}{lrrrrr} 
machine & cores & cells/core & virtual time & execution time & timesteps \\
\hline Cluster & 300 & 21360 & $1.393645005 \mathrm{e}-05$ & $78476.62 \mathrm{~s}$ & 5114 timesteps \\
berlin & 30 & 213600 & $1.376547079 \mathrm{e}-05$ & $119459.32 \mathrm{~s}$ & 5114 timesteps \\
berlin & 30 & 213600 & $1.393914729 \mathrm{e}-05$ & $120100.27 \mathrm{~s}$ & 5141 timesteps \\
\hline speedup: & & & & & \\
same time & 1.53 & & & & \\
same timesteps & & & & &
\end{tabular}

Table B.1: Scientific cluster calculation-time properties.

This reveals a calculation time speedup of 1.52 for a tenfold amount of cores. If the waiting time of the queue of $1 \mathrm{~h} 04 \mathrm{~min} 09 \mathrm{sec}=3849 \mathrm{~s}$ and the time for data withdrawal from the cluster to the home workstation $(6.3 \mathrm{~h}=22680 \mathrm{~s})$ are taken into account, too, then the speedup factor reduces to 1.144 .

In another test case the calculation was spread over 26 nodes and the total speedup resulted into 0.79. Thus it can be deduced that the same conclusion for the bottleneck of CFD holds for a cluster as well.

RapidCFD - Tesla K20M There exists a fork of OpenFOAM which is written in CUDA for graphics cards, called RapidCFD. First of all, the major draw-back of calculating on a GPU - except for the smaller community - is the limited RAM on the graphics card. With $5 \mathrm{~GB}$ RAM only small cases can be set up that can also easily be solved on a normal CPU. For testing 


\section{B Appendix - Numerics}

the Tesla card, the interFoam damBreak tutorial case of OpenFoam 2.3.0 was taken, because the compressibleInterFoam did not compile. The results are shown in Tab. B.2.

\begin{tabular}{lrrr} 
cells & Tesla K20M & 2x Xeon E5-2650 & Core i7-2600 \\
\hline 2000 & $89 \mathrm{~s}$ & - & $(1$ core $) 4 \mathrm{~s}$ \\
$1 \mathrm{M}$ & $558.05 \mathrm{~s}$ & (32 threads) $309.28 \mathrm{~s}$ & (4 threads) $1687.29 \mathrm{~s}$
\end{tabular}

Table B.2: Calculation times of the interFoam damBreak tutorial case, small and large amount of cells, on an Nvidia Tesla K20M and a Xeon i7-2600 machine.

Even though the Tesla GPU has twice as much memory bandwidth as the Xeon-E5 machine it is still slower for the test case with 1000000 cells.

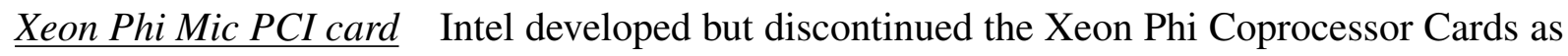
an answer to Nvidia's scientific GPUs. At first it seemed promising, because it was said that the programming language of usual CPUs could be used but the amount of cores was considerably larger. However, it turned out that the coprocessor cards from Intel needed special care for programming as well. Also, setting up the card on an Ubuntu system was not straight forward. Fig. B.16 shows that the Ryzen machine outperforms the Xeon Phi coprocessor easily. Therefore, the effort to set up foam-extend for the Intel coprocessor card does not pay off.

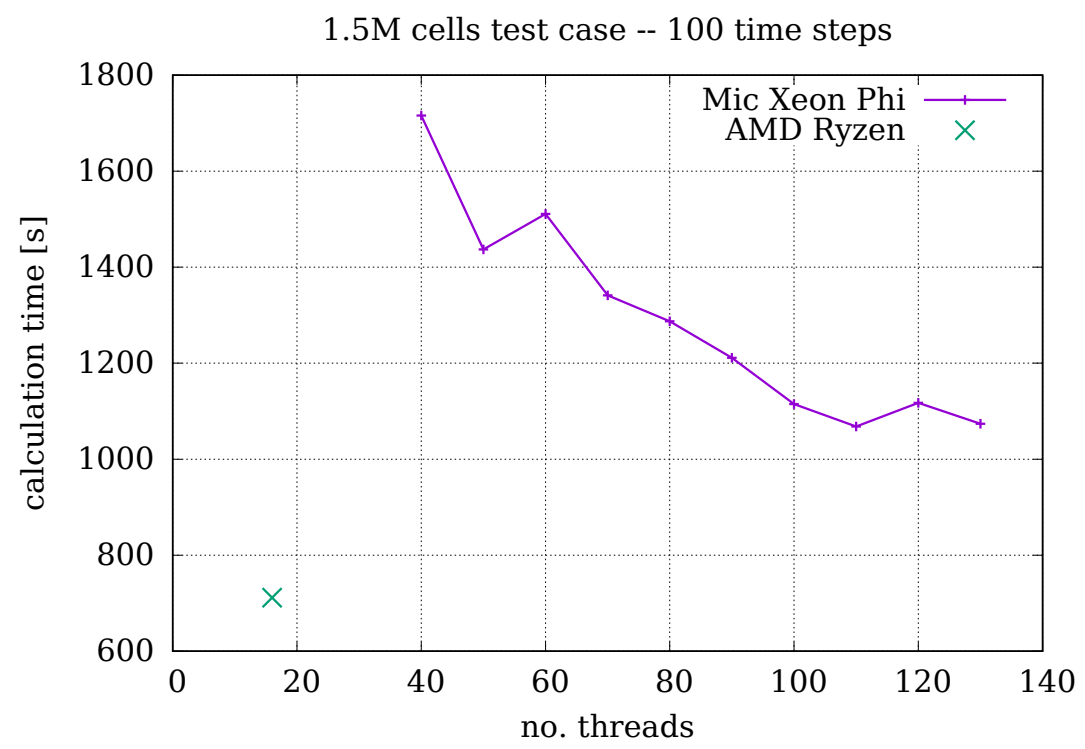

Fig. B.16: Comparison of the calculation times of a test case for the Xeon Phi coprocessor and the Ryzen machine. 


\section{Experiments}

\section{C.1 Illumination}

\section{C.1.1 Overdriven $100 \mathrm{~W}$ LED}

A second, more elaborate version of the device to overdrive a $100 \mathrm{~W}$ LED was tested concerning the light output (Joule per microsecond). It was even a double LED (red) arrangement, but the light yield amounted only to $26.6707 \mu \mathrm{J} / \mu \mathrm{s}$.

The flash pulse duration was set to $1 \mu$ s and was powered with $166 \mathrm{~V}$ (overdriving factor of 6). The triggering was done with 60 pulses of a $100 \mathrm{kHz}$ square signal. For detecting light intensity, the Thorlabs SV2-FC high speed photodiode was used. The following calculation was done without error estimation, just to estimate the magnitude of light yield:

The formula to obtain the incident light power $P$ is given in the data sheet of the photodiode as

$$
P=\frac{V_{\text {out }}}{R(\lambda) \cdot R_{\text {load }}}
$$

where $V_{\text {out }}$ is the voltage produced by the photodiode, $R(\lambda)$ is the spectral responsiveness given in the manual and $R_{\text {load }}$ is the $50 \Omega$ load resistance of the BNC connection. The first trigger pulse of the trigger burst was observed with the oscilloscope. The maximum height of the pulse was measured by the oscilloscope's cursor function. With the red LEDs the waveform of the pulse was found to be the one in Fig. C.1.

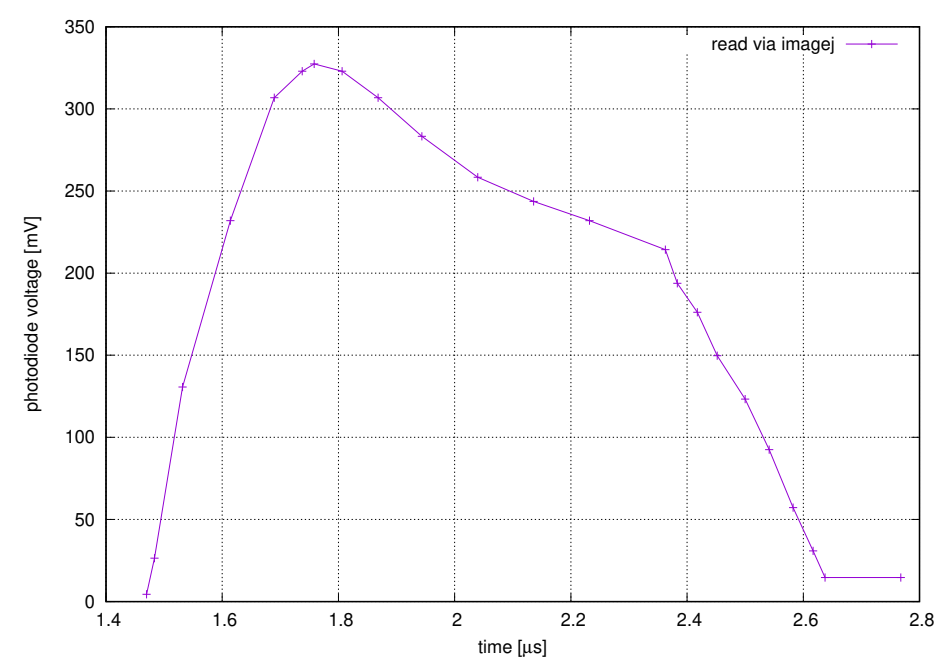

Fig. C.1: Pulse characteristics of the photodiode voltage when using red LEDs.

The setup to measure the intensity was kept simple and run manually. A ruler was attached to the leading of the photodiode as shown in Fig. C.2. 


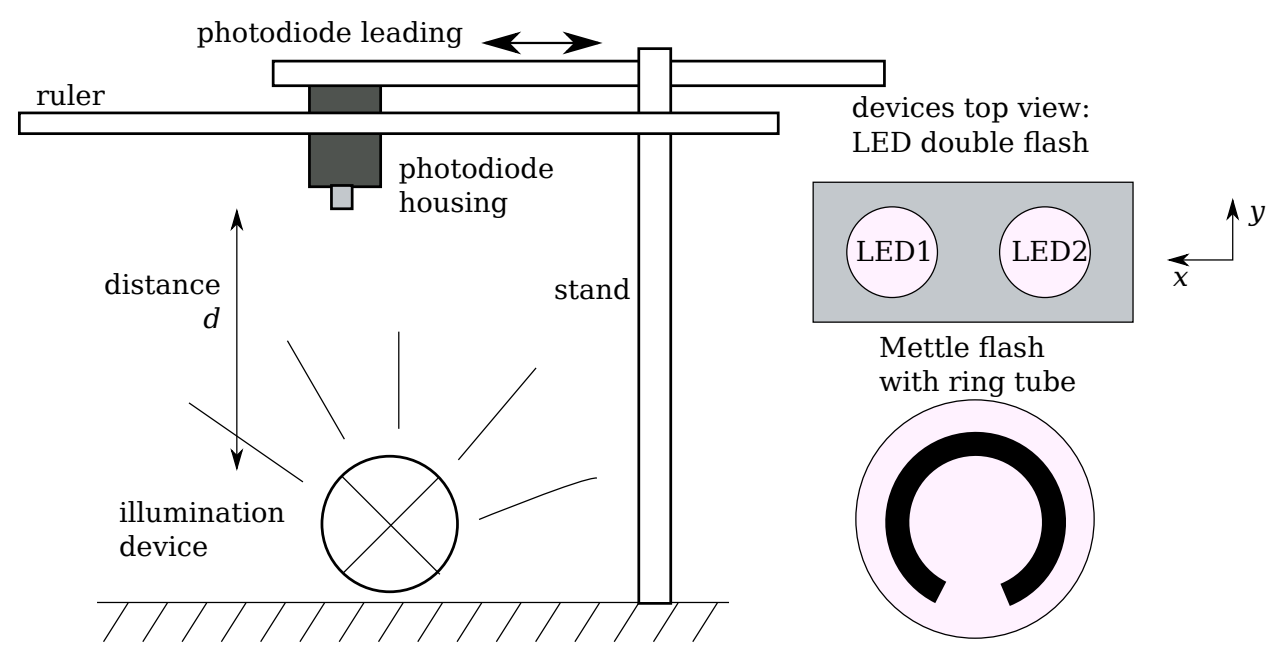

Fig. C.2: Sketch of the setup. The ruler is parallel to the leading and the tip of the photodiode. For the LED the distance of the photodiode tip to the tip of the lenses is set to $8 \mathrm{~cm} \pm 0.2 \mathrm{~cm}$.

The flash was measured along the axis of the LEDs ("x-axis"), as well as orthogonal to it above one of the LEDs ("y-axis").

The measurement values obtained for the two directions are shown in Fig. C.3.

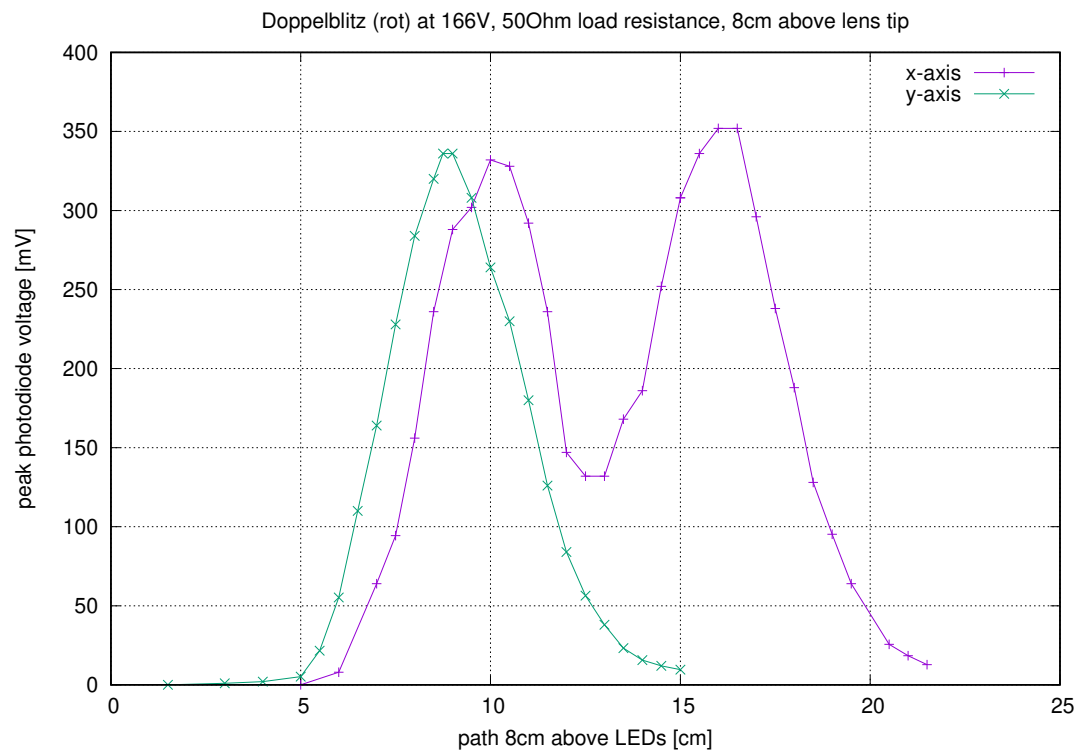

Fig. C.3: Raw measurement values

In order to correlate the voltage of the maximum height of the flash pulse with the emitted energy, first of all the value of $R(\lambda)$ of Equation C.1 had to be determined. For this, the spectrum of the data-sheet was digitalized (see Fig. C.4). A value of $R(\lambda=635 \mathrm{~nm})=0.4$ was taken. 

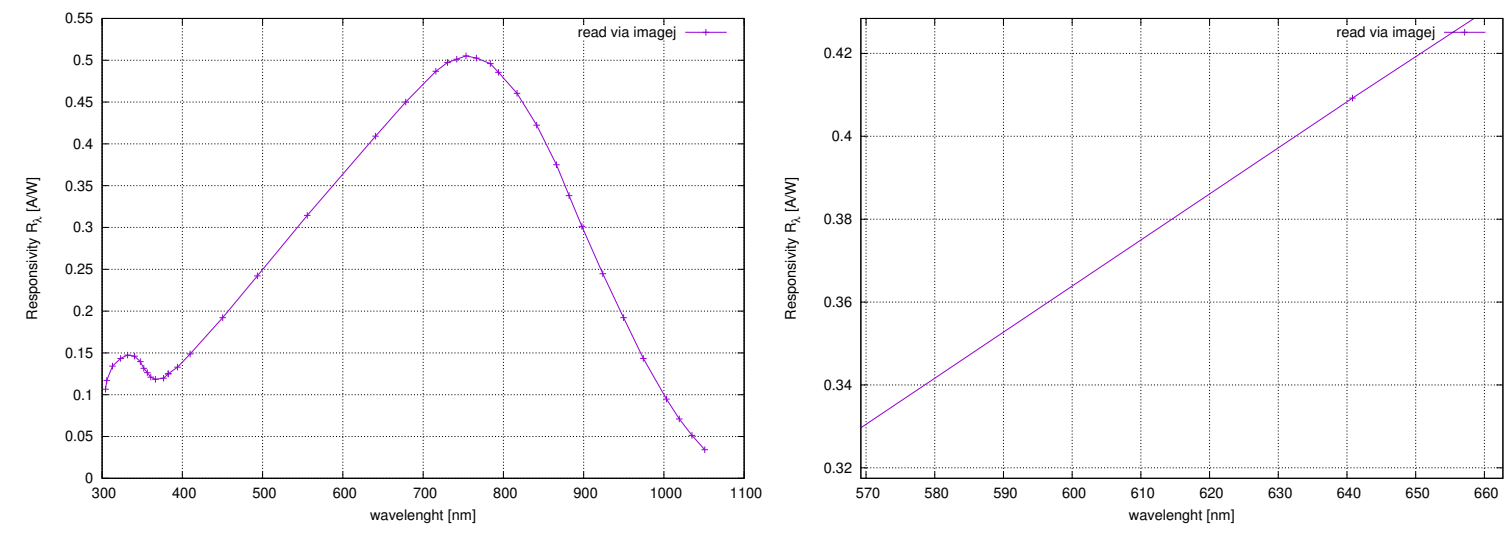

Fig. C.4: Spectrum of the SV2-FC photodiode.

By Eq. C.1 the waveform $U(t)$ of the pulse of Fig. C.1 was then transformed into values of light power per photodiode active area $(\varnothing 4 \mathrm{~mm})$

$$
e=\int P(U(t)) \mathrm{d} t \approx 12.74 \frac{\mathrm{nJ}}{\pi(0.2 \mathrm{~mm})^{2}}
$$

and correlated to the maximum voltage of the waveform (integration of the waveform by midpoint rule)

$$
\text { e } \propto U_{\max } \approx 327.34 \mathrm{mV} .
$$

As a thumb rule, the energy per photodiode active area was assumed to scale with the maximum voltage of the waveform:

$$
c_{\mathrm{U} 2 \mathrm{E}}=\frac{e}{U_{\max }}=30.97 \frac{\mathrm{nJ}}{\mathrm{mV} \mathrm{cm}^{2}}
$$

This conversion factor was used to convert the raw measurement values of Fig. C.3 to energy values. The results including Gaussian fit functions are shown in Fig. C.5.

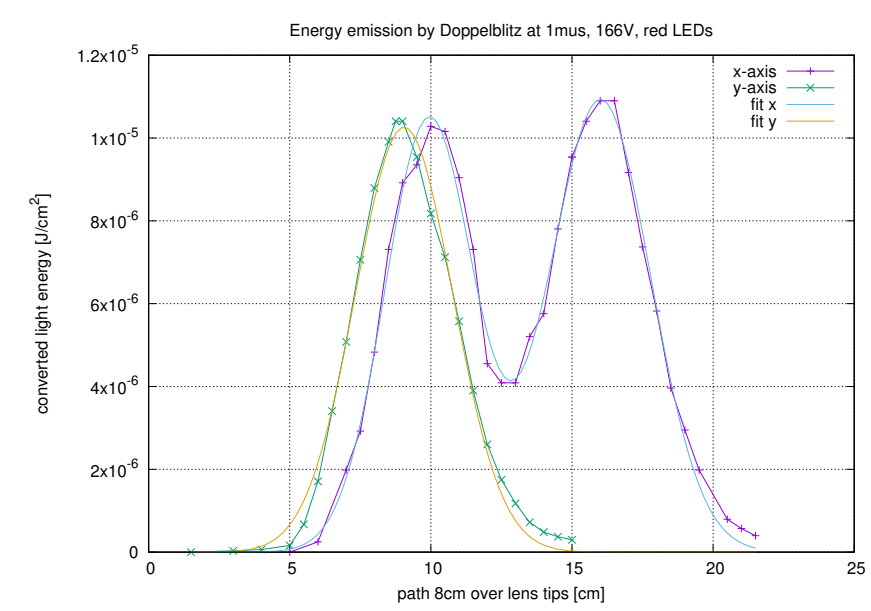

Fig. C.5: Converted measurement values and Gaussian fit functions

The fit functions for the two measurement directions are:

$$
\begin{array}{r}
f(x)=\mathrm{e}^{-\frac{\left(x-x_{s 1}\right)^{2}}{\sigma_{x 1}}} \cdot A_{1}+\mathrm{e}^{-\frac{\left(x-x_{s 2}\right)^{2}}{\sigma_{x 2}}} \cdot A_{2} \\
g(y)=\mathrm{e}^{-\frac{\left(y-y_{s}\right)^{2}}{\sigma_{y}}} \cdot A_{y},
\end{array}
$$




\section{Experiments}

where the $x_{i}$ and the $y_{s}$ are the offsets towards the local intensity maxima, the $\sigma_{i}$ are the squared widths of the Gaussian packages and the $A_{i}$ are the amplitudes. Fit parameters are shown in Table C.1.

\begin{tabular}{|r|l|l|l|} 
& value & error & unit \\
\hline$\sigma_{x 1}$ & 4.88536 & 0.2445 & $\mathrm{~cm}^{2}$ \\
$\sigma_{x 2}$ & 6.36906 & 0.2903 & $\mathrm{~cm}^{2}$ \\
$A_{1}$ & $1.04863 \cdot 10^{-5}$ & $1.879 \cdot 10^{-7}$ & $\mathrm{~J} / \mathrm{cm}^{2}$ \\
$A_{2}$ & $1.09307 \cdot 10^{-5}$ & $1.71 \cdot 10^{-7}$ & $\mathrm{~J} / \mathrm{cm}^{2}$ \\
$x_{s 1}$ & 9.94261 & 0.03587 & $\mathrm{~cm}$ \\
$x_{s 2}$ & 16.0116 & 0.0352 & $\mathrm{~cm}$ \\
$\sigma_{y}$ & 5.97806 & 0.214 & $\mathrm{~cm}^{2}$ \\
$A_{y}$ & $1.0263 \cdot 10^{-5}$ & $1.471 \cdot 10^{-7}$ & $\mathrm{~J} / \mathrm{cm}^{2}$ \\
$y_{s}$ & 9.05388 & 0.03153 & $\mathrm{~cm}$
\end{tabular}

Table C.1: Fit parameters for Gaussian fits.

With these very good fits, a two-dimensional intensity function $I(x, y)$ was created from Eqs. C.3,C.4 to extrapolate to the whole field of flash emission (with $y_{s}=0, A_{y}=0$ ):

$$
I(x, y)=\left(\mathrm{e}^{-\frac{\left(x-x_{s 1}\right)^{2}}{\sigma_{x 1}}} \cdot A_{1}+\mathrm{e}^{-\frac{\left(x-x_{s 2}\right)^{2}}{\sigma_{x 2}}} \cdot A_{2}\right) \cdot \mathrm{e}^{-\frac{y^{2}}{\sigma_{y}}}
$$

The plot is shown in Fig. C.6.

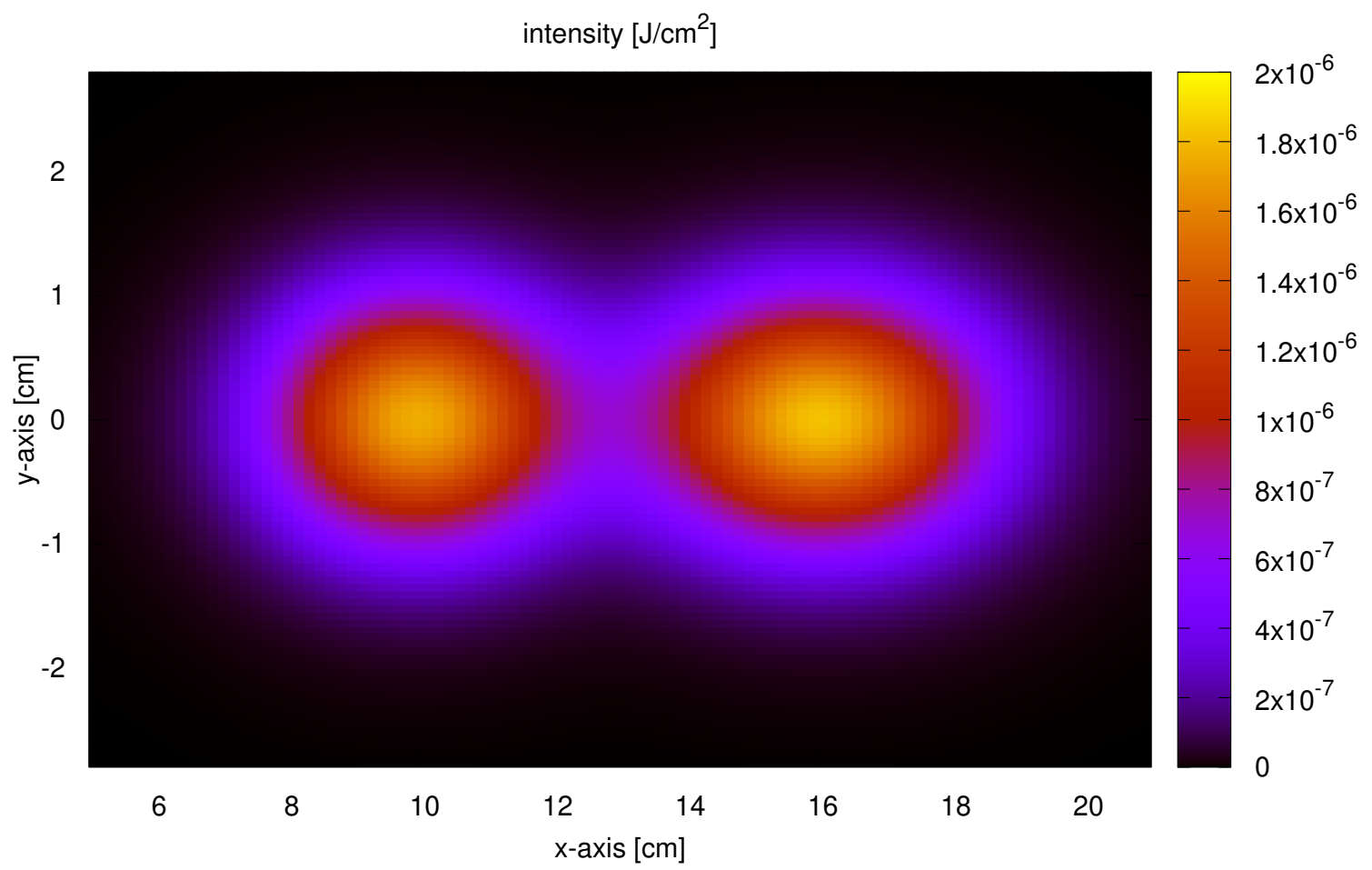

Fig. C.6: Plot of the fit function $I(x, y)$ of Eq. C.5. 


\section{Experiments}

In order to finally receive the Joule output, Eq. C.5 was numerically integrated. The python script is given in the repository B.3 in the "exp" folder. It was run with

python integrate.py -a 4 -b 22 -c "-3" -d 3 -s 100 -e 10000 -t 1e-5

To find the final result:

$$
I_{1 \mu \mathrm{s}, 166 \mathrm{~V}}=26.67 \mu \mathrm{J}
$$


C Experiments 


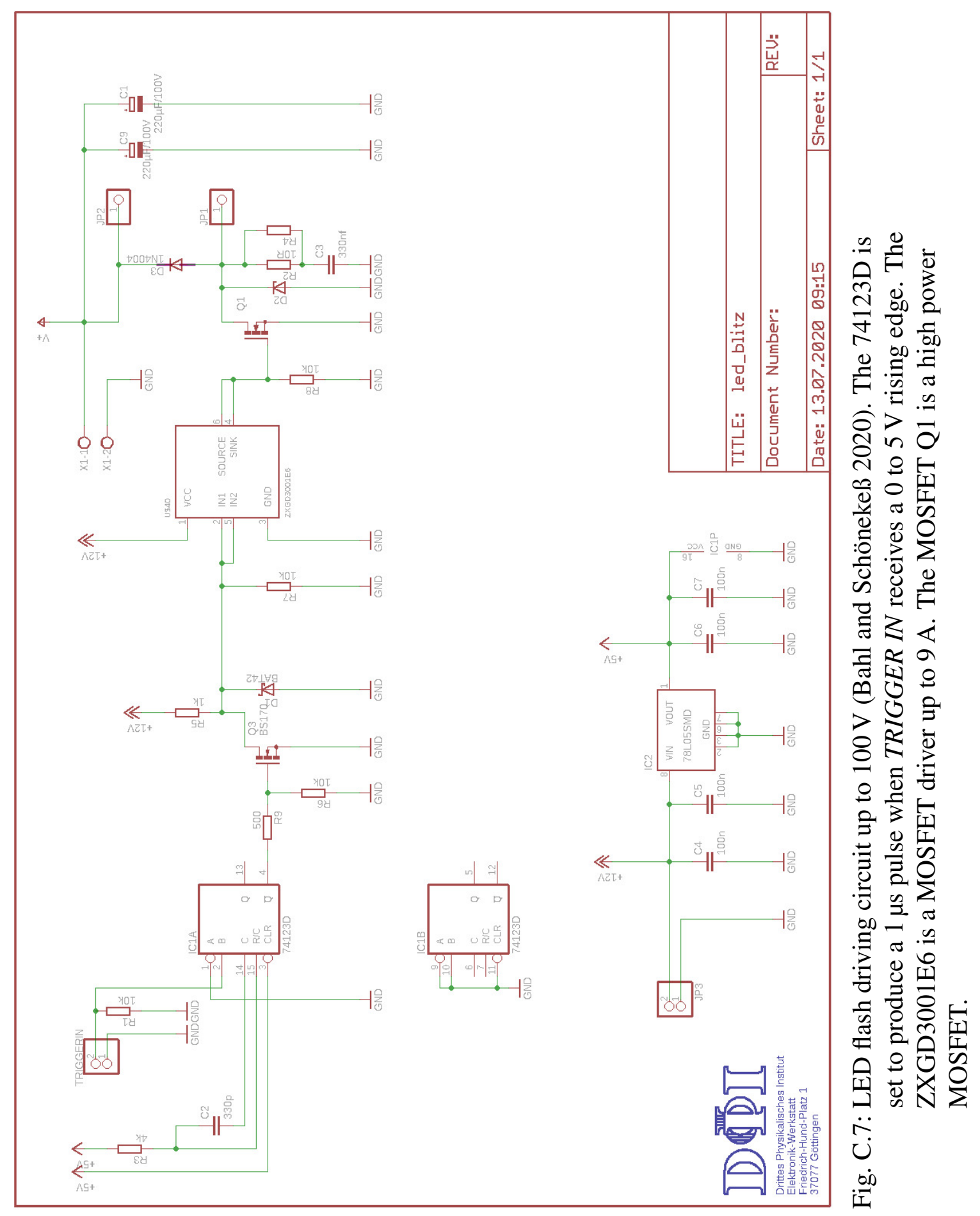




\section{C.1.2 METTLE flash}

The energy output (Joule per microsecond) of the $600 \mathrm{Ws}$ METTLE flash is measured. For detecting light intensity, the Thorlabs SV2-FC high speed photodiode was used. The light yield amounts to $31.55 \mathrm{~mJ}$ per $1 \mu \mathrm{s}$. This is a factor of 1182 higher than the 6 times overdriven LED.

The formula to obtain the incident light power $P$ for the photodiode is stated in Eq. C.1 Because the METTLE flash emits a spectrum, the formula has to be written as

$$
V_{\text {out }}=R_{\text {load }} \cdot \mathrm{P} \cdot \int P(\lambda) R(\lambda) \mathrm{d} \lambda
$$

where

$$
\int P(\lambda) \mathrm{d} \lambda=1, \quad \text { such that } \int \mathrm{P} P(\lambda) \mathrm{d} \lambda=\mathrm{P} .
$$

A normalized black body spectrum of daylight temperature $(5777 \mathrm{~K})$ was assumed for $P(\lambda)$ of the METTLE flash lamp:

$$
\begin{array}{r}
s(\lambda)=\frac{2 \pi h c^{2}}{\lambda^{5}} \frac{1}{\mathrm{e}^{\frac{h c}{\lambda k T}}-1}, \quad \int_{305 \mathrm{~nm}}^{1050 \mathrm{~nm}} s(\lambda) \mathrm{d} \lambda=N \\
P(\lambda)=s(\lambda) / N
\end{array}
$$

The flash was set to highest power ("6.0"). It was triggered via a TTL signal that made a FET-transistor close circuit the two poles of the "sync" connection. The maximum voltage of the photodiode pulse was measured by the oscilloscope's cursor function. The waveform of the pulse was found to be the one in Fig. C.8.

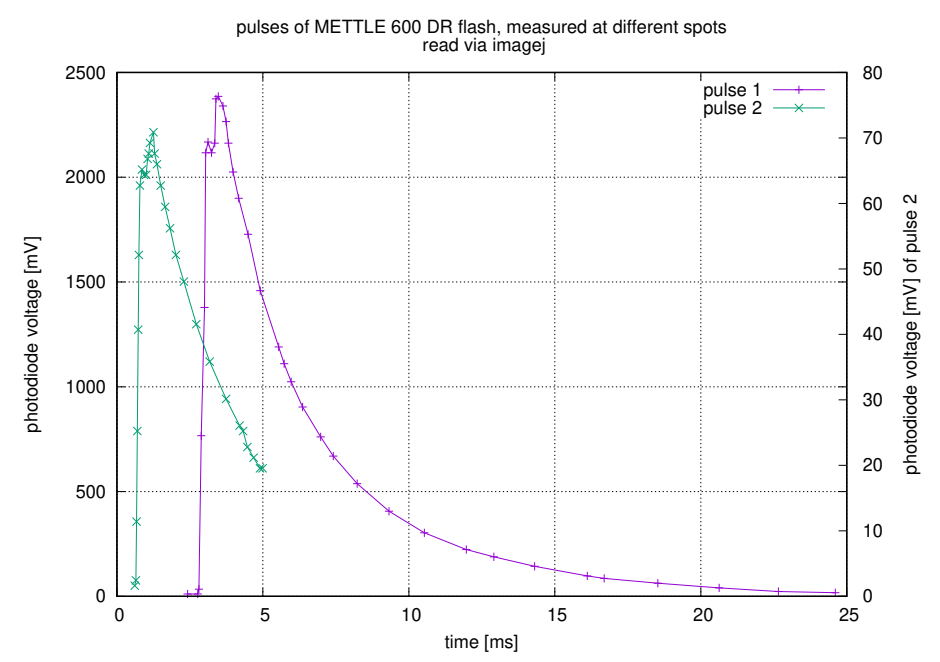

Fig. C.8: Pulse characteristics of the photodiode voltage when using METTLE flash at highest power.

The setup was kept as simple as in Fig. C.2 and run manually. The light emission was measured along the diameter of the reflector shield, approximately $8.5 \mathrm{~cm} \pm 0.3 \mathrm{~cm}$ above the reflector rim.

The measurement values obtained are shown in Fig. C.9. 


\section{Experiments}

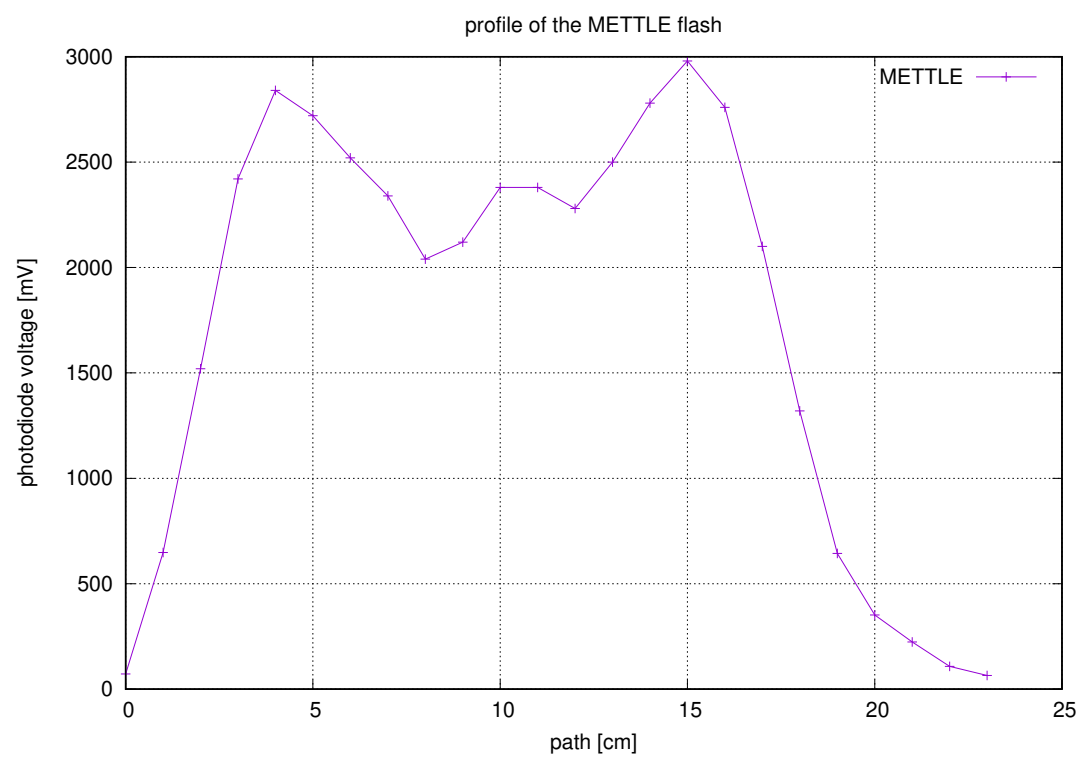

Fig. C.9: Raw measurement values

In order to correlate the voltage of the maximum height of the flash pulse with the emitted energy, first of all the value of the integral of Eq. C.6 had to be determined. For this, the spectrum $R(\lambda)$ of the data-sheet of the photodiode was multiplied with $P(\lambda)$ of Eq. C.7. The result is shown in Fig. C.10. The integral values to

$$
\int P(\lambda) R(\lambda) \mathrm{d} \lambda=0.29956,
$$

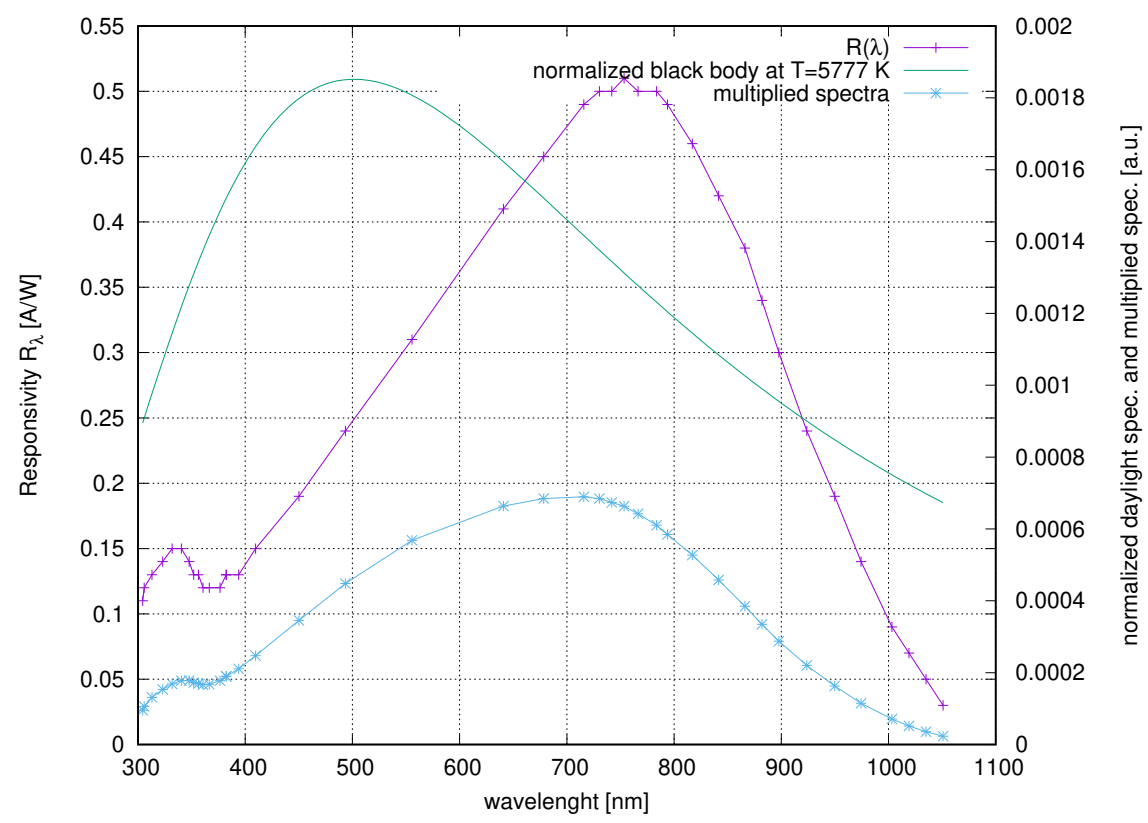

Fig. C.10: Spectra of the SV2-FC photodiode, the normalized daylight black body radiation and the two multiplied together.

which is slightly lower than the value for the red LEDs. With this value and Eq. C.1 the waveforms $U_{i}(t)$ of the pulses of Fig. C.8 were then transformed into values of light power per 


\section{Experiments}

photodiode active area $(\varnothing 0.4 \mathrm{~mm})$

$$
\begin{aligned}
& e_{1}=\int P\left(U_{1}(t)\right) \mathrm{d} t \approx 621.248 \frac{\mathrm{nJ}}{\pi(0.2 \mathrm{~mm})^{2}} \\
& e_{2}=\int P\left(U_{2}(t)\right) \mathrm{d} t \approx 12.1209 \frac{\mathrm{nJ}}{\pi(0.2 \mathrm{~mm})^{2}} .
\end{aligned}
$$

Those energies were correlated to the maximum voltage of the respective waveform (integration of the waveform by midpoint rule)

$$
\begin{aligned}
e_{1} \quad \leftrightarrow \quad U_{1, \max }=2385.67 \mathrm{mV} \quad \Rightarrow \frac{e_{1}}{U_{1, \max } \pi(0.2 \mathrm{~mm})^{2}}=2.07227 \cdot 10^{-4} \frac{\mathrm{J}}{\mathrm{mV} \mathrm{cm}^{2}} \\
e_{2} \quad \leftrightarrow \quad U_{2, \max }=70.88 \mathrm{mV} \quad \Rightarrow \frac{e_{2}}{U_{2, \max } \pi(0.2 \mathrm{~mm})^{2}}=1.36091 \cdot 10^{-4} \frac{\mathrm{J}}{\mathrm{mV} \mathrm{cm}}
\end{aligned}
$$

The second factor of value 1.36091 seems to be reasonably lower because the second pulse was not recorded till the end (Fig. C.8 - green line). That is why the first conversion factor of $2.07227 \cdot 10^{-4} \frac{\mathrm{J}}{\mathrm{mV} \mathrm{cm}^{2}}$ was taken.

This conversion factor was used to convert the raw measurement values of Fig. C.9 to energy values. The results including a triple Gaussian fit function are shown in Fig. C.11.

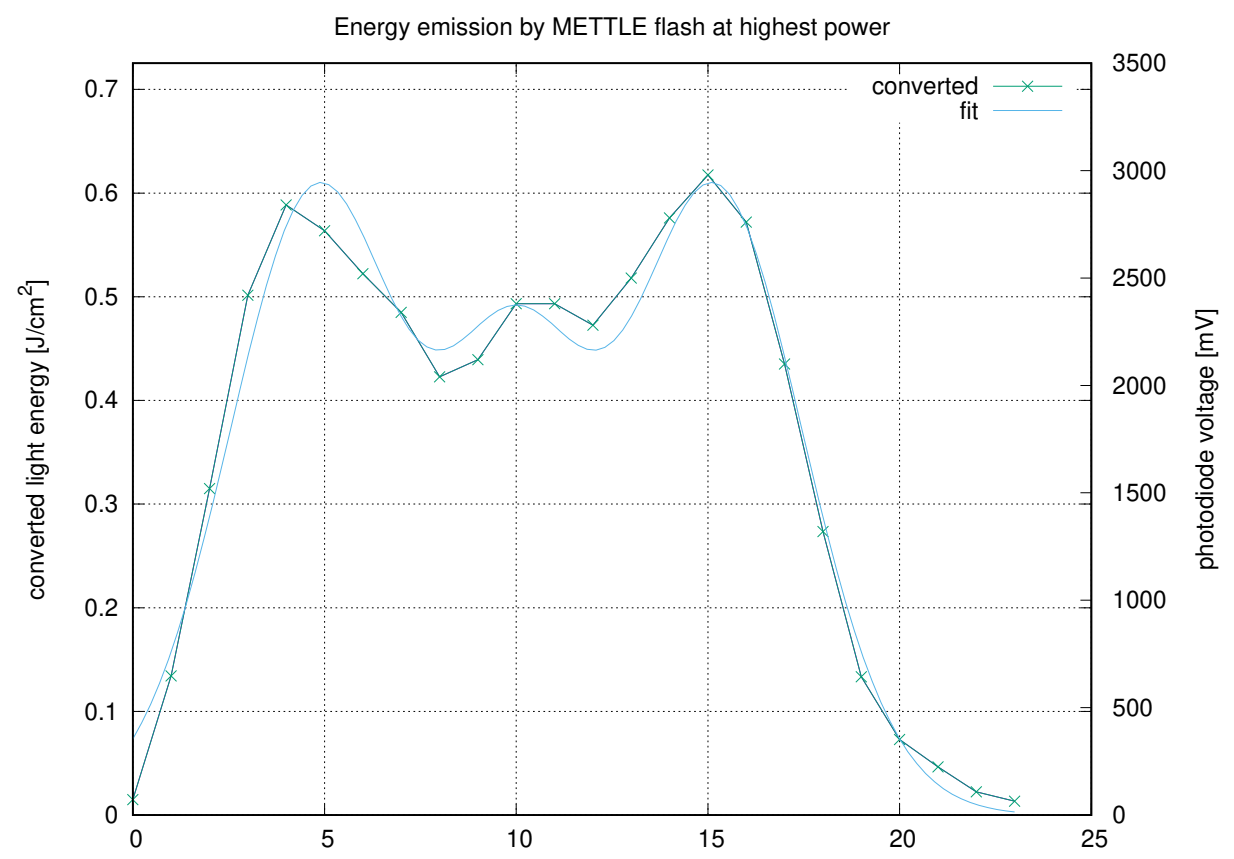

Fig. C.11: Converted measurement values and Gaussian fit functions

The fit function is:

$$
f(x)=\mathrm{e}^{-\frac{(x-10 \mathrm{~cm})^{2}}{\sigma_{x 1}^{2}}} \cdot A_{1}+\mathrm{e}^{-\frac{(x-10 \mathrm{~cm})^{2}}{\sigma_{x 2}^{2}}} \cdot A_{2}+\mathrm{e}^{-\frac{(x-10 \mathrm{~cm})^{2}}{\sigma_{x 3}^{2}}} \cdot A_{3}
$$

where the offset of all terms is fixed to $10 \mathrm{~cm}$, the $\sigma_{i}$ are the widths of the Gaussian packages and the $A_{i}$ are the amplitudes. Fit parameters are shown in Table C.2. 


\begin{tabular}{|c|l|l|} 
& value & unit \\
\hline$\sigma_{x 1}$ & 4.30323 & $\mathrm{~cm}$ \\
$\sigma_{x 2}$ & 4.02335 & $\mathrm{~cm}$ \\
$\sigma_{x 3}$ & 3.67499 & $\mathrm{~cm}$ \\
$A_{1}$ & 47.4406 & $\mathrm{~J} / \mathrm{cm}^{2}$ \\
$A_{2}$ & -76.5306 & $\mathrm{~J} / \mathrm{cm}^{2}$ \\
$A_{3}$ & 29.5822 & $\mathrm{~J} / \mathrm{cm}^{2}$
\end{tabular}

Table C.2: Fit parameters for Gaussian fits.

Assuming rotational symmetry, a two-dimensional intensity function $I(x, y)$ was created from Eq. C.9 to extrapolate to the whole field of flash emission:

$$
I(x, y)=\sum_{i=1}^{3} \mathrm{e}^{-\frac{x^{2}+y^{2}}{\sigma_{x, i}^{2}}} \cdot A_{i}
$$

The plot is shown in Fig. C.12.

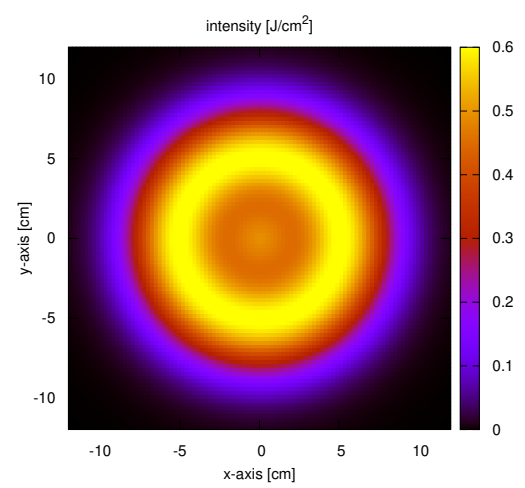

Fig. C.12: Plot of the fit function $I(x, y)$ of Eq. C.10.

In order to finally receive the Joule output, Eq. C.10 was numerically integrated. The python script is found again in the repository B.3. It was run with

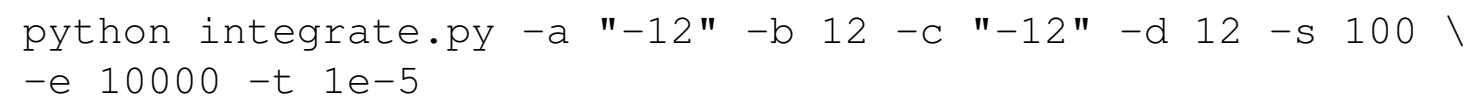

to find the final result:

$$
I_{M E T T L E}=122.86 \mathbf{J}
$$

In order to compare the Energy output of the METTLE-Blitz to the LED DOPPEL-Blitz, a $1 \mu$ s window was taken out of each pulse signal where the amplitude is highest. The maximum of the pulse lasts about $78 \mu \mathrm{s}$, so we can assume power stability over the $1 \mu \mathrm{s}$ window. This leads to a conversion factor of

$$
\begin{aligned}
& e_{3}=1.59279 \cdot 10^{-7} \frac{\mathrm{J}}{\pi(0.2 \mathrm{~mm})^{2}} \leftrightarrow \quad U_{3, \max }=2385,67 \mathrm{mV} \\
& \Rightarrow \frac{e_{3}}{U_{3, \max } \pi(0.2 \mathrm{~mm})^{2}}=5.31297 \cdot 10^{-8} \frac{\mathrm{J}}{\mathrm{mV} \mathrm{cm}^{2}} .
\end{aligned}
$$


The new fit parameters are shown in Tab. C.3. The final result is:

$$
I_{M E T T L E, 1 \mu \mathrm{s}}=31.55 \mathrm{~mJ}
$$

which is still by a factor of 1,000 higher than the Energy from the LEDs.

\begin{tabular}{|c|l|l|} 
& value & unit \\
\hline$\sigma_{x 1}$ & 4.09533 & $\mathrm{~cm}$ \\
$\sigma_{x 2}$ & 4.07853 & $\mathrm{~cm}$ \\
$\sigma_{x 3}$ & 3.83306 & $\mathrm{~cm}$ \\
$A_{1}$ & 0.447996 & $\mathrm{~J} / \mathrm{cm}^{2}$ \\
$A_{2}$ & -0.475446 & $\mathrm{~J} / \mathrm{cm}^{2}$ \\
$A_{3}$ & 0.0275761 & $\mathrm{~J} / \mathrm{cm}^{2}$
\end{tabular}

Table C.3: Fit parameters for the $1 \mu$ s Gaussian fits. 


\section{Acknowledgements}

Here is the space for honesty.

I deeply thank Dr. Robert Mettin and Prof. Dr. Werner Lauterborn for their motivating guidance in the work with bubbles all those years. The humour, exceptional knowledge and pressure-less leadership of Robert Mettin made me feel home and at the same time very productive. I thank Werner Lauterborn for his restless support, motivation and universe of experience that motivated even in paper rejection times.

A heartfelt thanks goes to the bubble guys and girls: Dr. Christiane Lechner, the one who solves any equation and algorithm problem even if unsolvable and whom I may call colleague, friend and supervisor at the same time, Juan M. Rosselló (called J or Dr. Nobody), Ferenc Hegedús, Julian Eisener who went to the battlefields of lab moving with me, Hendrik Söhnholz, Dwayne Stephens and Ekim Büsra Sarac, Fabian Reuter, Julia Schneider, Matti Tervo, Bernhard Lindinger, Roxana Vargas, the secretaries Sabine Huhnold and Elke Zech, the IT support Thomas Geiling, the workshop guys Dieter Hille and Team, Markus Schönekeß and Team and Simon Bahl and Prof. Dr. Ulrich Parlitz for his year long loyalty to the DPI-Team. Altogether the best team, ever.

Special thanks go to Claus-Dieter Ohl, who made it possible that I may have worked on a workstation in the home office during Corona time.

Enormous thanks go to my father Andreas Koch, mother Ursula Koch and brother Henry Koch for their yearlong support and endurance.

I deeply, gratefully thank my wife Sunny Ursa Liebscher-Koch for her love and energy support. Without her patience and care for the strangeness of the ideas of academic minds, I would still not touch the ground.

\section{Official acknowledgements}

The funding for this project by the Deutsche Forschungsgemeinschaft (German Research Foundation) under contract Me 1645/8-1 is gratefully acknowledged. 


\section{Affidavit}

I hereby confirm that my thesis entitled Laser cavitation bubbles at objects: Merging numerical and experimental methods is the result of my own work. I did not receive any help or support from commercial consultants. All sources and / or materials applied are specified in the thesis.

Furthermore, I confirm that this thesis has not yet been submitted as part of another examination process neither in identical nor in similar form.

Göttingen, 21. Nov. 2020 